PNL-9450-1

UC-721

\title{
INTERNATIONAL NUCLEAR \\ WASTE MANAGEMENT FACT BOOK
}

I. W. Leigh

May 1994

Prepared for

the U.S. Department of Energy

Office of Environmental Management

Waste Management Office

under Contract DE-AC06-76RLO 1830

Pacific Northwest Laboratory

Richland, Washington 99352 


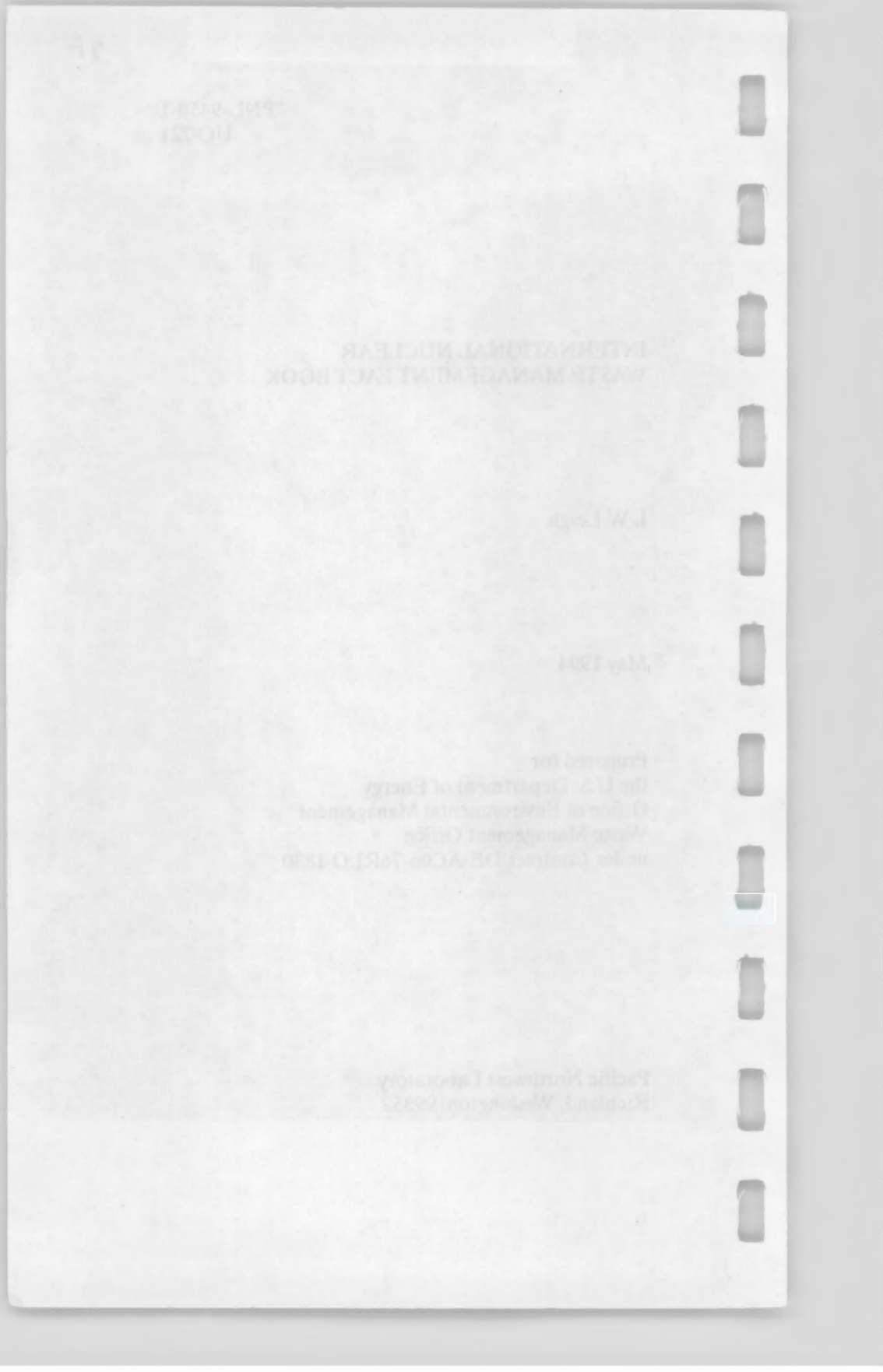




\title{
PREFACE
}

As the U.S. Department of Energy (DOE) and DOE contractors have become increasingly involved with other nations in nuclear fuel cycle and waste management cooperative activities, a need exists for a ready source of information concerning foreign nnclear waste management programs, facilities, and personnel. This Fact Book has been compiled to meet that need.

The information contained in the International Nuclear Waste Management Fact Book has been obtained from many unclassified sources: nuclear trade journals and newsletters; reports of foreign visits and visitors; CEC, IAEA, and OECD/NEA activities reports; and proceedings of conferences and workshops. The data listed typically do not reflect any single source but frequently represent a consolidation/combination of information.

The organizations and agencies listed in this publication often have a much wider range of activities and many more facilities or staff than are described here. Lack of space and the intent of the Fact Book limit the listed information to data primarily pertaining to the nuclear waste management area.

Every effort was made for all information to be as accurate and current as possible, incorporating updates as they became available until actual time of printing; however, the nature of the contents makes it subject to frequent changes. If you have suggestions that would improve the usefulness of the book or if you can provide more current information, please let us know so these changes can be included in future editions.

\author{
International Program Support Office \\ Pacific Northwest Laboratory \\ P.O. Box 999 \\ Richland, WA 99352 \\ Tel: 509-375-6507 \\ Fax: 509-375-6417
}




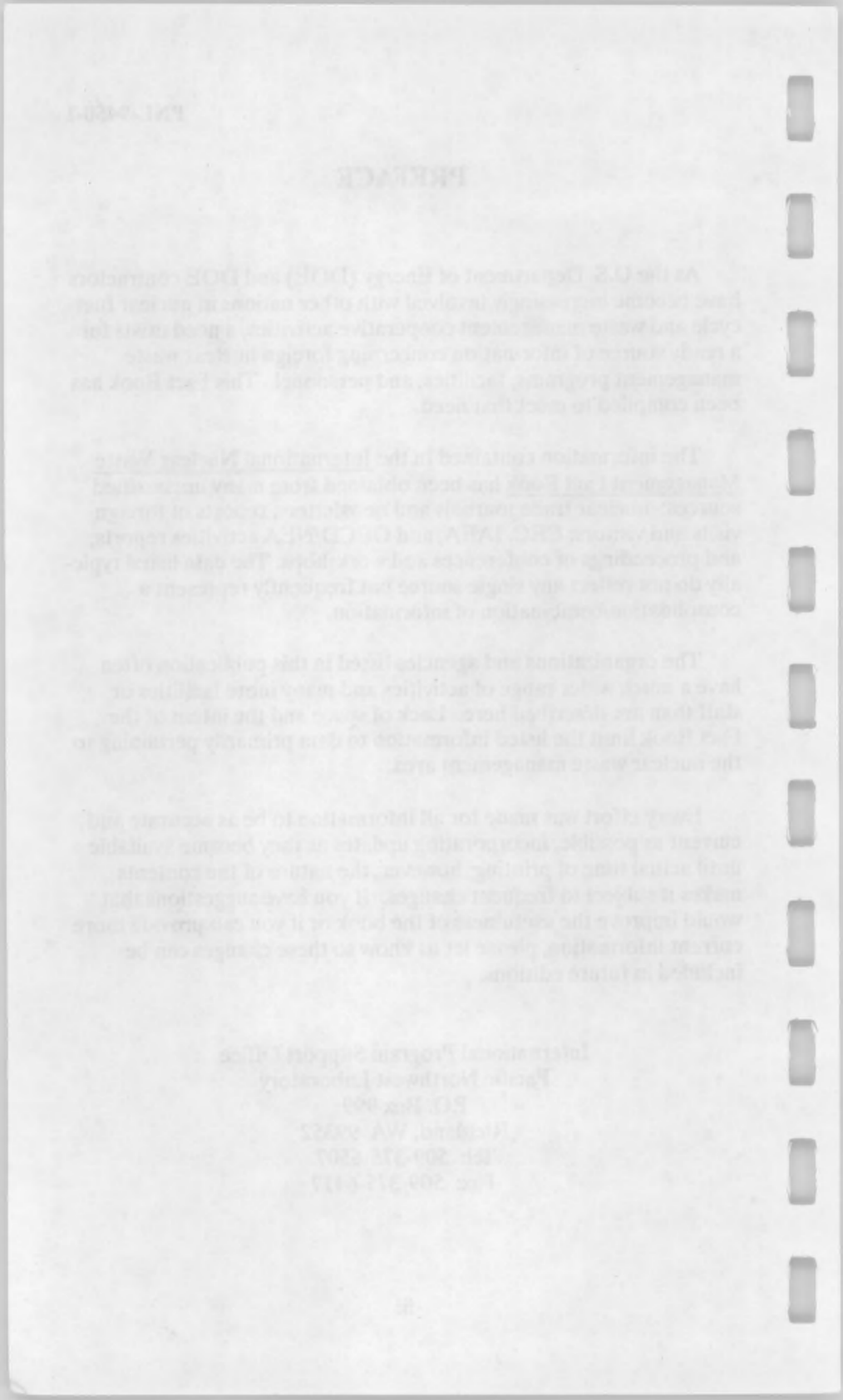




\section{CONTENTS}

Page

Preface $, \ldots \ldots \ldots \ldots \ldots \ldots \ldots \ldots \ldots \ldots, \ldots \ldots, \quad$ ii

Introduction $\ldots \ldots \ldots \ldots \ldots \ldots \ldots \ldots \ldots \ldots \ldots \ldots$ INTRO-1

National Summaries

Aggentina $\ldots \ldots \ldots \ldots \ldots \ldots \ldots \ldots \ldots \ldots \ldots \ldots \ldots \ldots \ldots \ldots$, AR-1

Australia...$\ldots \ldots \ldots \ldots \ldots \ldots \ldots \ldots \ldots \ldots$ AS -1

Belarus $\ldots \ldots \ldots \ldots \ldots \ldots \ldots \ldots \ldots \ldots \ldots \ldots$, BO-1

Belgium $\ldots \ldots \ldots \ldots \ldots \ldots \ldots \ldots \ldots \ldots \ldots \ldots, \quad$ BE-1

Brazil $\ldots \ldots \ldots \ldots \ldots \ldots \ldots \ldots \ldots \ldots \ldots \ldots \ldots \ldots \ldots \ldots \ldots, \quad$ BR-1

Canada ................................. CA-1

China (Peuple's Republic of China) $\ldots \ldots \ldots \ldots \ldots \ldots$ CH-1

Finland $, \ldots \ldots, \ldots \ldots \ldots, \ldots, \ldots \ldots \ldots \ldots \ldots, \quad$ FI-1

France $\ldots \ldots \ldots \ldots \ldots \ldots \ldots \ldots \ldots \ldots \ldots \ldots \ldots, \quad$ FR-1

Germany $\ldots \ldots \ldots \ldots \ldots \ldots \ldots \ldots \ldots \ldots \ldots \ldots$, GE-1

India $, \ldots \ldots \ldots \ldots \ldots \ldots \ldots \ldots \ldots \ldots, \quad$ IN-1

Italy $\ldots \ldots \ldots \ldots \ldots \ldots \ldots \ldots \ldots \ldots \ldots \ldots, \quad \boldsymbol{I} \mathbf{T}-1$

Japan $\ldots \ldots \ldots \ldots \ldots \ldots \ldots \ldots \ldots \ldots \ldots \ldots \ldots, \quad$ JA-1

Korea (Republic of Korea) $\ldots \ldots \ldots \ldots \ldots \ldots \ldots \ldots, \quad$ KS-1

Netherlands $\ldots \ldots \ldots \ldots \ldots \ldots \ldots \ldots \ldots \ldots \ldots \ldots, \quad$ NL-1

Pakistan $\ldots \ldots \ldots \ldots \ldots \ldots \ldots \ldots \ldots \ldots \ldots \ldots, \quad$ PK-1 


\section{PNL-9450-1}

Russia $\ldots \ldots \ldots \ldots \ldots \ldots \ldots \ldots \ldots \ldots \ldots \ldots, \quad$ RS-1

South Africa $\ldots \ldots \ldots \ldots \ldots \ldots \ldots \ldots \ldots \ldots, \quad$ SF-1

Spain $\ldots \ldots \ldots \ldots \ldots \ldots \ldots \ldots \ldots \ldots \ldots \ldots \ldots \ldots \ldots, \quad$ SP-1

Sweden $\ldots \ldots \ldots \ldots \ldots \ldots \ldots \ldots \ldots \ldots \ldots \ldots, \quad$ sw-1

Switzerland $\ldots \ldots \ldots \ldots \ldots \ldots \ldots \ldots \ldots \ldots \ldots, \quad$ SZ-1

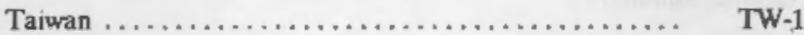

United Kingdom $\ldots \ldots \ldots \ldots \ldots \ldots \ldots \ldots \ldots \ldots \ldots$ UK-1

United States $\ldots \ldots \ldots \ldots \ldots \ldots \ldots \ldots \ldots \ldots \ldots \ldots$ US 1

International Agencies

CEC (Commission of the European Communities) ..... INTL-1

LAEA (International Atomic Energy Agency) ....... INTL-4

ICRP (International Commission on

Radiological Protection) $\ldots \ldots \ldots \ldots \ldots \ldots \ldots \ldots \ldots$ INTL-8

OECD Nuclear Energy Agency (NEA) $\ldots \ldots \ldots \ldots \ldots$ INTL-8

Nuclear Societies $\ldots \ldots \ldots \ldots \ldots \ldots \ldots \ldots \ldots$ INTL -13

Glossary - Organizations, Facilities $\ldots \ldots \ldots \ldots \ldots \ldots \ldots$ G-1

Technical and Other Terms $\ldots \ldots \ldots \ldots \ldots \ldots \ldots, \quad$ G-17 
INTRODUCTION

$\square$

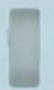

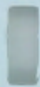

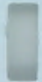

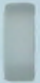

品

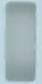

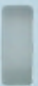

प

口 


\section{INTRODUCTION}

The International Nuclear Waste Management Fact Book has been compiled in an effort to provide current data concerning fuel cycle and waste management facilities, R\&D programs, and key personnel in 24 countries, including the U.S., four multinational agencies, and 21 nuclear societies. This publication succeeds the previously issued International Nuclear Fuel Cycle Fact Book (PNL3594 ), which appeared annually for 13 years. While the title is different, there are no substantial changes in the content.

The Fact Book is organized as follows:

- Nalional summaries--a section for each country that summarizes nuclear policy, describes organizational relationships, and provides addresses and names of key personnel and information on facilities.

- International agencies--a section for each of the international agencies that has significant fuel cycle irvolvement and a listing of nuclear societies.

- Gloseary--a list of abbreviations/actonyms of organizations, facilities, and technical and other terms.

The national summaries, in addition to the data described above, feature a small map for each country as well as some general information. The latter is presented from the perspective of the Fact Book user in the United States. Please note the following: 


\section{DIRECT DIALING}

For convenience in direct dialing from the U.S. to foreign countries, complete telephone numbers are listed, including country and city codes. Outside the U.S., depending on the origin and destination of the call, some of these codes may not be necessary. Instead, " 0 " may need to precede the local number. Because it is impossible to cover the various situations for calls originating outside the U.S., accurate information concerning direct dial is best obtained from local sources (telephone company or hotel operator).

\section{HOLIDAYS}

The major holidays have been listed as they generally apply to the entire country. Regionai holidays are not included, although they may, no doubt, also be considered major in a particular area.

\section{MAPS}

Most of the major facility locations are shown on each country's map within a circle for easier identification. Where space permitted, the name of the organization or facility has been added. The major cities are circied, and some of the smaller towns are listed to assist as a reference when consulting a large-scale map.

\section{PASSPORT/VISA}

Requirements listed are those applicable to U.S. citizens.

\section{SOURCES}

Electric power capacity and production figures given for some countries were obtained from Energy Balances of OECD Countries 1991-1992; OECD/International Energy Agency, Paris, 1994, and Nuclear Energy Data, OECD/Nuclear Energy Agency, Paris, 1993.

Reactor Mix figures were obtained from "World List of Nuclear Power Plants as of 6/93," Nuclear New5, $9 / 93$. 


\section{TIME}

The hours listed indicate the standard time difference between the country and Washington, D.C. A specific reference area/town is identified if more than one time zone exists in a given country. Please note: the stated time difference may be affected by applicable daylight savings time. Also, in some countries (where the seasons are reversed), the standard time period is listed instead of the time during which daylight savings time is in effect.

\section{VISITS TO U.S. DOE FACILITIES}

Foreign visitors to U.S. DOE facilities must complete and submit a form IA-473 (OMB 1910-2100), "Request for Foreign National Unclassified Visit or Assignment," to the DOE facility they wish to visit at least 45 days before the proposed visit. The itinerary should be based on prior arrangement with appropriate DOE or DOE contractor staff concerning a suitable time for the visit. 


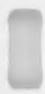

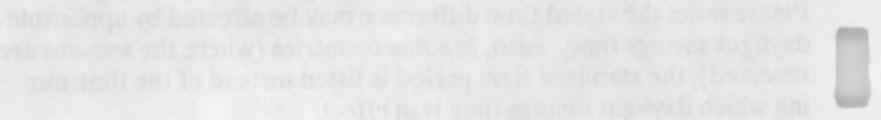

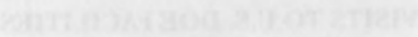

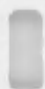

-

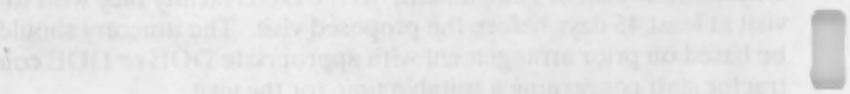

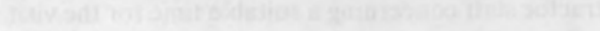

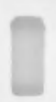

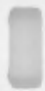

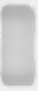

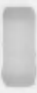

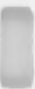

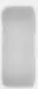




\section{NATIONAL SUMMARIES}





\section{ARGENTINA}

\section{MAJOR PUBLIC HOLIDAYS (1994)}

$\begin{array}{lrlrrl}\text { Jan } & 1 & \text { New Year } & \text { Jun } & 20 & \text { Flag Day } \\ \text { Mar } 31 & \text { Holy Thursday } & \text { Jul } & 9 & \text { Independence Day } \\ \text { Apr } 1 & \text { Good Friday } & \text { Ang } & 15 & \text { Gen. San Martin } \\ \text { May } 1 & \text { Labor Day } & \text { Oct } & 10 & \text { Columbus Day } \\ \text { May } 25 & \text { Revolution Anniversary Dec } & 8 & \text { Immacnlate Conception } \\ \text { Jun } & 10 & \text { Sovereignty } & \text { Dec } & 25 & \text { Christmas }\end{array}$

\section{TIME}

Standard Time Washington, D.C. (Buenos Aires) Standard Time Period:

\section{PASSPORT/VISA}

A passport is needed to depart and re-enter the U.S. Businessrelated travel to Argentina currently does not require a visa; however, it is recommended to consult a travel agency for up-to-date information concerning requirements.

\section{CURRENCY EXCHANGE RATE}

1 U.S. $\$=.99$ Peso

per Wall Street Journal, 01/31/94. As rates fluctnate daily, it is recommended to obtain current rates from local banks or newspapers prior to departure.

\section{DIRECT DIALING}

Individual numbers for direct dial to Argentina are complete as listed, after dialing international access code: 011. Country code is 54; listed local numbers include city code.

\section{U.S. EMBASSY • BUENOS AIRES}

American Embassy 4300 Colombia 1425 Buenos Aires Argentina Tel: $\quad 54-1-777-4533 / 4$ Fax: 54-1-777-0197

Science Counselor

Kenneth D. Cohen 
Population

1993

33 million

\section{ENERGY}

$\begin{array}{lrrl}\text { Electric Power Capacity } & 1993 & 14.3 & \text { GWe } \\ & & 7 \% & \text { nuclear } \\ & 1996 & 17.4 & \text { GWe } \\ & 2000 & 9 \% & \text { nuclear } \\ & & 8 \% & \text { GWe } \\ & & & \\ & & 51.2 & \text { TWh } \\ \text { Electric Power Production } & 1993 & 14 \% & \text { nuclear } \\ & 1996 & 17 \% & \text { nuclear } \\ & 2000 & 13 \% & \text { nuclear }\end{array}$

\section{NUCLEAR POWER}

Policy: Pressurized heavy water reactors (PHWR) with natural uranium and indigenous fuel cycle; currently government ownership and operation of all nuclear power plants - other options being evaluated; development of nuclear plants and services export capability.

Nuclear Power Capacity 1993
1995
2000

Reactor Mix
$1.0 \mathrm{GWe}$

1.6 GWe

$1.6 \mathrm{GWe}$

\section{INDUSTRIAL FUEL CYCLE}

Policy: Develop all phases of the PHWR fuel cycle, gaseous diffusion capability for $\mathrm{U}$ enrichment (Pilcaniyeu), and $\mathrm{D}_{2} \mathrm{O}$ production; may export Pu to nations with breeder reactors. Interim $\mathrm{AR}$ and AFR storage of spent fuel.

Waste Management Stralegy: Options for reprocessing spent fuel analyzed, including vitrification of HLW and disposal of HLW glass canisters in granite host-rock repository, but no decision made. 
Disposal of reduced volumes of LLW and short-lived ILW in nearsurface engineered facilities. No decision made yet on disposal of long-lived ILW.

$\begin{array}{lll}\text { Cumulative SF Arisings (HWR) } & 1993 & 1,900 \text { tHM } \\ & 2000 & 3,300 \text { tHM }\end{array}$

\section{Demonstration/Production Activities}

- $\mathrm{D}_{2} \mathrm{O}$ production (200 t/a): $\mathrm{D}_{2} \mathrm{O}$ enrichment plant to start 1994.

- Uraninm mining and milling (t/a): 1987-100; 1993--130.

- Uranium enrichment (kg/a): Capacity being redefined.

- Conversion of yellowcake to $\mathrm{UO}_{2}$ : fabrication of $\mathrm{UO}_{2}$ fuel ( $\left.\mathrm{t} / \mathrm{a}\right)$ : 1993--200; 1995--300.

- Fuel fabrication: Two of three planned fabrication lines produce 5,880 elements/year.

\section{Major Milestones}

- Dry SF Interim Storage (Embalse, Córdoba)

- HLW Geologic Repository (under study)

- MLW Interim Storage Plant (Ezeiza Atomic Center)

- Atucha II, Nnclear Power Plant

- MLW Near-Surface Engineered Disposal Facility

\section{INTERNATIONAL RELATIONSHIPS}

Member of LAEA; has not signed non-proliferation treaty (NPT); Treaty of Tlatelolco has been signed and ratified. Quadripartite safeguards agreement signed and ratified. 


\section{ORGANIZATION}

- CNEA (Comisión Nacional de Energia Atómica)--National Atomic Energy Commission--owns and operates all nuclear facilities.

\section{CNEA (National Atomic Energy Commission)}

Comisión Nacional de

Energia Atómica (CNEA)

Headquarters

Avenida del Libertador 8250

1429 Buenos Aires, Argentina

Tel: $\quad 54-1-704-1000$

Fax: 54-1-704-2045

President

Regulatory Authority

Nuclear Fuel Cycle

Dr. Manuel A. Mondino

Dr. Dan J. Beninson

Roberto Cirimello

\section{CNEA EZEIZA}

Comisión Nacional de

Energia Atómica (CNEA)

Centro Atómico Ezeiza

Tel: $\quad$ 54-1-480-0141

1842 Ezeiza, Argentina

Fax: $\quad 54-1-480-0815$

Location: 30 km southwest of Buenos Aires, near airport.

Deputy

Regulatory Affairs

LLW Radioactive Waste Mgmt.
Dr. Roberto Marquez

Pedro Sajaroff

Dr. Jaime Pahissa Campá

\section{Facilities}

- Fuel fabrication: the first of three planned fabrication lines started up 1982, second line 1985; produce 380 elements/yr for Atucha I and 5,500 elements/yr for Embalse; third line to produce Atucha II fuel elements.

$$
\text { (contd next page) }
$$




\section{CNEA EZEIZA (contd)}

- Fuel reprocessing: Ezeiza pilot plant, planned capacity of 20 $\mathrm{kgU} / \mathrm{d}$ feed, 10-15 kgPu/a product; non-radioactive runs--1990; hot startup--1994. Potential expansion of pilot plant to commercial facility or new plant with $160 \mathrm{~kg} / \mathrm{d}$ (40 MTU/yr) capacity once planned for late $1990 \mathrm{~s}$. Construction has been put on indefinite hold. 


\section{AUSTRALIA}

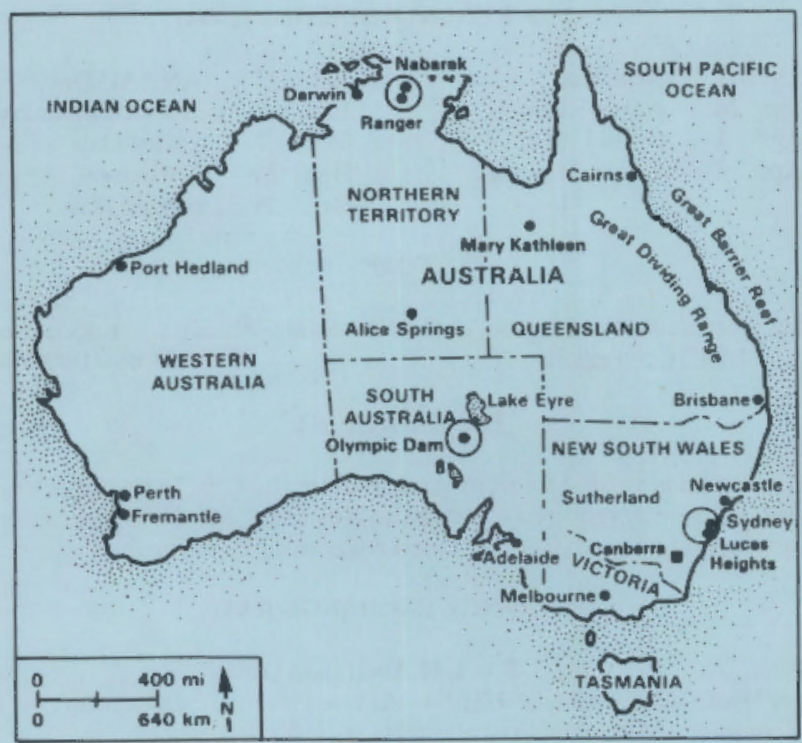




\section{AUSTRALIA}

\section{MAJOR PUBLIC HOLIDAYS (1994)}

$\begin{array}{lrl}\text { Jan } & 1 & \text { New Year } \\ \text { Jan } & 26 & \text { Australia Day } \\ \text { Apr } & 1 & \text { Good Friday } \\ \text { Apr } & 4 & \text { Easter Monday }\end{array}$
Apr 25
Jun 7
Oct 7
ANZAC Day
Dec 25
Queen's Birthday
Dec 26
Labor Day
Christmas
Boxing Day

\section{TIME}

Standard Time Washington, D.C. (New South Wales) +15 hours Standard Time Period:

\section{PASSPORT/VISA}

A passport is needed to depart and re-enter the U.S.; in addition, a visa is currently required for a visit to Australia. Most travel agencies can provide up-to-date information concerning requirements.

\section{CURRENCY EXCHANGE RATE}

\section{U.S. $\$=1.41$ Australian Dollar}

per Wall Street Journal, 01/31/94. As rates fluctuate daily, it is recommended to obtain current rates from local banks or newspapers prior to departure.

\section{DIRECT DIALING}

Individual numbers for direct dial to Australia are complete as listed, after dialing international access code: 011 . Country code is 61; listed local numbers include city code.

\section{U.S. EMBASSY - CANBERRA}

American Embassy

Moonah Place

Canberra, A.C.T. 2600

Tel: $\quad 61-6-270-5000$

Australia

Fax: $\quad 61-6-270-5970$

Scientific Attaché

Zachary Z. Teich 
PNL-9450-1

AUSTRALIA

Population

1992

17 million

ENERGY

$\begin{array}{llll}\text { Electric Power Capacity } & 1992 & 34.5 & \text { GWe } \\ & 1995 & 36.3 & \text { GWe } \\ & 2000 & 37.9 & \text { GWe }\end{array}$

Electric Power Production $1992 \quad 156.4$ TWh

$79 \%$ coal

$10 \%$ hydro

$9 \%$ gas

$2 \%$ oil

\section{NUCLEAR POWER}

Policy: No nuclear power installed; none planned. Large uranium reserves; uranium currently produced for export. Government sponsors nuclear waste management R\&D.

\section{INTERNATIONAL RELATIONSHIPS}

Member of IAEA and OECD/NEA; cooperative agreements for radioactive waste management $R \& D$ (including development of the SYNROC process) with Japan, Italy and the U.K. Memoranda of Understanding, covering SYNROC R\&D, signed with China and the USSR/CIS.

Bilateral safeguards agreements (controlled use of Australian-derived uranium) with Japan, Republic of Korea, Philippines, U.S., Canada, U.K., France, Switzerland, Sweden, Finland, Egypt, Russia/CIS, and Euratom (EC). 


\section{ORGANISATION}

- ANSTO--Australian Nuclear Science and Technology Organisation and Lucas Heights Research Laboratory

\section{ANSTO - LUCAS HEIGHTS}

Australian Nuclear Science and Technology Organisation

New Illawarra Rd, Lucas Heights

Private Mail Bag 1

Menai NSW 2234

Australia

Tel: $\quad 61-2-717-3111$

Fax: $\quad 61-2-543-5097$

Location: Approx. $30 \mathrm{~km}$ SW of Sydney (taxi from Kingsford Smith International Airpori).

$\begin{array}{ll}\text { Executive Director } & \text { Dr. Helen Garnet (A) } \\ \text { Chairman } & \text { C. Ralph Ward-Ambler } \\ \text { General Manager, Scientific } & \text { Dr. Helen Garnett } \\ \text { Environnental Science } & \text { Dr. Wally Zuk } \\ & \text { Fax: 61-2-543-9260 } \\ \text { Environmental Chemistry } & \text { Dr. Richard Lowson } \\ \text { Environmental Physics } & \text { Dr. Ian Ritchie } \\ \text { Chemical Waste Engineering } & \text { Dr. Des Levins } \\ \text { Economical Impacts } & \text { Dr. Peter Airey } \\ \text { Advanced Materials } & \text { Dr. Adam Jostsons } \\ & \text { Tel: 61-2-717-3265 } \\ \text { Materials Assessment } & \text { Fax: 61-2-543-7179 } \\ \text { Waste Conditioning } & \text { Robert Harrison } \\ \text { Operations } & \text { Dr. E. R. Vance } \\ \text { Materials Science } & \text { Alan Ridal } \\ \text { Engineering } & \text { Dr. C. J. Ball } \\ \text { Nuclear Technology } & \text { Don. J. Mercer } \\ & \text { George Malosh } \\ \text { Nuclear Services } & \text { Fax: 61-2-543-9263 }\end{array}$

(contd next page) 


\section{ANSTO - LUCAS HEIGHTS (contd)}

Function: Fuel cycle R\&D--HLW immobilization (SYNROC process development and waste form properties), mill tailings treatment, actinide transport, surface hydrology, and radionuclide release.

\section{Facilities}

- Non-radioactive SYNROC Demonstration Plant Mission: Engineering-scale tests of SYNROC process to provide data for a conceptual radioactive SYNROC plant design by mid1992.

Design Basis: $10 \mathrm{~kg} / \mathrm{h} \mathrm{SYNROC}$; all operations compatible with remote handling; highly instrumented and partly automated.

History: Startup, $5 / 88$ (integrated operation of all steps; three days of operation per month since), upgraded in 1990.

- SYNROC Glove Box Line Mission: Produce SYNROC containing actinides ${ }^{\rho 9} \mathrm{Tc}$. Process Scale: Hundreds of grams/batch. History: Startup, 1984. Refurbished, 1991.

- Hot-Cell Processing Line for SYNROC Mission: Produce SYNROC containing beta/gamma-active fission products.

Process Scale: Hundreds of grams/batch. History: Startup, 1984.

\section{- Semi-Dry Mixer/Rotary Calciner}

Mission: Detailed process improvements on mixing/calcining nitrate/powder.

Design Basis: $5 \mathrm{~kg} / \mathrm{h}$ with in-mixer drying to reduce the size of the rotary calciner.

History: Startup, 1988.

(contd next page) 


\section{ANSTO - LUCAS HEIGHTS (contd)}

Facilities (contd)

- Alkoxide Powder Preparation Facility Mission: Provide fine powders for mixing with nuclear waste slurry.

Design Basls: $100 \mathrm{~kg} / \mathrm{d}$.

History: Starup, 1987; upgraded, 1989.

- Advanced Ceramics Fabrication Laboratory - with full analytical and materials characterization capability. HIP/CIP.

- Engineering Plant Design Team - with 3-D finite element stress analysis, HP/Apollo computers and CAD/CAM.

\section{$\underline{\text { ANU }}$}

Australian National University

P.O. Box 4

Canberra 2600, Australia

Tel: $\quad 61-6-249-4228$

Fax: 61-6-249-5989

Director

Dr. Sue Kesson

Waste Management R\&D: HLW immobilization (SYNROC process). 


\section{BELARUS}

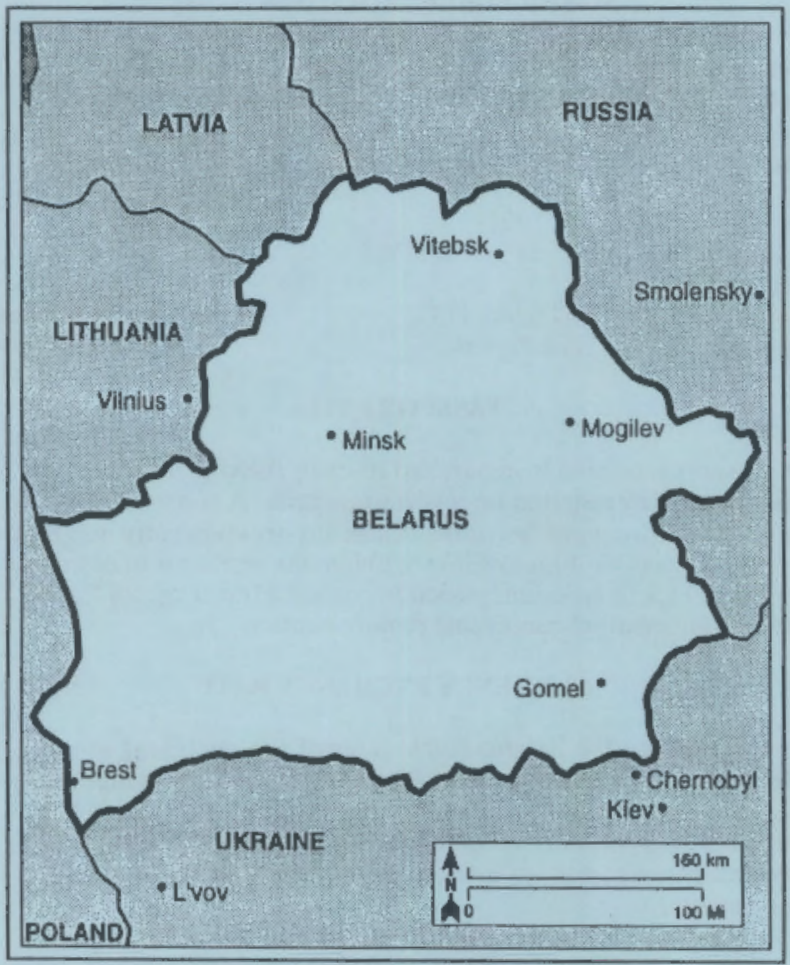




\section{BELARUS}

MAJOR PUBLIC HOLIDAYS (1994)

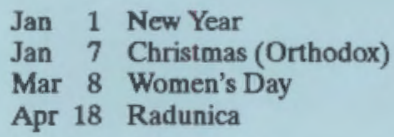

Jan 1 New Year

Jan 7 Christmas (Orthodox)

Mar 8 Women's Day

Apr 18 Radunica
May 1 Labor Day
May 9 Victory Day
Jul 27 Independence Day
Nov 2 Memorial Day
Dec 25 Christmas

\section{TIME}

Standard Time Washington, D.C. +8 hours Daylight Savings Time Period:

\section{PASSPORT/VISA}

A passport is needed to depart and re-enter the U.S. In addition, a visa is currently required for a visit to Belarus. A visa is available, with certain prerequisites, at the Minsk airport upon entry into the country; however, it is advisable to obtain the visa prior to departure from the U.S. It is recommended to consult a travel agency for upto-date information concerning requirements.

\section{CURRENCY EXCHANGE RATE}

Local currency, the Belarus Ruble (note of National Bank of Belarus), is only available upon entry into Belarus. Exchange rates cannot be quoted due to continuing fluctuations. Payment in U.S. currency is apparently acceptable in many places. It is strongly recommended to consult with the U.S. embassy for up-to-date information.

\section{DIRECT DIALING}

Individual numbers for direct dial to Belarus are complete as listed, after dialing international access code: 011 . Country code is 7; listed local numbers include city code.

\section{U.S. EMBASSY - MINSK}

American Embassy

ul. Starovilenskaya 46

Minsk, Belarus

Economic Section
Tel:

$$
\begin{gathered}
7-0172-34-6537 \text { or } \\
-7761 \\
7-0172-31-5000
\end{gathered}
$$

Fax: 7-0172-34-7853

George Krol 
Population

1993

10 million

\section{ENERGY}

Electric Power Capacity

$\begin{array}{rrl}1992 & 7.0 & \mathrm{GWe} \\ 2000 & 8.6 & \mathrm{GWe} \\ & .0 \% & \text { nuclear } \\ 2005 & 9.3 & \mathrm{GWe} \\ & >8 \% & \text { nuclear }\end{array}$

Electric Power Production 1992

$\begin{aligned} 38.7 & \text { TWh } \\ 55 \% & \text { oil } \\ 40 \% & \text { gas } \\ <3 \% & \text { coal/peat } \\ <2 \% & \text { solids } \\ 0 \% & \text { nuclear } \\ 5 \% & \text { nuclear }\end{aligned}$

\section{NUCLEAR POWER}

Though Belarus currently produces no nuclear power, approximately $25 \%$ of its total electricity consumption is provided by nuclear power plants at Ignalina in Lithuania and Smolensk in Russia. Construction of a nuclear power plant at Minsk, with projected capacity of 2,000 MWe and planned additional capacity of $6,000 \mathrm{MWe}$, was halted in 1986 due to the events at Chemobyl. The current government has stated that nuclear power is a necessity in the future of Belarus.

$\begin{array}{lcrl}\text { Nuclear Power Capacity } & 2005 & 1.0 & \text { GWe } \\ \text { Reactor Mix } & 2005 & \text { PWR } & 1(2005)\end{array}$

\section{INDUSTRIAL FUEL CYCIE}

Policy: Because Belarus has no uranium natural resources, no uranium enrichment is foreseen, nor is fuel reprocessing.

Waste Management Strategy: A waste management concept and strategy for disposal of waste from the planned first Belarus NPP is now being developed. LLW generated during operation and from decommissioning of a research LWR (IPEP) was managed in accordance with known regulations in the former Soviet Union. This waste 
was stored in an engineered structure in an underground facility near Sosny that is also used for spent radioactive sources. Spent fuel from decommissioning the research LWR was sent to Russia for reprocessing.

\section{INTERNATIONAL RELATIONSHIPS}

Member of LAEA; Belarus has signed and ratified the NPT in 1993.

\section{ORGANIZATION}

Government Responslbilities --

Nuclear Fuel Cycle/Waste Management

- Minlstry of Power Engineering

- controls all electric power production and its industrial consumption operates electric power network

- provides and conducts the National Power Development Program

- Gospromatomnedzor (State Committee on Supervision of Industrial and Nuclear Safety--regulations, controls, licensing of nuclear installations.

- Goscomchernobyl (State Chernobyl Committee)--responsible for National Chemobyl Program.

- GoscomecologlJ (State Committee on Environment Protection)-regulations, inspections.

\section{INDUSTRIAL/UNIVERSITY RESPONSIBILITIES}

- Bel NIPI Energoprom (National Research and Design Institute)-develops technical policy for electric power/energy resources.

- GSP POLESJE (State Specialized Enterprise/State Chemobyl Committee)-decontamination of contaminated zone in southern Belarus. 
- IPEP (Institute of Power Engineering Problems/Academy of Sciences)-waste management $R \& D$ and reactor physics/engineering.

- IREP (Institute of Radio-Ecological Problems/Academy of Sciences)--research related to radionuclide migration in biosphere, nuclear medicine, radiochemistry.

- DD\&PM (Design Department \& Pilot Manufacture/Academy of Sciences)-academic research association.

\section{BEL NIPI ENERGOPROM}

Belarus Research and Design Institute of Power Engineering lndustry

ul. Romanovskaja sloboda 5A 220048 Minsk, Belarus Tel: $\quad 7-0172-26-5277$ Fax: $\quad 7-0172-20-5317$

Director Ivan P. Sporta

Function: Development of technical policy for electric power/energy resources and of electric power network installations.

\section{GOSPROMATOMNADZOR}

State Committee on Supervision of Industrial/Nuclear Safety
ul. Chkalova 6
220039 Minsk, Belarus
Tel: $\quad$ 7-0172-24-4119
Fax: $\quad 7-0172-24-3700$

Chairman

Nuc./Rad. Safety Inspection

Alexander B. Zujev

Peter V. Bulyga

Functlon: Responsible for regulations, control, and licensing of nuclear installations and radiation-emitting facilities. 


\section{GOSCOMCHERNOBYL}

State Chernobyl Committee

ul. Lenin 14

Tel: $\quad$ 7-0172-27-4987

220030 Minsk, Belarus

Fax: $\quad 7-0172-29-3439$

Chairman

Dep. Chairman, WM/Science/

Ivan A. Kenik

R\&D, Internatl. Relations

Dr. Igor V. Rolevich

7-0172-27-0770

Board on Protective

Measures/D\&D/WM

Dr. Gennady V. Antzypov

7-0172-27-0762

Function: Regulate, control, and finance the National Chernobyl Program; licensing of decontamination/waste management activities for area affected by the Chernobyl fallout.

\section{GOSCOMECOLOGLA}

State Committee on

Environmental Protection

ul. Kollektornaja 10

220048 Minsk, Belarus

Tel: $\quad 7-0172-20-6691$

Fax: $\quad 7-0172-20-5583$

Chairman

Anatoly M. Dorofejev

Function: Regulatory and inspection authority.

\section{GSP POLESJE}

State Specialized

Enterprise "POLESJE"

ul. Karpovich 11

Tel: $\quad$ 7-0172-53-1584

246017 Gomel, Belarus

Fax: $\quad 7-0172-53-7486$

Function: Decontamination of affected zone in southern Belarus; treatment and conditioning of waste generated as a result of decontamination. 


\section{IPEP}

Institute of Power Engineering

Problems

Belarus Academy of Sciences

Sosny

Tel: $\quad$ 7-0172-26-0698

220109 Minsk, Belarus

Fax: $\quad$ 7-0172-26-3743

Director

Material Properties/

Prof. A. A. Mikhalevich

WM Technologies

Dr. Alexandre J. Grevenkov

Nuclear Reactor Physics

7-0172-46-7142

Dr. Igor A. Savushkin

7-0172-46-7434

Function: Waste management R\&D - LLW/LLW immobilization, liquid LLW treatment, thermal/chemical processing of radioactive wood waste.

Facilities:

- Pilot Plant for LLW/LWW Immobllization

- Pilot Installations for LLLW Treatment/Conditioning

\section{$\underline{\text { IREP }}$}

Institute of Radio-Ecological

Problems

Belarus Academy of Sciences

Sosny

220109 Minsk, Belarus

Tel: $\quad 7-0172-46-7253$

Fax: $\quad 7-0172-46-7615$

Director

Radiochemistry

Prof. George A. Sharovarov

Dr. Yuri P. Davydov

Function: R\&D on radionuclide migration in biosphere, decontamination, conditioning of liquid LLW (generated after remediation of contaminated site), nuclear medicine, radiochemistry. 


\section{SOSNY (Academic Research Association)}

ANTK Sosny

Belarus Academy of Sciences

Sosny

220109 Minsk, Belarus

Tel: $\quad 7-0172-46-7512$

Fax: $\quad 7-0172-46-7615$

Director General

Dr. Sergey E. Chigrinov

Location: $24 \mathrm{~km}$ southwest of Minsk, $2 \mathrm{~km}$ from Sosny settlement.

Function: Academic research association, includes IPEP, IREP, IRPCP, DD\&PM. Former Nuclear Power Engineering Institute of the USSR Academy of Sciences (1965-1992).

\section{DD \& PM}

Design Department

with Pilot Manufacture

Sosny

Tel: $\quad$ 7-0172-46-7538

220109 Minsk, Belarus

Fax: $\quad 7-0172-46-7403$

Director

Dr. Vladimir A. Kosterov

Function: Design/production of pilot installations related to nuclear power plant, engineering, waste management technologies.

Specialized Enterprise "Sosny" Tel: 7-0172-46-7539

Director

Victor B. Ivanov

Function: Spent nuclear materials storage in engineered shallowground facility. 


\section{BELGIUM}

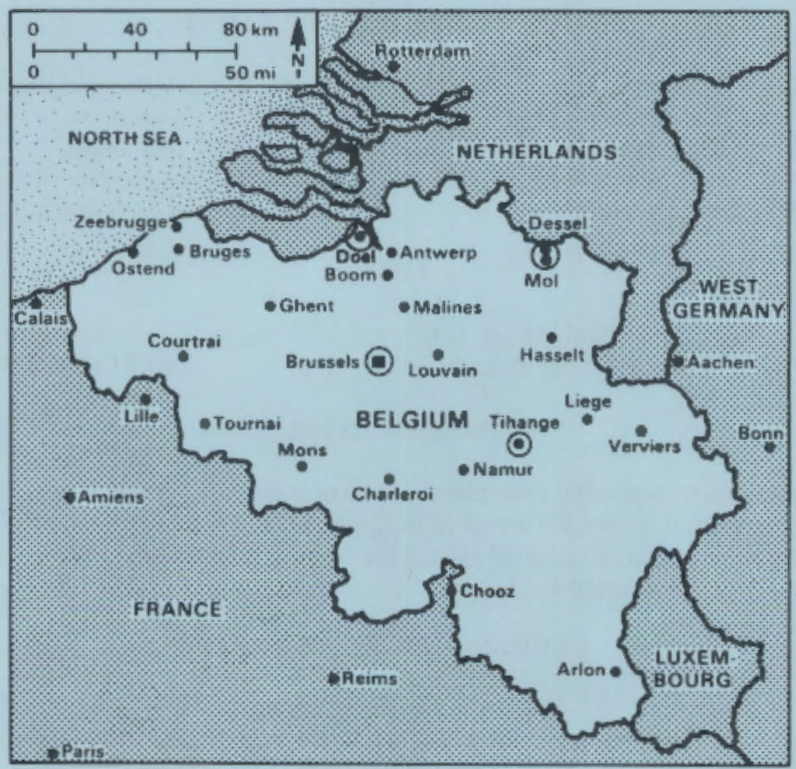




\section{BELGIUM \\ MAJOR PUBLIC HOLIDAYS (1994)}

Jan 1 New Year

Apr 4 Easter

May 1 Labor Day

May 12 Ascension

May 23 Pentecost
Jul 21 National Day

Aug 15 Assumption

Nov 1 All Saints

Nov 15 Armistice

Dec 25 Christmas

\section{TIME}

Standard Time Washington, D.C.:

+6 hours

Daylight Savings Time Period:

$03 / 27 \cdot 09 / 24 / 94$

\section{PASSPORT/VISA}

A passport is needed to depart and re-enter the U.S. A visa is currently not required for a visit to Belgium; however, it is recommended to consult a travel agency for up-to-date information concerning requirements.

\section{CURRENCY EXCHANGE RATE}

1 U.S. $\$=35.61$ Franc

per Wall Street Journal, 01/31/94. Because rates fluctuate daily, it is recommended to obtain current rates from local banks or newspapers prior to departure.

\section{DIRECT DIALING}

Individual numbers for direct dial to Belgium are complete as listed, after dialing international access code: 011 . Country code is 32; listed local numbers include city code.

\section{U.S. EMBASSY - BRUSSELS}

American Embassy

27 Boulevard du Regent

1000 Brussels

Belgium

Tel: $\quad 32-2-513-3830$

Economics Section

Fax $\quad 32-2-511-2725$

John L. Moran 
PNL-9450-1

Population

1992

9.5 million

\section{ENERGY}

Electric Power Capacity

$\begin{array}{lll}1992 & 14.1 & \text { GWe } \\ & 39 \% & \text { nuclear } \\ 1995 & 15.1 & \text { GWe } \\ & 36 \% & \text { nuclear } \\ 2000 & 16.1 & \text { GWe } \\ & 34 \% & \text { nuclear }\end{array}$

Electric Power Production 1992

71.4 TWh

$61 \%$ nuclear

$26 \%$ coal

$10 \%$ gas

$2 \%$ oil

$<1 \%$ hydro

1995

$54 \%$ nuclenr

2000

54\% nuclear

\section{NUCLEAR POWER}

Policy: Produce base load electricity by nuclear and coal power plants. Decided against adding proposed eighth (1300 MWe) nuclear unit (at least during next few years).

Nuclear Power Capacity 1992 2000

$5.5 \mathrm{GWe}$ 5.5 GWe

Reactor Mix 1993 PWR 7 (1975-85) 


\section{INDUSTRIAL FUEL CYCLE}

Policy: Well-rounded capability--uranium enrichment (share in Eurodif); $\mathrm{MOX}$ and $\mathrm{UO}_{2}$ fuel fabrication; purchase of foreign reprocessing services; decision made to dismantle former Eurochemic reprocessing plant.

Waste Management Strategy (responsibility of ONDRAF): Vitrify HLW and store 50 years (investigation of HLW, ILW and LLW disposal in clay formations underway); treat and immobilize other wastes; sea-dumping of LLW halted; shallow-ground disposal of LLW under investigation.

$\begin{array}{lll}\text { Cumulative SF Arisings (LWR) } & 1990 & 1,290 \mathrm{tU} \\ & 2000 & 3,000 \mathrm{tU}\end{array}$

\section{Major Milestones}

- Selection/characterization of site for LLW disposal facility

- Storage facility for waste from Belgian fuel reprocessed abroad

- Safety assessment/feasibility report for demonstration of HLW disposal operations in proposed clay repository

- Construction start of HLW repository

- Disposal start of HLW

\section{INTERNATIONAL RELATIONSHIPS}

\section{DOE/SCK Agreement in the Field of Radioactive Waste Management}

Term: $01-19-81$ to $01-19-94$.

Scope: Final disposal in geologic formations; retrievable storage; waste processing; environmental effects; emphasis on technology information exchange.

Member of EC, IAEA, OECD/NEA; partnership in Eurodif uranium enrichment plant (France); Belgian URL at Mol is cosponsored by CEC. 


\section{OORGANIZATION}

MINISTRY OF ECONOMIC AFFAIRS

PRIVATE

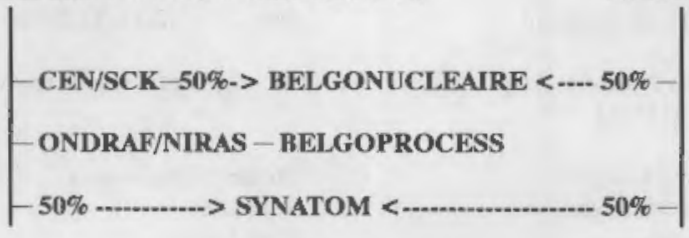

INTERESTS

\section{BELGONUCLEAIRE}

Belgonucleaire S.A.

Avenue Ariane 2-4

Tel: $\quad 32-2-774-0511$

1200 Brussels, Belgium

Fax:

32-2-774-0547

General Director

Georges Cornet

Functlon: Provide engineering services for nuclear power plants, nuclear fuel cycle facilities, and waste treatment plants; fabricate MOX fuels.

Owner: CEN/SCK (50\%), utilities/holding companies (50\%).

MOX Plant

Europalaan 20

2480 Dessel, Belgium

Tel: $\quad 32-14-33-0211$

Fax: $\quad 32-14-31-7046$

Manager

Jean Van Vliet

Function: Produce MOX fuels ( $35 \mathrm{t} / \mathrm{a}$ for LWR or $10 \mathrm{t} / \mathrm{a}$ for FBR). 
Belgoprocess

Gravenstraat 73

2480 Dessel, Belgium

Tel: $\quad 32-14-33-4111$

Fax: $\quad 32-14-31-3012$

[Brussels National Airport (Zaventem); then by rental car or train $(1.5 \mathrm{~h})$ to $\mathrm{Mol}$.]

Managing Director
General Manager
Operations
Decommissioning
Safety

\author{
Robert Vandenplas \\ Jef Claes \\ Paul Luycx \\ Lucien Teunckens \\ Jean Paul Deworm
}

Activities: Maintenance/dismantling of ex-Eurochemic reprocessing facilities and obsolete waste treatment facilities formerly belonging to CEN/SCK; treatment/conditioning of all categories of low-, medium-, and high-level waste; from 1986 to 1991 joint operation with WAK of Pamela vitrification plant, now being kept in stand by for potential future vitrification of HLLW from WAK pilot reprocessing plant at Karlsruhe, Germany.

\section{Owner: ONDRAF/NIRAS}

\section{Facilities}

- Eurobitum (bituminization plant) Mission: Immobilize ILW.

Design Basis: Batch chemical pretreatment; screw extruder-evaporator (continuous); capacily, $650 \mathrm{~m}^{3} / \mathrm{a}$ ILW History: Startup, 1978; on-line time, 87\% through June 1983. Plant now operated as needed.

- Eurowatt (hot pilot plant-solvent treatment) Mission: Treat PUREX (TBP-kerosene) solvent.

Design Basis: Extract TBP with concentrated $\mathrm{H}_{3} \mathrm{PO}_{4}$, pyrolyze $\mathrm{H}_{3} \mathrm{PO}_{4}$ fraction; capacity, $1 \mathrm{~m}^{3} / \mathrm{d}$.

History: Startup, 1982; now in standby.

(contd next page) 


\section{$\underline{\text { BP }}$ (contd)}

- PAMELA HLLW Vitrification Plant [built by FRG (see under WAK in GE Section) and operated by WAK/Belgoprocess team]. In standby for future vitrification of HLLW from WAK pilot reprocessing plant at Karisruhe.

- LLW Treatment Facilities (formerly of CEN/SCK)

Beta/Gamma Waste Incinerator: capacity of $100 \mathrm{~kg} / \mathrm{h}$ solid waste and $40 \mathrm{l} / \mathrm{h}$ of organic liquids. Combustion temperature of $900^{\circ} \mathrm{C}$. Overall mass reduction factor is 20 ; combustion efficiency $>99.9 \% ;>600$ t of waste throughput since early 1960 s. Planned shutdown 1994.

High-Temperature Slagging Incinerator: shutdown 1988.

Water Treatment Facllity: capacity of $>200,000 \mathrm{~m}^{3} / \mathrm{a}$; puritication by flocculation; discharge of purified water to the river, conditioning of sludges into bitumen.

\section{- Low-Level Solid Waste Treatment Facility}

Startup expected in second half of 1994; new integrated facility, including receptiou of waste and buffer storage, size reduction unit; supercompaction, incineration at $900^{\circ} \mathrm{C}$, supercompaction of ashes and cementation of all treated LLW.

\section{FBFC (Fuel Fabrication Company)}

FBFC International

Europalaan 12

2480 Dessel, Belgium

Managing Director

Plant Manager
Tel:

Fax:

32-14-31-5845

Henri Potdeviu

Patrick Van denhove

Function: Fabrication of fuel assemblies for LWR (capacity: $300 \mathrm{t} / \mathrm{a}$, uranium and MOX fuel). Belgian subsidiary of French-owned Société Franco-Belge de Fabricatiou de Combustibles. 


\section{MINISTRY OF ECONOMIC AFFAIRS}

Ministry of Economic Affairs

Administration of Energy

Rue de Mot, 30

Tel: $\quad 32-2-233-6111$

1040 Brussels, Belgium

Fax: $\quad 32-2-930-4138$

\section{MINISTRY OF PUBLIC HEALTH AND ENVIRONMENT}

Ministère de la Santé Publique et de l'Environnement

Quartier Vésale 2-3

Tel: $\quad 32-2-210-4966$

1010 Brussels, Belgium

Fax: $\quad 32-2-210-4967$

ONDRAF/NIRAS (National Onganization for Radioactive Wastes and Fissile Materlals)

Organisme National des Déchets

Radioactifs et des Matières

Fissiles (ONDRAF/NIRAS)

Place Madou 1, B.P. 24

Tel: $\quad 32-2-212-1011$

1030 Brussels, Belgium

Fax: $\quad 32-2-218-5165$

Chairman, Board of Directors

J. P. Poncelet

Chair., Perm. Tech. Committee

F. Deconinck

General Manager

F. Decamps

\section{Owner: Government.}

Function: Define Belgian waste management policy and $R \& D$ requirements; responsible for transportation of radioactive materials, waste treatment,conditioning and interim storage, spent fuel AFR storage, waste disposal, fissile material storage.

The organization is governed by a Board of Directors composed of a President, Vice-President, and Board members representing various national ministries and local government executives. The Board is advised by a Permanent Technical Committee. 


\section{SCK/CEN (Nuclear Energy Research Cenier)}

Studiecentrum voor Kemenergie

Centre d'Étude de l'Énergie

Nucléaire

Laboratories

Boeretang 200

$2400 \mathrm{Mol}$, Belgium

Tel: $\quad 32-14-33-2111$

Fax: $\quad 32-14-31-5021$

Chairman of the Board

General Manager

Waste/Disposal

Decommissioning

Prof. Roger E. Van Geen

Carl M. Malbrain

Bernard Nerdael

Guy Collard

Owner: Government--Ministry of Economic Affairs.

Waste Management R\&D: Geologic waste isolation in clay formations, waste treatment (decontamination and recycling of boric acid, removal of plutonium from waste generated by fuel fabrication, etc.), decommissioning (decontamination, dismantling, restoration) of nuclear facilities.

\section{Facilities}

- BR3 Decommissioning Project

Mission: Optimization of the decommissioning of PWRs; radiological, technical, and financial management of decommissioning, applied on an actual PWR; all components of a power plant and all techniques to be used in decommissioning.

Process: Internals are being dismantled; comparison of immediate and delayed decommissioning; optimum application.

(contd next page) 


\section{SCK/CEN (contd)}

\section{- HADES Underground Research Laboratory}

Mlssion: In-situ investigation to demonstrate the feasibility, construction, safety, and acceptability of disposal of ILW, TRU waste, and HLW in a deep clay formation.

Description: Access shaft to 230 -m level, $2.65 \mathrm{~m}$ useful diameter; laboratory gallery, $3.5 \mathrm{~m}$ useful dia. by $30 \mathrm{~m}$ length; cast iron tiner. Demo/hest gallery: $3.5 \mathrm{~m}$ dia., concrete-lined, $65 \mathrm{~m}$ length for large-scale integrated tests.

Test Program: Migration of radionucldes and gas, near-field studies, thermohydraulic behavior, hydrogeochemistry of Boom clay and surrounding water-bearing formation, in-situ tests on waste package components, characterization and compatibility studies of conditioned HLW, performance studies, including shallow-land burial of LLW.

History: Laboratory operational, late 1984.

\section{SYNATOM}

\section{SYNATOM S.A.}

Avenue Marnix, 13

1050 Brussels, Belgium

Chairman, Board of Directors

Managing Director

General Manager

Fuel Reprocessing Service
Tel: $\quad 32-2-505-0711$

Fax: $\quad 32-2-505-0790$

F. Aerns

J. Laurent

Pierre Goldschmidt Jean Danguy

Function: Provide commercial fuel cycle services for the Belgian nuclear utilities.

Owners: Government/SNI (50\%), Electrabel (50\%). 


\section{BRAZIL}

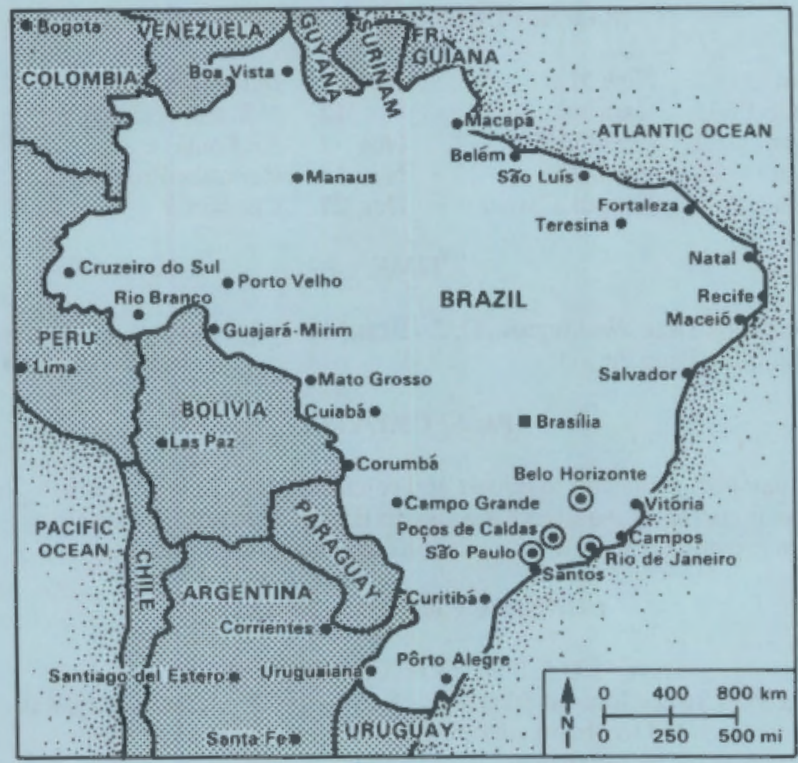




\section{BRAZIL}

MAJOR PUBLIC HOLIDAYS (1994)

$\begin{array}{lrl}\text { Jan } & 1 & \text { New Year } \\ \text { Feb } & 12-15 & \text { Carnival } \\ \text { Apr } & 1 & \text { Good Friday } \\ \text { Apr } & 21 & \text { Tirdentes } \\ \text { Jun } & 2 & \text { Corpus Christi }\end{array}$

Sep 7 Independence

Oct 12 N.S. Aparecida

Nov 2 All Souls

Nov 15 Republic Proclamation

Dec 25 Christmas

\section{TIME}

Standard Time Washington, D.C. (Brasilia) +2 hours Standard Time Period:

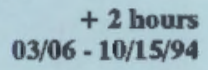

\section{PASSPORT/VISA}

A passport is needed to depart and re-enter the U.S.; in addition, a visa is currently required for a visit to Brazil. Most travel agencies can provide up-to-date information concerning requirements.

\section{CURRENCY EXCHANGE RATE}

1 U.S. $\$=458.65$ Cruzeiro (Cz\$)

per Wall Street Journal, 01/31/94. Because rates fluctuate daily, it is recommended to obtain current rates from local banks or newspapers prior to departure.

\section{DIRECT DIALING}

Individual numbers for direct dial to Brazil are complete as listed, after dialing international access code: 011. Country code is 55; listed local numbers include city code.

\section{U.S. EMBASSY - BRASILIA}

American Embassy

Avenida das Naçóes, Lote 3

CEP 70403, Brasilia

Tel: $\quad 55-61-321-7272$

Brazil

Fax: $\quad 55-61-225-9136$

Science Counselor

Roy C. Simpkins 
Population

1993

150 million

\section{ENERGY}

$\begin{array}{lcrl}\text { Electric Power Capacity } & 1993 & \begin{array}{r}63 \\ 1 \%\end{array} & \text { GWe } \\ & & \text { nuclear } \\ & 1995 & 78 & \text { GWe } \\ 1 \% & \text { nuclear } \\ & & 98 & \text { GWe } \\ 1 \% & \text { nuclear } \\ & 2000 & & \\ \text { Electric Power Production } & 1993 & 220 & \text { TWh } \\ & & 84 \% & \text { hydro/thermal } \\ & & 11 \% & \text { coal/gas } \\ & & 4 \% & \text { oil } \\ & 1 \% & \text { nuclear } \\ & 1995 & 1 \% & \text { nuclear } \\ & 1 \% & \text { nuclear }\end{array}$

\section{NUCLEAR POWER}

Pollcy: Complete nuclear industry with closed fuel cycle, based upon technology transfer from FRG and other countries.

$\begin{array}{lrrl}\text { Nuclear Power Capacity } & 1993 & 0.6 & \text { GWe } \\ & 2000 & 1.9 & \text { GWe } \\ \text { Reactor Mix } & 1993 & \text { PWR } & 1(1984) \\ & & & 2(1997 / 04)\end{array}$

Reactor Development: Low power PWR; research/isotope production reactor (light water/low enrichment); FBR (experimental).

\section{INDUSTRIAL FUEL CYCLE}

Pollcy: Development of full commercial capability for closed fuel cycle-- $\mathrm{U}$ mining and milling; conversion of $\mathrm{U}_{3} \mathrm{O}_{8}$ to $\mathrm{UF}_{6}$; enrichment; $\mathrm{UO}_{2}$ fuel fabrication; fuel reprocessing.

Waste Management Strategy: Not yet defined for HLW; nearsurface disposal for LLW, including the Cs-137 waste from the Goiania accident (1987). 
Cumulative SF Arisings (LWR)

1990

$48 \mathrm{tU}$

1995

162 tU

2000

$-412 \mathrm{tU}$

Demonstration/Production Activities

- Uranium mining and milling: $300 \mathrm{tU}_{3} \mathrm{O}_{4} / \mathrm{a}$--in operation.

- $\mathrm{U}_{3} \mathrm{O}_{8}$ to $\mathrm{UF}_{6}$ conversion: (1984) 90 tU/a; planned expansion delayed indefinitely.

- Uranium enrichment (gas centrifuge): small experimental demonstration (1987).

- Uranium enrichment (Becker nozzle process) at Resende:

- First Cascade, 24 stages; 6 k SWU/a (1985); interrupted in 1993.

- Fuel fabrication: $100 \mathrm{tU} / \mathrm{a}$ (1982); design capacity-400 tU/a.

- Spent fuel reprocessing: $10 \mathrm{~kg} / \mathrm{d}$ pilot plant (1986 startup originally scheduled, currently delayed indefinitely).

\section{INTERNATIONAL, RELATIONSHIPS}

Joint Natural Analogue Studies - Pocos de Caldas Project Joint study by Sweden, Switzerland, U.K., and U.S. of migration of radionuclides from uranium ore deposits in Brazil.

Member of IAEA (has not signed NPT); dependence on nuclear technology transfer from other nations, principally from FRG. Quadripartite agreement signed in 1994 with Argentina, ABACC, and IAEA to implement full-scope safeguards. 


\section{ORGANIZATION}

- Federal Republic--President (Executive), Bicameral National Congress (Legislative), and Supreme Federal Tribunal (Judiciary).

- SAE (Strategic Business Secretariat)--subordinated to the President, responsible for the planning, execution, and control of nuclear power program.

- Eletrobrás (Centrais Eletricas Brasileiras)--Planning/supervision of power plant construction and operation of transmission/ distribution system. Established in 1961 to coordinate activities of state, municipal, and private utilities. Operates through regional subsidiaries, i.e., FURNAS. Also responsible for appropriate R\&D.

- ABACC (Argentina/Brazil Agency for Accounting/Control of Nuclear Materials)--bilateral safeguards agency, established in 1994.

- CNEN (National Nuclear Energy Commission)--regulatory/R\&D. Research Institutes: CDTN, IEN, IPEN, IRD.

- INB (Brazilian Nuclear Industries)--commercial nuclear fuel cycle activities, uranium mining and processing.

\section{CDTN (Center for the Development of Nuciear Technology)}

Centro de Desenvolvimento de Tecnologia

Nuclear de Nuclebras (CDTN)

Rua Gonçalves Dias No. 1054

Belo Horizonte, MG, Brazil

Tel:

Fax:

Superintendent

Fernando Lomeiras

Function: Applied research and industrial development of uses for atomic energy. Thiga reactor (research/isotope production); laboratory scale enrichment nozzle process. 


\section{CNEN (National Nuclear Energy Commission)}

Comissão Nacional de Energia Nuclear (CNEN)

Rua General Severiano 90

Botafogo ZC-82, CEP 22290

Tel: $\quad 55-21-546-2232$

Rio de Janeiro, RI, Brazil

Fax: $\quad 55-21-546-2379$

President

Director, Nuclear Safety

Marcio Costa

Head, Waste Disposal

Laercia Vinnas

Ana Maria Xavier

Function: Regulation, surveillance, and licensing of nuclear reactors, fuel cycle facilities and radiation-emitting installations; promotion of nuclear technology R\&D and technology transfer to private industry; promotion and training of personnel. Controls four research institutes: CDTN, IEN, IPEN, and IRD.

\section{IEN (Nuclear Engineering Institute)}

Instituto de Engenharia Nuclear

Cidade Universitária

Illha do Fundão

Caixa Postal 2186, CEP 20001

Rio de Janeiro, RJ, Brazil

Tel: $\quad$ 55-21-280-3113

Fax: $\quad 55-21-590-2692$

Superintendent

Luiz Arieta

Activities: Nuclear reactor physics, cyclotron radioisotope production, reactor engineering, research reactor operation, metallurgy, nuclear/applied chemistry, nuclear instrumentation (development/ production), health physics, mathematics/computation and sodium technology, reactor development.

\section{Facilities}

- Laboratories for Nuclear Chemistry, Metallurgy and Engineering

- Argonaut-type reactor - $10 \mathrm{~kW}$

- Sodium loop - $300 \mathrm{~kW}$

- Cyclotron 


\section{IPEN (Energy and Nuclear Research Institute)}

Instituto de Pesquisas Energeticas e Nucleares

Cidade Universitária

Caica Postal 11.049

Pinheiros, CEP 01000,

São Paulo, Brazil

Tel: $\quad$ 55-11-211-6011

Fax:

Superintendent

Claudio Rodrigues

Nuclear Activities: Nuclear physics, nuclear medicine, radiobiology, radiation health/safety, engineering/reactor technology/instrumentation, nuclear materials chemistry, isotope and radiation applications/ production, nuclear waste disposal, nuclear metallurgy, radiochemistry.

Facilities

- $\mathrm{U}_{3} \mathrm{O}_{8}-\mathrm{UF}_{6}$ conversion plant at Ipero (90 tU/a)

- SF reprocessing laboratory

- Small experimental gas centrifuge (uranium enrichment)

- LOw-power PWR reactor

- Swimming pool $10 \mathrm{MW}$ reactor (isotope production) 


\section{$\underline{\text { IRD (Health Physics and Dosimetry Institute) }}$}

Instituto de Radioproteção e Dosimetria

Avenida das Américas Km 11.5

Barra Da Tijuca, CEP 22700

Tel: $\quad 55-21-442-9777$

Rio de Janeiro, RJ, Brazil

Fax: $\quad 55-21-442-2950$

Director

Jose Marcos Godoy

Activities: Personal dosimetry control, calibration of radiation detectors, reactor environment control, nuclear medicine and X-ray equipment control, radiobiology, background evaluation, dosimetry research.

\section{Facilities}

- Brazilian Secondary Standards Dosimetry Laboratory 


\section{CANADA}

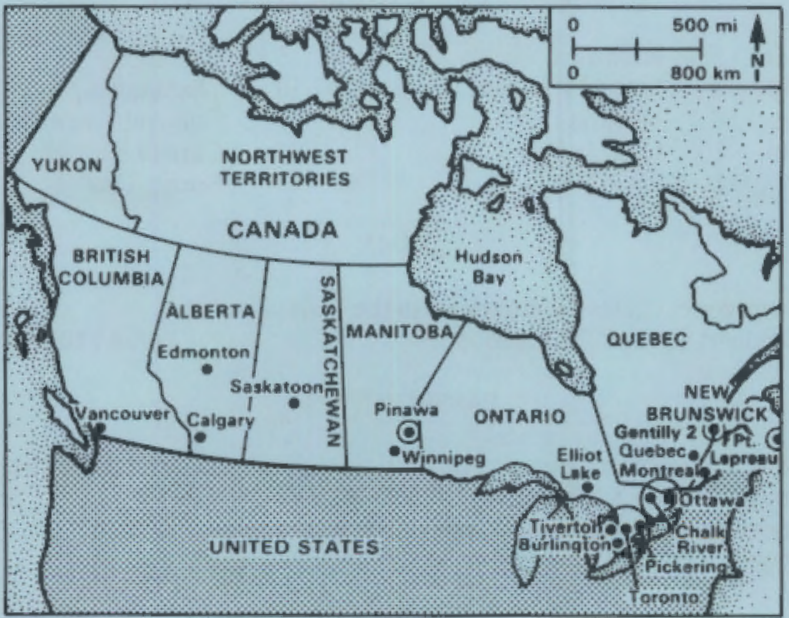


MAJOR PUBLIC HOLIDAYS (1994)

$\begin{array}{lrllrl}\text { Jan } & 3 & \text { New Year } & \text { Sep } & 5 & \text { Labor Day } \\ \text { Apr } 1 & \text { Good Friday } & \text { Oct } & 10 & \text { Thanksgiving } \\ \text { May } 18 & \text { Victoria Day } & \text { Nov } & 11 & \text { Remembrance Day } \\ \text { Jul } 1 & \text { Canada Day } & \text { Dec } & 26 & \text { Christmas } \\ \text { Aug } & 1 & \text { Civic Day } & \text { Dec } & 27 & \text { Boxing Day }\end{array}$

TIME

Time zones correspond to those in the United States.

Daylight Savings Time period:

$04 / 24 \cdot 10 / 30 / 94$

\section{PASSPORT/VISA}

In lieu of passport, proof of U.S. citizenship such as birth certificate (but not driver's license) is sufficient for a visit to Canada. Most travel agencies can provide up-to-date information concerning requirements.

\section{CURRENCY EXCHANGE RATE}

\section{U.S. $S=1.33$ Canadian Dollar}

per Wall Street Journal, 01/31/94. As rates fluctuate daily, it is recommended to obtain current rates from local banks or new/spapers prior to departure.

\section{DIRECT DIALING}

Individual numbers for direct dial to Canada are complete as listed. Dial long distance access code: 1 , followed by three-digit area code plus seven-digit local number.

\section{U.S. EMBASSY - OTTAWA}

American Embassy 100 Wellington Street

Ottawa, ON

K1P 5T1 Canada

Tel: $\quad$ 613-238-5335

Fax: $\quad 613-238-5720$

Science Counselor

Thomas J. Wajda 
$\begin{array}{lll}\text { Population } & 1992 & 27.5 \text { million }\end{array}$

ENERGY

$\begin{array}{lrrl}\text { Electric Power Capacity } & 1992 & 104.5 & \text { GWe } \\ & 1995 & 13 \% & \text { nuclear } \\ & & \text { GWe } \\ & 14 \% & \text { nuclear } \\ & 2000 & 117.2 & \text { GWe } \\ & & 13 \% & \text { nuclear }\end{array}$

Electric Power Production $1992 \quad 501.5$ TWh $62 \%$ hydro/geoth.

$17 \%$ coal

$15 \%$ nuclenr

$3 \%$ oil

$2 \%$ gas

$199519 \%$ nuclear

$2000 \quad 19 \%$ nuclear

\section{NUCLFAR POWER}

Policy: Strong support for domestic use and export of the CANDU reactor system.

$\begin{array}{llll}\text { Nuclear Power Capacity } & 1992 & 14.1 & \text { GWe } \\ & 1995 & 15.5 & \text { GWe } \\ & 2000 & 15.5 & \text { GWe }\end{array}$

$\begin{array}{llll}\text { Reactor Mix } & 1993 & \text { PHWR } & 22(1971-93)\end{array}$

\section{INDUSTRIAL FUEL CYCLE}

Policy: Interim storage of used fuel for decades, pending an environmental assessment and review of a concept for the disposal of nuclear fuel waste (review process of concept has started).

Waste Management Strategy: Geologic disposal of nuclear fuel waste and spent CANDU fuel in a crystalline rock repository. Disposal of LLW in engineered, shallow-ground facility. 
Cumulative Used Fuel (PHWR) $1991 \quad 15,877$ IU $2000 \quad 17,271 \mathrm{tU}$

\section{Major Milestone}

- Public hearings by review panel on Nuclear Fuel Waste Management Disposal Concept (AECL-prepared EIS)

\section{INTERNATIONAL RELATIONSHIPS}

DOE/AECL Agreement for Cooperation in Radioactive Waste Management

Term: $\quad 09-08-76$ to $09-29-96$

Scope: Waste treatment, storage, geological disposal, transportation requirements, operational considerations; environment and safety; public acceptance issues; information exchange in radioactive waste management, geological disposal, waste form characterization, waste/used fuel storage, and intercomparison of performance assessment computer models and codes.

\section{DOE/AECL Subsidlary Agreement \# 2}

Term: $09-30-91$ to 09-29-96

Scope: Joint project to study the preparation/packaging of radioactive wastes/disposal in geologic formation; fundamental materials investigations; in-situ stress determination; SF dissolution model development; large block tracer test; laboratory and field tests of in-situ hydrochemical tool; Cigar Lake analogue study; actinide/fission product geochemistry; performance assessment technology exchange; multiple-well hydraulic test and field tracer test development.

Member of LAEA and OECD/NEA - Exchange agreements with the following agencies and countries: DOE/U.S.; SKB/Sweden; UKAEAU.K; PNC, JAERI/Japan; KAERI/Korea; TVO, IVO/Finland; CEA, ANDRA/France; ONDRAF/Belgium; ENRESA/ Spain. 


\section{ORGANIZATION}

- AECB (Atomic Energy Control Board)--federal nuclear control agency, answers to Parliament, responsible for health/safety regulation, compliance/licensing.

- AECL (Atomic Energy of Canada Limited)--Crown Corporation, answers to Parliament via Ministry of Natural Resources. R\&D; design, engineering and sale of CANDU and research reactors; proprietary rights on CANDU Nuclear Steam Supply Systems; waste management $R \& D$ at Whiteshell and Chalk River laboratories.

- OH (Ontario Hydro)--provincial public utility. Owns/operates 20 CANDU nuclear power plants, 15,340 MWe total capacity; responsible for developing interim fuel storage/transport technologies.

- HQ (Hydro Quebec)--provincial public utility. Owns/operates Gentilly 2, a 600-MWe CANDU station.

- NBEPC (New Brunswick Electric Power Commission)--provincial public utility. Owns/operates Point Lepreau Nuclear Generating Station, a 600-MWe CANDU. 


\section{FEDERAL GOVERNMENT RESPONSIBILITIES--FUEL}

\section{CYCLE/WASTE MANAGEMENT}

Ministry of Natural Resources Canada

Alomic Energy Control Board (AECB)

- Regulations, Licensing, Compliance

- Atomic Energy of Canada, Limiled (AECL)

- AECL CANDU

- Reactor Design, Engineering Export, proprietary rights on CANDU Nuclear Steam Supply Systems

- AFCL Research (see CA-5)

- Natural Resources Canada

- Geological Survey of Canada (GSC)

- Information/Services Branch

- Minerals/Continental Geoscience Branch

- Sedimentary/Cordilleran Geoscience Branch

- Geophysics/Marine Science Branch

- Mineral/Energy Technology (MET)

1

Canadian Centre for Mineral/Energy Technology (CANMET)

- Mining Research Laboratories

- Sudbury Laboratory

- Elliot Lake Laboratory

- Canadian Mining Technology Laboratory

- Mineral Sciences Lahoratories

- Radionuclide Recovery from Thorium Mill Tailings

- Metals Technology Laboratorles 
ATOMIC ENERGY OF CANADA LIMITED-WASTE MANAGEMENT ORGANIZATION

\section{AECL RESEARCH}

Whiteshell Laboratories (WL)

- Environmental Sciences Waste Management Program

- Disposal Technology

- Applied Geoscience

- Environmental/Safety Assessment

- Geotechnical Science/Engineering

- Underground Research Laboratory

- Environmental Technology

- Environmental Science

- Geochemistry Research

- Puel Waste Technology

Chalk River Laboratories (CRL)

- Waste Management Systems

- Storage/Disposal

- Waste Processing Technology

- Health Sciences

- Radiation Biology

- Dosimetry Research

- Environmental Research

Head Onice, Ottawa

- Low-Level Radioactive Waste Management Ofice (operated by AECL Research on behalf of Natural Resources) 


\section{AECB}

Atomic Energy Control Board

P.O. Box 1046

270 Albert Street

Ottawa, ON

K1P 5 S9 Canada

Tel: 613-995-5894

Fax: 613-995-5086

President

Director General,

Fuel Cycle/Materials Regulations

Wastes/Impacts Div.

Compliance Serv. /Laboratory Div.

Radioisotopes/Transportation Div.

Uranium Facilities Div.

Director, Research/Safeguards

Safeguards/Security Div.

Director, Analysis/Assessment

Safety Evaluation (Analysis) Div.

Safety Evaluation (Engineering) Div.

Dr. René J. A. Levesque

Murray Duncan

George C. Jack

Cait Maloney

Ross Brown

Tom Viglasky

Jim Harvie

John R. Coady

John Waddington

Peter Wigfull

Kurt Asmis

AECL

Atomic Energy of Canada Ltd.

344 Slater Street

Ottawa, ON

Tel: $\quad 613-237-3270$

K1A OS4 Canada

Fax: $\quad 613-563-9499$

Chairman

President

President, AECL Research

Agnes Bishop

Director, LLW Management

Dr. Terry E. Rummery

Dr. Robert W. Pollock 


\section{AECL-CRL}

AECL Research

Chalk River Laboratories

Chalk River, ON

Tel: $\quad 613-584-3311$

ROJ 1 JO Canada

Fax: 613-589-4024

Health Sciences

Dr. Richard V. Osborne

Radiation Biology

Dr. Norm E. Gentner

Waste Management Systems

Dr. Don J. Cameron

Public Affairs, Director

John Macpherson

\section{Facilities}

- WTC (Waste Treatment Center)

Mission: Development and operation of processes for the treatment of LLW and ILW using incineration, compaction, microfiltration/reverse osmosis evaporation, ion exchange, and solidification in bitumen.

- IRUS (Intrusion Resistant Underground Structure) Mission: LLW/LW repository consisting of prototype vault. Capacity of $2,000 \mathrm{~m}^{3}$ radwaste in drums or bales, when full to be covered with backfill, roofed with concrete, and mounded with earth. Waste can be retrieved from the IRUS vauit until concrete cap is emplaced. Construction start, 1993. 
AFCL-WL

AECL Research

Whiteshell Laboratories

Pinawa, MB

ROE 1 LO Canada

Underground Research Laboratory

V.P., Environmental Sciences/WM

Disposal Technology

Applied Geoscience

Environmental/Safety Assessment

Geotech. Science/Engineering

URL, Mgr.

Environmental Technology

Environmental Science

Geochemistry Research

Fuel Waste Technology

Environmental Review Ofice

Government/Public Affairs

Tel: $\quad 204-753-2311$

Fax: 204-753-8404

Tel: $\quad 204-345-8625$

Fax: $\quad 204-345-8868$

Dr. Collin J. Allan

Dr. Ken W. Dormuth

Cliff Davison

Dr. Alf Wikjord

Gary Simmons

Mitch Ohta

Dr. Keith Nuttal

Dr. Reto Zach

Dr. Peter Sargent

Lawrence Johnson

Barbara Gray

Mary Greber

\section{Facilities}

- BITF (Borehole Instrumentation Test Facility)

Mission: Test and calibrate geotechnical borehole instruments under pressure, temperature, and chemical conditions that could exist in exploration boreholes to depths of $1200 \mathrm{~m}$ below ground surface in granitic rock.

Design Basis: Stainless steel vertical test chamber to simulate a 10-m-long borehole section, 76-mm inside diameter. Temperature, pressure, flow rates, and water chemistry can be precisely controlled and monitored.

History: Startup, 1983.

(contd next page) 


\section{$\triangle 1$ ECL-WL (contd)}

- URL (Underground Research Laboratory), located about $20 \mathrm{~km}$ northeast of WL in the Lac du Bonnet granite batholith. Mission: Operate facility ensuring compliance with regulatory standards and high level of safety/quality control; design and implement in-situ and laboratory experiments/engineering demonstrations to investigate relevant issues.

Design Basis: Access shaft and ventilation raised to 430-m depth with shaft stations at $130-\mathrm{m}, 240-\mathrm{m}, 300-\mathrm{m}$, and 420 -m depths. Licensed radioactive sources and tracers may be used, but no radioactive wastes can be employed. A series of nine experiments is in progress on the 240 - and $420-\mathrm{m}$ levels and in the surrounding rock mass.

Hislory: Underground access development complete in 1990; major experiments in solute transport, rock mass response, characterization method, and engineered sealing systems began in 1988.

- IFTF (Immobilized Fuel Test Facility)

Mission: Test the effects of water, heat, and pressure on used fuels, container materials, buffer, and rock in the presence of a radiation field.

Design Basls: A high-level radiation source is used in heated concrete canisters to provide a gamma radiation source. Pressure vessels adjacent to the source contain the material to be studied. Adjacent "warm cells" are available for experiments involving moderate levels of radiation.

History: First canister loaded, 8/1984.

(contd next page) 


\section{AECL-WL (contd)}

- LBRMF (Large Block Radionuclide Migration Facility) Mission: Study the migration of reactive and nonreactive contaminants, including radionuclides, over a distance up to $1 \mathrm{~m}$ through natural fractures in quarried, intact rock; determine the spatial distribution of sorbed radionuclides on fracture surfaces and in the rock matrix at the end of the migration experiments. Design Basis: The facility consists of an experimental section, equipped with moveable active fume hoods to hold quarried rock; an analysis section, equipped with a 2-D gamma scanner, active fume hoods, and equipment to handle blocks of rock up to $2000 \mathrm{~kg}$.

History: Joint migration experiment with U.S. DOE using uranine (tracer dye), ${ }^{131} \mathrm{I}$, and ${ }^{137} \mathrm{Cs}$ has been completed and results published. Second experiment, using uranine, ${ }^{3} \mathrm{H},{ }^{85} \mathrm{Sr}$, ${ }^{95 \mathrm{~m}} \mathrm{Tc}$, ${ }^{157} \mathrm{Cs}$, and ${ }^{144} \mathrm{Ce}$ has been completed; third experiment, using ${ }^{85} \mathrm{Sr},{ }^{131} \mathrm{I},{ }^{137} \mathrm{Cs},{ }^{144} \mathrm{Ce},{ }^{152} \mathrm{Eu},{ }^{231} \mathrm{~Np}$ and ${ }^{238} \mathrm{Pu}$ has been carried out for PNC, Japan; colloid migration experiments are under way; migration experiments in fractures with simple, uniform geometrics are planned in cooperation with LANL (U.S).

\section{NATURAL RESOURCES CANADA}

Natural Resources Canada

Science and Technology

580 Booth Street

Ottawa, ON

Tel: $\quad 613-995-1240$

K1A OE4 Canada

Fax: 613-996-9094

Electricity Branch, Dir. General $\quad$ Dr. R. W. Morrison

613-992-3370

Radioactive Waste/Radiation, Mgr. Dr. Peter Brown

613-996-2395 


\section{NATURAL RESOURCES CANADA-CANMET}

Natural Resources

Canada Centre for Mineral and Energy Technology

555 Booth Street

Ottawa, ON

Tel: $\quad$ 613-995-4194

K1A 0G1 Canada

Fax: 613-996-9673

Policy Planning/Services, Dir. Gen. J. Ferron

Mineral Technology (A)

Waste Mgmt., Elliot Lake Irwin Itzkovitch

Mining Research Laboratory

T. Mottahed

J. E. Udd

\section{NATURAL RESOURCES CANADA-GSC}

Natural Resources

Geological Survey of Canada

601 Booth Street

Ottawa, ON

K1A 0E8 Canada

Tel: $\quad 613-992-5910$

Fax: 613-995-3082

Assistant Deputy Minister

Dr. E. A. Babcock

Chief Scientist

Dr. Jim Franklin 
$\underline{\text { OH}}$

Ontario Hydro

700 University Avenue

Toronto, ON

Tel: $\quad 416-592-5111$

M5G 1 X6 Canada

Fax: $\quad 416-592-4485$

Nuclear Fuel Supply

Design/Development Div., Director

Nuclear Engineering

Radioactive Mtls. Management

Rad. Waste Storage/Disposal

Rad. Mlts. Transportation

SF Management/Decommissioning

Fuel Cycle, Isotope

Fax. 416-592-4485

E. G. Bazeley

Hugh S. Irvine

Don W. Souther

Dr. Peter Stevens-Guille

P. J. Armstrong

Joe F. Tanaka

Syed J. Naqvi

R. A. McEachran

RWOS (Radioactive Waste Operations Site)

Bruce Nuclear Power Development

Box 1540

Tiverton, $\mathrm{ON}$

Tel: $\quad 519-361-2673$

NOG 2T0 Canada

Waste Operations

Keith Mombourquette

Tel: $\quad$ ext, -4844

Fax: $\quad 519-361-4345$

Function: Process/store low- and intermediate-level radioactive waste from Ontario Hydro CANDU reactors and research/ maintenance facilities.

Facilities

- WVRF (Waste Volume Reduction Facility)

Processing Equipment: Two-chamber pyrolysis incinerator with a capacity of $30 \mathrm{~kg} / \mathrm{h}$; box compactor with a compaction force of $2 \times 10^{6} \mathrm{~N}$ low force drum crusher. Startup, 1977 (box compactor replaced baler in 1993).

(contd next page) 


\section{$\underline{\text { OH}}$ (contd)}

- LLW Storage Facilities

- 5 above-ground warehouse-type bulldings; waste with a radiation field of $<1 \mathrm{R} / \mathrm{h}$ at $30 \mathrm{~cm}$ is stored in stackable containers with a storage capacity of $8000 \mathrm{~m}^{3}$ each.

- 15 trenches; reinforced concrete structures $-3 \mathrm{~m}$ below ground; designed for waste with radiation fields $>1 \mathrm{R} / \mathrm{h}$ but $<15$ $\mathrm{R} / \mathrm{h}$; storage capacity ranges from 360 to $680 \mathrm{~m}^{3}$ each.

- 15 quadricells; above-ground, reinforced concrete structures; sufficient shielding for storage of waste with radiation fields of $>15 \mathrm{R} / \mathrm{h}$, e.g., ion exchange resins, filters, and reactor core components; storage capacity ranges from 1 to $18 \mathrm{~m}^{3}$.

- 358 in-ground containers; welded steel liners concreted into augered holes; designed for storage of waste with radiation fields $>15 \mathrm{R} / \mathrm{h}$, e.g., ion exchange resins, filters and reactor core components. In-ground storage consists of $2241 \mathrm{~m}^{3}$ capacity tile holes and 134 in-ground containers. In-ground container capacity ranges from 2 to $18 \mathrm{~m}^{3}$ ( 54 were added in 1993).

- Paved area $4700 \mathrm{~m}^{2}$ for storage of contaminated tooling in 6.1-m-long marine cargo containers stacked two high.

- 27 in-ground contalners with varying diameters/depths containing scrap heat exchangers; augered holes with crushed limestone backfill. Storage capacity ranges from 2 to $18 \mathrm{~m}^{3}$ each. 


\section{CHINA}

\section{(People's Republic of China)}

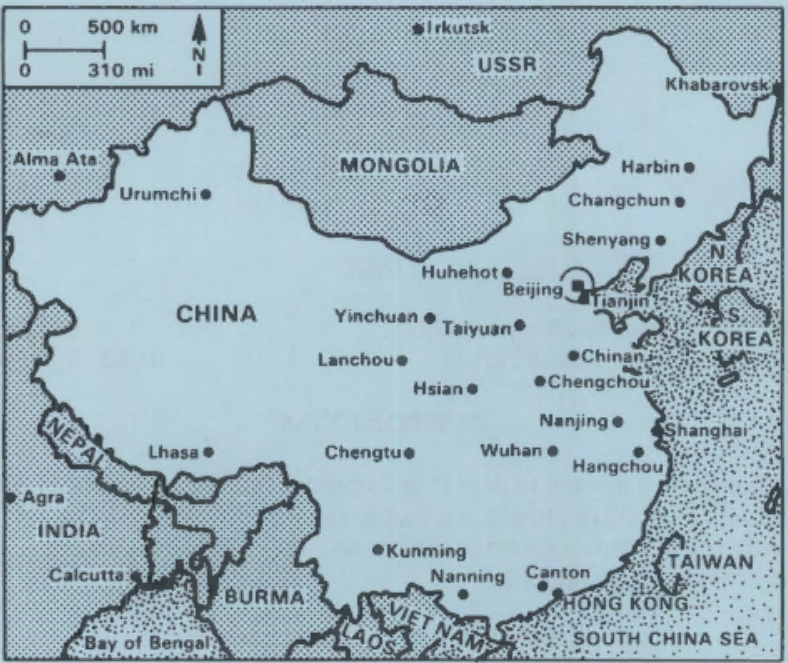




\section{CHINA}

MAJOR PUBLIC HOLIDAYS (1994)

$\begin{array}{lrll}\text { Jan } & 1-2 & \text { New Year } \\ \text { Feb } & 10-11 & \text { Spring Festival } \\ \text { Mar } & 8 & \text { Women's Day } \\ \text { May } & 1 & \text { Labor Day } \\ \text { Jun } & 1 & \text { Children's Day } \\ \text { Oct } & 1-2 & \text { National Day }\end{array}$

TIME

Standard Time Washington, D.C.

+13 hours

Daylight Savings Time Period:

$04 / 03-09 / 03 / 94$

\section{PASSPORT/VISA}

A passport is needed to depart and re-enter the U.S.; in addition, a visa is currently required for a visit to the People's Republic of China. Most travel agencies can provide up-to-date information concerning requirements.

\section{CURRENCY EXCHANGE RATE}

1 U.S. $\$=8.7000$ Renminbi

per Wall Street Journal, 01/31/94. As rates fluctuate daily, it is recommended to obtain current rates from local banks or newspapers prior to departure.

\section{U.S. EMBASSY - BEIJING}

American Embassy

Xiu Shui Bei Jie 3

Beijing 100600

Tel: $\quad 86-1-532-3831$

People's Republic of China

Fax: $\quad 86-1-532-3178$

Science Counselor

Marco S. DiCapua 
Population

1993

1.18 billion

\section{ENERGY}

Electric Power Capacity

$\begin{array}{rrl}1993 & 180 & \text { GWe } \\ & <1 \% & \text { nuclear } \\ 1995 & 206 & \text { GWe } \\ & <1 \% & \text { nuclear } \\ 2000 & 285 & \text { GWe } \\ & <1 \% & \text { nuclear }\end{array}$

Electric Power Production

$\begin{array}{rrl}1993 & 815 & \text { TWh } \\ & 74 \% & \text { coal } \\ & 18 \% & \text { hydro } \\ & 8 \% & \text { oil } \\ & <1 \% & \text { nuclear } \\ 2000 & <1 \% & \text { nuclear }\end{array}$

\section{NUCLEAR POWER}

Policy: Develop nuclear power as one of three major sources of energy to solve problems caused by uneven distribution of resources; be self-sufficient, but introduce foreign advanced technology.

Nuclear Power Plant Capacity

$\begin{array}{lll}1993 & 0.3 & \text { GWe } \\ 1995 & 2.1 & \text { GWe } \\ 2000 & 6.0 & \text { GWe }\end{array}$

Reactor Mix

1993 PWR 1 (1993)

PWR 2 (1994)

Reactor Development PWR, HTGR, FBR, LTR

\section{INDUSTRIAL FUEL CYCLE}

Policy: Activities include uranium mining, milling, and diffusion enrichment; isotope separation; fuel fabrication; future spent fuel reprocessing.

Waste Management Strategy: Interim storage of spent fuel in pools for 5-8 years if $<1,000 \mathrm{tU}$, in transport/storage casks if $>1,000 \mathrm{tU}$; interim storage, reprocessing, vitrification, and disposal all to be at 
one site, to be selected, located in northwest China or the Gobi Desert; final disposal in deep geologic formation; plan for a small pilot reprocessing plant, followed by a commercial-size facility, about $500 \mathrm{tU} / \mathrm{a}$.

\section{INTERNATIONAL REIAATIONSHIPS}

Member of LAEA. Cooperative agreements have been signed with Argentina, Canada, France, Germany, Italy, Japan, and the U.S. (nuclear safety).

\section{ORGANIZATION}

- CNNC (China National Nuclear Corporation) -- fuel cycle development

- CIAE (China Institute of Atomic Energy)

- CNEC (China Nuclear Engineering Corporation) -- handles import and export

- China Zhongyuan Engineering Corporation --provides technical services and engineering work, contracts building projects.

- Southwest Institute of Physies -- nuclear R\&D.

- NNSA (National Nuclear Safery Administration) -- responsible for standards/regulations, construction permits/operating licenses, monitoring plant operations; conducts joint safety research with other nations.

- INET (Institute of Nuclear Energy Technology), Tsinghua University

\section{BINE}

Beijing Institute of

Nuclear Engineering

P.O. Box 840

Tel: $\quad 86-1-842-3311$

Beijing 100840, PRC

Fax: 86-1-841-5067

Director

Director, Nuclear Waste Mgmt.

Director, International Affairs

Lin Guoming

Sun Donghui

Liu Dinggin 


\section{CIAE}

China Institute of

Atomic Energy

P.O. Box 275

Beijing 102413, PRC

Tel: $\quad 86-1-935-7676$

Fax: $\quad 86-1-935-7008$

Honorary Director

Director

Director, Radiochemistry

Wang Chanchang

Sun Zuxun

Luo Shanggeng

Function: Large comprehensive nuclear R\&D institute. FBR development.

Waste Management R\&D: HLW vitrification; waste form characterization; pilot plants to be built.

\section{CNEIC}

China Nuclear Energy Industry Corporation

P.O. Box 822

Beijing 100037, PRC

Tel: $\quad 86-1-851-2211$

Fax: 86-1-851-2393

General Manager

Zhou Yuanquan

Function: Import/export company for the nnclear industry. 


\section{CNNC}

China National Nuclear Corporation P.O. Box 2102

Beijing 100822, PRC

Tel: $\quad 86-1-851-2211$

Fax: $86-1-851-2393$

President

Nuclear Fuel Department

Jiang Xingxiong

Nuclear Radiation Protection, Wang Xiaoli

Environment/Health Department

Zhu Zhiming

Conglomerate of over 200 enterprises and institutions. Plans to construct four regional final LLW/ILW disposal facilities in northwest (Gansu), east, south, and southwest China for waste from nuclear facilities, including Qinshan and Daya Bay nuclear power stations.

\section{INET}

Institute of Nuclear Energy Technology Tsinghua University

P.O. Box 1021

Beijing 102201, PRC

Tel: $\quad 86-1-259-4533$

Fax: 86-1-256-4177

Director

Dep. Director

Academic Committee Director

Prof. Wu Zongrin

Prof. Xu Yuanhui

Prof. Zhu Yongjun

Designed/built low-temperature reactor ( $5 \mathrm{MWth}$ ), which provides central heating for the institute's off-campus research facility.

$\underline{\text { NFC }}$

Nuclear Fuel Complex

P.O. Box 508

Lanzhou 732850, PRC

Tel: $\quad 86-0931-841-7584$

Fax:

Director

Liu Qizhao 


\section{NFF}

Nuclear Fuel Fabrication

P.O. Box 257

Tel: $\quad 86-0831-22-1811$

Chengdu, Yibin 610002

Fax: $\quad 86-0831-22-3622$

Function: Production of fuel for the $300 \mathrm{MWe}$ PWR being built at Quinshan (near Shanghai) and the two $900 \mathrm{MWe}$ PWRs at Daya Bay (near Hong Kong).

\section{NNSA}

National Nuclear Safety

Administration

P.O. Box 8088

Tel: $\quad 86-1-225-8583$

Beijing 100088, PRC

Fax: 86-1-225-7804

Director General

Huang Qitao

Chief Engineer

Dep. Chief Engineer

Li Zhiyu

Zhang Chonguan

Function: Responsible for standards/regulations, construction permits/operating licenses; monitoring plant operations; joint safety research with other nations. Cooperation with U.S. (NRC)

\section{NPIC}

Nuclear Power Institute of China

P.O. Box 430

Chengdu 610041, PRC

Tel: $\quad 86-028-558-2199$

Fax: $\quad 86-028-558-2223$

Director

Zhao Chengknn

Function: Designing Qinshan II (600 MWe PWR); R\&D on advanced PWRs. 


\section{SNERDI}

Shanghai Nuclear Engineering and Design Institute 29 Hongchao Road Tel: $\quad 86-021-436-4700$ Shanghai 200233, PRc Fax: $\quad 86-021-439-0846$

Director

Foreign Affairs Gen Gen Quchang $\mathrm{Du} \mathrm{Li}$

Function: Designing (300 MWe) PWRs for export; designed Qinshan I. 


\section{FINLAND}

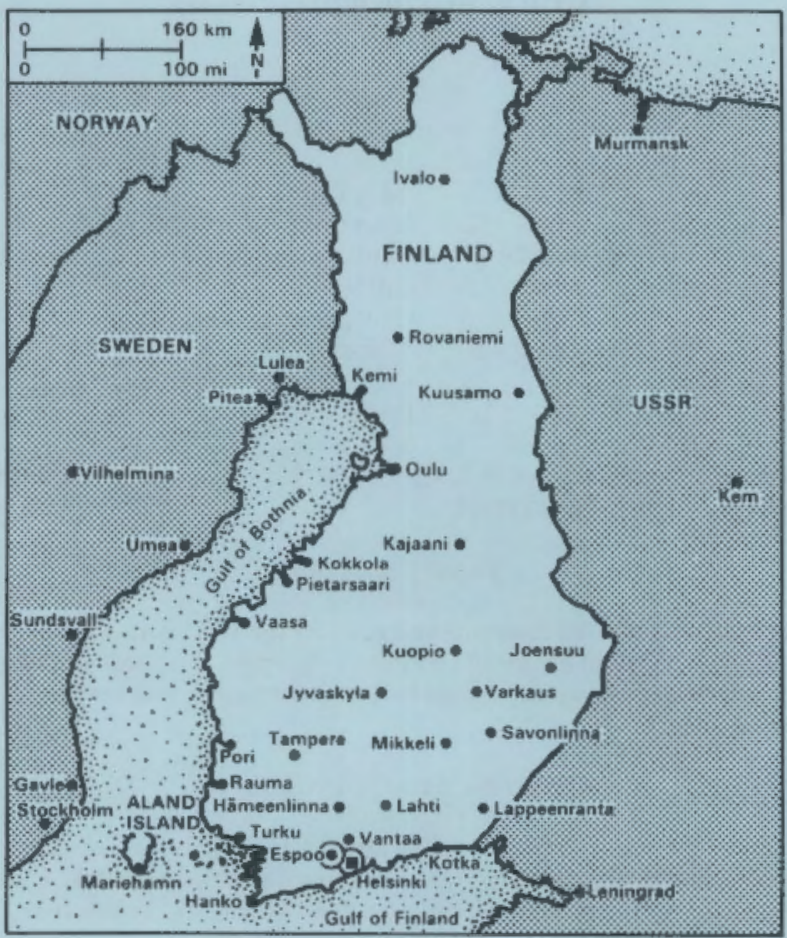




\section{FINLAND}

\section{MAJOR PUBLIC HOLIDAYS (1994)}

$\begin{array}{lrl}\text { Jan } & 1 & \text { New Year } \\ \text { Jan } & 6 & \text { Epiphany } \\ \text { Apr } & 1 & \text { Good Friday } \\ \text { Apr } & 3-4 & \text { Easter } \\ \text { May } & 1 & \text { May Day } \\ \text { May } & 12 & \text { Ascension } \\ \text { Jun } 24-25 & \text { Midsummer Eve/Day } \\ \text { Nov } & 5 & \text { All Saints } \\ \text { Dec } & 6 & \text { Independence Day } \\ \text { Dec } 24-26 & \text { Christmas }\end{array}$

\section{TIME}

Standard Time Washington, D.C.

\section{PASSPORT/VISA}

A passport is needed to depart and re-enter the U.S. A visa is cnrrently not required for a visit to Finland; however, it is recommended to consult a travel agency for up-to-date information concerning requirements.

\section{CURRENCY EXCHANGE RATE}

$$
1 \text { U.S. } S=5.50 \text { Markka (FIM) }
$$

per Wail Street Jonrnal, 01/31/94. As rates fluctuate daily, it is recommended to obtain current rates from local banks or newspapers prior to departure.

\section{DIRECT DIALING}

Individual numbers for direct-dial to Finland are complete as listed, after dialing international access code: 011 . Country code is 358; listed local nnmbers include city code.

\section{U.S. EMBASSY - HELSINKI}

American Embassy

\section{Itaeinen Puistotie 14A 00140 Helsinki Finland}

Economic Section
Tel: $\quad 358-0-17-1931$

Fax: $\quad 358-0-65-6846$

Robert W. Boehme 
Population

1992

5 million

\section{ENERGY}

$\begin{array}{lcrl}\text { Electric Power Capacity } & 1992 & 12.6 & \text { GWe } \\ & 1995 & 18 \% & \text { nuclear } \\ & & 17 \% & \text { GWe } \\ \text { nuclear } \\ & 2000 & 15.5 & \text { GWe } \\ & & 16 \% & \text { nuclear } \\ \text { Electric Power Production } & 1992 & 57.4 & \text { TWh } \\ & & 34 \% & \text { nuclear } \\ & & 26 \% & \text { hydro } \\ & & 15 \% & \text { solids } \\ & & 14 \% & \text { coal } \\ & & 2 \% & \text { gas } \\ & & 29 \% & \text { oil } \\ & & & \text { nuclear } \\ & 1995 & 23 \% & \text { nuclear }\end{array}$

NUCLEAR POWER

$\begin{array}{llrl}\text { Nuclear Power Capacity } & 1992 & 2.3 & \text { GWe } \\ & 2000 & 2.3 & \text { GWe } \\ \text { Reactor Mix } & 1993 & \text { PWR } & 2(1977 / 81) \\ \text { BWR } & 2(1979 / 82)\end{array}$

\section{INDUSTRIAL FUEL CYCLE}

Pollcy: Purchase fuel and fuel-cycle services from other countries (spent fuel from Soviet-built reactors is returned to Russia).

Waste Management Stralegy: Spent fuel from TVO's power plants will be stored for 40 years, then disposed of in crystalline bedrock; IVO returns spent fuel from its (Soviet-built) reactors to Russia. Operating wastes are conditioned, stored above ground, and disposed of in crystalline bedrock at the nuclear power station sites. Decommissioning wastes will be disposed of in extended operating waste repositories. 
Cumulative SF Arisings (LWR), tU removed + cores

$$
\begin{array}{ccc} 
& \text { TVO } & \text { IVO } \\
1990 & 450+177 & 330+75 \\
2000 & 855+177 & 580+75
\end{array}
$$

\section{Major Milestones}

- Complete LLW/LWW repository (IVO)

- Complete SF repository site selection (TVO) 2000

- Complete SF repository (TVO)

\section{INTERNATIONAL RELATIONSHIPS}

Member of IAEA and OECD/NEA; collaboration with Sweden, Canada, Denmark, Norway, and Switzerland in waste management studies. Purchase of fuel-cycle services: uranium from Canada, Australia, Niger, China, and Russia; uranium conversion/enrichment from Canada, France, Germany, Russia, and U.K.; fuel element fabrication from Sweden, Germany, and Russia; return of spent fuel to Russia (IVO).

\section{ORGANIZATION}

- Council of State (Cabinet of Ministers)--grants licenses.

- Nuclear Energy Commission--advisory organization for matters connected with the use of nuclear energy.

- Advisory Committee on Nuclear Safety--advisory organization.

- KTM (Ministry of Trade and Industry)--Energy Department, formulates energy policies, grants licenses.

- STUK (Finnish Centre for Radiation and Nuclear Safety)--regulatory authority which also conducts research, particularly related to transport of radionuclides in biosphere.

- IVO (government-owned power company)--operates two Soviet-built PWRs.

- TVO (power company)--operates two Swedish-built BWRs. 
- VTT (Technical Research Centre of Finland)--nuclear research, inclnding waste management $R \& D$.

- Geological Survey of Finland--bedrock-related research.

- University of Helsinld--basic research on radiochemistry.

- Helsinkd University of Technology--basic research.

\section{ADVISORY COMMITTEE ON NUCLEAR SAFETY}

Advisory Committee on Nuclear Safety

P.O. Box 14

Laippate 4

FIN-00881 Helsinki

Finland

Tel: $\quad 358-0-708-21$

Fax: $\quad 358-0-708-2392$

Chairman

Secretary-General

Prof. Jarl Forstén

Hannu Koponen

Function: Advisory organization for safety matters connected with the nse of nnclear energy; coordinated by the Finnish Centre for Radiation and Nuclear Safety (STUK).

\section{GEOLOGICAL SURVEY OF FINLAND}

Geological Survey of Finland

Betonimiehenkuja 4

Tel: $\quad 358-0-469-31$

FIN-02150 Espoo, Finland

Fax: $\quad 358-0-462-205$

Director

Nuclear Waste Disposal

Prof. Veikko Lappalainen

Paavo Vnorela

R\&D Activitles: Geological studies for waste disposal in crystalline bedrock. 


\section{IVO (National Power Company)}

Imatran Voima Oy

Rajatorpantie 8

Tel: $\quad 358-0-508-1$

FIN-01019 IVO, Finland

Fax: $\quad 358-0-563-6823$

President, CEO

Dr. Kalevi Numminen

Exec. VP, Generation

Dr. Anders Palmgren

Director, Research

Nuclear Waste Prof. Pekka Salminen Jussi Paimu

Function: Operate two nuclear power plants (Soviet built) at Loviisa, southeastern Finland.

Owner: Government.

$\underline{\text { NEC }}$

Nuclear Energy Commission

Pohjoinen Makasiinikatu 6

FIN-00130 Helsinki

Tel: $\quad 358-0-160-5222$

Finland

Fax: $\quad 358-0-160-2695$

Chairman

Secretary-General

Prof. Pekka Silvennoinen

Sakari Immonen

Function: Advisory organization for general matters connected with nuclear energy; coordinated by the Ministry of Trade and Industry. 


\section{STUK}

Finnish Centre for Radiation and Nuclear Safety

P.O. Box 14

Laippatie 4

Tel: $\quad 358-0-7082-1$

FIN-00881 Helsinki, Finland

Fax: $\quad 358-0-7082-392$

Director

Nuclear Safety Department

Prof. Antti Vuorinen

Nuclear Fuel Cycle

Jukka Laaksonen

Nuclear Waste Tero Varjoranta Esko Ruokola

Function: Regulatory enforcement and inspection authority; research related to transport of radionuclides in biosphere.

\section{TVO (Industrial Power Company)}

Teollisuuden Voima Oy

Annankatu 42C

Tel: $\quad 358-0-6180-1$

FIN-00100 Helsinki, Finiand

Fax: $\quad 358-0-6180-2570$

Director

Director, Development

Mauno Paavola

Nuclear Fuel

Ami Rastas

Nuclear Waste

Ilkka Mikkola

Veijo Ryhänen

Function: Operate two nuclear power units (Swedish-built BWRs) at Olkiluoto in Eurajoki, southwestern Finland; manage fuel procurement and TVO-produced wastes.

Owners: $57 \%$ private, $43 \%$ public utilities/government-owned companies.

(contd next page) 


$$
\text { TVO (contd) }
$$

\section{Facilities}

- KPA-STORE (Interim storage facility for spent nuclear fuel) located at reactor site; three pools with total capacity of $1200 \mathrm{tU}$; construction completed 1987.

- VLJ Repository (Final repository for TVO's ILW/LLW) - located at reactor site; LLW and ILW packaged in metal drums/containers are buried in two silos 70-100 m deep; ILW silo has reinforced 0.6m thick concrete liner, capacity $8400 \mathrm{~m}^{3}$; construction completed $12 / 1991$.

\section{VTT (Technical Research Centre of Finland)}

\section{VTT Energy}

Nuclear Energy

Tekniikantie 4C, Espoo

FIN-2044 VTT, Finland

Tel: $\quad 358-0-456-1$

Fax: $\quad 358-0-456-5000$

Nuclear Energy Research

Reactor Physics

Nuclear Waste Management

Prof. Lasse Mattila

Ms. Riitta Kurki-Rajamäki

Dr. Seppo Vuori

R\&D Activities: Reactor physics, nuclear power plant safety analyses; safery analysis of final disposal of wastes in bedrock, including groundwater flow modeling.

VTT Chemical Technology Environmental Technology

Physics Bldg.

Otakaari 3A, Espoo

FIN-02044 VTT, Finland

Tel: $\quad 358-0-456-1$

Fax: $\quad 358-0-456-6390$

Head

Nuclear Waste

Dr. Kari Larjava

Arto Muurinen

(contd next page) 
VTT (contd)

R\&D Activities: Dissolution of spent fuel and actinides; geochemical modeling; migration in barriers and bedrock; characterization of ILW forms.

VTT Communities/Infrastructure

Rock/Environmental Engineering

Betonimiehenkuja 1, Espoo

P.O. Box 19041

Tel: $\quad 358-0-456-1$

FIN-02044 VTT, Finland

Fax: $\quad 358-0-467-7927$

Head

Waste Disposal

Jukka Pöllä

Petteri Pitkänen

R\&D Activities: Hydrogeological and geomechanical measurements, characterization and performance of bedrock and engineered barriers.

VTT Manufacturing Technology

Materials/Structural Integrity

Kemistintie 3, Espoo

P.O. Box 1704

Tel: $\quad 358-0-456-1$

FIN-02044 VTT, Finland

Fax: $\quad 358-0-456-7002$

Head

Nuc. Materials Research

Dr. Kari Törrönen

Periti Aaltonen

R\&D Activities: Corrosion of encapsulation materials in repository conditions. 


\section{UNIVERSITY OF HELSINKI}

University of Helsinki

Department of Radiochemistry

P.O. Box 5, Unioninkatu 35

FIN-00014 UNIVERSITY OF HELSINKI

Finland

Tel: $\quad 358-0-191-1$

Fax: $\quad 358-0-191-7814$

Director

Prof. Timo Jaakkola

R\&D Activities: Waste treatment processes, migration of radionuclides, and radiochemical analysis.

\section{HELSINKI UNIVERSITY OF TECHNOLOGY}

Nuclear Engineering Laboratory

Rakentajaaukio 2C

Tel: $\quad 358-0-451-1$

FIN-02150 Espoo, Finland

Fax: $\quad 358-0-451-4068$

Nuclear Energy/

Advanced Energy Systems

Prof. Rainer Salomaa

Tel: $\quad 358-0-451-3199$

Fax: $\quad 358-0-451-3195$ 


\section{FRANCE}

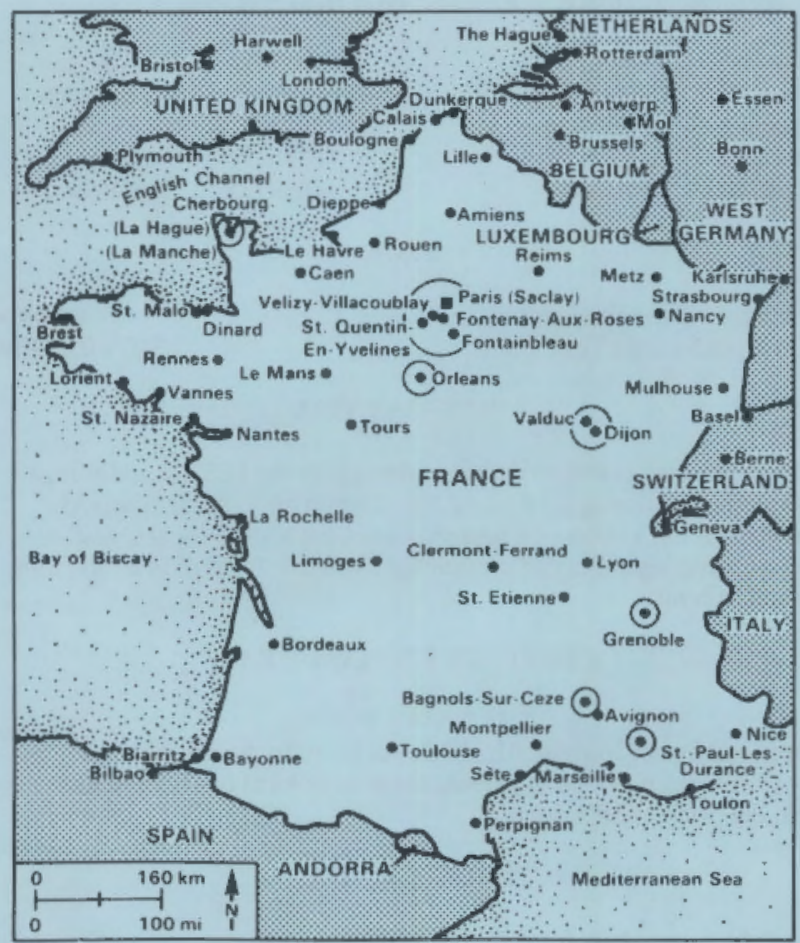


FRANCE

MAJOR PUBLIC HOLIDAYS (1994)

Jan 1 New Year

Apr 4 Easter Monday

May 1 Labor Day

May 12 Ascension

Jun 23 Pentecost
Jul 14 Bastille Day

Aug 15 Assumption

Nov 1 All Saints

Nov 11 Veterans Day

Dec 25 Christmas

TIME

Standard Time Washington, D.C. +6 hours Daylight Savings Time Period:

$03 / 27-09 / 24 / 94$

\section{PASSPORT/VISA}

A passport is needed to depart and re-enter the U.S.; in addition, a visa is currently required when traveling on an 'official' passport to France but not when a personal passport is used for the visit. Most travel agencies can provide up-to-date information concerning requirements.

\section{CURRENCY EXCHANGE RATE}

1 U.S. $\$=5.88$ Franc

per Wall Street Jonrnal, 01/31/92. As rates fluctuate daily, it is recommended to obtain current rates from local banks or newspapers prior to departure.

\section{DIRECT DIALING}

Individual numbers for direct dial to France are complete as listed, after dialing international access code: $\mathbf{0 1 1}$. Conntry code is 33; listed local numbers include city code.

\section{U.S. EMBASSY - PARIS}

American Embassy

2 Avenue Gabriel

75382 Paris

France

Tel: $\quad 33-1-42-96-12-02$

Fax: $\quad 33-1-42-66-97-83$

Science Counselor

Jerome J. Bosken 
PNL-9450-1

FRANCE

$\begin{array}{lll}\text { Population } & 1992 & 56 \text { million }\end{array}$

ENERGY

Electric Power Capacity $1992 \quad 105.7$ GWe

$55 \%$ nuclear

1995107.5 GWe

$2000 \quad 118.3$ GWe

$54 \%$ nuclear

Electric Power Production 1992

458.1 TWh

$74 \%$ nuclear

$15 \%$ hydro/geoth.

$8 \%$ coal

$2 \%$ oil

$1 \%$ gas

$1995 \quad 74 \%$ nuclear

$2000 \quad 74 \%$ nuclear

\section{NUCLEAR POWER}

Policy: Vigorous nuclear power program, scaled down recently to construction of less than one new reactor per year; commercialization of the breeder reactor; export of nuclear plants and services.

$\begin{array}{lrrrr}\text { Nuclear Power Capacity } & 1993 & 59.1 & \text { GWe } \\ & 1995 & 59.7 & \text { GWe } \\ & 2000 & 64.1 & \text { GWe } \\ \text { Reactor Mix } & & & \\ & 1993 & \text { GCR } & 1(1972) \\ & & \text { PWR } & 54(1972-93) \\ & & & 4(1995 / 99) \\ & \text { LMFBR } & 1 & (1974)^{(a)} \\ & & & 1(1994)\end{array}$

(a) Initial criticality for SuperPhenix in 1986. 


\section{INDUSTRIAL FUEL CYCLE}

Policy: Maintain full domestic fuel cycle capability and aggressive export of fuel cycle plants, equipment, and services (including uranium enrichment and spent fuel reprocessing).

Waste Management Strategy: HLW--vitrify and store in engineered storage facility for indefinite period, then emplace in geologic repository (granite or clay). LLW--immobilize in bitumen, concrete, or resin and dispose in engineered surface facility.

Cumnlative (PWR)

SF Arisings, $\mathrm{tU}$

$$
\frac{1990}{7,300} \quad \frac{1993}{10,000} \quad \frac{2000}{19,000}
$$

\section{Industrial-Scale Activities}

- Uranium mining and milling (tU/a): 1,710 (1993)

- Uranium enrichment (kSWU/a)

- Eurodif, gaseous diffusion: 10,800

- Fuel fabrication (tHM/a)

- $\mathrm{UO}_{2}: 1,600$

- MOX: 50 (LWR fuels)

- Spent fuel reprocessing (t/a)

- Marcoule: 400 (U metal fuels)

- La Hague: 1,200 (LWR fuels) 


\section{Major Milestones}

- UP2-800 reprocessing plant (La Hague)

- Melox (MOX fuel fabrication plant-Marcoule)

- TRU disposal facility

- Underground Research Laboratory (completion date uncerain)

- HLW (glass) disposal facility

\section{INTERNATIONAL RELATIONSHIPS}

\section{DOE/CEA Umbrella Agreement for Cooperative}

Radioactive Waste Management Technology Exchange

Term: $\quad 07-26-83$ to $07-26-93$ (extension in process)

Scope: Preparation/packaging, D\&D, waste/spent fuel storage, geologic disposal, transportation requirements; technical workshops in the areas of LLW and TRU waste management; exchange of waste repository site characterization technology and data for granite and salt host rocks.

Member of EC, IAEA and OECD/NEA; major role in Eurodif uranium enrichment consortium (COGEMA); partnership with German and British companies in United Reprocessors GmbH (COGEMA) and Nuclear Transport, Ltd. (Transnucléaire). 


\section{ORGANIZATION}

- CEA (Atomic Energy Commission)--controls practically all nuclear R\&D.

Nuclear Research Centers: Cadarache, Fontenay-aux-Roses, Grenoble, Valrho, Saclay.

- CEA INDUSTRY: Industrial group concerned with all industrial fuel cycle activities in France.

- COGEMA (CEA 89.2\%): nuclear fuel cycle.

- COMURHEX (COGEMA 100\%): uranium conversion.

- EURODIF (COGEMA 51.5\%): commercial enrichment.

- SICN (100\%), FRAGEMA (50\%), FBFC (49\%), COMMOX $(60 \%)$ - COGEMA subsidiaries: fuel fabrication.

- SGN, USSI (COGEMA part subsidiary).

- TECHNICATOME (CEA 90\%): design, construction, operation of fuel cycle and/or waste facilities.

- STMI (CEA 60\%): waste management, decontamination, dismantling services.

- TRANSNUCLÉAIRE (51\% COGEMA): transport.

- ANDRA (National Waste Management Agency)--controls long-term waste management, disposal included.

- EdF (Electricité de France, $100 \%$ government)--public power generation; owns and operates all nuclear plants except Phenix (50\% EdF, 50\% CEA) and SuperPhenix (NERSA: $51 \%$ EdF, $33 \%$ ENEL, 16\% RWE). 
Minister of Industry, Telecommunication and Tourism

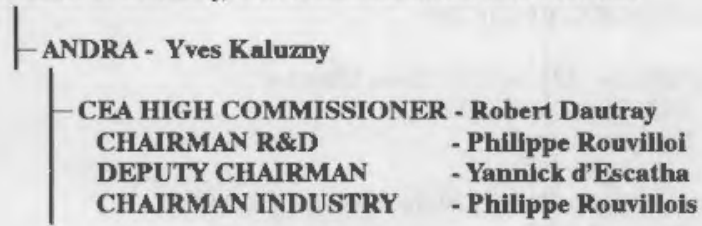

\section{CEA OPERATIONS UNITS}

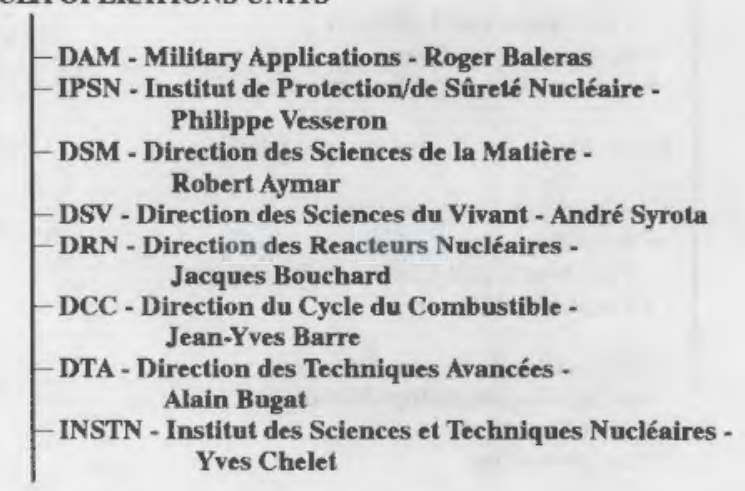

RESEARCH CENTERS

- CEN (see Page FR-6)

COGEMA

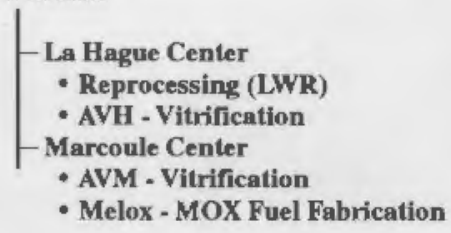




\section{CEA}

RESEARCH CENTERS (CEN)

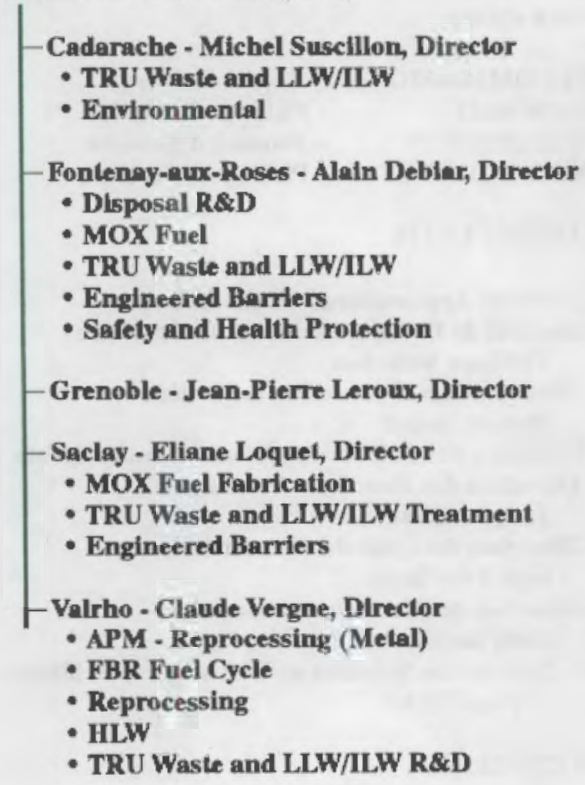




\section{ANDRA (National Agency for Radioactive Waste Management)}

Agence Nationale pour la Gestion des Déchets Radioactifs

Route Du Panorama Robert Schumann B.P. 38

92266 Fontenay-aux-Roses Cedex

France

Director

Deputy Director

Deputy Director
Tel: $\quad 33-1-46-54-7080$

Fax: $\quad 33-1-46-54-9925$

Henri-Edme Wallard

Armand Faussat

Yves Marque

Function: Design, site, construct, and manage long-term waste disposal facilities; establish radioactive waste packaging/disposal specifications and ensure compliance; contribute to $R \& D$ programs related to long-term waste disposal.

\section{Facilities}

- Centre de la Manche

B.P. 808

50448 Beaumont-Hague

Tel:

$33-16-33-52-78-65$

Mission: Disposal of ILW and LLW; capacity: $480,000 \mathrm{~m}^{3}$ (1988: $350,000 \mathrm{~m}^{3}$ in place; full and shut down in 1992 , to be closed permanently in 1994).

- Centre de l'Aube

\section{B.P. 7}

10200 Soulaines-Dhuys

Tel:

$33-16-25-92-33-00$

Mission: Replace La Manche site as disposal facility for ILW/LLW; located 120 miles east of Paris, in the province Aube; covers about 250 acres and will accommodate $1,000,000 \mathrm{~m}^{3}$ of ILW/LLW over a period of 30 years. 


\section{BRGM (Bureau of Geological and Mineral Research)}

Bureau de Recherches Géologiques

et Minières

B.P. 6009

45060 Orléans Cedex 2

France

Tel: $\quad 33-38-64-36-34$

Fax: $\quad 33-38-64-36-43$

Director

Managing Director, Geology

Jean Pierre Hugon

Waste Storage

H. Astie

Hydrogeology

P. F. R. Peaudecerf

Geotechnology

J. J. Collin

$\mathrm{Ph}$. Masure

\section{CEA (Atomic Energy Commission)}

Commissariat à l'Energie

Atomique (CEA)

Centre d'Etudes Nucléaires (CEN)

31-33, Rue de la Federation

75752 Paris Cedex 15

Tel:

33-1-40-56-10-00

France

Fax: $\quad 33-1-42-53-91-22$

Chairman

High Commissioner

Philippe Rouvillois Robert Dautray

Function: Responsible for R\&D related to all areas of the nuclear fuel cycle through activities of several operational units (scientific directorates), research centers, and wholly/partially owned industrial concerns. 


\section{CEA-IPSN (Institute for Nuclear Safety)}

Institute de Protection et de

Sureté Nucléaire (IPSN)

B.P. 6

92260 Fontenay-aux-Roses

France

Tel: $\quad 33-1-46-54-70-80$

Fax: $\quad 33-1-47-35-14-23$

Director

Dir., Nuc. Security Research

Dir., Safety

Safety Analysis

Dir., Safeguards

Health/Dosimetry

Environment/Installation

Research

Nuclear Materials

Philippe Vesseron

Michel Livolant

Daniel Queniart

Christian Devillers

Annie Sugier

R. Coulon (A)

Alain L'homme

A. Chalot

G. Déan

Function: Research and development in environmental safeguards/security.

\section{CEA/CEN-CA (Cadarache Nuclear Research Center)}

Centre d'Etudes Nucléaires de Cadarache

B.P. 1

13108 Saint Paul Lez Durance

France

Tel: $\quad 33-42-25-24-68$

Fax: $\quad 33-42-25-45-45$

Director

Michel Suscillon

Location: $65 \mathrm{~km}$ from Marseille-Marignane Airport (by car).

Waste Management R\&D: Treatment of TRU waste, LLW, and ILW; properties of non-HLW waste forms and waste isolation (radionuclide migration). 


\section{CEA/CEN-CA (contd)}

\section{Facilities}

- Solid Waste Treatment Pilot Plant (Prollxe, Elise)

Mission: TRU solid waste reduction by cryogenic crushing; $\mathrm{Pu}$ recovery by acid leaching.

Design Capacity: Eight 100-liter drums/batch, one batch every 2448 hours.

History: Startup, 1985.

- Bituminization Plant

Design Basis: Immobilize reactor wastes; twin-screw extruder; capacity, $260 \mathrm{~m}^{3} / \mathrm{a}$.

History: Startup, 1977.

- LLW Incinerator

- Resin Embedding Pilot Facility

- Solvent Incinerator

CEACEN-FaR (Fontenay-Aux-Roses Nuclear Research Center)

Centre d'Etudes Nucléaires

de Fontenay-aux-Roses

B.P. 6

92265 Fontenay-aux-Roses

France

Tel: $\quad 33-1-46-54-80-00$

Fax: 33-1-46-54-75-22

Director

Alain Debiar 


\section{CEA/CEN-G (Grenoble Nuclear Research Center)}

Centre d'Etudes Nucléaires

de Grenoble

B.P. 85

38041 Grenoble Cedex

France

Tel: $\quad 33-76-97-41-11$

Fax: $\quad 33-76-88-34-32$

Director

Jean-Pierre Leroux

Facilities

- Waste Resin Embedding Facility

\section{CEA/CEN-S (Saclay Nuclear Research Center)}

Centre d'Etudes Nucléaires

de Saclay

91191 Gif-sur-Yvette

Tel: $\quad$ 33-1-69-08-60-00

France

Fax: $\quad 33-1-69-08-79-90$

Director

Dir., Fuel Cycle (DCC)

WM/Reproc. Program Coord.

Eliane Loquet

Jean-Yves Barré

Guy Baudin

Facilities

- Bituminization Plant (radioactive).

- Melal Waste Melier (startup, 1985).

\section{CEA/CEN-VRH (Valrho Nuclear Research Center)}

Centre d'Etudes Nucléaires

de la Vallée du Rhône

B.P. 171

30205 Bagnols-sur-Ceze Cedex Tel: 33-66-79-60-00

Marcoule, France

Fax: $\quad 33-66-79-66-17$

(contd next page) 


\section{CEA/CEN-VRH (contd)}

Director

Mgr., Reprocessing/HLW

Deputy Mgr.

Manager, HLW

Decommissioning (DERD/NOIN)
Claude Vergne

Michele Viala

Claude Sombret

33-66-79-63-62

Jean-Pierre Moncouyoux

33-66-79-63-78

Claude Lafaille

\section{Facilities}

- APM (Cogema-operated demonstration reprocessing plant for FBR, MOX and high-burnup fuels)

Mission: Develop technology for FBR, MOX and high-burnup fuels.

Design Basis: PUREX flowsheet, mixer-settlers and pulsed columns; 5 tHM/a.

- PIVER (Hot Pilot Plant - Vitrification)

Mission: Test batch vitrification processes (1969-1973); produce samples for characterization and advanced (high-temperature) waste forms.

Design Basis: Pot calciner/melter; capacity, $90 \mathrm{~kg}$ glass/batch or 25-30 $\mathrm{m}^{3} \mathrm{HLW} / \mathrm{a}$; product, borosilicate glass blocks, $25 \mathrm{~cm}$ dia by $2.5 \mathrm{~m}$ high.

History: Decommissioned, then dismantled in 1991.

- PIVER II - Vitrification of HLW from APM. (Delayed)

- Hull Fusion Non-Radioactive Prototype - Startup, 1984.

- Hull Fusion Radioactive Facility in APM - Startup, 1993.

- PEV Prolotype (full-scale, non-radioactive R7/77 vitrification process) - Startup, 1984. 


\section{COGEMA (Fuel Cycle Company)}

Cogema Direction Generale

2, Rue Paul-Dautier

B.P. 4

78141 Velizy-Villacoublay Cedex

Tel: $\quad 33-1-39-46-96-41$

France

Fax: $\quad 33-1-34-65-14-52$

President, $\mathrm{CEO}, \mathrm{COB}$

Vice President

Ind. Director, Reprocessing

Jean Syrota

Christian Gobert

Jean-Louis Ricaud

Cogema, Inc.

7401 Wisconsin Ave.

Bethesda, Maryland 20814-3416

Tel: $\quad 301-986-8585$

Fax: $\quad 301-652-5690$

President, CEO

V.P.-Market Development

Michael McMurphy

Frank A. Shallo

NUMATEC, Inc.

Subsidiary of Cogema, Inc.

7401 Wisconsin Ave.

Bethesda, Maryland 20814-3416

Tel: $\quad 301-986-8585$

Fax: $\quad 301-652-5690$

President

William Gallagher

\section{COGEMA-LA HAGUE}

Cogema, Centre de La Hague

B.P. 508

50105 Cherbourg Cedex

France

Tel: $\quad 33-33-03-60-00$

Fax $\quad 33-33-44-71-77$

Director

Joël Pijsseiman

$33-33-03-60-01$

(contd next page) 


\section{COGEMA-LA HAGUE (contd)}

Fuel Cycle Program: Spent fuel reprocessing and HLW vitrification. The La Hague plant was originally designed to handle magnesiumclad U metal fuels from gas/graphite power reactors. Transfer of all reprocessing of gas/graphite fuels to Marcoule UP1 has been completed, and La Hague is devoted to treating LWR fuels with occasional FBR fuel campaigns through UP2.

\section{Facilities}

- UP2 (Fuel Reprocessing Plant)

Mission: Reprocess oxide fuels from LWRs and Phenix FBR (Phenix fuel has been reprocessed from 1979 to 1984, diluted with natural uranium fuel for criticality control).

Design Basls: PUREX flowsheet; oxide fuels: shear-leach HAO head-end; remote maintenance.

Capacity: $400 \mathrm{t} / \mathrm{a}$ of LWR fuels.

History: UP2 startup, 1967; HAO startup, 1976. From startup (06/76) through 12/93 total HAO throughput was 4,091 tHM fuel from LWRs and 10 tHM from Phenix.

- UP2-800 (Fuel Reprocessing Plant)

Mission: Reprocess $\mathrm{UO}_{2}$ and MOX fuels from French LWRs. Design Basis: Progressive expansion of UP2 plant from 400 to $800 \mathrm{tHM} / \mathrm{a}$ of LWR fuel started in 1984, to be completed in 1994. Chop leach head-end, PUREX flowsheet, AVM vitrification process [R7 vitrification plant: rotary calciner, metallic melter, capacity, $600 \mathrm{~m}^{3} / \mathrm{a}$ HLW feed three lines - 60 liters $/ \mathrm{h} \mathrm{HLW}, 25$ $\mathrm{kg} / \mathrm{h}$ glass; canister dimensions: $42 \mathrm{~cm}$ dia $\times 1.3 \mathrm{~m}$ high $(400 \mathrm{~kg}$ glass)].

Capacity: 800 tHM/a.

History: Stariup, 1994; R7 startup, 1989.

(contd next page) 


\section{COGEMA-LA HAGUE (contd)}

- UP3 (Fuel Reprocessing Plant) Mission: Reprocess LWR fuels.

Design Basis: Chop-leach head-end; PUREX flow-sheet; AVM vitrification process ( $T 7$ plant: identical to $R 7$ vitrification plant). Capacity: 800 tHM/a.

History: Startup, 09/90. Throughput of 1,626 tHM as of $12 / 93$.

- STE3 (Liquid Waste Treatment Facility)

Mlssion: Processing/encapsulation in bitumen of liquid low-and intermediate-level wastes from reprocessing of spent fuel at the La Hague installations.

History: Startup, 1989.

\section{COGEMA-MARCOULE}

Cogema, Centre de Marcoule

B.P. 170

30200 Bagnols-sur-Ceze

Tel: $\quad 33-66-79-60-00$

Marcoule, France

Fax: $\quad 33-66-89-38-50$

Location: Apprax. $70 \mathrm{~km}$ from Marseille-Marignane Airport (by train or car), near Avignon.

Director

Reprocessing Plant

AVM Manager
- Hughes Delaunay

Maurice Mellano

Jean-Claude

Batailles-Lannes

\section{Facilities}

- UP1 (Reprocessing Plant)

Mission: Reprocess magnesium-clad natural uranium metal fuels from military reactors.

Design Basis: Mechanical declad; PUREX flowsheet; contact maintenance.

Capacity: $400 \mathrm{tU} / \mathrm{a}$ of reactor fuel (gas/graphite).

Hislory: Startup, 1958; total gas/graphite power reactor fuels processed up to 12/93: $5,085 \mathrm{tU}$.

(contd next page) 


\section{COGEMA-MARCOULE (contd)}

- AVM (Ateliers de Vitrification de Marcoule) Mission: Demonstrate AVM process: vitrify Marcouje UP1 wastes.

Design Basls: Rolary calcinet feeding an induction- heated metallic melter; nominal capacity 30-liters/h HLW feed and $360 \mathrm{~kg} / \mathrm{d}$ (1 canister) borosilicate glass product; waste form, glass blocks $0.5 \mathrm{~m}$ dia $x 1.0 \mathrm{~m}$ high.

Hislory: Hot startup, 0678 ; as of $12 / 93,2,145$ glass canisters.

- Incineralar

- Bituminization Facibity

- Melox: MOX fuel fabrication (120 tIIM/a) - 1995.

\section{DAM (Direclarale of Military Applications)}

Direction des Applications Militaires

Commissariat à l'Energie Atomique

31 -33 Rue de la Fédération

B.P. 510

75752 Paris, Cedex 15 Tel: $33-1-40-56-10-00$

France

Fax: $\quad 33-1-40-56-14-29$

Director, Quality/Security Jean Ohmann

\section{ENSM (PARIS SCHOOL OF MINES)}

Ecole Nationale Superieure des Mines de Paris

Centre d'Informatique Géologique

35 Rue Saint-Honore

Tel: $\quad 33-1-64-22-48-21$

77305 Fontainebleau France

Fax: 33-1-64-22-39-02

Direclor, Math. Geol. Center

Depury Director

Dr. Ghislain de Marsily

Dr. G. E. Ledowx

(contd next page) 
ENSM (contd)

Waste Management R\&D: Geologic waste isolation (fluid flow, heat transport/mass transport studies--theoretical, lab/field tests).

\section{FBFC (Franco-Belge Company for Fuel Fabrication)}

Société Franco-Belge de Fabrication de Combustibles

Tour Fiat, Cedex 16

92084 Paris la Défense

France

Tel: $\quad 33-1-47-96-56-00$

Fax: $\quad 33-1-47-96-56-03$

Director General

Philippe Darmayan

Facilities

- Fuel Fabrication Plant (Romans, France)

Mission: Fabricate $\mathrm{UO}_{2}$ fuels for power reactors.

Design Capacity: 750 tHM/a.

- Fuel Fabrication Plant (Pierrelatte, France)

Mission: Fabricate $\mathrm{UO}_{2}$ fuels.

Design Capacity: $400 \mathrm{tHM} / \mathrm{a}$.

- Fuel Fabrication Plant (Dessel Belgium)

Mission: Fabricate $\mathrm{UO}_{2}$ fuels.

Design Capacity: 450 tHM/a. 


\section{GERMANY}

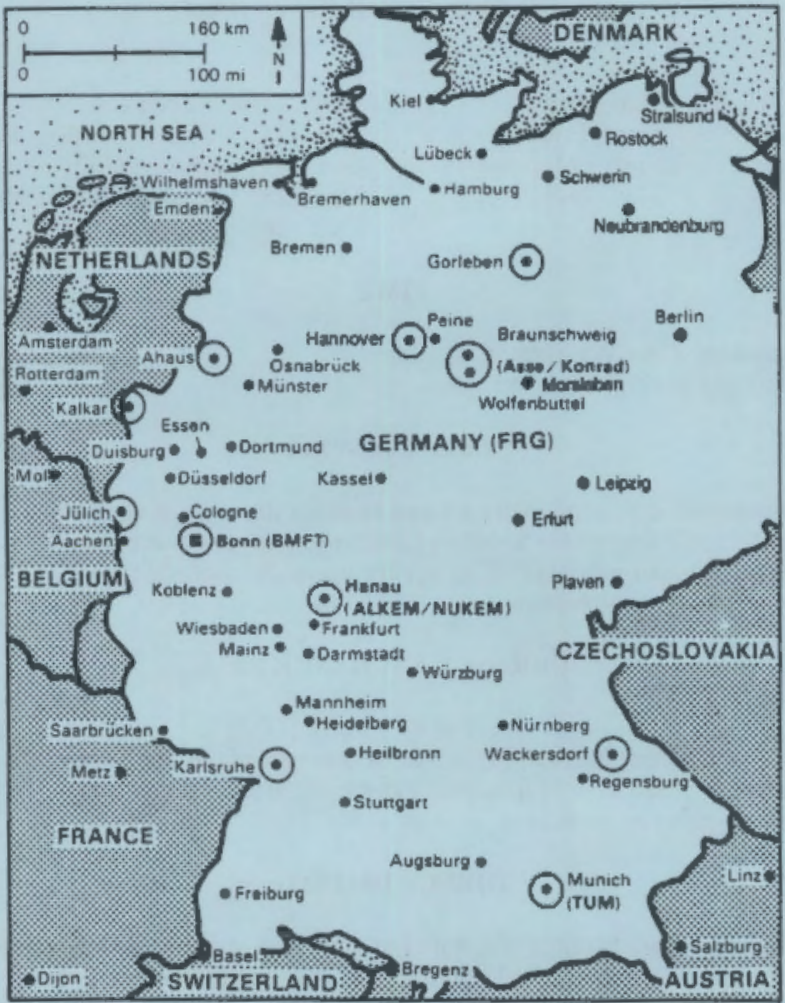


Population

1993

79 million

ENERGY

Electric Power Capacity

$\begin{array}{rrl}1993 & 118.5 & \text { GWe } \\ & 19 \% & \text { nuclear } \\ 1995 & 121.0 & \text { GWe } \\ & 18 \% & \text { nuclear } \\ 2000 & 126.2 & \text { GWe } \\ & 18 \% & \text { nuclear }\end{array}$

Electric Power Production 1993

$\begin{aligned} 533.3 & \text { TWh } \\ 57 \% & \text { coal } \\ 30 \% & \text { nuclear } \\ 6 \% & \text { gas } \\ 3 \% & \text { oil } \\ 3 \% & \text { hydro } \\ 1 \% & \text { solids } \\ 27 \% & \text { nuclear } \\ 25 \% & \text { nuclear }\end{aligned}$

NUCLEAR POWER

$\begin{array}{lrrl}\text { Nuclear Power Capacity } & 1995 & 22.5 & \text { GWe } \\ & 1995 & 22.5 & \text { GWe } \\ & 2000 & 22.5 & \text { GWe } \\ \text { Reactor Mix } & & & \\ & 1993 & \text { PWR } & 14 \text { (1972-89) } \\ & & \text { BWR } & 7(1975-85)\end{array}$

\section{INDUSTRIAL FUEL CYCLE}

Policy: Full commercial capability-enrichment, fuel fabrication, plutonium recycle to LWRs; reprocessing is to be handled by foreign plants.

Waste Management Strategy: Vitrification of HLW (by foreign plants) and interim storage of HLW glass; disposal of reprocessing wastes in future salt-dome repository; interim storage of ILW/LLW wastes; future disposal of reactor and decommissioning wastes in abandoned iron mine or salt repository. 
Population

1993

79 million

ENERGY

Electric Power Capacity

$\begin{array}{rrl}1993 & 118.5 & \text { GWe } \\ & 19 \% & \text { nuclear } \\ 1995 & 121.0 & \text { GWe } \\ & 18 \% & \text { nuclear } \\ 2000 & 126.2 & \text { GWe } \\ & 18 \% & \text { nuclear }\end{array}$

Electric Power Production 1993

$\begin{array}{rrl}1993 & 533.3 & \text { TWh } \\ & 57 \% & \text { coal } \\ & 30 \% & \text { nuclear } \\ & 6 \% & \text { gas } \\ & 3 \% & \text { oil } \\ & 3 \% & \text { hydro } \\ & 1 \% & \text { solids } \\ & 27 \% & \text { nuclear } \\ 1995 & 25 \% & \text { nuclear }\end{array}$

\section{NUCLEAR POWER}

Nuclear Power Capacity

$\begin{array}{rrr}1995 & 22.5 & \text { GWe } \\ 1995 & 22.5 & \text { GWe } \\ 2000 & 22.5 & \text { GWe } \\ & & \\ 1993 & \text { PWR } & 14(1972-89) \\ & \text { BWR } & 7(1975-85)\end{array}$

\section{INDUSTRIAL FUEL CYCLE}

Pollcy: Full commercial capability-enrichment, fnel fabrication, plutonium recycle to LWRs; reprocessing is to be handled by foreign plants.

Waste Management Strategy: Vitrification of HLW (by foreign plants) and interim storage of HLW glass; disposal of reprocessing wastes in future salt-dome repository; interim storage of ILW/LLW wastes; fnture disposal of reactor and decommissioning wastes in abandoned iron mine or salt repository. 
$\begin{array}{lll}\text { Cumulative SF Arisings (LWR) } & 1990 & 3,800 \mathrm{tU} \\ & 2000 & 8,950 \mathrm{tU}\end{array}$

Cumulative LLW/ILW Arisings $\quad 2000 \quad 160,000 \mathrm{~m}^{3}$ conditioned, radioactive waste with negligible heat production

Cumulative Heat-Producing 2000 $2,800 \mathrm{~m}^{3}$ conditioned, radioactive waste with heat production

\section{Industrial-Scale Activities}

- Fuel fabrication capacity

- $\mathrm{UO}_{2}$ fuel: $1500 \mathrm{tU} / \mathrm{a}$

- MOX fuel: 130 tHM/a-LWR/10 tHM/a-FBR fuel elem. (no operating license)

- AFR spent fuel storage capacity

- 1,500 t, dry storage (Gorleben)

- 1,500 t, dry storage (Ahaus)

\section{Major Milestones}

- Acceptance of HLW from Cogema/La Hagne and BNFL/Sellafield

- Konrad (iron mine) repository (date pending)

- Gorleben repository, HLW 


\section{INTERNATIONAL RELATIONSHIPS}

\section{DOE/BMFT Agreement for Cooperative Radioactive Waste Management Technology Exchange}

Term: 12-20-74 to 06-30-91 (In process of being extended)

Scope: Geologic disposal in salt deposits; retrievable surface storage; D\&D; operational aspects of LL/ILW storage and disposal; transportation. Emphasis: Waste treatment technology (design/operation of HLW vitrification pilot plants, conditioning of LLW/TRU wastes, waste form characterization), waste package development; collaboration with in-situ tests at Asse salt mine; U.S. observation of shaft drilling at the Gorleben repository site; cooperation in tests of transport/storage casks, and waste transportation studies.

Member of EC, IAEA, and OECDNEA. Cooperative agreements and joint projecls as well as commercial activities with numerous countries.

\section{ORGANIZATION}

- Federal Government

- Coordinate nuclear program

- Sponsor R\&D

- Construct/operate radioactive waste disposal facilities

- Establish licensing requirements

- Stales (Länder)

- License nuclear installations

- Provide LLW interim slorage

- Utilities

- Provide spent fuel/reactor waste storage, contract for reprocessing and waste treatment

- Pay for waste transport and disposal 
GOVERNMENT RESPONSIBILITIES--

NUCLEAR FUEL CYCLE/WASTE MGMT.

BMFT (Federal Ministry for Research and Technology)

- Government Fuel Cycle/Waste Management R\&D Program Administration

- GSF/IrT

- Geologic Waste Disposal R\&D

- Supporting Lab Work - Salt Properties

- Asse Studies

KRK

- LWR Fuel Cycle Waste Treatment/Packaging R\&D

- LWR SF Management Alternatives - R\&D

- HLW Vitrification R\&D

- Support Work - Geological/Direct Disposal of Waste

- KFA

- Waste Treatment

- Support Work - LLLJLW Disposal, Including HTGR Fuel Elements

BMW (Federal Ministry for Economics)

BGR

- Geologle Survey

- Salt Dome Reposilory RAD (Salt Properties, Rock Mechanics) 


\section{GOVERNMENT RESPONSIBILITIES--}

NUCLEAR FUEL CYCLE/WASTE MGMT. (contd)

\section{BMU (Fed. Ministry-Environmental Protection/Reactor Safety)}

- Storage/Transp./Disposal of Rad. Wastes

- Supervision of State Licensing Procedures

- Nuclear Safety/Radiation Protection

RSK (Reactor Safety Commission)

SSK (Radiation Protection Commission)

$\mathbf{B} \mathbf{S}$

- Transportation/Storage/Licensing

- Responsibility for Repository Construction/Operation

\section{DBE}

- Construction/Operation (Reposilories)

- Gorleben and Konrad Projects

- Morsleben LLW Disposal Facility (ERAM)

LÄNDER (Stale Governments)

- Licensing of Nuclear Installations

NMU (Lower Saxony Ministry of Environment)

- Licensing of Finel Reposilories (Konrad and Gorleben)

- Morsleben LLW Disposal Facility (ERAM) 


\section{INDUSTRIAL/UNIVERSITY RESPONSIBILITIES}

DWK - Owned by Nuclear Utilities

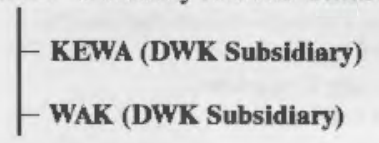

NUKEM - Owned by RWE

- LLW/TRU waste treatment R\&D facility design

- R\&D--SF packaging for disposal

GNS - Owned by Nuclear Utilities

- Waste treatment/conditioning

- Transportation of radioactive materials

- Shipping cask development

- Engineering/D\&D services

BLG (GNS Subsidiary)

- Operation of Gorleben SF/LLW Storage Facilities

- Construction of PKA

- BZA (GNS Subsidiary)

- Operation of Ahaus SF Interim Storage Facility

NCS - Nuclear Cargo Service

- Transportation of radianclive matertals (owned by Deulsche Bahn AG)

SBH - Owned by Siemens AG

- Fabrication of uranium/MOX fuels, Including R\&D/waste management

TUM - Technical University Munich

- Actinide Chemistry R\&D 


\section{BAM (Federal Materials Research/Testing Institute)}

Bundesanstalt für Materialforschung und -prüfung (BAM)

Unter den Eichen 87

12205 Berlin, Germany

Tel: $\quad 49-30-8104-0$

Fax: $\quad 49-30-811-5066$

Function: Testing and evaluation of materials used in nuclear programs.

\section{BS (Federal Institute for Radiation Protection)}

Bundesamt für Strahlenschutz

Postfach 100149

38201 Salzgitter, Germany

Chief Executive

Vice-President

BIS, Department Nuclear

Waste Disposal/Transport

Bundesallee 100

38116 Braunschweig, Germany

Director

Dir., Project Mgmt.

Dir., Waste Disposal/Safety

Radioactive Waste

Geoscience

Radiology/Radiation Protection

System Analysis

Dir., Transport/Storage of

Rad. Mtls./Fuel Cycle
Tel: $\quad 49-5341-188-0$

Fax: $\quad 49-5341-188-188$

Prof. Dr. Alexander Kaul

H. Rósel

Tel: $\quad 49-531-592-7601$

Fax: $\quad 49-531-592-7614$

Prof. Dr. Helmut

Röthemeyer

Dr. Bruno Thomauske

Dr. Heinrich Illi

Dr. Peter W. Brennecke

49-531-592-7641

Dr. Gerhard Stier-Friedland

Dr. Dietrich Ehrlich

TBD

Prof. Dr. Wilhelm Collin

(contd next page) 


\section{$\underline{\text { BIS }}$ (contd)}

Function: Execution of the federal responsibilities concerning testing/standards for radiation protection, nuclear safety, radioactive waste disposal, and transport/storage of radioactive materials; in particular, the responsibility for construction and operation of repositories.

\section{Facilities}

- Gorleben Sile (planned repository), $100 \mathrm{~km}$ northeast of Braunschweig.

Mission: Disposal of all types of solid radioactive waste.

Reposilory Concept: 300 to $600 \mathrm{~m}$ deep boreholes in tunnel floors at depths of about $850 \mathrm{~m}$ in the Gorleben salt dome.

Milestone: Startup of disposal, 2010.

- Konrad Site (planned repository in a former iron ore mine), 10 $\mathrm{km}$ southwest of Braunschweig.

Mission: Disposal of waste with negligible thermal impacts on host rock formation.

Milestone: Startup of disposal, 1997/98.

\section{BGR (Federal Institute for Geosciences and Natural Resources)}

Bundesanstalt für Geowissenschaften und Rohstoffe

Stilleweg 2

Postfach 510153

30655 Hannover, Germany

Tel:

Fax:
49-511-643-0

49-511-643-2304

(contd next page) 
BGR (contd)

Director, Engineering,

Geology/Geotechniques

Rock Mechanics

Engineering Seismology

Salt Mechanics

Mining Rock Mechanics

Salt Geology

Numerical Modeling

Hydrogeology

Groundwater Geophysics

Prof. Dr. Michael Langer

Prof. Dr. A. Pahl

Dr. R. Lüdeling

Dr. H. Albrecht

Dr. D. Meister

Dr. W. Jaritz

Dr. Manfred Wallner

Dr. H. Vierhuff

Dr. W. Giesel

Functlon: Responsible to BMWI for all geological/geotechnical aspects related to planning, construction/operation of a final repository for radioactive wastes; conduct special research for BMU.

\section{BMFT (Federal Ministry for Research and Technology)}

Bundesministerium für Forschung

und Technologie

Heinemannstrasse 2

Postfach 200240

53175 Bonn, Germany

Tel: $\quad 49-228-591$

Fax: $\quad 49-228-59-3605$

Minister, Science/Technology

Director General, Energy

Fuel Cycle/D\&D

Dr. Paul Krüger

Dr. Eckhard Lübbert

Dr. Klaus Komorowski

49-228-59-3759

Waste Mgmt./D\&D/Fuel

Dr. Bernd Schillert

49-228-59-3757

U Ènrichment/Safeguards

Dr. A. H. Remagen 49-228-59-3755

Waste Disposal

Dr. Diethard Lummerzheim 49-228-59-3762

Direct Disposal

Dr. Hans G. Riotte

Geological Disposal

49-228-59-3761

W. Busch

49-228-59-3764

Function: Responsible for R\&D programs on fuel cycle and radioactive waste management. 
BMU (Federal Ministry for Environmental Prolection/Reactor Safety

Bundesministerium für Umwelt, Naturschutz und Reaktorsicherheit Kennedyallee 5

53175 Bonn, Germany

Tel: $\quad 49-228-305-0$

Fax: $\quad 49-228-305-3225$

Minister

Dir. Gen., Nuc. Installation

Safety/Radiation Protection/

Nuclear Fuel Cycle

Dir., Nuc. Installation Safety

Director, Radiation Protection

Prof. Dr. Klaus Töpfer

Walter Hohlefelder

Director, Fuel Cycle

Dr. Gast

Dr. Gallas

49-228-305-2805

49-228-305-2905

Dr. Arnolf Matting 49-228-305-2950

Policy

Dr. Bröcking 49-228-305-2930

International Relations

Christian Breest 49-228-305-2800

Fuel Supply Arno Ehret 49-228-305-2831

Reprocessing/Conditioning

Dr. K. H. Berg 49-228-305-2821

Treatment/Storage/Transp.

Dr. Herbert Dreisvogt 49-228-305-2721

Final Repository

Dr. Manfred Bloser 49-228-305-2951

Chairman, Reactor Safety Commission (RSK) Chairman, Radiation Protection Commission (SSK) Prof. Dr. Adolf Birkhofer Prof. Dr. Christian Steffen

Function: Responsible for storage, transportation, and disposal of radioactive wastes; supervision of state licensing procedures; federal standards for nuclear safety and radiation protection. 


\section{DBE (German Company for Construction/Operation} of Waste Disposal Facilities)

Deutsche Gesellschaft zum Bau und Betrieb von Endlagern für Abfallstoffe mbH

Woltorfer Strasse 74

31224 Peine, Germany

Managing Directors

Project Gorleben, Mgr.

Project Konrad, Mgr.

Project-Related R\&D, Mgr.
Tel: $\quad 49-5171-43-1$

Fax: $\quad 49-5171-43-218$

Dr. Jürgen P. Lempert

Manfred Florl

Dr. Hans-Jürgen Krug

Wolfgang Schulz

Rüdiger Putzer

49-5171-43-250

49-5171-43-310

Hans-Jürgen Engelmann 49-5171-50-3370

Activities: Conceptual design of repositories; site investigations; construction of surface/subsurface facilities for repositories; heat-related stress analyses; development of emplacement techniques; construction of emplacement equipment; risk assessments; safety analysis operational and post-operational phases; design/construction of engineered barriers.

\section{DWK (German Fuel Reprocessing Company)}

Deutsche Geselischaft für

Wiederaufarbeitung

von Kernbrennstoffen mbH

Baringstrasse 6

Tel: $\quad 49-511-3668-0$

30022 Hannover, Germany

Fax: $\quad 49-511-3668-207$

Manager

Bernd Zur Nedden

Function: Support KEWA and WAK; resolve issues from consequences of cancellation of reprocessing plant Wackersdorf and resulting transfer of site from "nuclear" to "industrial"; plan for decommissioning/dismantling of WAK pilot-scale reprocessing plant. 


\section{ERAM (LLW Repository)}

Endlager für Radioaktive

Abfälle Morsleben

Am Schacht 105

39343 Morsleben, Germany

Tel: $\quad 49-39050-8-0$

Fax: $\quad 49-39050-300$

Manager

Dr. Klaus Ebel

49-39050-8-200

Function: Final repository for LLW of the former East Germany, now operated by DBE under contract to BrS.

\section{GNS (Company for Nuclear Service)}

Gesellschaft für

Nuklear-Service mbH

Zweigertstrasse 28

45130 Essen, Germany

Tel: $\quad 49-201-7220-0$

Fax: $\quad 49-201-7220-181$

Managing Directors

Dr. Wolfgang Hawickhonst

Dr. Klaus Janberg

49-201-7220-102

Norbert Semann

Function: Service to nuclear facilities, including waste treatment/ conditioning, transportation of radioactive materials, shipping cask development, and facility dismantling.

Ownership: Nuclear utilities.

(contd next page) 


\section{GNS (contd)}

\section{Facilities}

- AFR Spent Fuel Storage Facilities (Gorleben and Ahaus sites, operated by GNS subsidiaries, BLG, and BZA, respectively) Design Basis: Dry storage in CASTOR casks - 400 casks (in $600 \mathrm{x}$ $125 \times 62$-ft-high building).

Capacity: 1500 t each facility.

History: Startup of AFR at Gorleben has been delayed due to litigation. Ahaus went into operation in 1992.

- PKA Pilot Fuel Conditioning Plant (Gorleben)

Mission: Demonstration of SF conditioning and encapsulation to meet the requirements for interim storage and final disposal. Design Basis: Hot cell with installations for rod consolidation, compaction of fuel assembly hardware, loading of canisters; maximum throughput 35 tHM/yr.

Milestone: Startup, 1996.

\section{GRS (Company for Reactor Safety)}

Gesellschaft für

Reaktorsicherheit mbH

Schwertnergasse 1

50667 Köln, Germany

Tel: $\quad 49-221-2068-0$

Fax: $\quad 49-221-2068-442$

General Manager

Prof. Dr. Adolf Birkhofer

Function: Provide technical support to BMU and other regulatory/ licensing entities concerned with reactor safety issues. 
GSF/IrT (Research Center for Environmental Sciences/ Institule for Underground Disposal

GSF - Forschungszentrum für Umwelt

und Gesundheit, GmbH

Institut für Tieflagerung

Theodor-Heuss-Strasse 4

P.O. Box 2163

38122 Braunschweig, Germany

Tel: $\quad 49-531-8012-1$

Fax: $\quad 49-531-8012-200$

Dir./IFT, Head/Disposal

Technology

Engineering Development

Geotechnology

Test Fields

Geophysics

Dir./IfT, Head/Disposal Safety

Prof. Dr. Klaus Kühn

49-531-8012-231

Alfred Beinlich

Manfred W. Schmidt

Tilmann Rothfuchs

Dr. Dieter Flach

Dr. Wernt Brewitz

49-531-8012-239

Safety Analysis

Chemical Waste

Dr. Richard Storck

Geochemistry

Geology/Hydrogeology

Data Processing

Dir./Project Management

ILW/HLW Projects

Direct Disposal Project

Asse Projects

Konrad/Gorleben Work

Test Dam Project

Long-Term Safety Projects

Administration/Infrastructure

Public Relations

Head/Mine Operations

Dr. Thomas Brasser

Dr. Hermann J. Gies

Dr. Konrad Klarr

Gert Ohme

Dr. Rolf Stippler

49-531-8012-220

Dr. Ingo Müller-Lyda

Jürgen Kunze

Vacant

Dr. Wolfgang Bode

Dr. Helmut Fleck

Dr. Peter Faber

Erwin Sölter

Rainer Gömmel

Klaus Dürr

Technical Planning 49-531-8012-211

Helmut Kolditz

(contd next page) 


\section{GSF/IfT (contd)}

Waste Management R\&D: Development/testing of techniques for safe, final geologic disposal of radioactive and chemical toxic wastes; acquisition of data for planning, construction, and operation of underground repositories. Safety analyses of long-term performance for the post-operational phase of underground repositories.

\section{Schachtanlage Asse}

38319 Remlingen, Germany

Mine Manager

Radiation Protection

Mine Survey
Tel: $\quad 49-5336-891$

Volker Schauermann

Herbert Meyer

Dr. Gerd Hensel

\section{Facilities}

- Asse Salt Mine (12 km southeast of Wolfenbüttel) Mission: In-situ testing and disposal technology development for a salt dome repository; through 1978, disposal of LLW and ILW. History: Startup, 1967.

- Mineralogical and Geochemical Laboratories (Braunschweig)

- Rock Mechanics Laboratory (Braunschweig)

\section{KEWA (Fuel Cycle Consulting Company)}

KEWA Kembrennstoff

Wiederaufarbeitungstechnik $\mathrm{GmbH}$

Baringstr. 6

Postfach 3245

30032 Hannover, Germany

Tel: $\quad 49-511-3668-0$

Fax: $\quad 49-511-3668-203$

Tech./Managing Director

Emst Robinson

49-511-3668-240

Function: Consulting/design services in reprocessing of LWR fuel elements, waste treatment safety and related areas such as remote handling, environmental protection, and others. KEWA is a DWK subsidiary. 


\section{KFA (Jülich Research Center)}

Forschungszentrum Jülich $\mathrm{GmbH}$

Postfach 1913

52245 Jülich, Germany

Tel: $\quad 49-2461-61-0$

Fax: $\quad 49-2461-61-5327$

Director, Institute of

TBD

Chemical Technology (ICT)

Director, Institute of

Prof. Dr. Hubertus Nickel

Reactor Materials (IRW)

ILW/SF/HTGR Fuel Disposal

49-2461-61-3058

Dr. Heiner Brücher

49-2461-61-6409

Waste Treatment (ZFK-DE)

Quality Assurance (PKS)

Dr. Stephan R. Halaszovich

49-2461-61-5288

Dr. Reinhard Odoj

49-2461-61-6190

Hot Cell Facility (GHZ)

Günter Pott

49-2461-61-3196

Function: Develop advanced waste management technologies.

Activities: Hot cell experiments dealing with the development of advanced ILW/HLW conditioning processes; characterization of waste products/packages; conditioning of radioactive wastes generated from research center; development/demonstration of quality assurance measures for waste packages; retrievable in-situ testing of ILW disposal techniques in Asse salt mine, including direct disposal of HTR fuel elements; LLW incineration using Jülich furnace design. 


\section{KחK (Karlsruhe Nuclear Research Cenler)}

Kernforschungszentrum

Karlsruhe GmbH

Postfach 3640

76021 Karlsruhe, Germany

Tel: $\quad 49-7247-821$

Fax: $\quad 49-7247-82-5070$

(Convenient route from U.S. is by plane to Frankfurt, then by train or car to Karisruhe.)

Director, Inst. for Hot Chem.

Prof. Klaus Ebert

Hot Chemistry (IHCh)

Director, Institute for Nuc.

Waste Technology (INE)

Process Engineering

49-7247-82-2400

Prof. Dr. Jä-ii Kim

49-7247-82-2230

Dr. S. Weisenburger

49-7247-82-4288

Director, Institute for

Radiochemistry (IRCh)

Director, Ctrl. Eng. Dept. (IT)

Prof. Ache

49-7247-82-3200

Dr. Hermann Rininsland 49-7247-82-3000

Remote Handling

G. Boehme

49-7247-82-2600

WAK Decommissioning

Walter Müller-Dietsche 49-7247-82-4050

Program Management

"Entsorgung" (PTE)

Dr. Klaus-Detlef Closs

49-7247-82-5790

(contd next page) 


\section{$\underline{\mathbf{K R K}}$ (contd)}

\section{Facilities}

- BEATE Facility

Mission: Aerosol source term destination and VOG behavior.

Design Basis: Stirring and transport of liquids by air and steam.

History: Startup, 1983 (program completion, 1990/91); in standby.

- Ceramle Melter

Mission: HLW vitrification process development with ceramic melter for the PAMELA pilot plant.

Design Basis: Liquid-fed, joule-heated melter;

PAMELA capacity: 30 -liter/h HLLW or $30 \mathrm{~kg} / \mathrm{h}$ glass.

History: Startup PAMELA melter, 1976; Mark 1, 1985, hot;

Mark 2, 1990, cold.

- Waste Concreting Plant (radioactive)

Mission: Immobilize KfK ILW.

Design Capacity: $2.5 \mathrm{t} / \mathrm{d}$ waste.

History: Startup, 1977.

\section{NMU (Lower Saxony Ministry of Environment)}

Niedersächsisches Umweltministerium

Archivstrasse 2

Postfach 4107

Tel: $\quad 49-511-104-0$

30041 Hannover, Germany

Fax: 49-511-104-3399

Minister

Dir., Nucl.Energy/Rad.Protection

Monika Griefahn

Final Repositories

Klaus-Dieter Becherer

Dr. Klaus-Arno Beckers

49-511-104-3550

NFC (WM/Reprocessing/SF)

Dr. Dietmar A. Kopp

49-511-104-3503

Function: State authority for licensing of nuclear facilities in Lower Saxony, including planned repositories at Gorleben and Konrad. 


\section{NUKEM}

NUKEM GmbH

Industriestrasse 13

P.O. Box 1313

63754 Alzenau, Germany

Managing Directors

Process Engineering

Fuel Cycle Services

Non-Destructive Testing

Environmental Technology

Solar Energy Technology

Nuclear Engineering
Tel: $\quad 49-6023-91-01$

Fax: $\quad 49-6023-91-1222$

L. Aumüller, H. A. Pirk

H. W. Binzel,

Dr. P. Schmidt

O. Pfahls

Dr. H. Keese

H. Hüschelrath

Dr. P.G. Maurer

Dr. W. Hoffmann

Dr. E. Wehner

Function: Nuclear fuel cycle services; environmental technology; hazardous waste/toxic residues treatment; off-gas/exhaust gas treatment; mist eliminator filters; general/nuclear process engineering; safety engineering; container systems.

\section{DETEC}

Decommissioning Technologies, GmbH Industriestrasse 13 Tel: $\quad 49-6023-91-04$ 63755 Alzenau, Germany Fax: $\quad 49-6023-91-1222$

Managing Directors

Gerwin Rasche Dr. Erwin Wehner

Function: Provide services for D\&D, engineering, remote handling systems. 


\section{SBH}

Siemens AG Brennelementewerk Hanau Postfach 110060 Tel: $\quad 49-6181-58-0$ 63434 Hanau, Germany Fax: $\quad 49-6181-58-3502$

Production Manager Jürgen Krellmann 49-6181-58-4599

Chemistry/Waste Management

Function: Fabrication of uranium fuel for BWR/PWR and MOX for BWR/PWR, including R\&D on waste management.

\section{Facilities}

- Fuel Fabrication Plants

Capacity $\mathrm{UO}_{2}-1500$ tHM/a LWR fuel.

MOX - $130 \mathrm{tHM} / \mathrm{a}$ LWR fuel or $10 \mathrm{tHM} / \mathrm{a} \mathrm{FBR}$ fuel (plant constructed, no operating license)

\section{TUM (Technical Universlty Munich)}

Technische Universität München

Institut für Radiochemie

Walther-Meissner-Strasse 3

85748 Garching b. München

Germany

Tel: $\quad 49-89-3209-220$

Fax: $\quad 49-89-3209-2204$

Director

Prof. Franz Baumgärtner 


\section{VKTA (Nuclear Engineering/Analytics Company)}

Verein für Kernverfahrenstechnik und Analytik Rossendorf e.V.

Postfach 511019

01314 Dresden, Germany

General Manager

Waste Treatment

Nuc. Analytics

Radioactive Preparation
Tel: $\quad 49-351-591-3350$

Fax: $\quad 49-351-460-5812$

Prof. Dr. Wolf Häfele

49-351-591-2350

Dr. Frank Schumann

49-351-591-2671

Dr. H. Steinkopf

49-351-591-2290

Dr. K. Jantsch

Activities: Decommissioning nuclear facilities of the former Central Institute for Nuclear Research (ZIK Rossendorf): zero power reactors, RFR research reactor $(10 \mathrm{MW})$, and special radioisotope production facilities; waste treatment/nuclear services; nuclear/chemical analyses; assessment of industrial/mining wastes; radioisotope production.

\section{Facilities}

- ABŨS (Waste treatment plant) Laboratory- and pilot-scale R\&D of LLW/HLW treatment processes: volume reduction (evaporation, press compaction, solidification); conditioning, using novel (remote) handling techniques ( 2 hot cells). Completed in 1991; hot operation in 1996.

- SWA (Waste water processing/treatment plant) Treatment consisting of mechanical filtration, ion exchange for low-salt wastes, and evaporation for high-salt wastes and wastes containing boric acid.

- Radiolsotope Production Facilities 


\section{WAK (Fuel Reprocessing Company)}

Wiederaufarbeitungsanlage Karlsruhe

Betriebsgesellschaft mbH

Postfach 1263

76339 Eggenstein-Leopoldshafen

Germany

Tel: $\quad$ 49-7247-2881

Fax: $\quad 49-7247-4755$

Location: WAK and the WAK plant are located on the site of the Karlsruhe Nnclear Research Center (WAK is a subsidiary of DWK).

Chief Executive

Technical Oversight

Plant Manager

Decommissioning

Waste Conditioning/Transport

\section{R. Heere}

Horst P. Wiese

49-7247-88-2201

49-7247-88-2118

Dr. Martin Weishaupt

49-7247-88-2298

Dr. Xlaus Eiben

49-7247-88-2117

Dr. Joachim Fleisch

$49-7247-88-2230$

\section{Facilities}

- WAK Reprocessing Plant (owned by KfK).

Mission: Reprocess $\mathrm{UO}_{2}$ and MOX fuels; recover plutoninm for recycle; test advanced technology.

Design Rasis: Chop-leach head-end; PUREX process; capacity, $0.175 \mathrm{tHM} / \mathrm{d}$.

History: On-line from $09 / 71$ to early 1980 , when it was shut down for dissolver replacement; operation resumed 10/82; total throughput to $12 / 90$, approx. 210 tHM (130 tHM from LWR fuel); shut down 12/31/90, decommissioning/dismantling plans in progress.

(contd next page) 


\section{WAK (contd)}

- PAMELA Pilot Plant (Mol, Belgium--ownership transferred to Belgoprocess in 1986; operated by WAK/Belgoprocess team) Mission: Demonstrate ceramic melter and VITROMET production with stored Eurochemic HLLW.

Design Basis: Liquid-fed ceramic melter, $0.72 \mathrm{~m}^{2}$ surface area; capacity, 36 liters $/ \mathrm{h}$ feed, $25 \mathrm{~kg} / \mathrm{h}$ glass ( 3 canisters $/ \mathrm{d}$ of $150-\mathrm{kg}$ glass/canister); product, borosilicate glass blocks, 0.3 -m dia by 1.2 m high.

History: Hot operation, startup 1985 (KfK development); as of August 1991: $910 \mathrm{~m}^{3}$ waste vitrified, 2,180 canisters filled; tanks now empty, plant in standby condition. 


\section{INDIA}

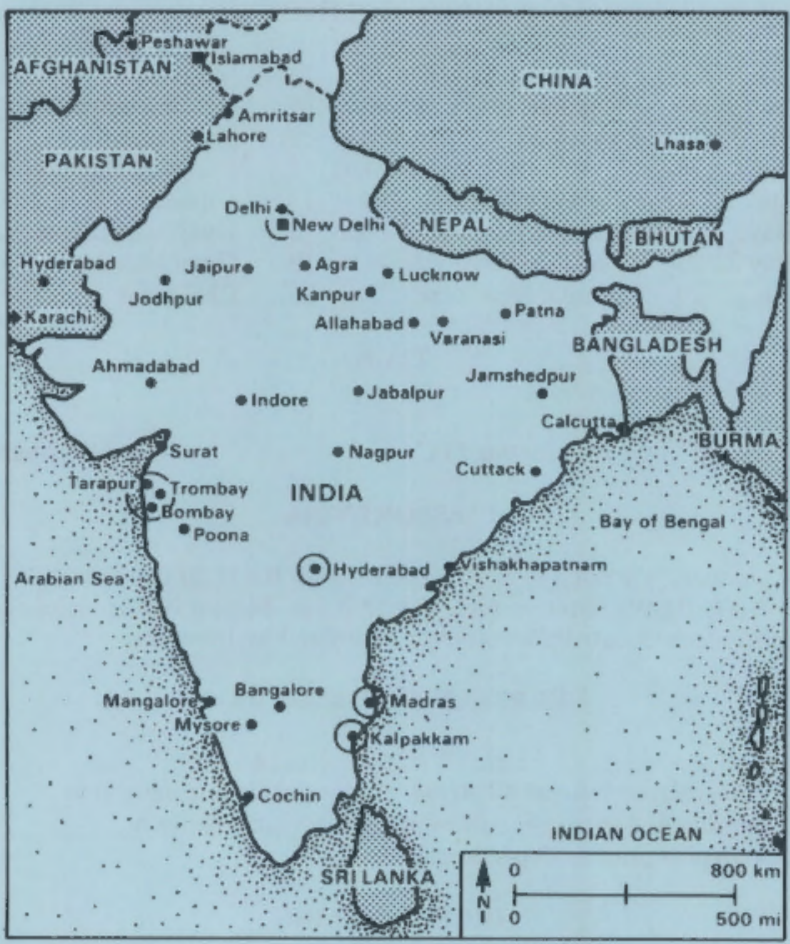




\section{INDIA}

\section{MAJOR PUBLIC HOLIDAYS (1994)}

$\begin{array}{lrllrl}\text { Jan } & 1 & \text { New Year } & \text { Jun } & 20 & \text { Muharram } \\ \text { Jan } & 26 & \text { Republic Day } & \text { Aug } & 15 & \text { Independence Day } \\ \text { Mar } 13-15 & \text { Ramadan } & \text { Aug } & 29 & \text { Jonashtami } \\ \text { Mar } & 26 & \text { Holi } & \text { Oct } & 2 & \text { Gandhi's Birthday } \\ \text { Apr } & 1 & \text { Good Friday } & \text { Oct } & 13 & \text { Dashera } \\ \text { May } & 5 & \text { Buddha Purima } & \text { Nov } & 2 & \text { Festival of Lights } \\ \text { May 22-23 } & \text { Sacrifice Feast } & \text { Nov } & 18 & \text { Guru Nanak's B'day } \\ \text { Jun } & 10 & \text { Islamic New Year } & \text { Dec } & 25 & \text { Christmas }\end{array}$

\section{TIME}

Standard Time Washington, D.C.

+10.5 hours

\section{PASSPORT/VISA}

A passport is needed to depart and re-enter the U.S.; in addition, a visa is currently required for a visit to India. Most travel agencies can provide up-to-date information concerning requirements.

\section{CURRENCY EXCHANGE RATE}

1 U.S. $\$=31.13$ Rupee

per Wall Street Journal, 01/31/94. As rates fluctuate daily, it is recommended to obtain current rates from local banks or newspapers prior to departure.

\section{DIRECT DIALING}

Individual numbers for direct dial to India are complete as listed, after dialing international access code: 011. Country code is 91; listed local numbers include city code.

\section{U.S. EMBASSY - NEW DELHI}

American Embassy

Shanti Path

Chanakyapuri 110021

Tel: $\quad 91-11-687-2028$

New Delhi, India

Fax: $\quad 91-11-687-6033$

Science Counseior

Dr. Paul C. Maxwell 
819 million

\section{ENERGY}

$\begin{array}{lcrl}\text { Electric Power Capacity } & 1992 & 69 & \text { GWe } \\ & & <3 \% & \text { nuclear } \\ & 1997 & 100 & \text { GWe } \\ <3 \% & \text { nuclear } \\ & & & \\ \text { Electric Power Production } & 1992 & 302.7 & \text { TWh } \\ & & 76 \% & \text { coal } \\ & & 22 \% & \text { hydro } \\ & 1997 & 10 \% & \text { nuclear } \\ & 1997 & & \end{array}$

\section{NUCLEAR POWER}

Policy: Heavy dependence on nuclear power to augment the nation's electric power generating capacity. A three-phase program-first phase, reactors fueled with natural uranium; second phase, FBRs fueled with Pu produced by first-phase reactors; third phase, self-sustaining thorium-uranium-cycle reactors.

Due to resource and technical problems, it is doubtful that 1997 nuclear power forecasts (end of 8th five-year plan) can be met, including the commissioning of the 500 MWe FBR by the year 2000 .

$\begin{array}{lccl}\text { Nuclear Power Capacity } & 1993 & 1.7 & \text { GWe } \\ & 1995 & 2.2 & \text { GWe } \\ & 2000 & 3.5 & \text { GWe } \\ \text { Reactor Mix } & 1993 & \text { BWR } & 2(1969) \\ & & \text { HWR } & 7(1973-93) \\ \text { Reactor Development } & 1985 & \text { FBR } & 7(1994-01) \\ & 2000 & \text { FBR } & 500 \text { MWe commercial }\end{array}$

\section{INDUSTRIAL FUEL CYCLE}

Policy: Achieve self-sufficiency in CANDU-type and LWR fnel cycle--uranium mining and milling, conversion to $\mathrm{UO}_{2}$, fuel 
fabrication, reprocessing (in small plants adjacent to power stations); if enriched $\mathrm{UF}_{6}$ supply for India's BWRs is cut off, they may fuel with $\mathrm{UO}_{2}-\mathrm{PuO}_{2}$.

Waste Management Strategy: Vitrification of HLW, interim storage for at least 20 years and geologic disposal in a crystalline rock formation; disposal of LLW and short-lived ILW in near-surface engineered facilities; disposal of long-lived ILW will be in a deep geological repository.

Cumulative Spent Fuel

Arisings (LWR/HWR)

Cumulative Waste Arisings

Primary solid wastes

LLW concentrates

ILW

HLW
1990

2000

\begin{tabular}{cr}
\multicolumn{1}{c}{1982} & 2000 \\
$1,700 \mathrm{~m}^{3}$ & $107,000 \mathrm{~m}^{3}$ \\
$2,500 \mathrm{~m}^{3}$ & $77,000 \mathrm{~m}^{3}$ \\
$650 \mathrm{~m}^{3}$ & $20,000 \mathrm{~m}^{3}$ \\
$350 \mathrm{~m}^{3}$ & $8,000 \mathrm{~m}^{3}$
\end{tabular}

\section{Industrlal-Scale Activities}

- Heavy-water design capacity (t/a): 1993

- Uranium mining and milling (t/a): 1985

- $\mathrm{UO}_{2}$ fuel fabrication (t/a):

1984

210

2000

1,500

- Fuel reprocessing ( $\mathrm{t} / \mathrm{a})$;

Trombay pilot plant

Tarapur plant

1962

Kalpakkam plant

$1992 / 93$

- HLW vitrification: Tarapur (1985)

\section{Major Milestones}

- Interim Storage Plant - Tarapur

- Interim Storage/Waste Immobilization Plant - Kalpakkam 


\section{INTERNATIONAL RELATIONSHIPS}

Member of IAEA. Agreement with U.S. on peaceful nuclear cooperation.

India has not signed the NPT and has generally resisted the imposition of safeguards by individual suppliers (this has led to difficulties with supply of enriched uranium, reactor equipment, and heavy water).

India has agreements with several conntries on various aspects of the nuclear fuel cycle. Among them, signed in mid-1990, agreemenis with Vietnam (pilot plant for monazite processing snpplied by India) and Cuba (Cuban scientists being trained in nuclear power generation in India) for expanded cooperation in nuclear energy. 
INDIA

PNL-9450-1

\section{ORGANIZATION}

Prime Minister

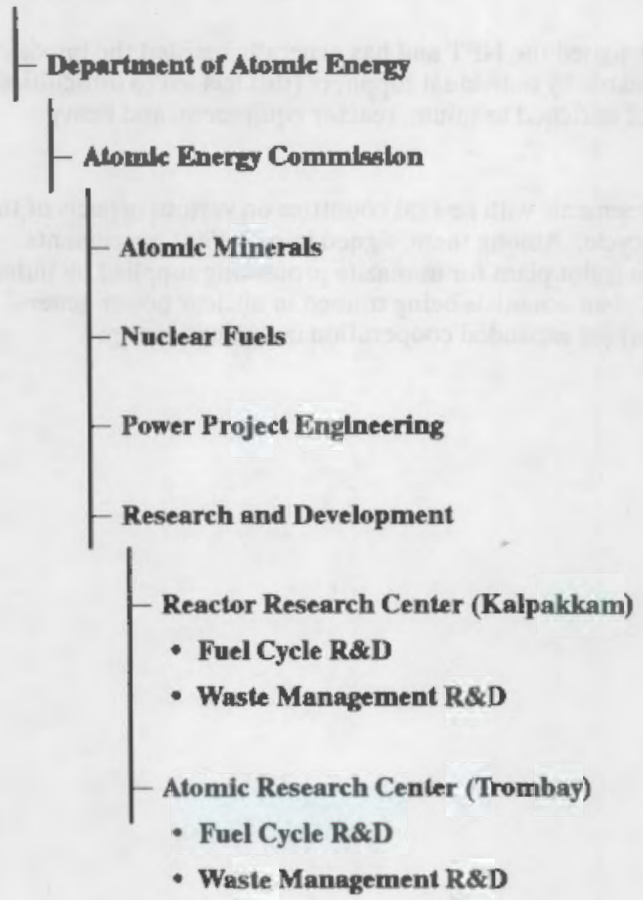




\section{$\underline{\text { BARC }}$}

Bhabha Atomic Research

Centre, Trombay

Bombay 400085 , India

Director

Director, Nuclear Safety Group

Waste Management Division

Ceutral. WM Facil., Kalpakkam

Radiol. Protection Division

Director, Chem. Engineering Group
Tel: $\quad 91-22-551-4910$ or 91-22-551-8700

Fax: $\quad 91-22-556-0750$

Dr. A. N. Prasad

V. N. Meckoni

M. T. Samuel

R. V. Amalraj

K. G. Vohra

B. K. Garg

Activities: BARC has five test reactors; radiochemistry and isotope laboratories; an isotope production and processing unit; pilot plants for production of heavy water, zirconium, and titanium; a thorium plaut; a uranium metal plant; a pilot-scale fuel reprocessing plant; the Fuel Irradiation and Processing Laboratory; and supporting facilities. Fuel cycle R\&D includes fuel reprocessing; HLW solidification; treatment of alpha-emitting wastes (incineration, wet oxidation, decontamination, and immobilization of cladding hulls); D\&D; and waste isolation in geologic formations.

\section{Facilities}

- Trombay Fuel Reprocessing Plant

Mission: Reprocess natural uranium metal fuels.

Design Basis: Chemical declad, PUREX flowsheet; contact maintenance; capacity, 0.1-0.15 tHM/d.

History: On-line, 1965-1974; modified and being readied to operate again.

- WIP (Waste Immobillzation Plant) - Trombay

Startup: construction, 1981; hot operation, 1990.

- Experimental Uranium Enrlchment Facility 


\section{DAE}

Department of Atomic Energy

Chatrapati Shivaji Marharaj Marg

Bombay 400039 , India

Minister, Science/Technology

Atomic Energy Commission (AEC) Tel: $\quad$ 91-22-202-2543 or 91-22-202-6823

Fax: $\quad 91-22-204-8476$

Chairman

Dr. Rajagopala

Chidambaram

Secretary

K. V. Mahadeva Rao

Atomic Energy Regulation Board (AERB)

Chairman

A. Gopalakrishnan

Funetion: Regulation and licensing of nuclear facilities.

Nuclear Power Corporation (formerly Nuclear Power Board)

Function: Design, construction, and operation/maintenance of nuclear power stations; help realize nation's goal of having $\mathbf{1 0 , 0 0 0}$ MWe of nuclear power on-line by the year 2000 . 


\section{IGCAR}

Indira Ghandi Centre

for Atomic Research

Kalpakkam 603102

Tamil Nadu, India

Tlx: $\quad 041-6244$

Fast Breeder Reactor Centre

Dr. Placid Rodriguez

Located near Madras power station.

Function: Fuel cycle R\&D; FBR technology; reprocessing of FBR fuels.

Facilities

- Fast Breeder Test Reactor

- Kalpalkam Fuel Reprocessing Loboratory Mission: Develop and test equipment and unit operations for FBR fuel reprocessing.

\section{KOLAR WASTE DISPOSAL RESEARCH STATION}

Located in the Kolar gold mine area near Bangalore, Karnataka State.

Function: Assess the suitability of peninsular gneisses for location of a deep geologic repository (in-situ studies).

Description: Tunnel extended from abandoned section of one of the Kolar gold mines into a neighboring gneissic formation.

History: Startup, late 1979. 


\section{MAPS}

Madras Atomic Power Station

Kalpakkam, India

Function: Nuclear power production, fuel reprocessing, and waste treatment; plutonium fuel fabrication for FBRs.

\section{Facilities}

- Fuel Reprocessing Plant Kalpakkam Mission: Reprocess spent fuel from the Kalpakkam reactors and from the 15-MW FBTR commissioned in 1985.

Design Basis: PUREX process, with a separate line for FBTR mixed-carbide fuels; capacity, originally $0.5 \mathrm{tHM} / \mathrm{d}$ for PHWR fuels, now increased to 200 tHM/a; cold operation, 1991.

- WIP (Waste Immobilization Plant)-Kalpakkam Construction startup, 1983; commissioning, 1993.

- ISF (Interim Storage Faclity)-Kalpakkam

\section{$\underline{\text { NFC }}$}

Nuclear Fuel Complex

Hyderabad, India

\section{Facilities}

- Fuel Fabrication Plant

Initial throughput of $50 \mathrm{t} / \mathrm{a}$ increased 1990 to $350 \mathrm{t} / \mathrm{a}$; expected to go to $600 \mathrm{~L} / \mathrm{a}$. 


\section{$\underline{\text { NSC }}$}

\section{Nuclear Science Center}

New Delhi, India

Function: Established through the University Grants Commission to encourage nuclear research outside of government-sponsored work. The facility below is only available to university researchers.

\section{Facilities}

\section{- Pelletron Accelernlor Facllity} Commissioned earty in 1991 . Housed in 100-foot-high lower, can accelerate atoms up to $16 \mathrm{MeV}$.

\section{TAPS}

Tarapur Atomic Power Station

Tarapur, Maharashtra, India

Function: Provide electric power, reprocess spent fuel from Tarapur reactors, and immobilize the associated wastes.

\section{Facilities}

- PREFRE (Fuel Reprocessing Plant) - Tarapur Mission: Reprocess natural and low-enriched $\mathrm{UO}_{2}$ fuels.

Design Basis: Chop-leach head-end; PUREX flowsheet; contact maintenance; capacity, 150 tHM/a.

History: Construction completed, 1975; hot operation, 12/1982.

- WIP (Waste Immobilization Plant) Mission: Vitrify Tarapur HLW.

Design Basis: Two-step calcination and melting in drainable pot; capacity, 25 liters/h HLLW, 125 kg glass/canister, 1 canister/d; product, borosilicate glass blocks.

History: Construction completed, 1981; hot startup, 1990.

(contd next page) 


\section{TAPS (contd)}

- SSSF (Solid Storage Surveillance Facility)

Mission: Provide air-cooled storage for WIP products.

Design Basls: Stack-induced natural draft air cooling; capacity for 20 years' storage of vitrified HLW from Tarapur and Trombay. History: Completion, 1990.

- ILW Bituminization Plant

- Polymerization Facility

- Pllot (hot cell-sized) Mox Fuel Fabrication Facility (1990) 
ITALY

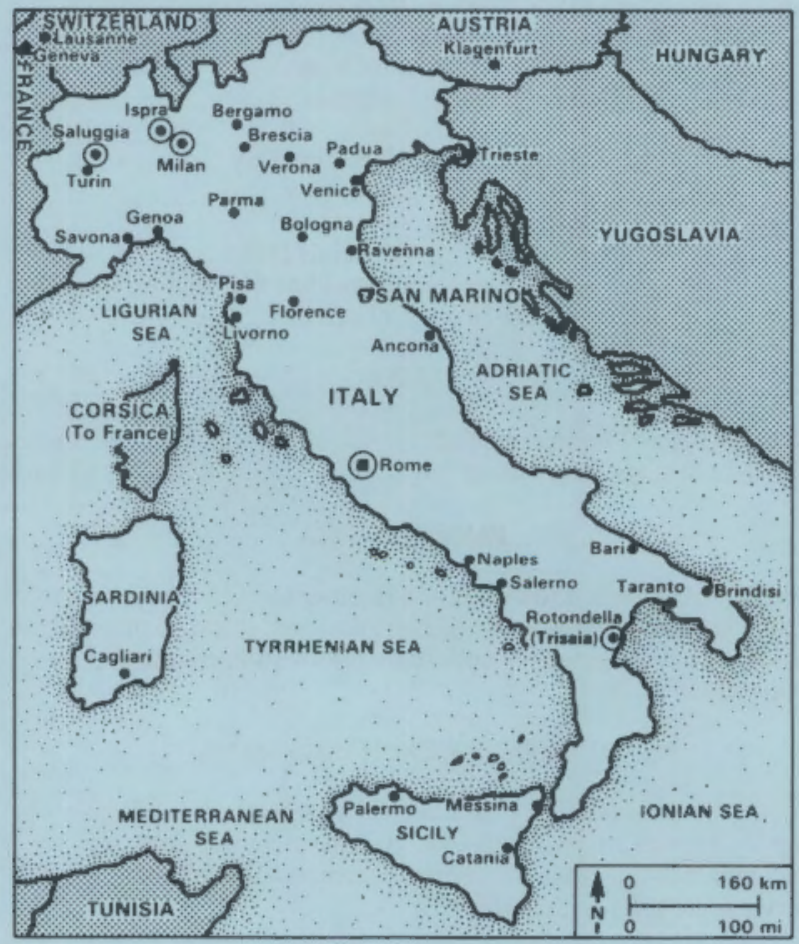




\section{ITALY}

\section{MAJOR PUBLIC HOLIDAYS (1994)}

$\begin{array}{lrl}\text { Jan } & 1 & \text { New Year } \\ \text { Jan } & 6 & \text { Epiphany } \\ \text { Apr } & 4 & \text { Easter } \\ \text { Apr } & 25 & \text { Liberation Day } \\ \text { May } & 1 & \text { Labor Day } \\ \text { Aug } & 15 & \text { Assumption } \\ \text { Nov } & 1 & \text { All Saints Day } \\ \text { Dec } & 8 & \text { Immaculate Conception } \\ \text { Dec 25-26 } & \text { Christmas }\end{array}$

\section{TIME}

Standard Time Washington, D.C.

Daylight Savings Time Period:

\section{PASSPORT/VISA}

A passport is needed to depart and re-enter the U.S. A visa is currently not required for a visit to Italy; however, it is recommended to consult a travel agency for up-to-date information concerning requirements.

\section{CURRENCY EXCHANGE RATE}

1 U.S. $S=1688.96$ Lira

per Wall Street Joumal, 01/31/94. As rates fluctuate daily, it is recommended to obtain current rates from local banks or newspapers prior to departure.

\section{DIRECT DIALING}

Individual numbers for direct dial to Italy are complete as listed, after dialing international access code: 011 . Country code is 39 ; listed local numbers include city code.

\section{U.S. EMBASSY - ROME}

American Embassy

Via Veneto 119/A

00187 Rome

Italy

Tel: $\quad 39-6-4674-2275$

Fax: $\quad 39-6-4674-2663$

Science Counselor

Gregory J. Dunn 
Population $\quad 1992 \quad 58.0$ million

\section{ENERGY}

$\begin{array}{llrl}\text { Electric Power Capacity } & 1992 & 61.9 & \text { GWe } \\ & 1995 & 68.6 & \text { GWe } \\ & 2000 & 80.6 & \text { GWe } \\ \text { Electric Power Production } & 1992 & 222.7 & \text { TWh } \\ & & 52 \% & \text { oil } \\ 19 \% & \text { hydro } \\ & & 16 \% & \text { gas } \\ & & 11 \% & \text { coal } \\ & & \text { geothermal }\end{array}$

\section{NUCLEAR POWER}

Policy: The current national energy plan calls for abandonment of nuclear power and increased use of coal and natural gas for electricity generation; research into nuclear energy will continue but with a reduced $R \& D$ budget.

\section{INDUSTRIAL FUEL CYCLE}

Waste Management Strategy: Spent fuel from previous nuclear power plant operations is being reprocessed abroad; vitrified HLW will be returned, starting in 1995; canisters will be temporarily stored until a final repository is available (clay formations are being considered); dry storage on site is also presently considered. No site for disposal of LLW/LW has been selected.

Cumulative SF Arisings 1,353 tU GCR

\section{INTERNATIONAL RELATIONSHIPS}

Member of EC, LAEA, and OECD/NEA; CEC Joint Research Center establishment is located in Northern Italy at Ispra. 


\section{ORGANIZATION}

- ENEA (Agency for New Technologies, Energy and Environment)-safety and regulatory; nuclear R\&D (principally at Casaccia, Saluggia and Trisaia).

- ANPA (National Agency for Environmental Protection)-inspection/control and health/environment protection.

- ENI--government-owned oil and energy holding company.

- Nucleco--company jointly owned by ENEA/ENI; LLW/LWW management (except disposal).

- CIPE (Interministerial Committee for Economic Planning)-designated regions where nuclear plants were to be located.

- FN (Fabricazioni Nncleari)--fuel fabrication/development.

- ENEL--state-owned power utility.

ENEA (Agency for New

Technologies, Energy \& Environment)

Ente per le Nuove Tecnologie,

l'Energia e l'Ambiente

Viale Regina Margherita 125

00198 Rome, Italy

Tel: $\quad 39-6-8528-1$

Fax: $\quad 39-6-8528-2591$

President

Director General

Prof. Nicolo Caribbo

Director, Energy Dept.

Dr. Fabio Pistella

Director, Fusion

Dr. Sergio Garribba

Dir., D\&D/WM

Dr. Roberto Andreani

Asst. Dir., SF/WM

Dr. Franco Pozzi

Dr. Piero Risoluti

(contd next page) 


\section{ENEA (contd)}

Function: Direct basic and applied research on energy and environment (mostly non-nuclear). Current nuclear-related work includes cooperation in international programs and is carried out in three departments: Fusion, Innovative Reactors, and Fuel Cycle Plant Dismantling.

Activities - Dismantling: Decommission facilities, including removal of stored nuclear material. Tasks: conditioning of liquid/solid radioactive wastes stored at the Eurex (Saluggia) and Itrec (Trisaia) plants and the Casaccia Center; removal (foreign reprocessing being considered) of spent fuel from reprocessing pilot plants; decontarnination and dismantling of plants and laboratories, including plutonium oxide fuel fabrication laboratory.

Owner: Government.

\section{ENEL (Nationai Electric Energy Agency)}

Ente Nazionale per

l'Energia Elettrica

Casella Postale 386

Via Giovan Battista Martini $3 \quad$ Tel: $\quad 39-6-8509-1$

00198 Rome, Italy Fax: $\quad 39-6-8509-3370$

$\begin{array}{ll}\text { Chairman } & \text { Franzo Viezoli }\end{array}$

Government agency, responsible for all electric power production. 


\section{FN}

Fabricazioni Nucleari

P.O. $B \times 16$

15062 Bosco Marengo (AL)

Italy

Tel: $\quad 39-131-7571$

Fax: $\quad 39-131-757250$

Chairman

Dr. P. Venditti

Function: Fabrication and development of special oxide nuclear fuels and special ceramic materials.

Owner: ENEA (95\%); AGIP, Fiat (5\%)

\section{NUCLECO}

Nucleco

Via Anguillarese 351

00060 Rome, Italy

Tel: $\quad 39-6-3046-302$

Fax: $\quad 39-6-3048-3081$

Chairman

Dr. P. Venditti

Function: Treat and dispose of LLW/LLW from hospitals, laboratories, industrial establishments, and nuclear plants; eventual plans include decommissioning work on nuclear installations.

Owner: ENEA (40\%); AGIP (60\%). 


\section{JAPAN}

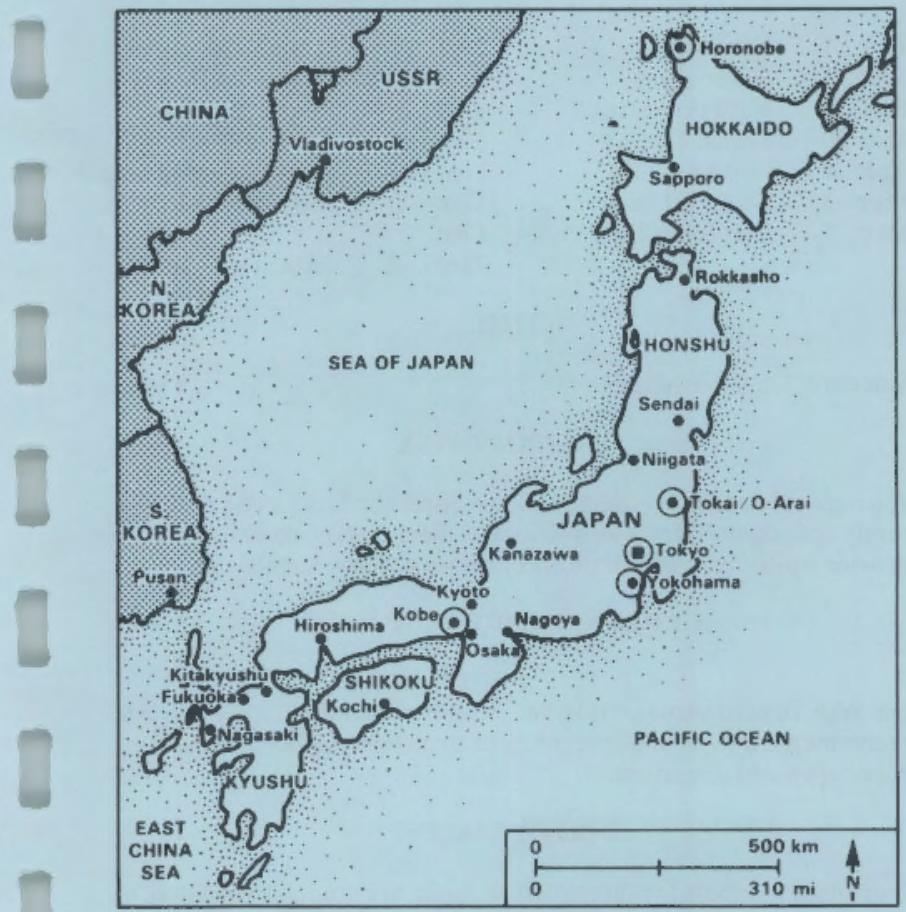


JAPAN

MAJOR PUBLC HOLIDAYS (1994)

$\begin{array}{lrl}\text { Jan } 1 & \text { New Year } \\ \text { Jan } 15 & \text { Adult's Day } \\ \text { Feb } 11 & \text { Nat'l Foundation } \\ \text { Mar } 20 & \text { Vernal Equinox } \\ \text { Apr } 29 & \text { Greenery Day } \\ \text { May } 3 & \text { Constitution } \\ \text { May } & 5 & \text { Children's Day }\end{array}$

Jan 1 New Year

Jan 15 Adult's Day

Feb 11 Nat'l Foundation

Mar 20

May 5
Sep 15 Respect for Aged

Sep 23 Autumnal Equinox

Oct 10 Sports Day

Nov 3 Culture Day

Nov 23 Labor Thanksgiving

Dec 23 Emperor's Birthday

Dec 29-

Jan 3 Govt. Off Season

\section{TIME}

Standard Time Washington, D.C.

+14 hours

\section{PASSPORT/VISA}

A passport is needed to depart and re-enter the U.S.; a visa is currently not required for a visit to Japan. Most travel agencies can provide up-to-date information concerning requirements.

\section{CURRENCY EXCHANGE RATE}

1 U.S. $\$=108.48$ Yen

per Wall Street Journal, 01/31/94. Because rates fluctuate daily, it is recommended to obtain current rates from local banks or newspapers prior to departure.

\section{DIRECT DIALING}

Individual numbers for direct dial to Japan are complete as listed, after dialing international access code: 011. Country code is 81; listed local numbers include city code.

\section{U.S. EMBASSY - TOKYO}

American Embassy

10-1, Alcasaka 1-chome, Minato-ku Tokyo 107, Japan

Tel: $\quad 81-3-3224-5000$

Fax: 81-3-3505-1862

Science Minister-Counselor

Michael A. Michaud

Tel: $\quad 81-3-4334-5500$

Fax: $\quad 81-3-3224-5229$

DOE Representative

Milton A. Eaton

Tel: $\quad 81-3-3224-5444 / 78$

Fax: $\quad 81-3-3224-5769$ 
Population

1992126 million

ENERGY

$\begin{array}{lrrl}\text { Electric Power Capacity } & 1992 & 178 & \text { GWe } \\ & 19 \% & \text { nuclear } \\ & 1995 & 195 & \text { GWe } \\ & 20 \% & \text { nuclear } \\ & 2000 & 223 & \text { GWe } \\ & & 21 \% & \text { nuclear }\end{array}$

Electric Power Production

$\begin{array}{lrl}1992 & 888.3 & \text { TWh } \\ & 31 \% & \text { oil } \\ & 25 \% & \text { nuclear } \\ & 20 \% & \text { gas } \\ & 15 \% & \text { coal } \\ & 9 \% & \text { hydro } \\ 1995 & 31 \% & \text { nuclear } \\ 2000 & 35 \% & \text { nuclear }\end{array}$

\section{NUCLEAR POWER}

Policy: Strong nnclear power program to lessen dependence on foreign energy sources; install LWRs for near-term needs; develop advanced HWR (ATR); aim for commercial FBR operation 20202030; supply domestic needs and build export business.

Nuclear Power Capacity $\begin{array}{lll}1992 & 33.9 & \text { GWe } \\ 1995 & 39.6 & \text { GWe } \\ 2000 & 47.5 & \text { GWe }\end{array}$

Reactor Mix

1993 GCR 1 (1966)

HWR 1 (1979)

BWR 24 (1970-93)

4 (1994-97)

PWR 20 (1970-93)

3 (1994-97)

FBR 1 (1994)

Reactor Development

HWR (ATR), LMFBR, HTGR 


\section{INDUSTRIAL FUEL CYCLE}

Policy: Obtain ownership of foreign uranium resources; develop complete fuel cycle capability (enrichment, reprocessing, and waste treatment; buy foreign reprocessing services until domestic capacity is available); recycle Pu to FBRs, HWRs, and LWRs.

Waste Management Stralegy: HLW - vitrify with borosilicate glass, store for 30-50 years, and dispose in geological formations; LLW dispose in engineered structures in shallow-land facility and at sea, if politically feasible.

$\begin{array}{llr}\text { Cumulative SF Arisings (LWR) } & 1990 & 7,500 \mathrm{tU} \\ & 1995 & 12,400\end{array}$

Industrial-Scale Activities (Capacity)

- Uranium mining and conversion ( $\left.\mathrm{tUF}_{6} / \mathrm{a}\right):$

- Uranium reconversion (tU/a): $\quad 1,028$

- Uranium enrichment (tSWU/a): $1981 \quad 50$

$1988 \quad 250$

- Fuel fabrication

$2000 \quad 3,000$

- $\mathrm{UO}_{2}$ for LWR (tU/a): $\quad 1987$

2,495

- MOX for FBR (L/a):

1988

- MOX for ATR (t/a):

1988

1993

- Reprocessing (t/a): $\quad 1981$ 


\section{Major Milestones}

- Storage facility for vitrified HLW from COGEMA/BNFL 1995

- Fuel reprocessing plant (Rokkasho-mura) SF storage reprocessing operation

- Selection of demonstration site for in-situ test with HLW disposal package

- FBR fuel reprocessing pilot plant operation $\quad>2000$

- Startup of HLW disposal site

- Experimental ocean disposal of LLW

\section{INTERNATIONAL RELATIONSHIPS}

DOE/PNC Agreement for Cooperation in the Area of Radioactive Waste Management

Term: $12-3-86$ to $12-3-96$

Scope: HLW/TRU waste; waste form development, assay, and characterization; treatment/packaging/transportation; storage/disposal; D\&D; facility operations; environment/ safety and public acceptance issues. Emphasis on information exchange of HLW and TRU waste conditioning technology.

Member of IAEA and OECD/NEA; cooperative agreements with Australia (SYNROC development), Canada, China, France, U.K.

\section{ORGANIZATION}

Government fnnds nuclear R\&D and is responsible for HLW disposal; industry handles the commercial fuel cycle and LLW disposal and pays for HLW disposal; see next three pages for organizational relationships and responsibilities. 


\section{NUCLEAR FUEL CYCLE/WASTE MANAGEMENT ORGANIZATION}

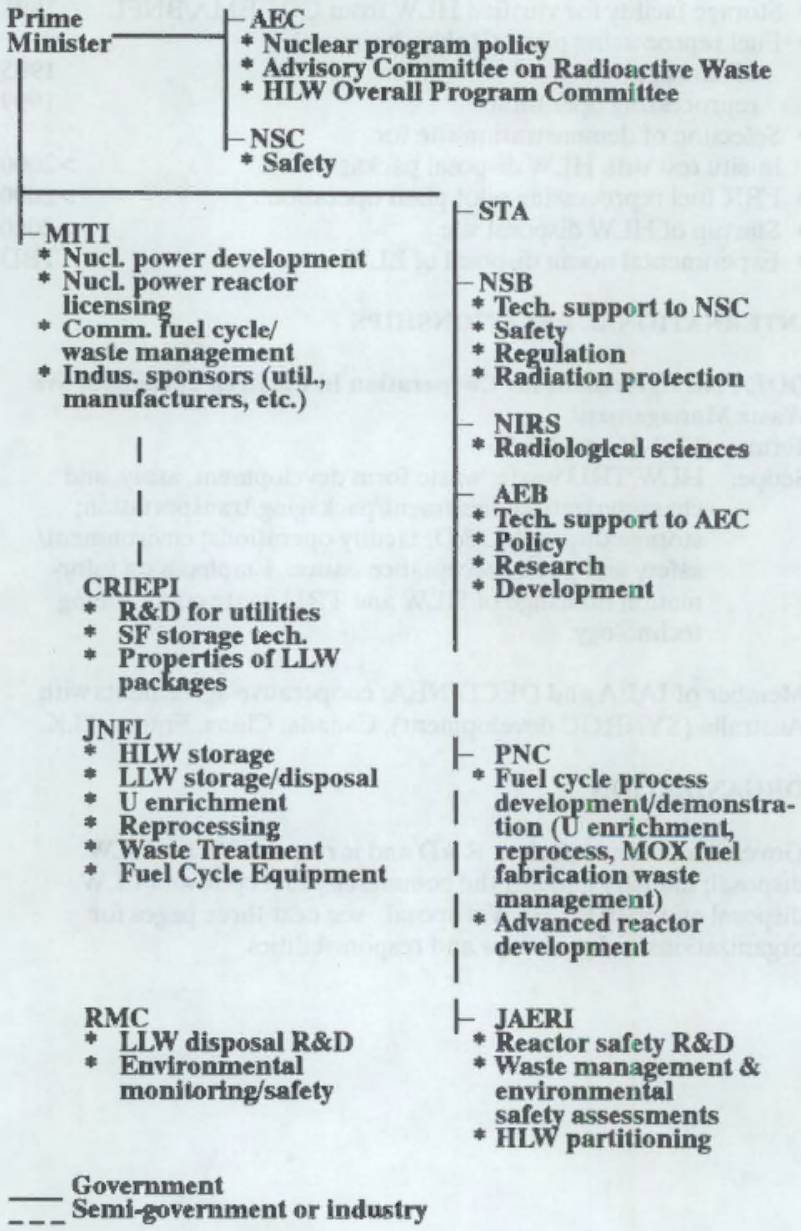




\section{PARTIAL PNC ORGANIZATION}

President-Board of Directors

$$
\begin{aligned}
& \text {-Technology Management Division } \\
& \text { - Policy Planning Division } \\
& \text { - Safety Division }
\end{aligned}
$$

-Nuclear Fuel Technology Development Div.

Plutonium Fuel Division

Reprocessing Technology Development Div.

- Waste Technology Development Division

- Nuclear Waste Treatment Division

Tokai Reprocessing Plant 


\section{PARTIAL JAERI ORGANIZATION}

President

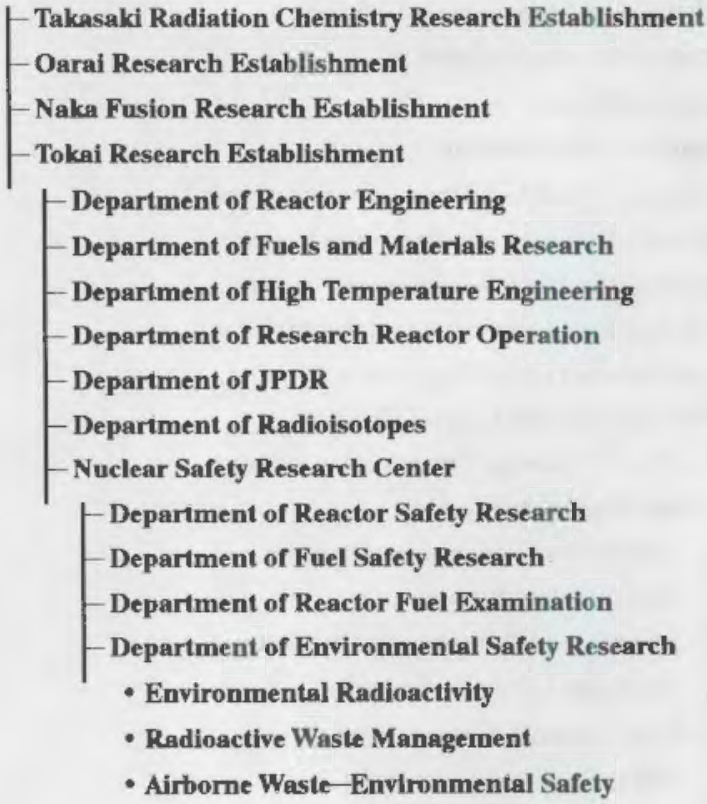


Atomic Energy Bureau

Science and Technology Agency

2-1 Kasumigaseki 2-chome

Chiyoda-ku, Tokyo 100, Japan

Director General

Deputy Director General

Director, Policy Div.

Dir., Power Reactor Dev. Div.

Dir., Nuclear Fuel Div.

Dir., Research/Internatl. Div.
Tel: $\quad 81-3-3581-1686$ or 81-3-3581-5271

Fax: $\quad 81-3-3592-1239$

Hiroto Ishida

Toshio Okazaki

Haruo Suzuki

Takaschi Kisaka

Yasutaka Moriguchi

Takayuki Shirao

Function: Provide support to the Atomic Energy Commission (AEC).

$\underline{\text { AEC }}$

Atomic Energy Commission

2-1 Kasumigaseki 2-chome

Chiyoda-ku, Tokyo 100

Japan

Tel: $\quad 81-3-3581-2585$ or

81-3-3581-5271

Fax: $\quad 81-3-3581-5198$

Chair (Minister of State

Satsuki Eda

for Science/Technology)

Acting Chair

Dr. Akira Oyama

Function: Formulate national policy on nuclear energy R\&D and utilization; advise Prime Minister.

\section{CRIEPI}

Central Research Institute

of Electric Power Industry

1-6-1, Ohtemachi

Chiyoda-ku, Tokyo 100, Japan

Tel: $\quad$ 81-3-3201-6601

Fax: $\quad$ 81-3-3287-2880

President

Susumu Yoda

(contd next page) 


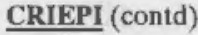

Function: Provide R\&D support for utilities.

Waste Management R\&D: Transportation, storage, disposal of LLW; intermediate and long-term storage of spent fuel; long-term storage and disposal of HLW.

Energy and Environmental

Research Laboratory for

Energy and Electric Power

2-11-1, Iwato-kita

Komae-shi, Tokyo 201, Japan

Tel: $\quad 81-3-3480-2111$

Fax: $\quad 81-3-3488-6697$

Function: Laboratory under CRIEPI.

\section{GIRIO}

Government Industrial Research

Institute, Osaka

1-8-31 Midorigaoka, Ikeda-shi $\quad$ Tel: $\quad$ 81-727-51-8351

Osaka 563, Japan

Fax: $\quad 81-727-51-6945$

Director, 4th Department

Nuclear Waste Program

Dr. Ryozo Hayami

Dr. Ryohei Terai

Waste Management R\&D: Alternatives for HLW solidification; waste form characterization.

\section{HITACHI}

Hilachi, Ltd.

6, Kanda-surugadai, 4-chome

Tel: $\quad 81-3-3258-1111$

Chiyoda-ku, Tokyo 101, Japan

Fax: $\quad 81-3-3258-6218$

President

Tsutomu Kanai

Gen. Mgr., Nuc. Power Systems Div. Tsutomu Hayashi

Sr. Chief Engineer

Atou Shimozato

Nuclear Power Development

Ryoichi Kondo

(contd next page) 


\section{HITACHI (contd)}

Waste Management R\&D: Development of volume reduction systems for radioactive waste; application of automation and robotics technology; development of advanced control technology through use of fiber optics.

Hitachi Engineering Co,, Ltd.

2-1 Saiwai-cho, 3-chome

Hitachi-shi, Ibaraki-ken, 317

Japan

Tel: $\quad$ 81-294-24-1111

Fax: $\quad 81-294-22-8987$

President

Nuc. Power Plant Construction

Akira Sakai

Nuc. Fuel Project

Nuc. Fuel Cycle Project

Kiyoshi Shimizu

Yasuo Hirose

Sadatoshi Inoue

Waste Management R\&D: Develop technology to reprocess spent LWR fuel; fixation, storage, and disposal of HLW; spent fuel storage; $\mathrm{Pu}$ fuel production; decommissioning.

\section{$\underline{\text { IHI }}$}

Ishikawajima-Harima

Heavy Industries Co., Ltd.

Shin-Ohtemachi Bldg.

2-1, Ohtemachi 2-chome

Chiyoda-ku, Tokyo 100, Japan

Tel: $\quad 81-3-3244-5111$

Fax: $\quad 81-3-3286-2440$

President

Executive Vice President

Kousaku Inaba

Gen. Mgr., Nucl. Power Sales

Makio Amano

Hiroshi Tomioka

\section{IHI Research Institute}

Yokohama Branch

1, Shin-nakaharacho, Isogo-ku

Yokohama 235, Japan

Tel:

81-45-751-1231

Fax: $\quad 81-45-753-9564$

Wasle Management R\&D: Development of nuciear waste management system. 


\section{JAERI}

Japan Atomic Energy

Research Institute

2-2, Uchisaiwai-cho, 2-chome

Chiyoda-ku, Tokyo 100

Japan

Tel: $\quad 81-3-3592-2111$

Fax: $\quad 81-3-3580-6107$

President

Vice President

Vice President

Exec. Director, International

Shozo Shimomura

Masaji Yoshikawa

Eiichi Tsuji

Hirofumi Satake

Location: JAERI headquarters and Radioisotope Center are in Tokyo; the Tokai and Oarai research establishments share government reservations at Tokai-mura and Oarai-machi with PNC; Tokgi and Oarai are 120 and $100 \mathrm{~km}$, respectively, northeast of Tokyo, near the ocean; these sites can be reached by train from Tokyo to the ciry of Mito, then by taxi; the Naka Research Establishment (fusion energy) is in Naka-machi near Tokai-mura.

Function: Semi-governmental research organization implementing national long-term programs in nnclear energy, including joint projects and international cooperation.

\section{JAERI: OARAI}

Japan Atomic Energy Research Institute

Oarai Research Establishment

Oarai-machi

Higashi-Ibaraki-gun

Ibaraki-ken Pref. 311-13, Japan

Tel: $\quad 81-292-67-4111$

Fax: $\quad 81-292-66-2235$

Director General

Yoshihiko Kaneko 


\section{JAERI: TOKAI}

Japan Atomic Energy Research Institute

Tokai Research Establishment

Tokai-mura, Naka-gun

Ibaraki-ken Pref. 319-11

Tel: $\quad 81-292-82-5111$

Japan

Fax: $\quad 81-292-82-0528$

Director General

Deputy Director General

Deputy Director General

Deputy Director General

Shojiro Matsuura

Hiroyuki Takahashi

Michio Ichikawa

Tatsuo Kondo

\section{Facilities}

- WASTEF (glove box and hot cell facilities) Mission: HLW safety evaluations.

History: Cold Startup: 1981; Hot, 1982.

- STEM (Simulation Test for Environmental Radionuclide Migration)

Mission: Safety evaluation for land disposal of LLW. History: Startup, 1983.

\section{- NUCEF}

Facility to conduct research on safety of SF reprocessing and treatment of radioactive wastes to support licensing review by STA on reprocessing plant being constructed by JNFL.

STACY (Static Experimental Critical Facility)

TRACY (Transient Experimental Critical Facility)

Experimentel Facility for TRU (research on separation/recovery of TRU) 


\section{JGC}

JGC Corporation

Nuclear and Advanced Technology

New Ohtemachi Bldg.

2-1 Ohtemachi 2-chome

Chiyoda-ku, Tokyo 100, Japan

Tel: $\quad 81-3-3279-5441$

Fax: $\quad 81-3-3273-8050$

President

Dr. Eiji Watanabe

Exec. Vice President

Dr. Takao Nakajima

Genera! Manager, Director

Deputy General Manager

Dr. Hiroshi Kuribayashi

Keisuke Okazakj

Function: Design and construction of fuel reprocessing and radwaste treatment facilities.

JGC Nuclear Research Center

2205 Narita-cho, Oarai-machi

Higashi-Ibaraki-gun

Ibaraki Pref. 311-13

Japan

Tel: $\quad 81-292-66-3311$

Fax: $\quad 81-292-66-8810$

Nuc. \& Adv. Tech. Projects

Yasuhiro Moriyı

Wasle Management R\&D: Wet oxidation (organic materials, e.g., spent ion exchanger resin) incinerator; waste solidification processes (cementing, bituminization, plastic solidification); regeneration waste recycle process; selective nuclide removal process; ash melting process.

\section{Facilities}

- Demonstration Incineration Plant

Mission: Simultancously melt combustible and noncombustible wastes.

Design Besis: $100 \mathrm{~kg} / \mathrm{h}$ at $1500^{\circ} \mathrm{C}$. LLW combustion technology licensed from Belgonucleaire SA.

(contd next page) 


\section{$\underline{\text { JGC (contd) }}$}

- Contaminated Liquid Waste Recycle Plant

Mission: Recovery of clean water from LLLW for re-use.

Design Basis: 75 liter/min.; filtration; reverse osmosis; active carbon bed adsorption; chelate resin adsorption; ion-exchange adsorption; evaporation.

\section{JNFL}

Japan Nuclear Fuel Limited

1-12-15 Honcho, Aomori-shi

Aomori 030, Japan

Tel:

Fax: $\quad 81-177-31-1551$

President

Vice Presidents

Kiyoshi Nozawa

Tetsuo Hirasawa

Kiyoshi Fuseya

Hiroshi Takashina

Hideto Kamekawa

Function: Construct/operate facilities for uraninm enrichment, fuel reprocessing, and LLW dlsposel in the Oishitai area of Rokkasho-mura.

Owner: Japanese utilities (10).

JNFL Tokyo Branch Orice

Daiichi Seimei Bldg.

2-10 Hirakawa-cho 1-chome

Chiyoda-ku, Tokyo, Japan

Tel: $\quad 81-3-3239-6521$

Fax: $\quad 81-3-3239-6479$

Rokkasho Works Construction Office

504-22 Aza Notsuke, Oaza Obuchi

Rokkasho-mura, Kamikita-gun

Tel: $\quad 81-175-72-3311$

Aomori-ken, Japan

Fax: $\quad 81-175-72-3228$

Managing Director/Gen. Manager Yuuta Suzuki

(contd next page) 


\section{JNFL (contd)}

\section{Facilities}

- Uranium Enrichment Plant (at Oishitai, Rokkasho-mura). Mission: Enrich uranium for Japanese utilities to establish indigenous nuclear fuel cycle (cost: U.S. \$1.38 billion). Capacity: 150 tSWU/a initially; 1500 tSWU/a final capacity. History: Initial startup, 1992; 1500 tSWU/a $\sim 2000$.

- LLW Disposal Facillty (at Oishitai, Rokkasho-mura). Mission: Dispose of Japanese utilities-generated LLW (cost: U.S. $\$ 1.23$ billion).

Capacity: Approximately 1 million drums initially, final capacity equivalent to 3 million drums.

History: Startup, 12/1992.

- Fuel Reprocessing Plant (at Iyasakatai, Kamikita-gun, Rokkasho-mura).

Mlssion: Reprocess Japanese fuels.

Design Basis: 800 tHM/a; 3000 tU storage pool; HLW vitrification/storage, partial design by SGN, France, construction start 1992, operations 1995.

Milestone: SF storage, 1995; FRP startup, 1999.

\section{KOBE STEEL}

Kobe Steel, Ltd.

No. 3-18, Wakinohamacho

1-chome

Chuoh-ku, Kobe 651, Japan

Fax: $\quad 81-78-251-1551$

Fax: $\quad 81-78-232-3459$

General Manager

Toru Abe

Mech. Eng. Research Lab. (MERL) Thkao Mizguchi

Nuclear Engineering

Fumiaki Komatsu

Function: Manufacture SF transportation/storage casks; waste treatment equipment/systems; LLW/HLW handling/storage. 


\section{MITI}

Ministry of International

Trade and Industry

3-1, Kasumigaseki 1-chome

Chiyoda-ku, Tokyo 100, Japan

Minister

V. Minister

International Affairs
Tel: $\quad$ 81-3-3501-1511

Fax: $\quad 81-3-3501-0643$ or 81-3-3501-0644

Hiroshi Kumagai

Hideaki Kumano

Sozaburo Okamatsu

\section{MITL/ANRE}

Agency of Natural Resources and Energy

Ministry of International

Trade and Industry

3-1, Kasumigaseki 1-chome

Chiyoda-ku, Tokyo 100, Japan

Tel: $\quad$ 81-3-3501-1511

Fax: $\quad 81-3-3501-0643$ or 81-3-3501-0644

Director-General

Dep. Director-General

Dep. Dir.-Gen., Nucl. Energy

Dir., Nuclear Industry

Dir., Internatl. Nnc. Affairs

Tomio Tsutsumi

Yasuo Hayashi

Tohru Namiki

Hideo Matsui

Reiji Nagase

\section{$\underline{\text { MMC }}$}

Milsubishi Materials Corporation

5-2 Ohtemachi 1-chome

Chiyoda-ku, Tokyo 100, Japan

Vice President

Gen. Mgr., Nuc. Energy

Gen. Mgr, Tech. Planning Dept.

Gen. Mgr., Nuc. Resources

Development/Waste Mgmt.
Tel: $\quad 81-3-3213-2111$

Fax: $\quad 81-3-3215-2435$

Dr. Yuumi Akimoto

Eiji Yagi

Dr. Tamolsu Ishii

Takaaki Kashiwagi

Waste Management R\&D: Design and research on facilities for spent fuel storage and reprocessing, waste treatment, and geologic disposal. 


\section{MOFA}

Ministry of Foreign Affairs

2-1 Kasumigaseki 2-chome

Chiyoda-ku, Tokyo 100, Japan

Tel: $\quad 81-3-3580-3311$

Fax: $\quad 81-3-3581-9470$

Minister

Director General, Disarmament/

Scientific Affairs

Director, Nuclear Energy

Deputy Director

Tsutomu Hata

Akira Hayashi

Yukiya Amano

Yoshifumi Okamura

\section{NIRS}

National Institute of

Radiological Sciences

9-1, Anagawa 4-chome

Chiba-shi, Chiba Pref. 260, Japan

Tel: $\quad 81-472-51-2111$

Fax: $\quad 81-472-56-8301$

Director General

Hiromichi Matsudaira

Function: Attached to the Science \& Technology Agency; responsible for carrying out studies on radiation hazards, applications for medical use, and education/training of engineers in these areas. 


\section{NSB}

Nuclear Safety Bureau

Science and Technology Agency

2-1, Kasumigaseki 2-chome

Chiyoda-ku, Tokyo 100, Japan

Tel: $\quad 81-3-3581-5271$

Fax: 81-3-3581-0774

Director-General

Deputy Director-General

Dir., Nuc. Mtls. Reg. Div.

Dir., Nuc. Safety Policy Div.

Dir., Reactor Reg. Div.

Dir., Safeguards Division

Dir., Radiation Protec. Div.

Dir., Nuc. Safety Policy Res.

Isamu Sasaya

Hisaharu Dosho

Itsuro Misumi

Kimihiko Oda

Mitsuo Hayashi

Kiyoshi Honma

Kaoru Naito

Haruo Suzuki

Function: Provide support to the Nuclear Safety Commission.

$\underline{\text { NSC }}$

Nuclear Safety Commission

2-1, Kasumigaseki 2-chome

Chiyoda-ku, Tokyo 100, Japan

Tel: $\quad 81-3-3581-5271$

Fax: $\quad 81-3-3581-0774$

Chairman

Yasumasa Togo

Function: Responsible for carrying out national policy for safety and security of nuclear energy, its utilization, and related R\&D; advisory body to the Prime Minister's office. 


\section{PNC}

Power Reactor and Nuclear Fuel

Development Corporation

Sankaido Building

1-9-13 Akasaka

Minato-ku, Tokyo 107, Japan

President

Exec. Vice Presidents

Exec. Dir., Nuc. Fuel/Reprocess.

Exec. Dir., WM

Exec. Deputy Directors, WM

Deputy Director, WM

Coordination

Conditioning Research

Isolat'n Syst. Research

International Project

Geoscience Research

Dir., Fuel Cycle Develop.

Dir., Fuel Cycle Engineering

Dir., International

Deputy Dir., International

International Cooperation

U.S. DOE Tech. Representative

PNC Washington Office:

Power Reactor and Nuclear Fuel

Development Corporation

Suite 715

2600 Virginia Avenue NW

Washington, DC 20037

Manager

Tel: $\quad 81-3-3586-3311$

Fax: $\quad 81-3-3583-6386$

Takao Ishiwatari

Mitsuo Taguchi, Hiroshi

Ohishi

Hiroyoshi Kurihara

Masao Yamamoto

Yoshiro Asakura, Dr. Aiji

Yamato

Kouichi Tasurumaki

Tomohiro Asami

Yasumasa Ando

Dr. Noriaki Sasaki

Hideki Sakuma

Dr. Minoru Yamakawa

Hidechiyo Kashihara

Naomi Tsunoda

Tadatomo Yamaguchi

Takso Yagi

Dr. Takashi Kano

Jim Scott

81-3-3586-3311

Tel: $\quad$ 202-338-3770

Fax: $\quad 202-333-1097$

Masayori Tsutsumi 


\section{PNC: OARAI}

PNC Oarai Engineering Center

Oarai-machi, Higashi Ibaraki-gun Ibaraki Pref. 311-13, Japan

Tel: $\quad 81-292-67-4141$

Fax: $\quad 81-292-67-7147$

Director

Gen. Mgr., Waste Management

Director, Fuels/Materials

Kiminori Shiba

Yuji Enokido

Masami Katsuragawa

\section{Facilities}

- Incinerator

Mission: Burn solid LLW.

Design Basis: Three chambers--pyrolysis, combustion, afterburning.

- WDF (Waste Dismantling Facility)

Mission: Condition large contaminated equipment; develop D\&D technology.

Design Basis: Capacity to condition $5.5 \mathrm{t} / \mathrm{a}$.

History: Hot stariup, 1984.

\section{PNC: TOKAI}

PNC Tokai Works

4-33 Muramatsu

Tokai-mura, Naka-gun

Tel: $\quad 81-292-82-1111$

Ibaraki-ken 319-11, Japan

Fax: $\quad 81-292-82-1469$ or 81-292-82-9398

(contd next page) 
PNC: TOKAI (contd)

Director

Deputy Directors

Dir., Reprocessing Plant

Dir., Technology Dev. Coord'n

Dir., Health/Safety

Dir., Waste Technology Devel.

HLW Technology

LLW Technology

Geological Isolation Tech.

Dir., Waste Plants Operation

Dir., Reprocess. Tech. Devel.

Dir., Nuc. Fuel Tech. Devel.
Kenji Miyahara

Nobuyuki Sasao

Kei Nakata, Yasumasa Oki

Osamu Yamamura

Hisataka Ando

Naoyuki Sakurai

Sumio Masuda

Jin Ouchi

Yoshio Nakanishi

Tadashi Mano

Takao Akiyama

Koichi Onichi

Yoichiro Kishimoto

\section{Facilities}

\section{- Fuel Reprocessing Plant}

Mission: Reprocess low-enriched $\mathrm{UO}_{2}$.

Design Basis: Oxide fuels: chop-leach head-end; PUREX

flowsheet; capacity, $0.7 \mathrm{tHM} / \mathrm{d}$; remote maintenance of chop-leach equipment; contact maintenance of other components.

History: Startup, 09/77; 509 tU spent fuel processed through $12 / 90$.

- Tokai Plutonium Conversion Development Facility

Mission: Demonstrate PNC microwave process for co-conversion production of MOX.

Design Basls: $10 \mathrm{~kg} / \mathrm{d} \mathrm{MOX}\left(50 \% \mathrm{PuO}_{2}, 50 \% \mathrm{UO}_{2}\right)$.

History: Startup of hot operation, 10/83.

\section{- Tokai Plutonium Fuel Fabrication Facility}

Mission: Fabricate FBR and ATR fuels.

Design Basis: FBR fuels--1 t/a $\left(30 \% \mathrm{PuO}_{2}\right.$ in enriched $\left.\mathrm{UO}_{2}\right)$;

ATR fuels-- $10 \mathrm{t} / \mathrm{a}\left(2 \% \mathrm{PuO}_{2}\right.$ in $\left.\mathrm{UO}_{2}\right)$.

Throughput: Since 1979, 100 t MOX produced through 05/89.

(contd next page) 


\section{PNC: TOKAI (contd)}

- Toked Plutontum Fuel Production Facility Mission: Fabricate large quantifies of MOX fuel for FBR and ATR.

Design Basls: FBR fuels, 5 t/a; ATR fuels 40 t/a. Hislory: Startup of hot operation, 04/88.

- EDF (Engineering Demonstration Facility)

Mission: Nonradioactive, full-scale and/or engineering mockup tests of processes and equipment for FBR spent fuel reprocessing. History: Startup, 04/82.

- ETF (Engineering Test Facility)

Mlssion: Develop engineering test of HLW vitrification and ceramic meiter technologies.

Design Basis: Joule-heated melter.

History: Startup, 02/80.

- CPF (Chemical Processing Facility) - reprocessing and HLW treatment.

Mission: Radioactive studies of FBR spent fuel reprocessing and HLW solidification processes.

Design Basis: Five standard hot cells for breeder fuel reprocessing R\&D, five cells for waste conditioning R\&D; reprocessing, $1 \mathrm{~kg} /$ batch; HLW solidification, 10-liter/batch HLW. History: Hot tests, 09/82.

- KRF - Krypton Recovery Facility (pilot plant) Mission: Demonstrate ${ }^{85} \mathrm{Kr}$ recovery from Tokai-mura reprocessing plant off-gas.

Design Basis: Cryogenic distillation and pressurized cylinder storage.

History: Hot test, 03/88; operation, 04/88.

- Bitumization Demonstration Facillty

Mission: Immobilize LLLW concentrate.

Deslgn Basis: 200 liter/h.

(contd next page) 


\section{PNC: TOKAI (contd)}

- Incinerator

Mlssion: Burn solid LLW.

Design Basis: $600 \mathrm{~kg} / \mathrm{d}$.

- PWTF (Plutonium-Contaminated Waste Treatment Facility) Mission: Prepare PNC TRU wastes for disposal.

Design Basis: Incineration of combustibles/chloride-containing wastes; mechanical volume reduction.

History: Operation startup, 1987.

- PWSF (Plutonium-Contaminated Waste Storage Facility) Mission: Store PNC TRU waste.

Design Basis: 6000-drum capacity.

History: Operation startup, 1981.

- TVF (Tokai Vitriflcation Facility)

Mission: Vitrify and store HLW from the Tokai reprocessing plant; demonstrate technology.

Design Besis: Ceramic melter to produce a borosilicate glass; capacity, $0.35 \mathrm{~m}^{3}$ HLLW/d.

History: Construction start, 1988; cold test, 1992.

Milestone: Hot test, 1994.

- Recycle Equipment Test Facility

Mission: Demonstrate FBR fuel reprocessing equipment and process technolegy.

Design Basis: $10 \mathrm{~kg} / \mathrm{h}$

Milestone: Startup, 1994.

- FBR Fuel Reprocessing Pilot Plant (reprocessing and HLW treatment)

Mission: Demonstrate FBR fuel reprocessing and HLW solidification.

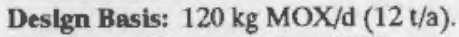

Milestone: Hot operation, 1997. 


\section{RWMC}

Radioactive Waste Management Center

Mori Building \#15

8-10, Toranomon 2-chome

Minato-ku, Tokyo 105, Japan

Tel: $\quad 81-3-3504-1081$

President

Fax: $\quad 81-3-3504-1297$

Executive Director

Tashio Fukuda

Takashi Tsusuki

Function: R\&D on safe and effective treatment and disposal techniques for radioactives wastes.

Owners: Japanese industry, MITI and STA.

STA

Science and Technology Agency

2-1 Kasumigaseki, 2-chome

Chiyoda-ku, Tokyo 100

Tel: $\quad 81-3-3581-5271$

Japan

Fax: 81-3-3592-1239

Minister, Science/Technology

Vice Minister

Deputy Minister

Director-General, AEB

Dep. Director-General, AEB

Dir., Policy Division, AEB

Director-General, NSB

Dep. Director-General, NSB

Satsuki Eda

Takuya Hirano

Kenichi Murakami

Hiroto Ishida

Toshio Okazaki

Haruo Suzuki

Isamu Sasaya

Shobu Kudo

Function: Established as an extra-ministerial agency of the Prime Minister's office for comprehensive administration and promotion of science and technology; the Atomic Energy Bureau (AEB) and the Nuclear Safety Bureau (NSB) are under STA jurisdiction; appropriate listings are under AEB and NSB, respectively. 


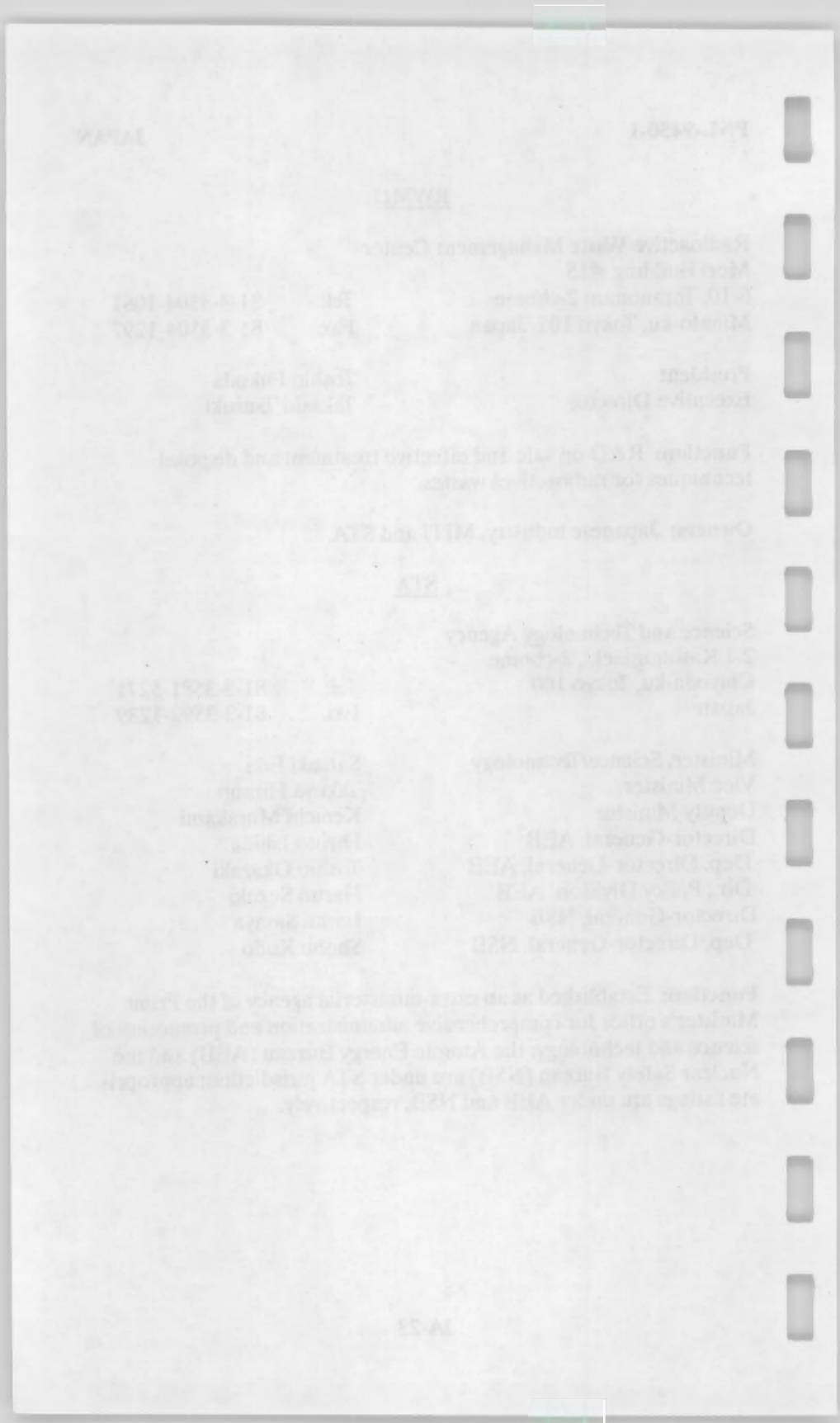




\section{KOREA (SOUTH)}

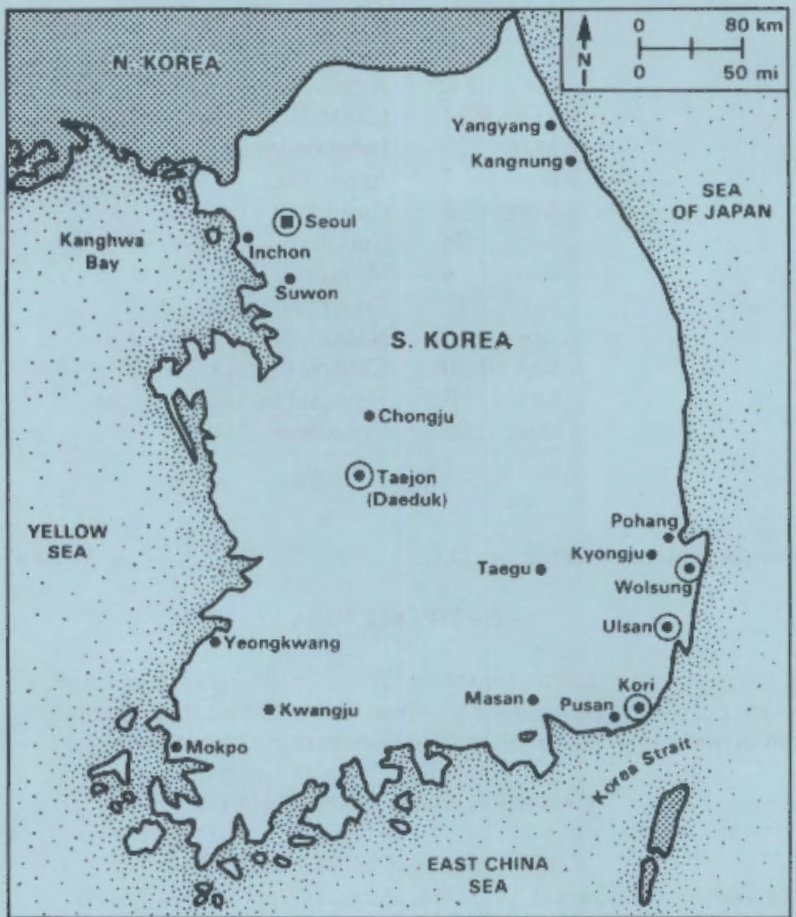


KOREA

\section{MAJOR PUBLIC HOLIDAYS (1994)}

$\begin{array}{lrl}\text { Jan } & 1-2 & \text { New Year } \\ \text { Feb } & 9-11 & \text { Lunar New Year } \\ \text { Mar } & 1 & \text { Independence } \\ \text { Apr } & 5 & \text { Arbor Day } \\ \text { May } & 5 & \text { Children's Day } \\ \text { May } & 24 & \text { Buddha's Birthday } \\ \text { Jun } & 6 & \text { Memorial Day } \\ \text { Jul } & 17 & \text { Constitution Day } \\ \text { Aug } & 15 & \text { National Day } \\ \text { Sep } 19-20 & \text { Chusok (Thanksgiving) } \\ \text { Oct } & 3 & \text { National Foundation Day } \\ \text { Dec } & 25 & \text { Christmas }\end{array}$

\section{TIME}

Standard Time Washington, D.C.

+14 hours

\section{PASSPORT/VISA}

A passport is needed to depart and re-enter the U.S.; in addition, a visa is currently required for a visit to Korea. Most travel agencies can provide up-to-date information concerning requirements.

\section{CURRENCY EXCHANGE RATE}

1 U.S. $\$=808.10$ Won $(W)$

per Wall Street Journal, 01/31/94. As rates fluctuate daily, it is recommended to obtain current rates from local banks or newspapers prior to departure.

\section{DIRECT DIALING}

Individual numbers for direct dial to Korea are complete as listed, after dialing international access code: $\mathbf{0 1 1}$. Country code is 82; listed local numbers include city code.

\section{U.S. EMBASSY - SEOUL}

American Embassy

82 Sejong-Ro, Chongro-Ku

Seoul, Republic of Korea

Science Counselor
Tel: $\quad 82-2-397-4114$

Fax: $\quad 82-2-738-8845$

F. Ken Crosher 
Population

1993

44 million

ENERGY

Electric Power Capacity

$\begin{array}{rrl}1993 & 27.7 & \text { GWe } \\ & 28 \% & \text { nuclear } \\ 1995 & 30.0 & \text { GWe } \\ & 29 \% & \text { nuclear } \\ 2000 & 32.0 & \text { GWe } \\ & 45 \% & \text { nuclear } \\ & & \\ 1993 & 144 & \text { TWh } \\ & 40 \% & \text { nuclear } \\ & -30 \% & \text { coal } \\ & -25 \% & \text { oil } \\ & 5 \% & \text { hydro } \\ 1995 & 40 \% & \text { nuclear } \\ 2000 & 40 \% & \text { nuclear }\end{array}$

\section{NUCLEAR POWER}

Policy: Continue expansion of electric power capacity; reduce dependence on foreign oil by strong nuclear program with indigenous manufacturing capability; long-term goal--develop FBR capability.

Nuclear Power Plant Capacity 1993 $7.6 \mathrm{GWe}$ 1995 2000 13.5 GWe

Reactor Mix 1993

$$
\begin{array}{ll}
\text { PWR } & 8(1978-89) \\
& 4(1995-99) \\
\text { HWR } & 1(1983) \\
& 1(1997)
\end{array}
$$

Reactor Development (feasibility studies) Advanced PWR

\section{INDUSTRIAL FUEL CYCLE}

Policy: Develop long-term contracts for fuel supplies, holdings of foreign uranium resources; fabricate fuel for PWR and HWR (CANDU); "wait and see" on reprocessing and recycle of $\mathrm{Pu}$ for FBR, CANDU, and LWR. 
Waste Management Strategy: LLW/ILW repository to be constructed by mid-1990s with emphasis on engineered barriers; candidate sites have been identified, but final decision is pending; utility surcharge of $2 \mathrm{mil} / \mathrm{kWh}$ to fund waste management; extended storage ( $~ 60$ years) of SF planned, in AR and AFR facilities; no decision has been made on reprocessing or disposal of SF/HLW.

Cumulative SF Arisings

$\begin{array}{ll}1990 & 1,500 \text { tU } \\ 1995 & 2,600 \text { tU } \\ 2000 & 4,400 \text { tU }\end{array}$

\section{Industrial-Scale Activities}

- Uranium milling--3t ore/d pilot plant.

- Uranium conversion, yellowcake to $\mathrm{UO}_{2}-100 \mathrm{tU} / \mathrm{a}$.

- $\mathrm{UO}_{2}$ fuel fabrication pilot plant--10 tU/a.

- $\mathrm{UO}_{2}$ fuel fabrication--200 tU/a. Startup, 1989.

\section{Major Milestones}

- LLW disposal site ( 500,000 drums)

\section{INTERNATIONAL RELATIONSHIPS}

Member of LAEA; agreement with U.S. for peaceful nuclear cooperation. 


\section{ORGANIZATION}

Deputy Prime Minister -.-. Atomic Energy Commission (AEC)

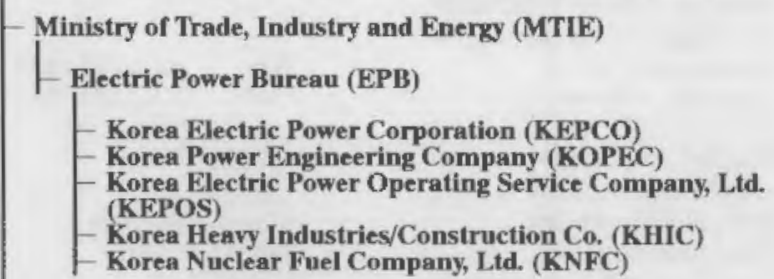

- Ministry of Science and Technology (MOST)

- Atomic Energy Omice (AEO)

Nuclear Policy Omce (NPO)

- Nuclear Policy Division

- Nuclear R\&D Division

- Atomic Energy International Cooperation Division

- Nuclear Safety Omice (NSO)

- Nuclear Licensing Division

- Nuclear Inspection/Enforcement Division

- Radiation Safety Division

- Nuclear Control Division

- Korea Advanced Institute of Science/Technology (KAIST)

- Korea Atomic Energy Research Institute (KAERI)

- Korea Institute of Nuclear Safety (KINS)

- Korea Institule of Geology, Mining and Materials (KIGAM)

- Korea Institute of Energy Research (KIER) 


\section{AEO}

Atomic Energy Office

Ministry of Science and Tech.

1 Chungang-dong, Kyonggi-do

Kwacheon 171-11

Tel: $\quad 82-2-503-7654$

Republic of Korea

Fax: $\quad 82-2-503-7673$

Asst.Minister, AEO

Director General, NPO

Dir., Nuclear Policy

Dir., Nuclear R\&D

Dir., Internatl. Cooperation

Director General, NSO

Dir., Nuclear Licensing

Dir., Nuc. Inspection/Enforcement

Dir., Radiation Safety

Dir., Nuclear Control

Jae Hee Hong

Hae Sool Chin

Young Wan Kwon

Tae Whan Kim

Man Ki Lee

Seung Koo Lee

Sang Sik Kang

Yung II Yu

Seong Su Yu

Dong Dae Sul

Function: License nuclear power plants and fuel cycle facilities; manage nuclear waste fund; sponsor nuclear R\&D.

\section{AEC}

Atomic Energy Commission

1, Chungang-dong

Kwacheon 171-11

Republic of Korea

Tel: $\quad 82-2-503-7646$

Fax: $\quad 82-2-507-0558$

Chairman: Deputy Prime Minister Jae Suk Chung

Function: Decision-making body for policies regarding nuclear energy; R\&D plan for nuclear fuel and nuclear energy applications; always chaired by Deputy Prime Minister; ministers of MOST and MTIE and president of KEPCO are required members. 


\section{EPB}

Electric Power Bureau

Ministry of Trade, Industry and Energy

Kwacheon 171-11

Tel: $\quad 82-2-503-9638$

Republic of Korea

Fax: $\quad 82-2-503-9649$

Dir. General, Nuclear Power

Chang Woo Rho

Function: Establish plans and policies on energy and resources, in coordination with MOST and AEO; manage nuclear fuel acquisition.

\section{KAERI}

Korea Atomic Energy Research Institute

150 Tukjin-dong

Daeduk-gu, Taejon

Tel: $\quad 82-42-868-2000$

Republic of Korea

Fax: $\quad 82-42-868-2702$

President

Dr. Jae In Shin

Sr. V.P., Nuclear

V.P., MRR Project, Dir., Nuc.

Byung Ku Kim

82-42-868-2121

Safety Research

Dir., Rad. Waste Management

Dir., Safety/Exam. Analysis

Dir., Nuclear Safety/Research

Dir., Spent Fuel Management

In Soon Chang

Hun Hwee Park

Seung Gi Ro

Chang Guy Park

Hyun Soo Park

Function: Develop reactor engineering and nuclear fuel cycle technology; assist government (MOST) with regulatory/licensing issues and in establishing national nuclear policy.

Waste Management R\&D: Fuel fabrication, uranium ore processing and conversion, radioactive waste management, and post-irradiation examination. 


\section{KAIST}

Korea Advanced Institute of

Science and Technology

373-1 Kusong-dong, Yusong-gu

Taejon 305-701, ROK

Tel: $\quad 82-42-869-2114$

Fax: $\quad 82-42-869-2210 /$

$-2220$

President

Nuclear Engineering

Dr. Soun Soon Chun

Prof. Kun Jai Lee

Function: Research-oriented graduate school, conducting advanced research and development.

\section{KEPCO}

Korea Electric Power Corporation

167 , Samsung-dong

Kangnam-Gu

Tel: $\quad 82-2-550-3114$

Seoul, Republic of Korea

Fax: $\quad 82-2-550-5981$

President

Vice President

Gen. Mgr., Nuc. Safety/Tech.

Chong Hun Lee

Dae Yong Choi

Chang Saeng Shim

Function: Develop power resources; generate/transmit electricity (operates all nuclear and conventional power plants in Korea); responsible to the government (MER).

\section{KIER}

Korea Institute of Energy and Resources

71-2 Chang-dong

Chung-gu, Taejon

Republic of Korea

President
Tel: $\quad 82-42-868-9700$

Fax: $\quad 82-42-868-9734$

Dr. Jung Moo Auh

Function: Development of energy technologies and exploitation of energy. 


\section{KINS}

Korea Institute of Nuclear

Safety Technology

P.O. Box 7

Daeduk-Danji, Taejon

Tel: $\quad 82-42-868-2601$

Republic of Korea

Fax: $\quad 82-42-868-1700$

President

Vice President

Dir., Safety Review

Director, Safety Inspection

Director, Standards Development

Yong Kyu Lim

Suk Hyung Moon

Young Soo Eun

Won Ki Shin

Sung Nyun Kim

Function: Independent regulatory organization (established 1990) to develop technical standards for nuclear safety.

\section{KIGAM}

Korean Institute of Geology,

Mining and Materials

30 Gajung-dong

Yusong-gu, Taejon

Tel: $\quad 82-42-868-3114$

Republic of Korea

Fax: $\quad 82-42-861-9720$

President

Dong Hak Kim

Function: Development and utilization of resources. 


\section{$\underline{\text { KNFC }}$}

Korea Nuciear Fuel Company, Ltd.

150 Tukjin-dong, Daeduk-gu

Taejon, Republic of Korea

Tel: $\quad 82-42-868-1000$

Fax: $\quad 82-42-868-2380$

President

Chang Suk Lee

Function: Develop domestic nuclear fuel fabrication.

Owners: KEPCO (95\%); KAERI (5\%).

Facilities

- Fuel Fabrication Plant, Daeduk site, 200 tU/a.

\section{KOPEC}

Korea Powrer Engineering Co., Inc.

87 Samsong-dong, Kangnam-gu

Seoul, Republic of Korea

Tel: $\quad 82-2-540-7701$

Fax: $\quad 82-2-540-4184$

President

Ki Oak Chang

Function: Architect-engineering services for nuclear and conventional power plants. 


\section{MOST}

Ministry of Science and Technology

1, Chungang-dong

Kwachon, Kyonggi-do

Tel: $\quad$ 82-2-503-7171

Republic of Korea

Fax: $\quad$ 82-2-503-7673

Minister

Vice Minister

Asst.Minister, AEO

Director General, NPO

Dir., Nuclear Policy

Dir., Nuclear R\&D

Dir., Internatl. Cooperation

Director General, NSO

Dir., Nuclear Licensing

Dir., Nuc. Inspection/Enforcement

Dir., Radiation Safety

Dir., Nuclear Control

Si Joong Kim

Young Sung Han

Jae Hee Hong

Hae Sool Chin

Young Wan Kwon

Tae Whan Kim

Man Ki Lee

Seung Koo Lee

Sang Sik Kang

Yung II Yu

Seong Su Yu

Dong Dae Sul

Function: Authority over virtually all scientific and technological efforts in Korea.

\section{MTIE}

Ministry of Trade, Industry

and Energy

1, Chungang-dong

Kwacheon, Kyonggi-do

Republic of Korea

Tel: $\quad 82-2-503-9641$

Fax: $\quad 82-2-503-9649$

Minister

Vice Minister

Tak Soo Kim

Director General, EPB

Dong Hoon Lee

Chang Woo Rho

Function: Lead government agency in power development and resource utilization. 


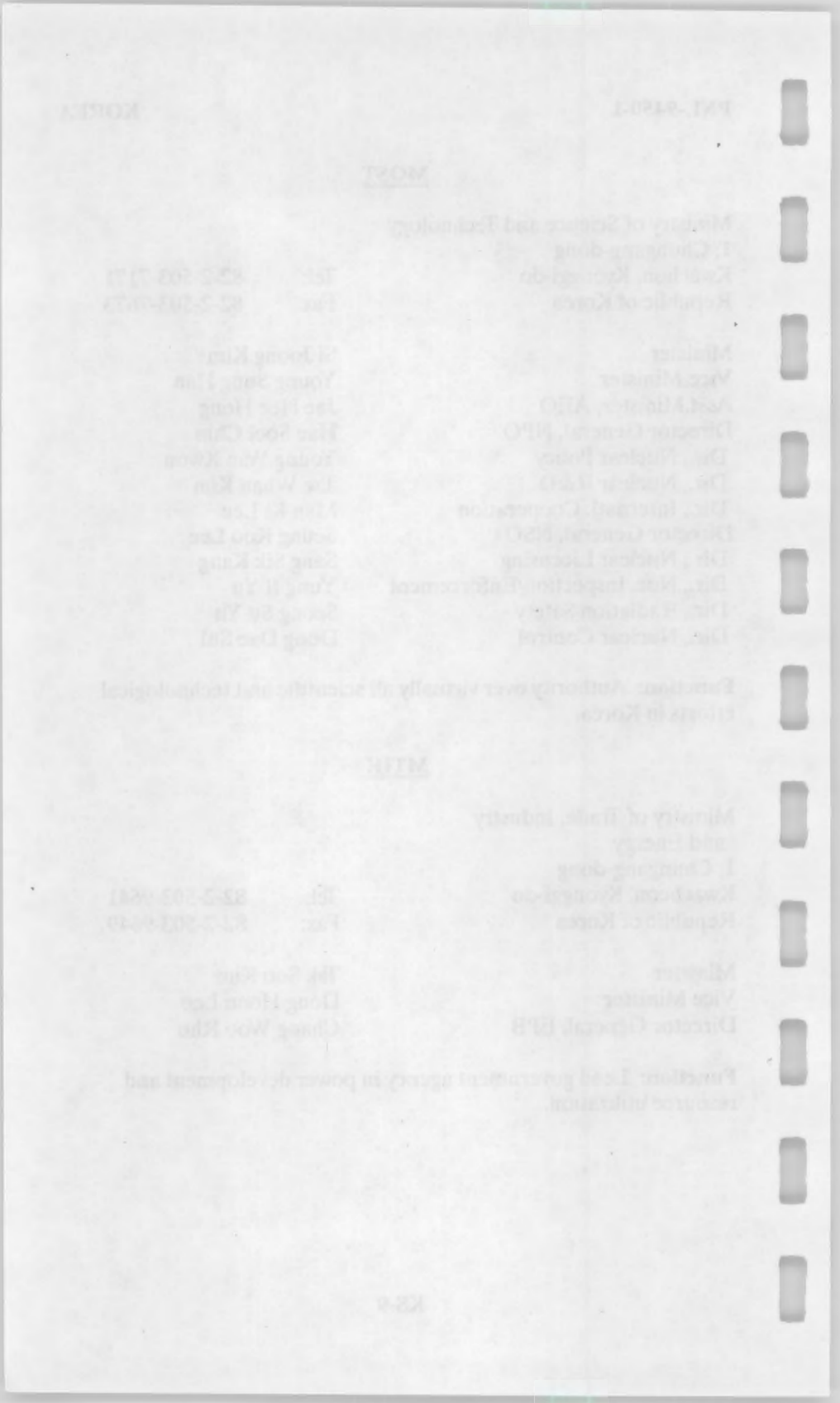




\section{NETHERLANDS}

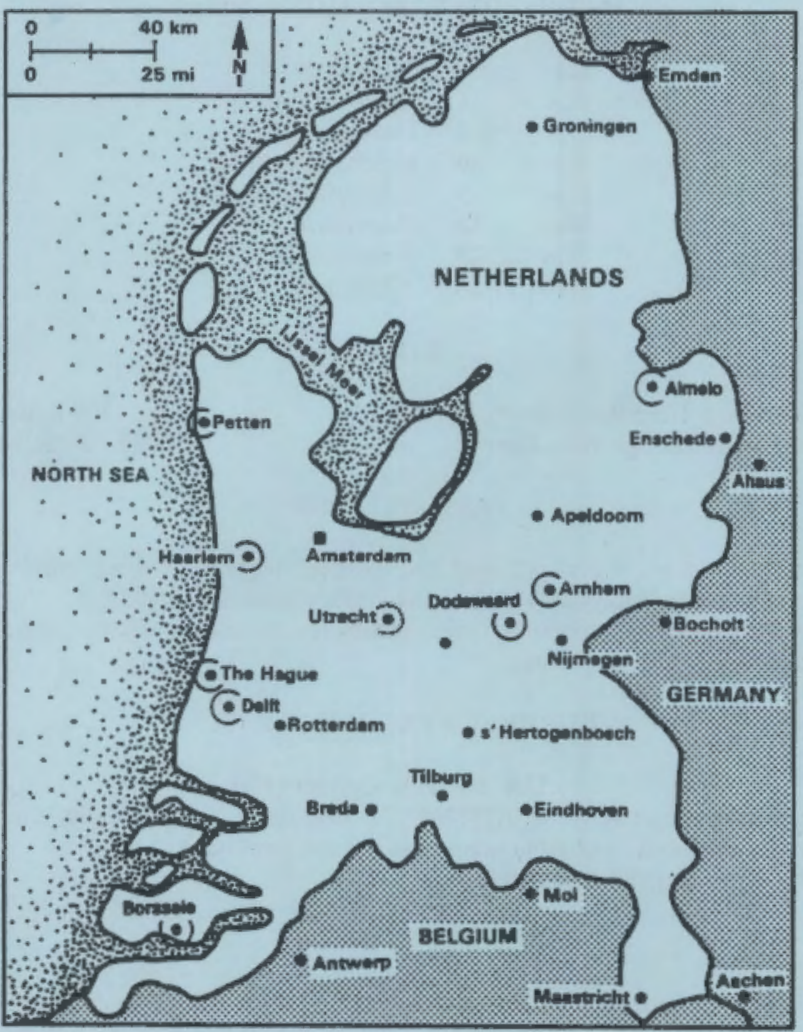


NETHERLANDS

\section{MAJOR PUBLIC HOLIDAYS (1994)}

$\begin{array}{lrl}\text { Jan } & 1 & \text { New Year } \\ \text { Apr } & 1 & \text { Good Friday } \\ \text { Apr } & 3-4 & \text { Easter } \\ \text { Apr } & 30 & \text { Qneen's Birthday } \\ \text { May } & 5 & \text { Liberation Day } \\ \text { May } & 12 & \text { Ascension } \\ \text { May } & 22-23 & \text { Pentecost } \\ \text { Dec } & 25-26 & \text { Christmas }\end{array}$

TIME

Standard Time Washington, D.C.

Daylight Savings Time Period:

\section{PASSPORT/VISA}

A passport is needed to depart and re-enter the U.S. A visa is currently not required for a visit to the Netherlands; however, it is recommended to consnit a travel agency for up-to-date information concerning requirements.

\section{CURRENCY EXCHANGE RATE}

1 U.S. $\$=1.94$ Guilder (FI.)

per Wall Street Journal, 01/31/94. Because rates fluctuate daily, it is recommended to obtain current rates from local banks or newspapers prior to departure.

\section{DIRFCT DIALING}

Individual nnmbers for direct dial to the Netherlands are complete as listed, after dialing international access code: 011. Country code is 31; listed local numbers include city code.

\section{U.S. EMBASSY - THE HAGUE}

American Embassy

Lange Voorhout 102

2514 E The Hague

Netherlands

Tel: $\quad 31-70-310-9209$

Fax: $\quad 31-70-361-4688$

Economic Section

Daniel T. Fantozzi 
PNL-9450-1

NETHERIANDS

Population

1992

15 million

ENERGY

Electric Power Capacity 1992

1995

17.4 GWe $3 \%$ nuclear

$19.8 \mathrm{GWe}$

$3 \%$ nuclear

$2000 \quad 20.1$ GWe

$3 \%$ nuclear

Electric Power Production 1992

$\begin{aligned} 77.2 & \text { TWh } \\ 56 \% & \text { gas } \\ 33 \% & \text { coal } \\ 5 \% & \text { nuclen } \\ 4 \% & \text { oil } \\ 2 \% & \text { solids } \\ 5 \% & \text { nuclear } \\ 4 \% & \text { nuclear }\end{aligned}$

NUCLEAR POWER

Polley: Expansion of nuclear capacity is on indefinite hold as a consequence of events at Chernotyl.

Nuclear Power Capacity 1992 1995

0.5 GWe

2000

$0.5 \mathrm{GWe}$

0.5 GWe

Reactor Mix

1993

BWR 1 (1969)

PWR 1 (1973)

\section{INDUSTRIAL FUEL CYCLE}

Policy: Use foreign services (fuel fabrication, reprocessing); participate with FRG and U.K. in URENCO (uranium enrichment consortium). 
Waste Management Strategy: Utilize single centralized waste collection service; extend interim storage of all wastes (50-100 years) until decisions are made regarding disposal; studies on final disposal of all radioactive wastes in geological formations are executed in the framework of the national research program (OPLA); ocean dumping of LLW and ILW has been terminated; the Netherlands contributed to NEA feasibility study regarding subseabed disposal; feasibility of disposal within international or bilateral framework is also being explored.

$\begin{array}{lll}\text { Cumulative SF Arisings (LWR) } & 1990 & 228 \mathrm{tU} \\ & 2000 & 369 \mathrm{tU}\end{array}$

\section{INTERNATIONAL RELATIONSHIPS}

Member of EC, IAEA, and OECD/NEA.

\section{ORGANIZATION}

- Government--Ministries of Economic Affairs; Housing, Physical Planning/Environment; and Social Affairs/Employment exercise overall control of nuclear matters with Parliamentary approval of their decisions.

- COVRA (Centrale Organisatie Voor Radioactief Afval)--stores and collects all radiosctive wastes.

- Interim Storage Center, 1994.

- ECN (Netherlands Energy Research Foundation)-- provides nuclear-related services, including waste treatment and disposal research.

- ILONA (Integrated National Research for Nuclear Waste - Policy Committee)--supervises and coordinates waste disposal research.

Major Milestone

- Interim Storage Center 


\section{COVRA (Central Onganization for Radioactive Waste)}

Centrale Organisatie Voor

Radioactief Afval

Spanjeweg 1, P.O. Box 34

4453 ZG s'-Heerenbroek

Netherlands

Tel: $\quad 31-1196-13900$

Fax: $\quad 31-1196-13950$

Director

Radiation Protection

Dr. Jan Vrijen

Waste Storage/Transportation

Dr. H.D.K. Codee

U. Bakema

Function: Responsible for collection, treatment, and storage of all waste (multi-funded: utilities, government, ECN).

\section{Facilities}

- Interim storage center (located in Borsele)--for all radioactive wastes; scheduled to be fully operational in 1994.

\section{ECN (Netherlands Enengy Research Center)}

Stichting Energieonderzoek

Centrum Nederland

Westerduinweg 3

Postbus 1

1755 ZG Petten, Netherlands

Tel: $\quad 31-2246-4949$

Fax: $\quad 31-2246-4480$

Chairman, Goveruing Board

Dr. G. M. V. van Aardenne

Function: Organize and sponsor energy research and development (partially government-funded).

(contd next page) 
$\underline{\text { ECN }}$ (contd)

Research Center

Managing Director

Nuclear Energy Research

Nuc. Waste/Geologic Disposal

Safety Assessment

Radionuclide Migration

Actinide Burning
Prof. Dr. H. H. van den Kroonenberg

Dr. A. M. Versteegh

L. H. Vons

Dr. J. Prij

Dr. A van Dalen

Dr. A. Abrahams

Function: Scientific and technical center, applied energy research; waste treatment.

Waste Management R\&D: Geologic waste isolation in salt dome repositories (conceptual design, thermo-mechanical, safety, and radionuclide migration studies), seabed disposal, actinide burning, and decontamination study of large components.

\section{GEOLOGICAL SURVEY OF THE NETHERLANDS}

Geological Survey of the Netherlands

Nieuwe Gracht 13

Postbus 157

Tel: $\quad 31-23-319362$

2000 AD Haarlem, Netherlands

Fax: $\quad 31-23-351614$

Director

C. Staudt

Deep Subsurface Dept.

Dr. H. M. van Montfrans 


\section{KEMA (Research/Testing Electrochemical Materlals Company)}

N.V. Tot Keuring van Elektrotechnische Materialen Arnhem Utrechtseweg 310

Postbus 9035 6800 ET Arnhem, Netherlands Tel: $\quad 31-85-569111$

Fax: $\quad 31-85-515606$

R\&D Division

Nuc. Research Program

Dr. A. H. M. Verkooijen

Quality Assurance J. B. W. Kanij

High-Level Waste Dr. H. A. W. Cornelissen Aqueous Waste Mgmt. Dr. F. J. J. G. Janssen J. L. Matteman

Function: Research and consulting development; services for utilities; waste management R\&D; characterization, quality assurance, volume reduction, and storage of radioactive wastes.

\section{MINISTRY OF ECONOMIC AFFAIRS}

Ministerie van Economische Zaken Postbus 20101 Tel: $\quad 31-70-3798911$ 2500 EC Gravenhage, Netheriands Fax: $\quad 31-70-3796358$

Dir. Electricity/Nuclear Energy H.F.G. Geyzers

Radioactive Waste J. N. A. Enst 31-70-3796471 31-70-3797849 


\section{MINISTRY OF HOUSING, PHYSICAL PLANNING AND ENVIRONMENT}

Ministerie van Volkshuisvestling

Ruimtelijke Ordening en

Milieubeheer

Postbus 450

dr. v.d. Stamstr. 2

2260 MB Leidschendam

Netherlands

Tel: $\quad 31-70-3174174$

Fax: $\quad 31-70-3175017$

Director, Rad. Protection

Dr. C. M. Plug/

R.J.P. Cornet

Radioactive Waste

Dr. A. Comelissen

\section{MINISTRY OF SOCIAL AFFAIRS AND EMPLOYMENT}

Ministry of Social Affairs and Employment

Postbus 90801

2509 LV The Hague, Netherlands

Tel: $\quad 31-70-3335549$

Fax: $\quad 31-70-3334018$

Nuclear Safety

J. Versteeg

\section{RIVM (National Institute of Public Health} and Environment Protection)

Rijksinstituut voor Volksgezondheid en Milieuhygiene

Antonie van Leeuwenhoeklaan 9

Postbus 1

Tel: $\quad 31-30-749111$

3720 BA Bilthoven, Netherlands

Safety Assessment of

Fax: $\quad 31-30-742971$

Underground Disposal Studies

Dr. P. Glasbergen

31-30-743397 


\section{PAKISTAN}

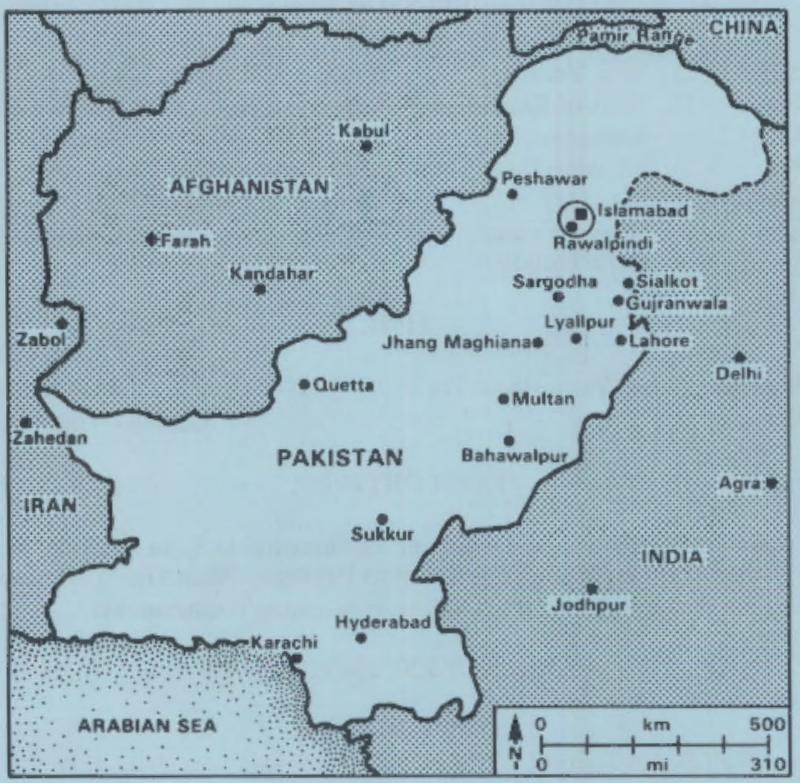


PAKISTAN

MAJOR PUBLIC HOLIDAYS (1994)

$\begin{array}{lrll}\text { Jan } & 1 & \text { New Year } \\ \text { Feb } & 12 & \text { Start of Ramadan } \\ \text { Mar } & 13-15 & \text { Ramadan } \\ \text { Mar } & 23 & \text { Pakistan Day } \\ \text { May } & 1 & \text { May Day } \\ \text { May } & 21-23 & \text { Sacrifice Feast } \\ \text { Jul } & 4 & \text { Bank Holiday }\end{array}$

Jan 1 New Year

Feb 12 Start of Ramadan

Mar 13-15 Ramadan

Mar 23 Pakistan Day

May 21-23 Sacrifice Feast

Jul

4 Bank Holiday
Aug 14 Independence

Aug 21 Prophet's Birthday

Sep 6 Defense of Pakistan

Sep 11 Death of Quaid-i-Azam

Noy 9 Iqbal Day

Dec 25 Quaid-i-Azam Birthday

Dec 31 Bank Holiday

\section{TIME}

Standard Time Washington, D.C. Work week:

\section{PASSPORT/VISA}

A passport is needed to depart and re-enter the U.S.; in addition, a visa is currently required for a visit to Pakistan. Most travel agencies can provide up-to-date information conceming requirements.

\section{CURRENCY EXCHANGE RATE}

1 U.S. $\$=30.13$ Rupee

per Wall Street Journal, 01/31/94. As rates fluctuate daily, it is recommended to obtain current rates from local banks or newspapers prior to departure.

\section{DIRECT DIALING}

Individual numbers for direct dial to Pakistan are complete as listed, after dialing international access code: 011. Country code is 92; listed local numbers include city code.

\section{U.S. EMBASSY - ISLAMABAD}

American Embassy

Diplomatic Enclave, Ramna 5

P.O. Box 1048

Tel: $\quad 92-51-82-6161$

Islamabad, Pakistan

Fax: 92-51-21-4222

Economics/Science Officer

Richard Middleton 
Population $1992 \quad 100$ million

ENERGY

Electric Power Capacity $1992 \quad 13.7$ GWe

$1.5 \%$ nuclenr

$2000 \quad 13.8$ GWe

$<1 \%$ nuclear

Electric Power Production 1992

$\begin{array}{ll}54.3 & \text { TWh } \\ 64 \% & \text { gas/oil } \\ 34 \% & \text { hydro } \\ >1 \% & \text { nuclear } \\ <1 \% & \text { coal }\end{array}$

\section{NUCLEAR POWER}

Policy: Provide up to 50\% of electrical power supply with nuclear.

$\begin{array}{lrrr}\text { Nuclear Power Capacity } & 1992 & 0.1 & \text { GWe } \\ & 2000 & 0.1 & \text { GWe } \\ \text { Reactor Mix } & 1993 & \text { HWR } & 1 \text { (1972) } \\ & & \text { HWR } & 1 \text { (1999) }\end{array}$

\section{INDUSTRIAL FUEL CYCLE}

Policy: Develop complete domestic fuel cycle - uranium mining, milling, conversion, and enrichment; fuel fabrication; reprocessing.

Cumulative SF Arisings 1993 2000

$170 \mathrm{tU}$ $440 \mathrm{tU}$

\section{INTERNATIONAL RELATIONSHIPS}

Member of IAEA; agreement with U.S. and other nations on peaceful nuclear cooperation; has not signed nonproliferation treaty. 


\section{ORGANIZATION}

- PAEC - Pakistan Atomic Energy Commission--control of nuclear matters.

- PINSTECH - Pakistan Institute of Science and Technology (Rawalpindi)--fuel cycle R\&D, including laboratory-scale reprocessing.

\section{PAEC}

Pakistan Atomic Energy Commission P.O. Box 1114

Islamabad, Pakistan

Tel: $\quad 92-51-81-9030-9$

Fax: $\quad 92-51-82-4908$

Chairman

Ishfaq Ahmad

Secretary

Muhammad Azfal

Waste Management

Raze ur-Rehman

Function: Advocate increased nuclear energy generation to overcome serious energy shortages in a country substantially lacking in natural energy resources. In an effort to accelerate Pakistan's overall economic development, the Commission also promotes the use of nuclear technologies in other areas, such as enhancing agricultural production and for medical diagnosis/therapy.

(contd next page) 


\section{$\underline{\text { PAFC }}$ (contd)}

\section{Facllities $^{(a)}$}

- Fuel Fabrication Plant at Kundian - manufacturing fuel for KANUPP since 1978; located near the Chashma site, where SGN was to build a $50-100 \mathrm{tU} / \mathrm{a}$ spent fuel reprocessing plant (project started in 1974, halted in 1977).

- A. Q. Khan Research Laboralory at Kahuta - provides nuclear training and R\&D on centrifuge enrichment.

- Centrifuge Enrlchment Plant at Kahuta - 1000 centrifuges operational at starup, in 1984, with potential of additional 2000 . 3000 units; facility not under international safeguards.

\section{PINSTECH}

Pakistan Institute of

Science \& Technology

P.O. Nilore

Islamabad, Pakistan

Tel: $\quad 92-51-84-7601-9$

Fax:

\section{Director}

I. H. Qureshi

Function: Fuel cycle R\&D activities, including analytical chemistry, nuclear materials, metallurgy, fuel development, digital electronics, control instrumentation, and computational physics; basic research facilities are open to scientists/engineers from universities as well as research organizations.

(contd next page)

(a) Based on publicly available information, organizational responsibility and specific location of some facilities cannot be identified with certainty; e.g., some reports appear to discuss the same facility, but their location is referred to variously at Kahuta, Rawalpindi or Islamabad which are relatively close to each other. 


\section{PINSTECH (contd)}

\section{Facilities $^{(a)}$}

- CNS - Center for Nuclear Studies - offers a Master's course in nuclear engineering, and fulfills training requirements in health physics, nuclear medicine, instrumentation, and basic nuclear orientation.

PARR-1 - research reactor, designed for highly-enriched (90\% uranium) fuel, commissioned in 1965, is being raised from 5 MWt to $9 \mathrm{MWt}$ and converted to low-enriched (20\%) fuel in 1990.

PARR-2 - training reactor, $27 \mathrm{~kW}$, designed and built in collaboration with the Chinese Institute of Atomic Energy (Beijing), went critical in late 1989.

Reprocessing Plant, lab scale; non-radioactive startup, 1982.

- CTC - Computer Training Center - established in collaboration with a consortium of universities.

(a) Based on publicly available information, organizational responsibility and specific location of some facilities cannot be identified with certainty; e.g., some reports appear to discuss the same facility, but their location is referred to variously at Kahuta, Rawalpindi or Islamabad which are relatively close to each other. 


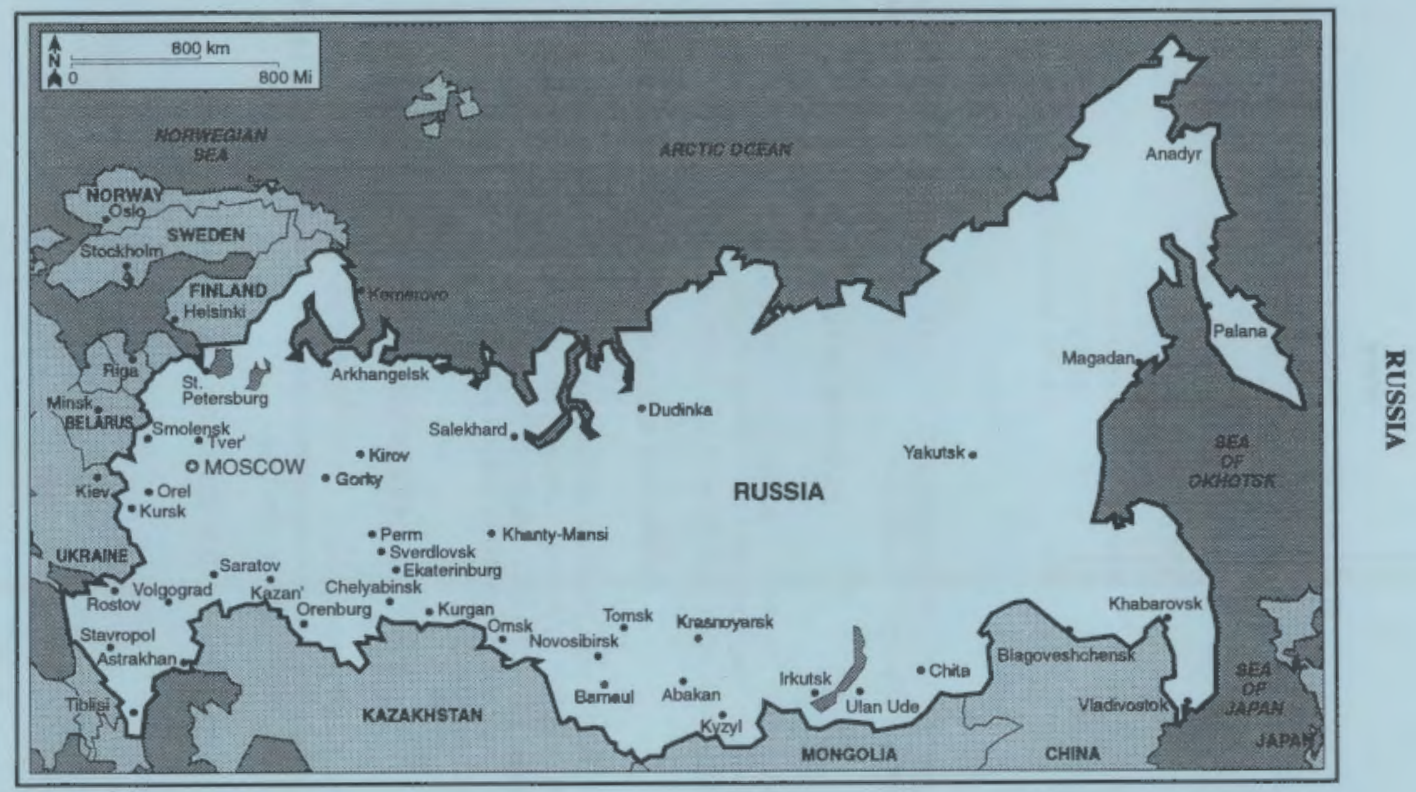




\section{RUSSIA}

\section{MAJOR PUBLIC HOLIDAYS (1994)}

$\begin{array}{lrl}\text { Jan } & 1-2 & \text { New Year } \\ \text { Jan } & 7 & \text { Christmas (Orthodox) } \\ \text { Mar } & 8 & \text { International Women's Day } \\ \text { May } & 1-2 & \text { Spring/Labor Day } \\ \text { May } & 9 & \text { Victory Day } \\ \text { June } & 12 & \text { Independence } \\ \text { Oct } & 9 & \text { Constitution } \\ \text { Now } & 7 & \text { October Revolution }\end{array}$

TIME

Standard Time Washington, D.C. Daylight Savings Time Period:

(Moscow) + 8 hours $03 / 27-09 / 24 / 94$

\section{PASSPORT/VISA}

A passport is needed to depart and re-enter the U.S.; in addition, a visa is currently required for a visit to Russia. Most travel agencies can provide up-to-date information concerning requirements.

\section{CURRENCY EXCHANGE RATE}

Local currency, the Russian Ruble, is only available upon entry into Russia. Exchange rates cannot be quoted due to continuing fluctuations. Payment in U.S. currency is apparently now prohibited. It is strongly recommended to consult with the U.S. embassy for up-todate information.

\section{DIRECT DIALING}

Individual numbers for direct dial to Russia are complete as listed, after dialing international access code: 011. Country code is 7; listed local numbers include city code.

\section{U.S. EMBASSY - MOSCOW}

American Embassy

Novinskiy Bul'var 19/23

Tel: 7-095-252-2451/

$-2459$

Moscow, Russian Federation

Fax: $7-095-956-4261$

Science Counselor

Vadimir P. Sambaiew 
Population

1992

150 million

ENERGY

Electric Power Capacity

$\begin{array}{lrl}1992 & 212 & \text { GWe } \\ & 12 \% & \text { nuclear } \\ 2000 & 245 & \text { GWe } \\ & 10 \% & \text { nuclear } \\ 2005 & 270 & \text { GWe } \\ 11 \% & \text { nuclear }\end{array}$

Electric Power Production 1992

$\begin{aligned} 1066 & \text { TWh } \\ 43 \% & \text { gas } \\ 21 \% & \text { coal } \\ 15 \% & \text { hydro/geoth. } \\ 12 \% & \text { nuclear } \\ 9 \% & \text { oil }\end{aligned}$

\section{NUCLEAR POWER}

Policy: Major program to develop nuclear power to avoid transport of fossil fuels from east of the Ural Mountains to the more densely populated western areas.

Nuclear Power Capacity

$\begin{array}{rrl}1993 & 20.2 & \text { GWe } \\ 2000 & 25.0 & \text { GWe } \\ 2005 & 36.5 & \text { GWe } \\ & & \\ 1993 & \text { LGR } & 11(1974-90) \\ & \text { PWR } & 13(1972-93) \\ & \text { FBR } & 1(1981)\end{array}$

Reactor Development advanced PWRs (VVER-500, VVER1000, VPBER-600), LGRs (MKER800), LMFBRs

\section{INDUSTRIAL FUEL CYCLE}

Policy: Complete domestic fuel cycle capability, including enrichment, fuel fabrication ( $\mathrm{UO}_{2}$ and $\mathrm{MOX}$ ), and reprocessing; complete fuel cycle services, including SF storage and LLW/ILW dispasal; shift to PWRs (since Chernobyl accident in 1986). 
Waste Management Strategy: Spent nuclear fuels from PWRs are stored 3-10 years, followed by reprocessing to recycle fissile materials and separate a number of other specific radionuclides for beneficial uses and different disposition; HLW is vitrified for disposal in a future geologic repository; HLW partitioning processes are being developed to recover most long-lived radionuclides. SF from RBMK (Soviet acronym for light-water-cooled, graphite-moderated) reactors is stored, pending decision on ultimate disposition.

Liquid LLW from nuclear reactor operations is currently evaporated, incorporated into bitumen or cement, and stored and/or disposed of at disposal facilities at each reactor station. 36 other, regional facilities exist for medical, industrial, and radioactive waste disposal. Efforts are underway to decrease liquid LLW volumes and to recycle them in water and reactant circuits.

Solid LLW, compacted at each nuclear power station, is stored/ disposed of at reactor sites; regional burial facilities are being considered to minimize transportation-related risks.

\section{INTERNATIONAL RELATIONSHIPS}

\section{DOE/MAPI}

Agreement on Scientific and Technical Cooperation in the Field of Peaceful Uses of Atomic Energy

Term: $\quad 05-25-90$ to $05-24-95$ (initiated 1973)

Scope: Technology information exchange.

\section{DOE/MAPI}

Memorandum of Cooperation in the Fields of Environmental Restoration and Waste Management

Term: $\quad 09-18-90$ to $09-17-95$

Scope: Technology information exchange related to policy and practices; evaluation of problems in environrnental remediation, D\&D of facilities and materials; R\&D; analysis/ investigations of waste partitioning; vitrification, and geologic disposal of radioactive wastes.

Member of IAEA and WANO. 


\section{GOSATOMNADZOR}

(Russian Federal Authority for Nuciear/Radiation Safety)

GOSATOMNADZOR

Taganskaya Str. 34

109147 Moscow

Russia

Chairman

Deputy Chairman

Administration
Tel: 7-095-272-3911

Fax: 7-095-278-8090

Yuri G. Vichnevsky

Yuri N. Zubkov

Valentin A. Rekunov

Function: Supervision of all safety aspects of Russian nuclear industry.

\section{KHLOPIN}

Research Production Association

V. G. Khlopin Radium Institute

2nd Murinski ave. 28

194021 St. Petersburg

Russia

Tel: 7-812-247-5737

Director-General

Deputy Directors

Laboratory Manager

Chief Scientist

Fax: 7-812-534-7752

Prof. Alexander I.

Karelin

Dr. Evgeny B. Anderson

Dr. A A Rimsky-

Korzakov

Dr. Valeriy $\mathbf{N}$.

Romanovskiy

7-812-247-6522

Dr. Leonard N. Lasarev

Activities: Development of SF treatment (reprocessing, thermal decladding, meltdown of hulls), improved HLW partitioning, waste immobilization, off-gas treatment, ${ }^{5} \mathrm{Kr}$ storage, waste disposal, geochemistry, studies on solidified waste properties; environmental remediation, protection and monitoring.

(contd next page) 


\section{KHILOPIN (contd)}

\section{Facilities}

- Ecology Laboratory (located $90 \mathrm{~km}$ from St. Petersburg in Sosnovo Bor)

Studies conducted on ecological aftermath of Chernobyl; impact of radionuclides, assessment of dose/risk; methods for monitoring radioactivity in the environment.

- Reprocessing Research \& Development Facility Mission: Develop LWR fuel reprocessing technology.

Design Basls: Chop-leach head-end; PUREX flowsheet; capacity, $3 \mathrm{~kg} / \mathrm{d}$ uranium.

History: Startup, 1973.

\section{KURCHATOV}

Russian Scientific Centre

Kurchatov Institute

Kurchatov Square 1

123182 Moscow, Russia

Tel: 7-095-196-7300

Fax: 7-095-196-2073

President

Acad. Evgeniy P.

Vice-President Velikhov

Acad. Nicolai $\mathbf{N}$.

Ponomarev-Stepnoy

Nuclear Safety

Ilya V. Elkin

Yuri P. Buzulukov

Function: Nuclear power research; R\&D on LLW/LW. 


\section{ORGANIZATION}

\section{Nuclear Program Control}

- MINATOM RF (Ministry for Atomic Energy, Russian Federation)--civilian and defense nuclear fuel cycle; waste management; successor to MAPI (Ministry of Atomic Power and Industry).

- Ministry for Ecology and Natural Resources of the Russian Federation.

- GOSATOMNADZOR (Russian Federal Authority for Nuclear and Radiation Safety).

\section{Research and Development}

- All-Russian Scientilic Research Institule for Inorganlc Malerials, Moscow-- reprocessing, solidification, and dispasal of HLW; properties of solid waste forms).

- All-Russian Scientific Research Institute for Nuclear Power Plants, Moscow-reprocessing/disposal of NPP radioactive wastes.

- Institule of Physical Chemistry, Moscow, (branch of the Russian Academy of Sciences)--radionuclide migration; waste form properties.

- Kurchatov Institule (Russian Scientific Centre), Moscow-nuclear power R\&D.

- RPA V. G. Khlopin Radium Institute, St. Petersburg--reprocessing, HLW partitioning/solidification, solid waste form properties, off-gases, waste storage/disposal, environmental remediation.

- Scientific Research Institule of Chemical Engineering, Ekaterinburg--vitrification/bitumenization pilot plants. 
- VNIPIET (All-Russian Design and Research Association for Energy Technology), St. Petersburg--design of SF reprocessing facilities, SF transportation/storage.

\section{ALL-RUSSIAN SCIENTIFIC RESEARCH INSTITUTE FOR INORGANIC MATERIALS}

All-Russian Scientific Research

Institute for Inorganic Materials

Rogov Str. 5a

Tel: $7-095-377-0104$

123060 Moscow, Russia

Fax: $7-095-376-8333$

Director

Dr. Mikhail I. Solonin

Deputy Director

Dr. Anatoliy C. Mamaev

Function: R\&D on SF reprocessing, radioactive waste processing/ solidification (bitumenization/vitrification, etc.), off-gases.

\section{ALL-RUSSIAN SCIENTIFIC RESEARCH INSTITUTE FOR NUCLEAR POWER PLANTS}

All-Russian Scientific Research

Institute for Nuclear Power Plants

Ferganskaya Str. 25

109507 Moscow, Russia

Tel: $7-095-376-1550$

Fax: 7-095-376-8333

Director General

Department Head

Prof. A. Armen Abagyan Leonid P. Khanyanov

Function: Processing and disposal of NPP radioactive wastes; decontamination of equipment//acilities; emergency situation studies. 


\section{MAYAK}

Production Association 'MAYAK'

Leninstr. 31

454065 Chelyabinsk- 65

Tel: 7-351-513-1659

Russian Federation

Fax: 7-351-513-3826

Director

Chief Engineer

Dir., Radiochemical Plant

Chief Engineer, Radiochem. Plant

Victor Fetisov

Alexander P. Suslov

Vladimir K. Sazhnov

Evgeniy G. Dzekun

Location: Near city of Kyshtym.

Function: Nuclear complex with multitude of activities and facilities, including radiochemical processing, weapon materials production reactors, isotope production, special waste storage, and burial sites; produced first Soviet weapons-grade plutonium.

\section{Facilities}

- Power Reactor Fuel Reprocessing Plant Design Basls: Started reprocessing VVER-440 reactor fuel in 1976 , with about $2000 \mathrm{tU}$ reprocessed to 1989.

\section{- Fully Radioactive HLW Vitrification Plant}

Design Basis: Single-stage joule-heated ceramic melter with a feed rate of 500-liter/h; about $160 \mathrm{t}$ of HLW phosphate glass was produced from 1987-1988; melter was shut down due to electrode problems; similar melter was modified and started operation in 6/1991. As of $4 / 1992,50 \mathrm{MCi}$ of HLW have been incorporated into phosphate glass. 


\section{MINATOM}

Ministry for Atomic Energy of the Russian Federation

Staromonetny per. 26

109180 Moscow, Russia

Minister

First Deputy Minister

Deputy Ministers

Tel: 7-095-233-1718

Fax: 7-095-230-2420

Viktor N. Mikhailov

Vitaly F. Konovalov

Nikolai N. Egorov

Yuri I. Tychkov

Alexander G. Meskov

Alexander Usanov

Evgeny Reshetnikov

Victor A Sidorenkov

Alexander G. Makarov

International Relations

Mikhail N. Ryzhov

Function: Manages all aspects of nuclear power industry.

Established 1/92 on an interim basis; successor to MAPI, the former USSR Ministry of Atomic Power and Industry.

\section{MINING/CHEMICAL ENTERPRISE}

Mining and Chemical

Enterprise

Lenin Str. 53

660033 Krasnoyarsk-33

Tel: $\quad 7-391-232-1251$

Rnssia

Fax: 7-391-232-0374

Director

Chief Engineer

Dep. Chief Engineer

Dir., Radiochemical Plant

Chief Eng., Radiochemistry

Valeriy A. Lebedev

Yuri S. Volzhanin

Yuri A. Revenko

Gennadi A. Demidov

Yuri P. Sorokin

Function: SF reprocessing, waste management, underground disposal. 


\section{MINISTRY FOR ECOLOGY/NATURAL RESOURCES}

Ministry for Ecology and

Natural Resources

B. Gruzinskaya Str. $4 / 6$

123812 GSP Moscow, Russia

Tel: $7-095-252-2305$

Fax: 7-095-254-8283

Minister

Victor I.

Deputy Minister

Danilov-Danilyan

Nikolai G. Rybalskiy

Function: Responsible for control and standardization of releases containing radionuclides.

\section{RADON}

Research Production Association RADON

7th Rostovski per. 2/14

119121 Moscow, Russia

Tel: $7-095-248-1911$

Fax: 7-095-248-1941

Director-General

Dr. Igor A. Sobolev

Vice Director-General

Dr. Sergey A. Dimitriev

Specialist

Dr. A. P. Kobelyev

Function: Research-and-production association; disposal of institutional radioactive/hazardous waste, $\mathrm{R} \& \mathrm{D}$ on waste treatment/ conditioning, engineering design/support services, environmental protection services, special accident-related emergency services/ investigations.

\section{Facilities}

- Sergiev Posad Disposal Site (formerly Zagorsk, located $75 \mathrm{~km} \mathrm{NE}$ of Moscow)

Largest facility (ca. 170 acres) with capacity for $3500 \mathrm{~m}^{2}$ (including $500 \mathrm{~m}^{3}$ liquids) waste per year (capacity at 15 other facilities $<1000 \mathrm{~m}^{2} / \mathrm{a}$ each); waste from scientific, industrial, medical, and other producers; waste characteristics similar to reactor waste, including spent radiation sources, liquid concentrates, combustible

(cont nexi page) 


\section{RADON (contd)}

liquids, highly active research reactor core components (with short-lived nuclides and limited alpha emitters); treatment/ conditioning of wastes is by compaction, combustion, cementation, bitumenization, vitrification, and special immobilization in metal matrix; disposal is in engineered concrete in-ground structures.

\section{SCIENTIFIC RESEARCH INSTITUTE OF CHEMICAL ENGINEERING}

Scientific Research Institute

of Chemical Engineering

Griboyedov Str. 32

620010 Ekaterinburg

Tel: $7-347-227-4303$

Russia

Fax: 7-343-227-5505

\section{Facilities}

- KS-KT-100 (cold pilot plant-HLW vitrification) Mission: Develop waste vitrification technology.

Design Basis: Fluid bed calciner; in-crucible melter (two-stage process); capacity, 100 liters $/ \mathrm{h}$ HLLW, $20 \mathrm{~kg} / \mathrm{h}$ glass; $160-180 \mathrm{~kg}$ glass/batch; product, phosphate glass in crucibles.

History: Startup, ca. 1975.

\section{VNIPIET \\ (All Russian Design/Research Association)}

VNIPIET

All-Russian Association

Savushkin Str. 82

197228 St. Petersburg

Russia

Tel: $\quad 7-812-239-0134$

Director-General

Fax: $7-812-239-1898$

Director-General

Chief Engineer

Dep. Chief Engineer

Prof. Vladimir A.

Kurnosov

Valentin M. Simanovsky

Dr. Vasily M. Dubrovsky

Function: Design plants/facilities for SF reprocessing, waste processing, storage/disposal; SF transport/storage; decontamination. 


\section{SOUTH AFRICA}

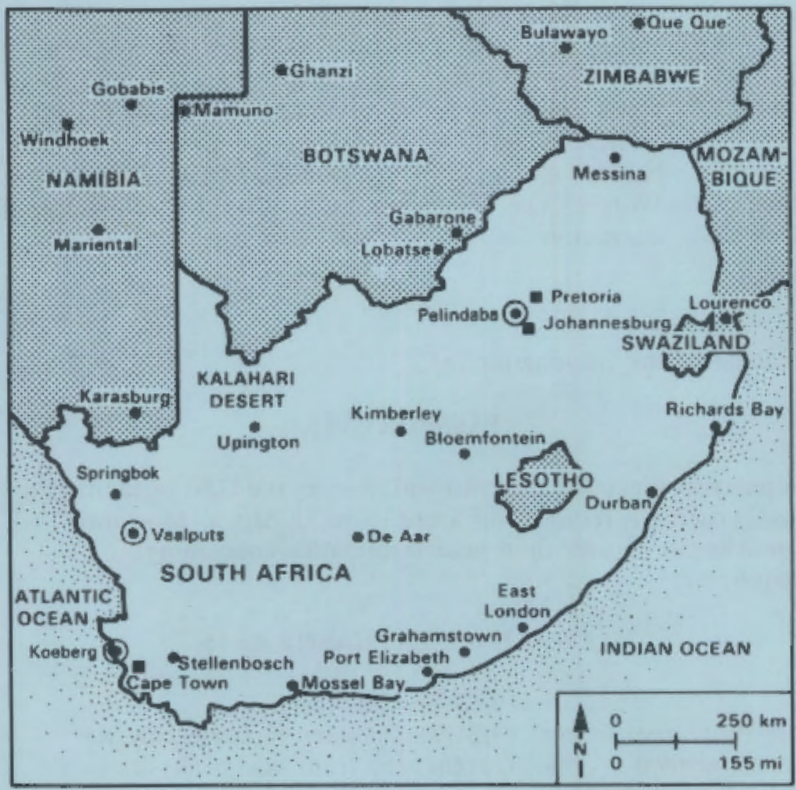


SOUTH AFRICA

MAJOR PUBLIC HOLIDAYS (1994)

$\begin{array}{lll}\text { Jan } & 1 & \text { New Year } \\ \text { Apr } & 1 & \text { Good Friday } \\ \text { Apr } & 6 & \text { Founder's Day } \\ \text { Apr } & 8 & \text { Family Day } \\ \text { May } & 1 & \text { Worker's Day } \\ \text { May } 12 & \text { Ascension }\end{array}$

May 31

Oct 10

Dec 16

Dec 25

Republic Day

Kruger Day

Dec 26

Day of the Vow

Christmas

Day of Goodwill

\section{TIME}

Standard Time Washington, D.C.

+7 hours

\section{PASSPORT/VISA}

A passport is needed to depart and re-enter the U.S.; in addition, a visa is currently required for a visit to South Africa. Most travel agencies can provide up-to-date information concerning requirements.

\section{CURRENCY EXCHANGE RATE}

1 U.S. $\$=3.42$ Rand

per Wall Street Journal, 01/31/94. As rates fluctuate daily, it is recommended to obtain current rates from local banks or newspapers prior to departure.

\section{DIRECT DIALING}

Individual numbers for direct dial to South Africa are complete as listed, after dialing international access code: 011. Country code is 27; listed local numbers include city code.

\section{U.S. EMBASSY - PRETORIA}

American Embassy

877 Pretorius St.

P.O. Box 9536

Tel: $\quad 27-12-342-1048$

Pretoria, South Africa

Fax: $\quad 27-12-342-2244$

Economic Affairs Counselor

J. Michael Cleverley 
Population

1993

39 million

\section{ENERGY}

Electric Power Capacity

1993

33.2 GWe

1995

6\% nuclear

1

1995
2000

34.1 GWe

$5 \%$ nuclear

37.9 GWe

$5 \%$ nuclear

Electric Power Production 1993

$\begin{aligned} 154 & \text { TWh } \\ 94 \% & \text { coal } \\ <5 \% & \text { nuclear } \\ 1 \% & \text { other } \\ <1 \% & \text { hydro } \\ 6 \% & \text { nuclear } \\ 6 \% & \text { nuclear }\end{aligned}$

\section{NUCLEAR POWER}

Policy: Expand electric power production capacity chiefly through coal-burning plants, but develop modest nuclear capability to complement coal, particularly post-2000.

$\begin{array}{llrl}\text { Nuclear Power Capacity } & 1993 & 1.8 & \text { GWe } \\ & 2000 & 1.8 & \text { GWe } \\ \text { Reactor Mix } & 1993 & \text { PWR } & 2(1984 / 85)\end{array}$

\section{INDUSTRIAL FUEL CYCLE}

Waste Management Stralegy: Interim storage of reactor LLW/ILW at the reactor, followed by disposal at two shallow-land disposal facilities; interim storage of spent fuel for $\sim 40$ years; plans for disposal not defined.

$\begin{array}{llll}\text { Cumulative SF Arisings (LWR) } & 1990 & 180 \mathrm{tU} \\ & 2000 & 520 \mathrm{tU}\end{array}$ 
Major Milestones

- Dry SF storage facility (Vaalputs)

INTERNATIONAL RELATIONSHIPS

Member of LAEA.

ORGANIZATION

Ministry of Mineral and Einergy A Tairs

Atomic Energy Corporation (AFC)

- Pelindaba Site

- R\&D

- Research Reactor

- Isotope Production

- Fuel Fabrication

- LLW Disposal

Vaalputs National LLW Disposal Facility

- LLW/ILW Disposal

- Site Characterization

- Valindaba Site

- Uranium Enrichment

- Uranium Conversion

National Energy Council (NEC)

Council for Nuclear Safety (CNS)

- Independent Regulatory Licensing Agency

Eskom

- Electricity Production 
Atomic Energy Corporation

of South Africa Ltd.

P.O. Box 582

Tel: $\quad 27-12-316-4911$

Pretoria 0001, South Africa

Fax: $\quad 27-12-316-3111$

Chief Executive Officer

Senior General Managers:

Nucl. Fuel Production

Dr. Waldo E. Stumpf

Technology Development

Engineering

Business Development

Sr. Mgr., Nuc. Waste Tech.

P. J. Venter

K. F. Fouche

L. S. Snyders

Dr. A. G. M. Jackson

H. J. Van der Westhuizen

Function: Overall responsibility for government nuclear activities including uranium conversion and enrichment, $R \& D$, radioisotope production, radwaste disposal and repository; fuel fabrication.

\section{Facilities}

\section{- Pelindaba Site}

Mission: Perform nuclear R\&D; operate research reactor, isotope prodnction line, food irradiation facility; manufacture fuel; operate LLW treatment/shallow-land disposal facilities.

- Vaalpuis National LLW

Disposal Facility

Private $\mathrm{Bag}$ X7

Springbok 8240, South Africa

Tel: $\quad 27-251-22832$

Fax: $\quad 27-251-81220$

Mission: Operate LLW/ILW shallow-land disposal facilities; perform site characterization and environmental studies.

Design Basis: $1,470 \mathrm{~ms} / \mathrm{a}$ LLW/LW disposal.

(contd next page) 
AEC (contd)

- Valindaba Uranium Enrichment and Conversion Plants Mission: Perform enrichment R\&D; operate semi-commercial conversion and enrichment plants. Pilot-scale operations shut down in 1990.

Design Basis: 300,000 SWU/a enrichment - $700 \mathrm{tU} / \mathrm{a}$ conversion.

\section{$\underline{\text { CNS }}$}

Council for Nuclear Safety

P.O.B. 7106

Tel: $\quad 27-12-663-5500$

Hennopsmeer 0046, South Africa

Fax: $\quad 27-12-663-5513$

Chairman

Prof. J. B. Martin

Vice-Chairman

Dr. D. Reitmann

Exec. Officer

B. C. Winkler

General Manager

J. Leaver

Function: Independent regulatory/licensing agency for construction and operation of nnclear installations (established by the 1988 Nuclear Energy Amendment Act).

\section{ESKOM}

\section{ESKOM}

P.O. Box 1091

Johannesburg 2000

South Africa

Tel: $\quad 27-11-800-8111$

Fax: $\quad 27-11-800-4390$

Chief Executive/COB

Chairman, Electricity Conncil

Exec. Dir., Technology

A. J. Morgan

Dr. John B. Maree

Alex Ham

Function: Provide electricity for public use. 


\section{SPAIN}

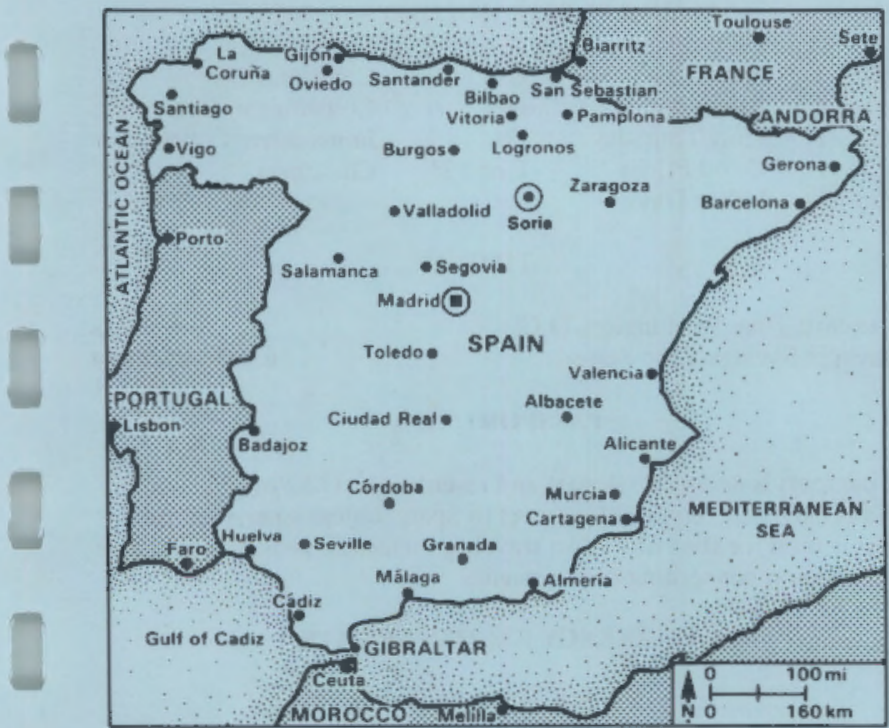


SPAIN

MAJOR PUBLIC HOLIDAYS (1994)

$\begin{array}{lrllrl}\text { Jan } & 1 & \text { New Year } & \text { Oct } & 12 & \text { Columbus Day } \\ \text { Jan } & 6 & \text { Epiphany } & \text { Dec } & 6 & \text { Constitution Day } \\ \text { Mar } 31 & \text { Holy Thursday } & \text { Dec } & 8 & \text { Immaculate Conception } \\ \text { Apr } 1 & \text { Good Friday } & \text { Dec } & 25 & \text { Christmas } \\ \text { May } & 1 & \text { Labor Day } & & & \end{array}$

TIME

Standard Time Washington, D.C.

Daylight Savings Time Period:

\section{PASSPORT/VISA}

A passport is needed to depart and re-enter the U.S.; in addition, a visa is currently required for travel to Spain, unless a personal passport is used for the visit. Most travel agencies can provide up-to-date information concerning requirements.

\section{CURRENCY EXCHANGE RATE}

1 U.S. $\$=139.86$ Peseta

per Wall Street Journal, 01/31/94. Because rates fluctuate daily, it is recommended to obtain current rates from local banks or newspapers prior to departure.

\section{DIRECT DIALING}

Individual numbers for direct dialing to Spain are complete as listed, after dialing international access code: 011 . Country code is 34; listed local numbers include city code.

\section{U.S. EMBASSY - MADRID}

American Embassy

Serrano 75

28006 Madrid, Spain

Science Attaché
Tel: $\quad 34-1-577-4000$

Fax: $\quad 34-1-577-5735$

Helen B. Lane 
PNL-9450-1

Population

1992

39 million

ENERGY

Electric Power Capacity

$\begin{array}{lll}1992 & 44.1 & \text { GWe } \\ & 16 \% & \text { nuclear } \\ 1995 & 45.7 & \text { GWe } \\ & 15 \% & \text { nuclear } \\ 2000 & 51.1 & \text { GWe } \\ & 14 \% & \text { nuclear }\end{array}$

Electric Power Production

$\begin{array}{rrl}1992 & 156.5 & \text { TWh } \\ & 42 \% & \text { coal } \\ & 36 \% & \text { nuclear } \\ & 12 \% & \text { hydro } \\ & 9 \% & \text { oil } \\ & 1 \% & \text { other } \\ 1995 & 27 \% & \text { nuclear } \\ 2000 & 23 \% & \text { nuclear }\end{array}$

\section{NUCLEAR POWER}

Pollcy: Continue to operate existing nuclear power plants.

Moratorium on new nuclear power plant construction has been in place for several years (confirmed 1991).

Nuclear Power Capacity 1992

$7.0 \mathrm{GWe}$

2000

7.0 GWe

Reactor Mix

1993

PWR 7 (1969-88)

BWR 2 (1971-85) 


\section{INDUSTRIAL FUEL CYCLE}

Pollcy: Once-through fuel cycle for LWRs; no domestic reprocessing and no further contracts for foreign reprocessing, except GCR fuel (Vandellos I).

Waste Management Stralegy: Store spent fuels at the reactor sites for at least 10 years; reracking in some reactor pools and dry storage in dual-purpose casks planned to provide additional capacity until geologic repository is ready to receive HLW (SF); granite, salt, and clay are being considered as host rock for repository; shallow-land burial of LLW in fully engineered facility at El Cabril, province of Córdoba (in operation since 10/92).

Cumulative SF Arisings (LWR)

\section{Industrial-Scale Activities (Capacity)}

- U mining/milling: $270 \mathrm{tU} / \mathrm{a}$

- U enrichment: $11.1 \%$ interest in Eurodif

- Fuel fabrication: $200 \mathrm{tU} / \mathrm{a}$

- Intermediate SF storage: $3,000 \mathrm{tU}$

\section{INTERNATIONAL RELATIONSHIPS}

DOE/Empresa Agreement in the Field of Radioactive Waste Management

Term: $12 / 16 / 92-12 / 16 / 97$

Scope: Exchange of technology for management of radioactive wastes, i.e., characterization of geologic formations; preparation/packaging; disposal; surface/subsurface storage; performance and transportation assessments; mutually agreed topics associated with management of radioactive waste.

Member of EC, IAEA, and OECD/NEA. 


\section{CIEMAT (Energy Research Center)}

Centro de Investigaciones

Energéticas, Medio Ambientales

y Tecnologicas

Avenida Complutense 22

Ciudad Universitaria

28040 Madrid, Spain

Tel: $\quad 34-1-346-6000 / 01$

Fax: 34-1-346-6005

President

General Director

Dir., Nuc. Technology

Dir., Rad. Protection

Waste Management

Luis María Atienza Serna

José Angel Azuara Solís

Manuel Montes Ponce de León

Francisco Mingot Buades

Armando Uriarte

Function: Organized into four research institutes: nuclear technology (R\&D on nuclear fuel cycle, decommissioning, material sciences, and safety analyses); fundamental research; radiological protection and environment; renewable energies.

\section{Facilities}

- Juan Vigon National Nuclear Energy Center, Madrid

\section{CSN (Council of Nuclear Safety)}

Consejo de Seguridad Nuclear

Juslo Dorado, 11

E-28020 Madrid, Spain

Tel: $\quad 34-1-346-0100$

Fax: $\quad 34-1-346-0471$

President

Commissioners

Donato Fuejo Lago

Enrique Echávarri Lozano

Fabio Sarmiento Almeida

Rafael Caro Manso

Eduardo González Gómez

Function: Independent body, responsible to Parliament, with regulatory powers on nuclear safety and radiation protection matters. 


\section{ENRESA (National Waste Management Company)}

Empresa Nacional de Residuos

Radiactivos S.A.

Emilio Vargas, 7

E-28043 Madrid, Spain

President

Tel: $\quad 34-1-519-5255$

Fax: $\quad 34-1-519-5268$

Director General

Director, Engineering International Relations

Juan M. Kindelán

34-1-279-2667

Alberto Lopez Garcia

34-1-279-2858

Aurelio M. Ulibarri

Valentín González

34-1-519-5314

Function: Provide waste management services and disposal facilities to all Spanish nuclear companies and radwaste producers; responsible to the Ministry of Industry and Energy; funded by CIEMAT $(80 \%)$ and the National Institute of Industry (20\%).

\section{Facilities}

- LLW Surface Storage Facility, El Cabril, Córdoba

\section{ENUSA (National Fuel Cycle Company)}

Empresa Nacional del Uranio S.A.

Santiago Rusinol 12

E-28040 Madrid, Spain

Tel: $\quad 34-1-347-4200$

Fax: $\quad 34-1-347-4215$

President

Alfredo Llorenze Legaz

Function: Supply fuel cycle services except waste management and disposal (uranium mining and milling, fuel fabrication) to Spanish nuclear power plants.

\section{Facilities}

- LWR Fuel Fabrication Plant

Commissioned late 1985.

Capacity: $200 \mathrm{tU} / \mathrm{a}$, can be expanded to $500 \mathrm{tU} / \mathrm{a}$. 
MINISTRY OF INDUSTRY AND ENERGY

Minister

Secretary General, Energy/Mineral Resources Director General, Energy
José Claudio

Aranzadi Martinez

Luis Maria Atienza Serna María Luisa Huidobro y Arreba 


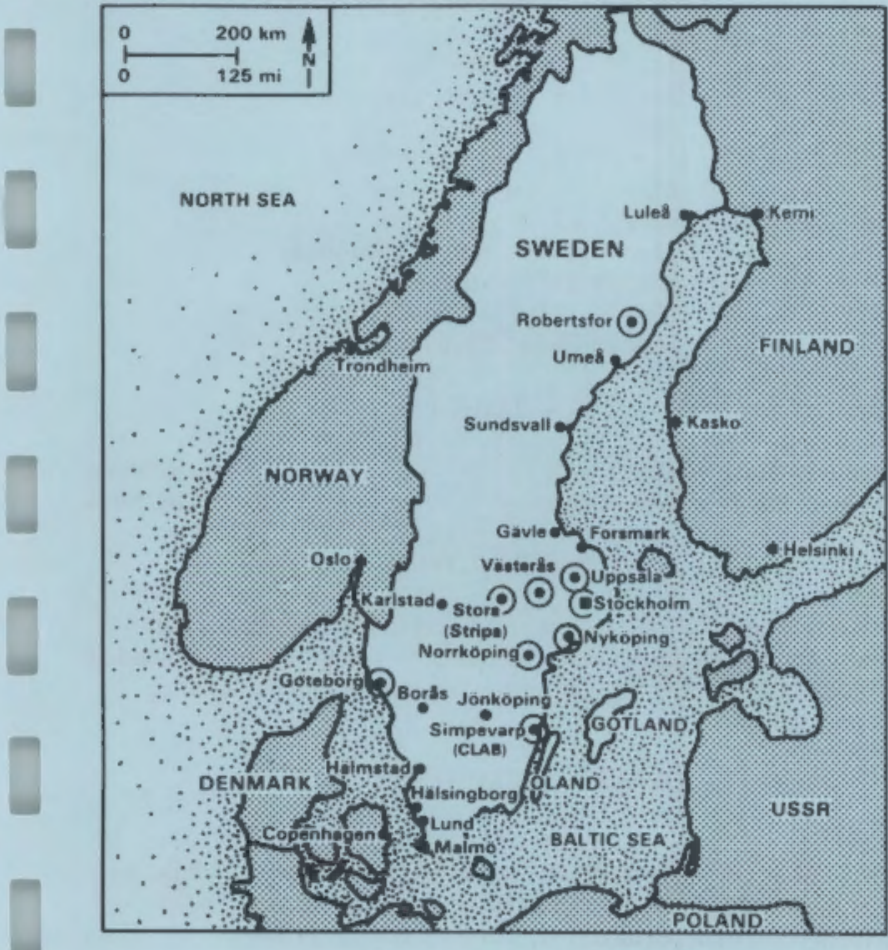




\section{SWEDEN}

\section{MAJOR PUBLIC HOLIDAYS (1994)}

$\begin{array}{lrl}\text { Jan } & 1 & \text { New Year } \\ \text { Jan } & 6 & \text { Epiphany } \\ \text { Apr } & 1 & \text { Good Friday } \\ \text { Apr } & 4 & \text { Easter Monday } \\ \text { May } & 1 & \text { Labor Day } \\ \text { May } & 12 & \text { Ascension } \\ \text { May } & 23 & \text { Pentecost Monday } \\ \text { Jun } & 24-25 & \text { Midsummer } \\ \text { Nov } & 1 & \text { All Saints } \\ \text { Dec } & 24-26 & \text { Christmas }\end{array}$

TIME

Standard Time Washington, D.C. +6 hours Daylight Savings Time Period:

\section{PASSPORT/VISA}

A passport is needed to depart and re-enter the U.S. A visa is currently not required for a visit to Sweden; however, it is recommended to consult a travel agency for up-to-date information concerning requirements.

\section{CURRENCY EXCHANGE RATE}

1 U.S. $\$=7.86$ Krona (SEK)

per Wall Street Journal, 01/31/94. Because rates fluctuate daily, it is recommended to obtain current rates from local banks or newspapers prior to departure.

\section{DIRECT DIALING}

Individual numbers for direct dial to Sweden are complete as listed, after dialing international access code: 011. Country code is 46; listed local numbers include city code.

\section{U.S. EMBASSY - STOCKHOLM}

American Embassy

Strandvagen 101 10000 Stockholm, Sweden

Economic Section
Tel: $\quad 46-8-783-5300$

Fax: $\quad 46-8-661-1964$

Kenneth H. Kolb 
$\begin{array}{llll}\text { Population } & 1992 & 8.7 \text { million }\end{array}$

\section{ENERGY}

Electric Power Capacity 1992

$\begin{array}{lll} & 30 \% & \text { nuclear } \\ 1995 & 34.8 & \text { GWe } \\ & 29 \% & \text { nuclear } \\ 2000 & 35.2 & \text { GWe } \\ & 29 \% & \text { nuclear }\end{array}$

Electric Power Production 1992

$$
\begin{array}{rrl}
1992 & 145.7 & \text { TWh } \\
& 51 \% & \text { hydro } \\
& 44 \% & \text { nuclear } \\
& 2 \% & \text { coal } \\
& 2 \% & \text { oil } \\
& 1 \% & \text { solids } \\
& 46 \% & \text { nuclear }
\end{array}
$$$$
2000 \quad 48 \% \text { nuclear }
$$

\section{NUCLEAR POWER}

Policy: Phase out all nuclear plants by the year 2010 at the latest; changing this policy will require a new decision by Parliament.

Nuclear Power Capacity 1992

2000

Reactor Mix

1993
9.9 GWe

$9.9 \mathrm{GWe}$

BWR 9 (1972-85)

PWR 3 (1975-83) 


\section{INDUSTRIAL FUEL CYCLE}

Policy: Direct disposal of spent fuel; no Pu recycle is planned; costs for waste management and future decommissioning of nuclear power plants are paid by fees collected from the nuclear utilities.

Wasle Management Stralegy: Store spent fuel for $30-40$ years in an underground pool storage facility; encapsulate spent fuel in a highly corrosion-resistant canister, emplace in a deep geologic (crystalline rock) repository.

Cumulative SF Arisings (LWR) 1993

Cumulative Waste Arisings

(conditioned and encapsulated . ready for disposal)
2020

SF

TRU 5,600 canisters Reactor waste " core comp. D\&D
$6,000 \mathrm{~m}^{3}$ $95,000 \mathrm{~m}^{3}$

$19,000 \mathrm{~m}^{3}$ $113,000 \mathrm{~m}^{3}$

\section{Industrial-Scale Activities}

- LWR fuel fabrication: $400 \mathrm{tU} / \mathrm{a}$.

Major Milestones (SF Repository)

- Star characterization of three candidate sites

- Start-up underground Hard Rock Laboratory

- Perform detailed investigations of two sites

- Submit license application

- Start repository construction

- Start repository operation 


\section{INTERNATIONAL RELATIONSHIPS}

\section{DOE/SKB Agreement for Cooperation in Waste Management}

Term: $\quad$ 07-01-77 to 09-09-95

Scope: Preparation and packaging of waste forms; storage, field and laboratory testing; geologic disposal; safety and environment, institutional and public relations issues. Collaboration in Stripa Mine test program (NEA coordination), U.S. participation in performance assessment computer model and code intercomparison sponsored by SKB.

Member of IAEA and OECD/NEA; waste management cooperative agreements with Canada, EC, Finland, France, Spain, Switzerland. Host country for NEA Stripa Project.

\section{ORGANIZATION}

- Waste Management

- SKB (Swedish Nuclear Fuel and Waste Management Company)--executes spent fuel and waste management program for the utilities; manages waste disposal R\&D programs.

\section{- Licensing Responsibilities}

- SKI (Swedish Nuclear Power Inspectorate)--licensing for construction/operations of nuclear facilities; administers waste management fund collected from nuclear utilities; oversees back-end fuel cycle activities.

- SSI (Swedish National Institute of Radiation Protection).

- National Swedish Franchise Board for Environment Protection.

- Municipality where the facility is to be located (right of veto). 


\section{CHALMERS (TECHNICAL UNIVERSITY)}

Chalmers Tekniska Hoegskola

41296 Goeteborg, Sweden

Nuclear Chemistry

Waste Management R\&D: Radionuclide transport by groundwater, sorption on natural clays and rock minerals.

\section{KEMAKTA}

Kemakta Konsult AB

Pipersgatan 27

11228 Stockholm, Sweden

Manager

Tel: $\quad 46-31-72-10-00$

Fax: $\quad 46-31-16-84-94$

Jan-Olof Liljenzin

Function: Computer calculations on hydrology/nuclide migration.

\section{KTH (Royal Institute of Technology)}

\section{KTH}

10044 Stockholm, Sweden

Chemical Engineering

Inorganic Chemistry
Tel: $\quad 46-8-790-60-00$

Fax: $\quad 46-8-109-199$

Ivars Neretnieks

I. Grenthe

Wasle Management R\&D: Near-and far-field migration modeling, rock-matrix diffusion experiments; actinide-chemistry, solubility calculations, groundwater sampling and characterization. 


\section{NUCLEAR SAFETY AND TRAINING CENTER}

Kärnkraftssäkerhet och

Utbildning AB

Bax 5864

Tel: $\quad 46-8-665-2800$

10248 Stockholm, Sweden

Fax: $\quad 46-8-782-9528$

Director

Svante Nyman

Punction: Promote coordination/cooperation among the Swedish utilities in their nuclear power plant safety work; nuclear simulator training in Sweden.

\section{SGAB (Swedish Geological Company)}

Sveriges Geologiska AB

Vretgränd 18

Box 670

75128 Uppsala, Sweden

Hydrogeology
Tel: $\quad 46-18-15-64-20$

Fax: $\quad 46-18-14-02-10$

Leif Carlsson

Wasle Management R\&D: Evaluation of rock formations for use as waste disposal sites (permeability, groundwater behavior, age, and chemistry). 


\section{$\underline{\text { SKB (Nuclear Fuel and Waste Management Company) }}$}

Svensk Kämbränslehantering $\mathrm{AB}$

Box 5864

Tel: $\quad 46-8-665-28-00$

10248 Stockholm, Sweden

Fax: $\quad 46-8-661-57-19$

President

Sten Bjurström

Vice President

$46-8-665-2803$

Per-Eric Ahlström

Systems/Facilities, Director

Director, R\&D/Safety Anal.

Geoscience 46-8-665-2834

Hans Forsström

Tönis Papp 46-8-665-2838

Lars Olof Ericsson

Fred Karlsson 46-8-665-2801

Chemistry 46-8-665-2830

Design \& Engineered Barriers

Material Sciences

Christer Svemar

Lars Werme $46-8-665-2811$

Torsten Eng 46-8-665-2825

International Relations

Nuc. WM Int'I Consult. Services

Bo Gustafsson

$$
\text { 46-8-665-2833 }
$$

46-8-665-2816

Function: Coordinate and arrange for nuclear fuel supply and reprocessing services for all Swedish nuclear power reactors; manage and fund R\&D for the back end of the fuel cycle; responsible for design, construction, and operation of all necessary storage and disposal facilities; demonstrate that SF and other long-lived wastes can be disposed of safely and permanently; provide transportation of SF outside reactor sites.

Owners: Utilities. 


\section{SKB (contd)}

\section{Facilities}

- CLAB (Central Storage for Spent Fuel, located at Simpevarp adjacent to Oskarshamn Power Station)

Mission: AFR storage facility.

Design Capacity: Initially, $3000 \mathrm{t}$; being upgraded to $5000 \mathrm{t}$.

History: Startup construction, 05/80; startup operation, 1985.

- SFR (Swedish Final Repository for LLW and ILW, located in rock $50 \mathrm{~m}$ below seabed, $1 \mathrm{~km}$ outside Forsmark harbor on Gulf of Bothnia).

Design: Concrete silos inside cylindrical rock caverns isolated by layer of bentonite clay backfill between silo and rock for highactivity ILW; conventional tunnel rooms for LLW; low-activity ILW is in concrete-walled "hot-cells" in tunnels; $1-\mathrm{km}$-long tunnels leading to repository to be plugged with concrete.

Capacity: $90,000 \mathrm{~m}^{3}$.

History: Phase-1 construction startup, 1983; operation startup, 1988; Phase-2 operations startup, late 1990s.

\section{- Äspö Hard Rock Leboratory}

SKB Development/Äspö Hard Rock Laboratory

Project Office

Box 5864

10248 Stockholm, Sweden

Tel: $\quad 46-8-665-2831$

Fax: $\quad 46-8-665-5719$

Project Manager

Göran Bäckblom

SKB Äspö Hard Rock Laboratory

Site Office, Pl 300

57093 Figeholm, Sweden

Tel: $\quad 46-491-82000$

Fax: $\quad 46-491-82005$

Site Manager

Olle Zellman

Underground research laboratory (located on Äspö Island at Simpevarp) under construction; starup/operation 1994. 


\section{SKI (Nuclear Power Inspectorate)}

Statens Kärnkraftinspektion

Box 27106

Tel: $\quad 46-8-665-44-00$

10252 Stockholm, Sweden

Fax: $\quad 46-8-661-90-86$

Director

Lars-Olof Högberg

Waste Management

Soeren Norrby

Function: Responsible for licensing nuclear facilities; administers waste management fund collected from nuclear utilities; oversees back-end fuel cycle activities.

\section{SSI (National Institute of Radiation Protection)}

Statens Straalskyddsinstitut

Box 60204

Tel: $\quad 46-8-729-71-00$

10401 Stockholm, Sweden

Fax: $\quad 46-8-729-71-08$

Director

Radwaste Group, Head (A)

Gunnar Bengtsson

Gunnar Johansson

Function: Responsible for establishing and enforcing radiation protection regulations.

\section{STUDSVIK RADWASTE AB}

Studsvik Radwaste AB

61182 Nyköping, Sweden

Director
Tel: $\quad 46-155-210-00$

Fax: $\quad 46-155-630-44$

Olle Andersson

Function: Nuclear waste R\&D and services to support Swedish power programs (contract research) on LLW and ILW treatment, $\mathrm{D} \& \mathrm{D}$ techniques, $\mathrm{SF}$ leaching, biosphere migration, dose calculations.

Owner: Vattenfall (via Studsvik AB). 


\section{VATTENFALL}

Vattensfall

16287 Vaellingby

Sweden

Tel: $\quad 46-8-739-50-00$

Fax: $\quad 46-8-737-01-70$

President

Nuclear Power

Carl-Eric Nyquist

Stig Sandklef

Function: Operate the power distribution grid in Sweden; produce power (owner of Ringhals Nuclear Power Plants). 


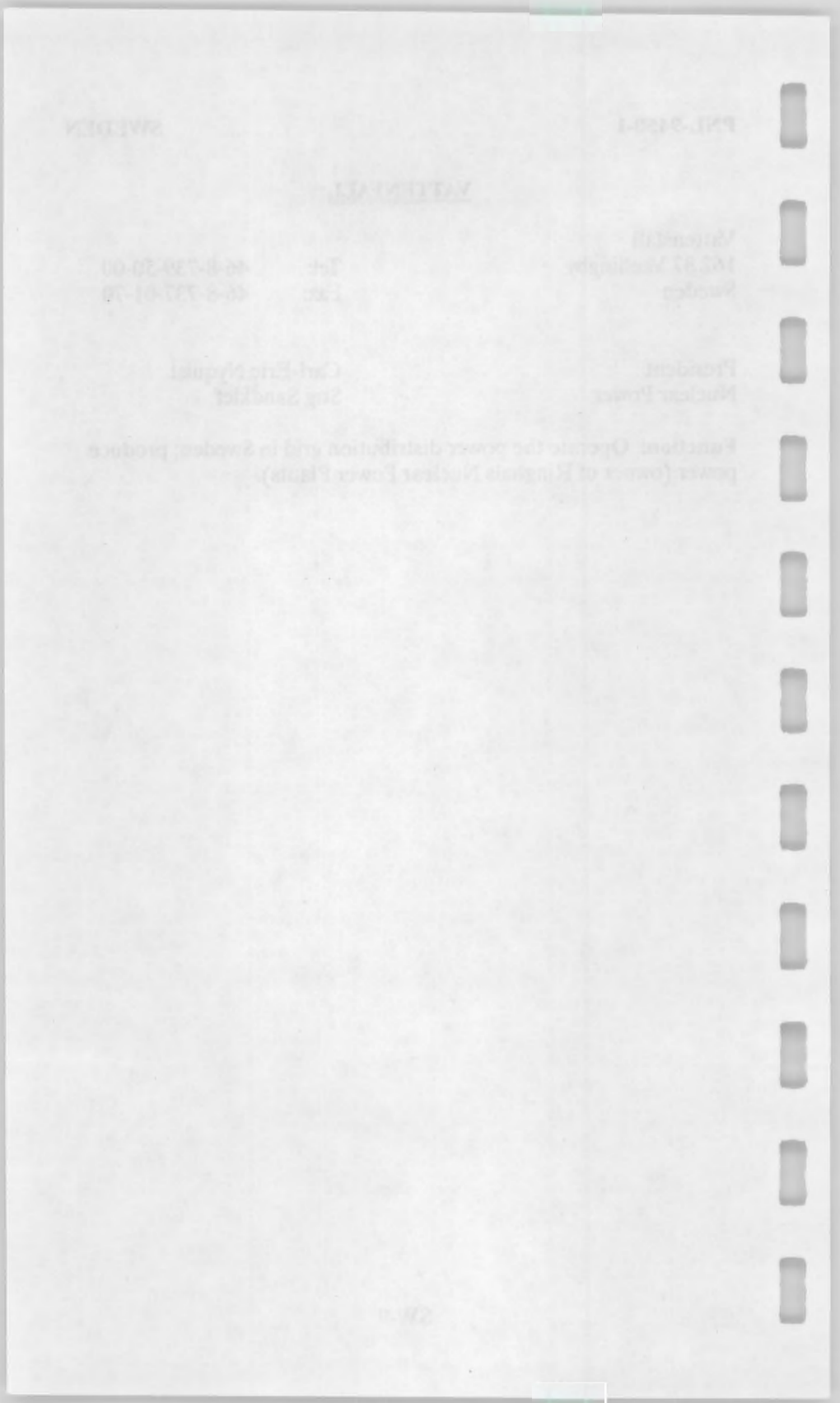




\section{SWITZERLAND}

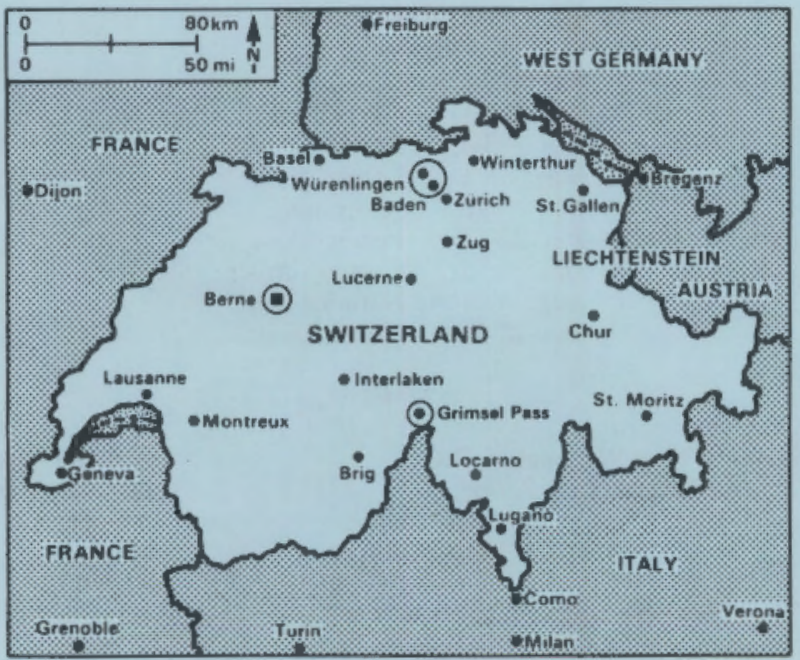




\section{SWITZERLAND}

\section{MAJOR PUBLIC HOLIDAYS (1994)}

$\begin{array}{lrl}\text { Jan } & 1 & \text { New Year } \\ \text { Apr } & 1 & \text { Good Friday } \\ \text { Apr } & 3-4 & \text { Easter } \\ \text { May } & 1 & \text { Labor Day } \\ \text { May } & 12 & \text { Ascension } \\ \text { Jun } & 22-23 & \text { Pentecost } \\ \text { Jun } & 5 & \text { Corpus Christi } \\ \text { Aug } & 1 & \text { National Day } \\ \text { Dec } 25-26 & \text { Christmas }\end{array}$

TIME

Standard Time Washington, D.C.

Daylight Savings Time Period:

\section{PASSPORT/VISA}

A passport is needed to depart and re-enter the U.S.; a visa is currently not required for a visit to Switzerland; however, it is recommended to consult a travel agency for up-to-date information concerning requirements.

\section{CURRENCY EXCHANGE RATE}

1 U.S. $\$=1.45$ Franc

per Wall Street Journal, 01/31/94. Because rates fluctuate daily, it is recommended to obtain current rates from local banks or newspapers prior to departure.

\section{DIRECT DIALING}

Individual numbers for direct dial to Switzerland are complete as listed, after dialing international access code: 011. Country code is 41; listed local numbers include city code.

\section{U.S. EMBASSY - BERN}

American Embassy Jubiläumstrasse 93 3001 Bern, Switzerland

Tel: Fax: $\quad 41-31-357-7344$ 
Population

1992

\section{ENERGY}

Electric Power Capacity

$\begin{array}{lll}1992 & 15.5 & \text { GWe } \\ & 19 \% & \text { nuclear } \\ 1995 & 15.8 & \text { GWe } \\ & 20 \% & \text { nuclear } \\ 2000 & 17.0 & \text { GWe } \\ & 19 \% & \text { nuclear }\end{array}$

Electric Power Production 1992
57.8 TWh

$57 \%$ hydro/geoth.

$41 \%$ nuclear

$1 \%$ oil

$1 \%$ other

1995

$39 \%$ nuclear

$36 \%$ nuclear

\section{NUCLEAR POWER}

Pollcy: Federal government is in favor of nuclear power, but local opposition has delayed its expansion.

Nuclear Power Capacity

Reactor Mix
1992

1994

\section{$3.0 \mathrm{GWe}$}

3.0 GWe

BWR $2(1972 / 84)$

PWR 3 (1969-79)

\section{INDUSTRIAL FUEL CYCLE}

Pollcy: Purchase most services from other countries, including reprocessing of spent fuels; recycle Pn to LWRs or FBRs.

Waste Management Stralegy: Develop two waste repositories: a horizontally accessed rock cavern in a host rock with considerable overburden for LLW/LLW, and a deep repository in crystalline rock or sedimentary formations for HLW glass and unreprocessed SF elements and alpha wastes; interim storage of all waste at common center nntil repositories available; ocean-dnmping of LLW discontinued in 1982. 
Cumulative SF

Arisings (LWR)

Cumulative Waste Arisings* LLW/

(Planning basis: after D\&D W 135,000 or $135,000 \mathrm{~m}^{3}$

40-yr operation at $3 \mathrm{GWe}$ ) LLW/LW 15,000 or $10,000 \mathrm{~m}^{3}$ HLW glass $\quad 500$ or $160 \mathrm{~m}^{3}$

* Two scenarios considered: complete reprocessing (left column) or no reprocessing (right column) after the year 2000.

\section{Major Milestones}

- Initial receipt of HLW glass from COGEMA (France) >1996

- Intermediate-depth repository for LLW/LW

- Geologic repository for HLW, SF, and alpha wastes

\section{INTERNATIONAL RELATIONSHIPS}

DOE/NAGRA Agreement for Cooperation in Radioactive Waste Management

Term: $04-19-85$ to $09-22-96$

Scope: Preparation and packaging of wastes; field and laboratory testing; storage; geologic disposal; environment and safety, design and operational issues; transportation requirements; public acceptance issues; information exchange and direct cooperation, particularly concerning Grimsel Pass URL activities.

Member of IAEA and OECD/NEA; cooperative agreements with SKB/Sweden, CEA/France, Euratom/EC, ONDRAF/Belgium, PNC/Japan, BfS, BMFT, GSF, and BGR/Germany, TVO/Finland. 


\section{ORGANIZATION}

- Nagra--National Cooperative for the Disposal of Radioactive Waste-formed by utilities/government to handle fuel cycle/waste management activities.

- PSI--Paul Scherrer Institut--formed (1987) through merger of EIR (Federal Institute for Reactor Research) and SIN (Swiss Institute for Nuclear Research).

- Federal Energy Omce--sets criteria for waste management practices, including geologic disposal.

\section{BEW (Federal Omice for Energy)}

Bundesamt für Energiewirtschaft Nuclear Safety Inspectorate (HSK) 5232 Villingen, Switzerland Tel: $\quad 41-56-98-28-53$ Fax: $\quad 41-56-99-39-07$

Waste Management Section Dr. Auguste Zurkinden

Function: Licensing and inspection of nuclear installations. 
NAGRA/CEDRA/CISRA (National Cooperative for the Disposal of Radioactive Waste)

Nationale Genossenschaft für die Lagerung

radioaktiver Abfälle (Nagra)

or

Société coopérative nationale pour

l'entreposage de déchets radioactifs (Códra)

or

Società cooperativa nazionale per

l'immagazzinamento di scorie radioattive (Cisra)

Hardstrasse 73

5430 Wettingen, Swilzerland

President

Director, Science/Technology

Geology

Field Operations/Testing

Engineering

Nuclear Technology

Director, Repository Projects
Tel:

41-56-37-11-11

Fax: 41-56-37-12-07

Dr. Hans Issler

Dr. Charles McCombie

Dr. Marc F. Thury

Dr. Ch. Sprecher

Andreas L. Nold

Dr. Piet Zuidema

Dr. Emil Kowalski

Function: Provide for safe disposal of radioactive wastes produced by the Swiss nuclear industry; funded by utilities and government.

\section{Facilities}

- URL at Grimsel Pass-operational since 1984 (tests/experiments in crystalline rock). 


\section{PSI}

Paul Scherrer Institut

Würenlingen/Villigen

5232 Villigen, Swilzerland

Tel: $\quad 41-56-99-2111$

Fax: $\quad 41-56-98-2327$

Director

Manager, Waste Mgmt. Proj.

Prof. Meinrad Eberie

Dr. J. Hadermann

Function: Federal (Department of Interior) institute for reactor and nuclear R\&D.

Waste Management R\&D: Incineration of TRU wastes; modeling of radionuclide migration through heterogeneous geologic media; chemical behavior of radionuclides during migration; transport of radionuclides through the biosphere; natnral analogue studies; hydrological studies; sorption constants on different rocks; immobilization of LLW/ILW in cement; leaching rates on LLW/ILW forms; and long-term corrosion tests on waste package materials.

\section{Facilities}

- Hot Cells, radioactive laboratories, incinerator.

- ADA (Acid Digestion Plant) for TRU wastes.

Design Basis: Carbonization/digestion in $\mathrm{H}_{2} \mathrm{SO}_{4} / \mathrm{HNO}_{3}$ at $150^{\circ} \mathrm{C}$; capacity, $1 \mathrm{~kg} / \mathrm{h}$ solid wastes.

Hislory: Non-Pu runs, late 1981; Pu runs, 1982. 


\section{ZWILAG (Interim Waste Storage Facility)}

Zwischenlager Würenlingen AG

Parkstrasse 23

5401 Baden, Switzerland

Tel: $\quad 41-56-20-31-11$

Director

Fax: $\quad 41-56-20-37-58$

Dr. Hans R. Lutz

Tech. Project Manager

Function: Provide interim storage for spent fuel, HLW, LLW, and ILW; the facility was voter-approved in 1989 and will be managed by the local council and the nuclear utilities; construction is expected to take at least two years (startup in 1995) and to cost about U.S. \$350 million; organization is a consortium of Swiss nuclear utilities. 
TAIWAN

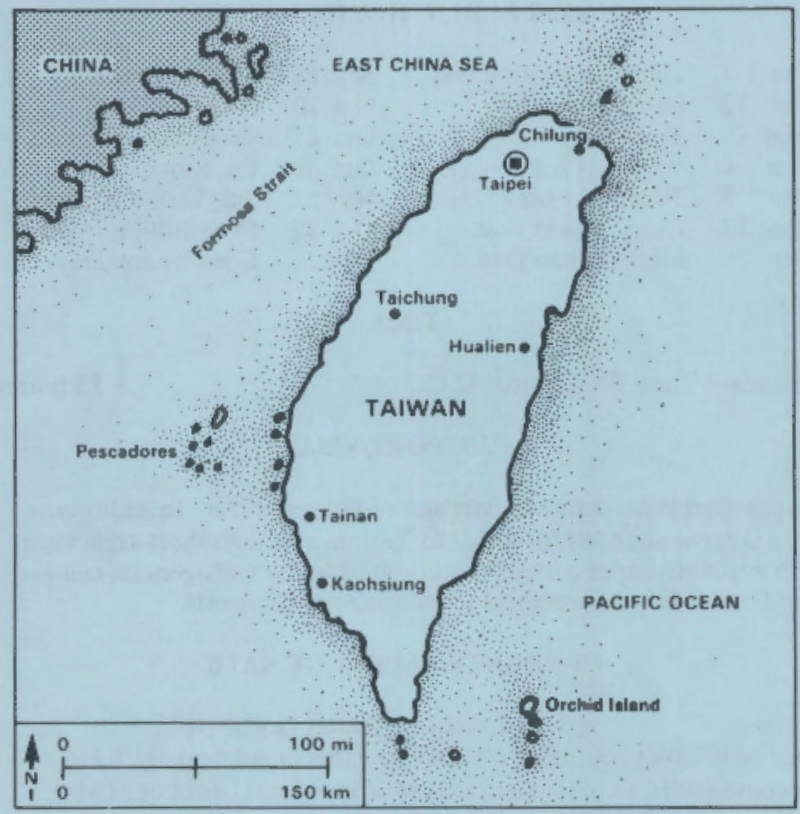




\section{TAIWAN}

MAJOR PUBLIC HOLIDAYS (1994)

$\begin{array}{llll}\text { Jan 1-3 } & \text { Commemoration Day } & \text { Sep 28 } & \text { Confucius Birth } \\ \text { Feb 12 } & \text { Lunar New Year } & \text { Oct 10 } & \text { National Day } \\ \text { Mar 29 } & \text { Youth Day } & \text { Oct 25 } & \text { Taiwan Restoration } \\ \text { Apr 4 } & \text { Women/Children's Day } & \text { Oct 31 } & \text { Ch. Kai-Shek's Birth } \\ \text { Apr 5 } & \text { Tomb Sweeping Day } & \text { Now 12 } & \text { Sun Yat-Sen's Birth } \\ \text { Jun 13 } & \text { Dragon Boat Fest } & \text { Dec 25 } & \text { Constitution Day } \\ \text { Sep 20 } & \text { Mid-Autumn Day } & \text { Dec 26 } & \text { Constitntion Day }\end{array}$

TIME

Standard Time Washington, D.C. +13 hours

\section{PASSPORT/VISA}

A passport is needed to depart and re-enter the U.S. In addition, a visa is recommended for a visit to Taiwan, although short-term visas are available under certain conditions. Most travel agencies can provide up-to-date information concerning requirements.

\section{CURRENCY EXCHANGE RATE}

1 U.S. $S=26.42$ Tiwan Dollar per Wall Street Journal, 01/31/94. As rates fluctuate daily, it is recommended to obtain current rates from local banks or newspapers prior to departure.

\section{DIRECT DIALING}

Individual numbers for direct dial to Taiwan are complete as listed, after dialing international access code: 011 . Country code is 886; listed local numbers include city code.

\section{AIT - TAIPEI}

American Institute in Taiwan

7 Lane 134

Hsin Yi Road, Sec. 3

Tel: $\quad 886-2-709-2000$

Taipei, Taiwan

Fax: $\quad 886-2-702-7675$

Science Officer

Della Knox-Bennett 
Population

ENERGY
1993

21 million

\section{Electric Power Capacity \\ Electric Power Production \\ 1993 \\ $19.3 \mathrm{GWe}$ $27 \%$ nuclear \\ Electric Power Production \\ 1993

105.8 TWh
$35 \%$ coal
$32 \%$ nuclear
$16 \%$ oil
$10 \%$ other
$7 \%$ hydro

\section{NUCLEAR POWER}

Policy: Plan for nuclear power to meet rapidly growing demand for electric energy; continue with nuclear power at about $1 / 3$ of total electricity.

$\begin{array}{lcc}\text { Nuclear Power Plant Capacity } & 1993 & 4.9 \mathrm{GWe} \\ & 1995 & 4.9 \mathrm{GWe} \\ & 2000 & 6.7 \mathrm{GWe} \\ & 1993 & \text { BWR } 4(1978-83) \\ \text { Reactor Mix } & & \text { PWR } 2(1984 / 85)\end{array}$

\section{INDUSTRIAL FUEL CYCLE}

Policy: Purchase fuel materials and enrichment; develop indigenous fuel production capability: $\mathrm{UF}_{6}$ conversion; $\mathrm{UO}_{2}$ pellet preparation; fuel hardware fabrication; fuel assembly.

Wasle Management Strategy: Evaluating spent fuel/HLW interim storage options; may reprocess (in other countries); maximize existing SF pool storage capacity by reracking; build MRS facility at existing reactor site for interim storage until final disposal in geologic repository; LLW stored in National Waste Storage Facility on nearby Orchid Island; LLW/ILW will eventually be disposed of on the sea floor, if internationally acceptable, or in a shallow-land facility. 
Cumulative SF Arisings (LWR) $\quad 1990 \quad 1,140$ tU

$$
2000 \quad 2,600 \mathrm{tU}
$$

\section{Major Milestones}

LLW Disposal Facility

- Selection of site/method

- Site characterization, engineering, design and licensing 1998

- Completion, start of operation

HLW Disposal Facilities

- Commission MRS at Chinshan reactor site 1999

- Geologic repository site selection 2016

- Commission final repository 2032

\section{ORGANIZATION}

- TAIPOWER (Taiwan Power Company) - operation of nuclear power plants (owned by the government); country's only electric utility.

- AEC (Atomic Energy Council) - regulatory functions; RWA (Radwaste Administration) - radwaste disposal.

- INER (Institute of Nuclear Energy Research) - nuclear R\&D. 
Atomic Energy Council

67, Lane 144

Keelung Road, Section 4

Taipei 10772, Taiwan

Chairman

Sr. Vice Chairman

Vice Chairman

Director, Radwaste Admin.

Dir., Planning

Tel: $\quad 886-2-363-4180$

Fax: $\quad 886-2-363-5377$

Dr. Y. Y. Hsu

Prof. Thomas K.C. Liu

Dr. Adrian M. C. Wang

Dr. Chao-Ming Tsai

Tel: $\quad 886-2-964-7401$

Fax: $\quad 886-2-964-7464$

S. T. Chiou

Dir., Rad. Protection

$886-2-362-8567$

W. L. Chen

886-2-362-6189

Dir., Nuc. Regulations

T. T. Huang

886-2-362-8571

Dir., Nuc. Technology

Yi-Bin Chen

\section{INER}

Institute of Nuclear Energy

Research

P.O. Box 3

Lung-Tan 32500, Taiwan

Director

Dep. Directors

Nuc. Engineering

Nuc. Instrumentation

Nuc. Fuel/Matls. Research

Health Physics

Tel: $\quad 886-2-365-1717$

Fax: $\quad 886-2-471-1064$

Dr. Der-Yu Hsia

Dr. Song-Feng Wang

Dr. Ging-Shung Yu

Dr. Gang Ting

Dr. Yuan-Ching Chou

Dr. D. S. Lee

Dr. Li-Fu Lin

Dr. S. T. Su

Fuel Cycle R\&D: Solvent exiraction technology; yellowcake conversion to $\mathrm{UO}_{2}$; cement and thermoplastic waste forms for reactor wastes; HLW conditioning processes; burial of LLW. 
TAIWAN

PNL-9450-1

\section{TAIPOWER}

Taiwan Power Company

17F, 242 Roosevelt Rd., Sec. 3

Taipei 107, Taiwan

Tel: $\quad 886-2-365-1234$

Fax: $\quad 886-2-396-8593$

Chairman

C. C. Chang

President

S. M. Chang

Vice President

M. C. Tsai

Dir., Nuc. Engineering

Victor Y.C. Liao

886-2-367-7969

Dir., Nuc. Operation

S. J. Tsuei

886-2-367-7126

Dir., Nuc. Safety

A. H. Jeng

886-2-367-7341 


\section{UNITED KINGDOM}

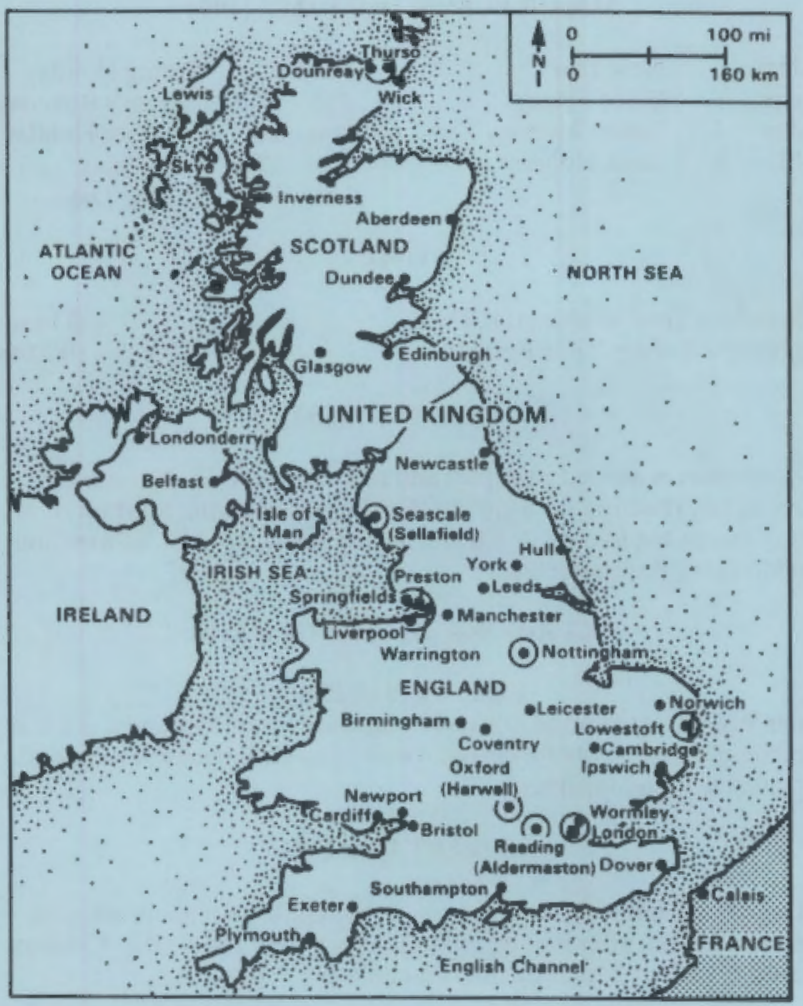




\section{UNITED KINGDOM}

\section{MAJOR PUBLIC HOLIDAYS (1994)}

$\begin{array}{lll}\text { Jan } & 1 & \text { New Year } \\ \text { Apr } & 1 & \text { Good Friday } \\ \text { Apr } & 4 & \text { Easter Monday } \\ \text { May } & 2 & \text { Bank Holiday }\end{array}$
May 23 Spring Holiday
Jun 12 Queen's Birthday
Aug 29 Summer Holiday
Dec 25 Christmas
Dec 26 Boxing Day

\section{TIME}

Standard Time Washington, D.C.

Daylight Savings Time Period:

\section{PASSPORT/VISA}

A passport is needed to depart and re-enter the U.S. A visa is currently not required for a visit to the United Kingdom; however, it is recommended to consult a travel agency for up-to-date information concerning requirements.

\section{CURRENCY EXCHANGE RATE}

1 U.S. $\$=0.66$ Pound

per Wall Street Journal, 01/31/94. Because rates fluctuate daily, it is recommended to obtain current rates from local banks or newspapers prior to departure.

\section{DIRECT DIALING}

Individual numbers for direct dial to the United Kingdom are complete as listed, after dialing international access code: 011. Country code is 44; listed local numbers include city code.

\section{U.S. EMBASSY - LONDON}

American Embassy

24/31 Grosvenor Square

London W1A 1AE, United Kingdom

Science Counselor
Tel: $\quad 44-71-499-9000$

Fax: $\quad 44-71-409-1637$

Jeffrey T. Lutz 
PNL-9450-1

Population

1992

60 million

\section{ENERGY}

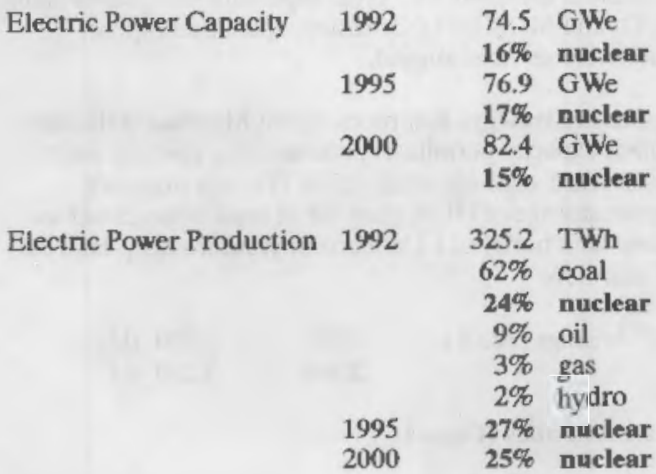

\section{NUCLEAR POWER}

Policy: Continue nuclear power as a significant element of total electricity production; substantially based, to date, on gas-cooled reactors, but now diversifying to PWRs; eventual active FBR pursuit expected.

$\begin{array}{lcrll}\text { Nuclear Power Capacity } & 1992 & 12.0 & \text { GWe } \\ & 1995 & 13.0 & \text { GWe } \\ & 2000 & 12.0 & \text { GWe } \\ \text { Reactor Mix } & 1993 & \text { GCR } & 20 & (1956-72) \\ & & \text { AGR } & 14(1976-89) \\ & & \text { FBR } & 1(1976) \\ & & \text { PWR } & 1(1994)\end{array}$

Reactor Development PWRs; future LMFBR development. 


\section{INDUSTRIAL FUEL CYCLE}

Policy: Reprocess and recycle U to AGR and LWR systems; develop and maintain complete fuel cycle capability $\left(\mathrm{UF}_{6}\right.$ conversion, enrichment, $\mathrm{UO}_{2}$ and $\mathrm{MOX}$ fuel fabrication, spent fuel reprocessing); sell fuel cycle services abroad.

Waste Management Strategy: Reprocess spent Magnox/AGR fuels as rapidly as plant capacity permits; reprocess other thermal reactor fuel after several years' cooling; vitrify HLW (French process); long-term interim storage of HLW glass for at least 50 years before disposal; shallow-land burial of LLW currently; future deep-land disposal of LLW and ILW.

$\begin{array}{lll}\text { Cumulative SF Arisings (AGR) } & 1990 & 1,300 \mathrm{tU} \\ & 2000 & 3,250 \mathrm{tU}\end{array}$

\section{Industrial-Scale Activities (Capacity)}

- Uranium conversion (Springfields)

- $\mathrm{UF}_{6}$ production: $9,000 \mathrm{~V} / \mathrm{a}$

- $\mathrm{UO}_{2}$ conversion: $10,000 \mathrm{~L} / \mathrm{a}$

- Uranium enrichment (Capenhurst)

- centrifuge plant: 800 tSWU/a

- Fuel fabrication

- Springfields

U metal (Magnox): 1,300 tU/a

AGR/LWR fuels: $300 \mathrm{t} / \mathrm{a}$

- Sellafield

- MOX fuels capacity, 1992: 6 t/a (LWR)

- Fuel reprocessing

- Magnox fuels (Sellafield): up to $1500 \mathrm{ta}$

- UO ${ }_{2}$ fuels (THORP," ): $1200 \mathrm{t} / \mathrm{a}$ (1992)

- FBR fuels (PFR, Dounreay): $\quad 50 \mathrm{kgHM} / \mathrm{d}$

- HLW vitrification

- Sellafield Vitrification Plant, radioactive operation, 1990 


\section{INTERNATIONAL RELATIONSHIPS}

\section{DOEJUKAFA Agreement in the Fleld of Decommtssioning Nuclear Facilities}

Term: $03-01-85$ to $03-01-93$

Scope: Techniques used, schedules, costs, manpower, radiation exposures, and waste arisings relevant to decommissioning projects (U.S./Shippingport Station - U.K./Windscale AGR); treatment, packaging, storage, transportation, disposal methods, and costs for wastes arising from the decommissioning operations; emphasis on exchange of technical information, specialists, samples, materials, instruments, and testing equipment.

\section{DOE/UKAEA Agreement in the Field of Radioactlve Waste Management Technology}

Term: $\quad 10-30-86$ to $10-29-91$

Scope: LLW/LW, TRU waste and D\&D technology; treatment; geologic disposal; transportation; storage; environment/ safety and public acceptance issues; performance assessment; packaging; emphasis on technical information exchange, primarily TRU waste treatment.

Member of EC, IAEA and OECD/NEA; agreements/partnerships with various nations. 


\section{ORGANIZATION}

- AEA Technology: nuclear research; laboratories at Harwell, Risley, Sellafield, Springfields, Dounreay.

- DoE (Department of Environment): develops waste management strategy, funds and coordinates generic waste management R\&D.

HMIP (Her Majesty's Inspectorate of Pollution): regulation of effluent discharges to the environment.

- BNFL (British Nuclear Fuels plc): commercial fuel cycle and engineering services for domestic and foreign customers.

- NIREX ("private limited"/government-owned company): LLW and ILW disposal in deep repository.

- BGS and IOS (British Geological Survey and Institute of Oceanographic Sciences): supporting R\&D for the waste management program.

- NRPB (National Radiological Protection Board): environmental R\&D.

- NII (Nuclear Installations Inspectorate): licensing.

- MAFF (Ministry of Agriculture, Fisheries and Food): regulation of waste management. 


\section{NUCLEAR FUEL CYCLE/WASTE MANAGEMENT} ORGANIZATION

National Government

Department of Environment (DoE)

1 H.M. Inspectorate of Pollution (HMIP)

- Radioactive WM Advisory Committee (RWMAC)

- Building Research Establishment (BRE)

Department of Health/Soclal Services

- National Radiological Protection Board (NRPB)

Department of Trade and Industry (DTI)

- Nat. Environment Research Council (NERC)

- British Geological Survey (BGS)

- Inst.of Oceanographic Sciences (IOS)

- Department of Energy (DEN)

- Nuclear Electricity Authorities

- NIREX

- British Nuclear Fuels plc (BNFL)

- AEA Technology

- Health and Safety Executive (HSE)

- Nuclear Installations Inspectorale (NII)

- Ministry of Defense (MOD)

- Alomlc Weapons Res. Establlshment (AWE)

- Ministry of Agriculture, Fisheries and Food (MAFF)

- Fisheries Laboratories 


\section{FUEL CYCLE/WASTE MANAGEMENT RESPONSIBILITIES}

Department of Energy (DEN)

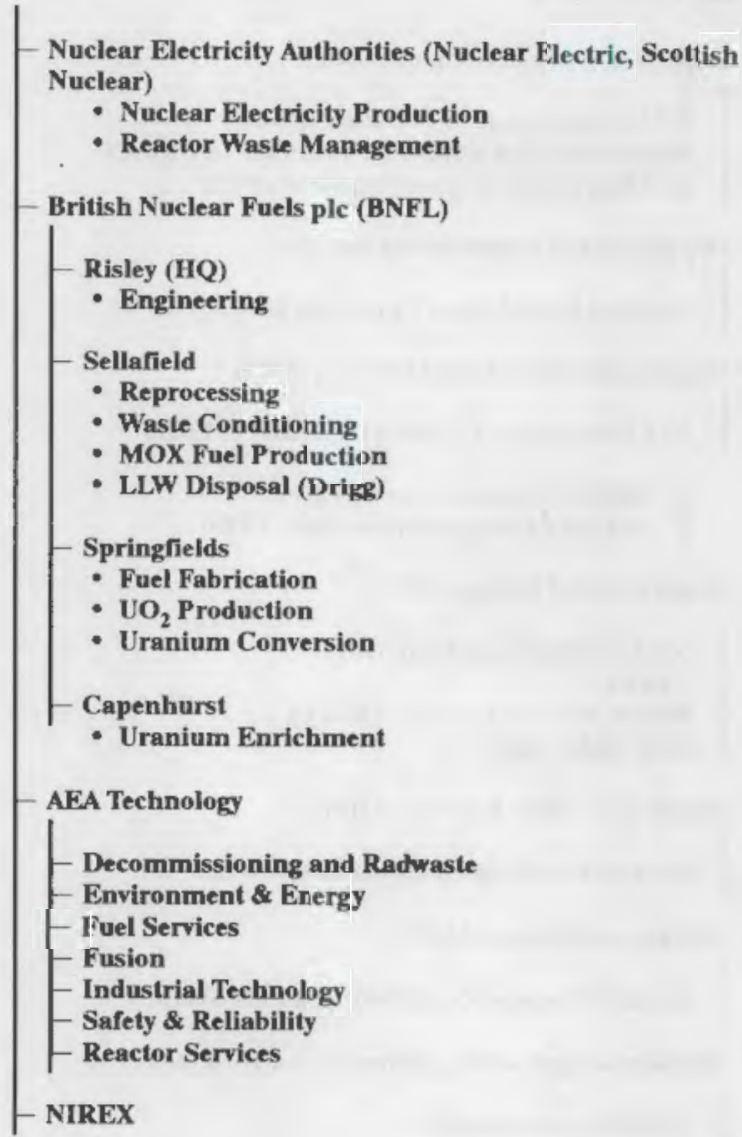




\section{AEA}

AEA Technology

Corporate Headquarters

11 Charles II Street

London SW1Y 4QP

United Kingdom

Tel: $\quad 44-71-389-6565$

Fax: 44-71-389-6841

Chairman

Dep. Chairman/Chief Exec.

Managing Dir., Nuc. Bus. Group

Managing Dir., Ind. Bus. Group

Managing Dir., Site Operations

Sir Anthony Cleaver

Brian L. Eyre

Dr. Derek Pooley

R. Stuart Nelson

Graeme G. E. Low

Chief Technologist, Nuclear

Dr. Ron H. Flowers

Government-owned nuclear research and applications agency, since 1986 operating on a fully commercial basis; provides contract R\&D, technical and engineering services to governments and companies in the U.K. and worldwide.

\section{ÁEA D\&R}

AEA Decommissioning and

Radwaste

Winfrith Technology Center

Dorchester, Dorset DT2 8DH

United Kingdom

Tel: $\quad 44-305-20-2066$

Fax: $\quad 44-305-20-2500$

Chief Executive

Head, Business Development

Dr. Mel H. Wood

Dr. Ron K. Webster

Activities: Decommissioning of all types of nuclear facilities; all aspects of radioactive waste storage, processing, transport, and disposal; decontamination technology and robotic handling. 


\section{AEA E\&E}

AEA Environment \& Energy

Harwell Laboratory

Oxfordshire OX11 ORA

Tel: $\quad 44-235-43-5530$

United Kingdom

Fax: $\quad 44-235-43-4361$

Chief Executive

Dr. J. Rae

Head, Business Development.

Dr. A. E. J. Eiggleton

Activities: R\&D and consulting services to industry and regulatory bodies covering pollution control technology, waste management, and regional and global environmental impacts.

Facilities

- Harwell Ceramic Melter Test Unit (nonradioactive) Mission: Develop ceramic melter capability for AEA.

Design Basis: Liquid-fed ceramic melter; capacity, $700 \mathrm{~kg} / \mathrm{d}$ glass; product, borosilicate glass.

History: Initial 1/3-scale unit 1982-84; startup (full scale), 1986.

\section{AEA FS}

AEA Fuel Services

AEA Technology Dounreay

Caithness KW14 7TZ

United Kingdom

Tel: $\quad 44-847-6-2121$

Ext. 674

Fax: $\quad 44-847-6-2121$

Ext. 666

Chief Executive

Head, Business Development

Owen Pugh

Dr. Robert Anderson

Activities: Fuel reprocessing, special fuel manufacturing and testing, laser enrichment, waste conditioning, R\&D in radioactive equipment and safeguards.

(contd next page) 


\section{AEA FS (contd)}

\section{Facilities}

- PFR Reprocessing Plant Mission: Reprocess Dounreay Prototype Fast Reactor (MOX) fuels.

Design Basis: Shear single pins and leach; PUREX process; capacity 9-10 tHM/a of 180-day cooled PFR assemblies with 8-10\% burnup.

History: Dounreay fast reactor fuels processed from 1961 to 1975; plant rebuilt to handle PFR oxide fuels, resumed operation in October 1980.

- Solidification Plant

Mlssion: Condition liquid wastes by cementation.

History: startup, 1987 (cost US \$8.84 million).

- Marshall Laboratory (Fuel-processing research, opened in 1986).

\section{AEA FUSION}

Culham Laboratory

Culham, Abingdon

Oxfordshire OX14 3DB

Tel: $\quad 44-235-46-3556$

United Kingdom

Fax: $\quad 44-235-46-3256$

Chief Executive

Head, Business Development

Dr. D. R. Sweetman

I. M. Pollard

Function: Management of U.K participation in international fusion programs, in particular, the Joint European Torus (JET). 


\section{AEA IT}

AEA Industrial Technology

Harwell Laboratory

Oxfordshire OX11 ORA

United Kingdom

Tel: $\quad 44-235-43-2138$

Chief Executive

Head, Business Development

Fax: $\quad 44-235-43-2064$

Dr. Chris Wright

Dr. Steve J. Curl

Function: Provide advanced technology to the process, manufacturing electronics, defense, and aerospace industries; process technology and plant design; instrumentation and control; materials technology and manufacture; structural assessments; advanced computing; laser applications; and computational fluid dynamics.

\section{AEA RS}

AEA Reactor Services

Risley, Warrington

Cheshire WA3 6AS

United Kingdom

Tel: $\quad 44-925-25-3019$

Fax: $\quad 44-925-25-2196$

Chief Executive

Dr. Tony Broomfield

Head, Business Development

Dr. Neil M. Irvine

Activities: Fast/thermal reactor technology; management of FR program and participation in international FR programs, especially the European FR; design and operational techniques for thermal reactors aimed at improving economies of existing plants and design of new plants. 


\section{AEA S\&R}

AEA Safety and Reliability

Wigshaw Lane, Culcheth

Warrington WA3 6AT

Tel: $\quad 44-925-25-4241$

United Kingdom

Fax: $\quad 44-925-25-4535$

Chief Executive

Dr. Geoff Ballard

Head, Busineas Development

Anthony R. Taig

Function: Safety and reliability analysis and assessment services to government and companies in the nuclear and non-nuclear sectors, including oil and gas, defense contractors, insurance, manufacturing, and engineering companies.

\section{$\underline{\text { AWE }}$}

Atomic Weapons Establishment Aldermaston, Reading RG7 4PR United Kingdom

Tel: 44-73-56-4111

Fax:

Waste Management

S. Hunter

BGS

British Geological Survey

Nicker Hill, Keyworth

Nottingham, NG12 5GG

United Kingdom

Tel: $\quad 44-60-77-6111$

Fax: 44-60-77-6602

Director

Dr. P. J. Cook 


\section{BNFL}

British Nuclear Fuels plc

Risley, Warrington

Cheshire WA3 6AS

Tel: $\quad$ 44-925-83-2502

United Kingdom

Fax: $\quad 44-925-82-2711$

Ver: $\quad 44-925-83-2369$

Location: About 20 miles by car from Manchester International Airport, or by train from London to Warrington (apprax. 3 hours), then 6 miles by car to Risley.

Chainnan

Chief Exec. Officer

Dep. Chief Exec. Officer

Dir., International Group

Dir., Engineering Group

Dir., U.K. Group

BNFL, lne.

1776 I Street NW

Washington, DC 20006

President
John Guiness

44-925-83-5000

N. L. Chamberlain

44-925-83-5006

Dr. Greg G. Butler

Graham Watts

Ken G. Jackson

Graham Smith

Tel: $\quad$ 202-785-2635

Fax: $\quad 202-785-4037$

R. "Landy" Langley

\section{BNFL: CAPENHURST}

British Nuclear Fuels plc

Capenhurst Works

CHESTER

Cheshire CH1 6ER

United Kingdom

Tel: $\quad$ 44-51-339-4101

Fax: 44-51-339-5541

Dir., Enrichment Division

Dr. Peter C.. Upson

Function: Enrichment of $U$ by centrifuge process (URENCO). 


\section{BNFL: SELLAFIELD}

British Nuclear Fuels plc

Sellafield, Seascale

Cumbria CA20 1PG

United Kingdom

Tel: $\quad 44-9402-8333$

Fax: $\quad 44-9467-28987$

Location: From London-Euston Station to Carlisle Station by train ca. 4 hours; transport can be arranged by BNFL from Carlisle to site (approx. 1-1/2 hours; from Manchester International Airport to site by car is ca. 3 hours.

Dir., Magnox Reprocessing

Dir., THORP Division

Dir., Waste Mgmt./Decom. Div.

Dir., Reactor Division
Grahame K. Smith

Dave Bonser

44-9402-74245

A. D. Evans

Function: Provide spent fuel management services, including storage, reprocessing and waste management; transport of SF/wastes and complete fuel cycle services.

\section{Facilities}

- B205 (Magnox Fuel Reprocessing Plant)

Mission: Reprocess Magnox (magnesium-clad, U metal) fuels from U.K. GCRs.

Design Basis: Magnox fueis--mechanical declad; PUREX flowsheet; "no-maintenance" concept; nominal capacity, $1500 \mathrm{t} / \mathrm{a}$; HLLW storage--SS tanks, $70 \mathrm{~m}^{3}$ and $150 \mathrm{~m}^{3}$, in SS-lined concrete cells.

History: Magnox fuels--B205 startup, 1964; annual throughput of Magnox fuels, 1000-1200 tHM; oxide head-end (installed in B204), operated 1969-1973 and processed 90 toxide fuel; shut down after a contamination release incident.

- Magnox Fuel Handling Plant

- Storage/decanning of Magnox fuel.

- Storage/dismantling of AGR fuel.

(contd next page) 


\section{BNFL: SELLAFIELD (contd)}

- THORP (Thermal Oxide Reprocessing Plant)

Mission: Reprocess AGR, domestic and foreign LWR fueis.

Design Basis: PUREX flowsheet, pulsed columns and mixer-settlers; "no maintenance" concept; nominal capacity, 1200 tU/a.

History: Startup, 2/1994.

- Drigg Waste Disposal Facility (300-acre site, 4 miles from Sellafield) Mission: LLW disposal.

Design Basis: Shallow-land disposal in clay-based trenches and recently, in concrete vaults.

Capacity: $650,000 \mathrm{~m}^{3} \mathrm{LLW}$ disposed of through 1989 .

- MOX Fuel Fabrication Facilities

- Pilot plant, capacity--7 t/a

- Production plants, capacity--120 t/a; startup, 1995.

- Vitrification Plant

Mission: Solidify Sellafield HLW.

Design Basis: AVM process; product, borosilicate glass blocks.

Capacity: $250-300$ t/a glass.

History: Startup, 1990.

- WTC (Waste Treatment Complex)

Mission: Prepare TRU waste for disposal; underground refurbishment to include supercompaction.

Milestone: Startup, 1995

- EP-1 and EP-2

Mission: Encapsulate ILW in cement matrix in 500-liter drums.

Capacity: 13 500-liter drums/d (EP-1); 20 500-1 drums/d (EP-2).

History: Startup EP-1, 1990.

Milestone: Startup EP-2, 1994.

- EARP (Enhanced Actinide Removal Plant)

Mission: Remove actinides from liquid effluents by ultra-

filtration and flocculation.

Capacity: $1000 \mathrm{~m}^{3} / \mathrm{d}$.

Milestone: Startup, 1994. 


\section{BNFL: SPRINGFIELDS}

British Nuclear Fuels plc

Springfields Works

Salwick, Preston

Lancashire PR4 OXJ

United Kingdom

Tel: $\quad 44-772-72-8262$

Fax: $\quad 44-772-72-5607$

Director, Fuel Division

Dr. R. Smith

Function: Supply fuel for U.K. reactors; $\mathrm{UF}_{6}$ conversion to $\mathrm{UO}_{2}$ powder/pellets production; fabricate PWR fuel; provide recycle services (enrichment in conjunction with URENCO).

\section{BRE}

Building Research Establishment

Department of the Environment

Building Research Station

Garston, Watford WD2 7JR

United Kingdom

Tel: $\quad 44-927-894040$

Fax:

Asst. Dir., Geotech./Struc. Eng.

Seabed Disposal

Dr. J. B. Menzies

Continental Disposal

T. Freeman

Geotech. Division

Ms. C. M. Cooling

R. M. C. Driscoll

Waste Management R\&D: Emplacement engineering and related activities; rock mechanics. 


\section{HMIP}

H.M. Inspectorate of Pollution

Department of the Environment

Romney House, 43 Marsham Street

London SWIP 3PY

United Kingdom

Tel: $\quad 44-71-276-3000$

Fax: $\quad 44-71-276-8100$

Chief Executive

Dr. David Slater

Director, Regulatory Systems Div.

44-71-276-8080

Dr. Alan Duncan

44-71-276-8129

Research

Dr. Steven Brown

Waste Management Responsibility: Administer U.K. waste management programs; fund and coordinate waste treatment and waste isolation R\&D at Harwell, BGS, and NRPB; regulate discharge of radioactive materials to the environment.

\section{$\underline{\text { IOS }}$}

Institute of Oceanographic Sciences Brook Road, Wormley, Godalming Surrey GU8 5UB United Kingdom

Tel: $\quad 44-42-879-4141$

Fax:

Director

Dr. C. Summershayes

Function: Model radionuclide transport in the ocean. 


\section{MAFF}

Ministry of Agriculture,

Fisheries and Food

Ergon House, Room 231

c/o Nobel House

17 Smith Square

London SW1P 3JR, U.K.

Tel: $\quad 44-71-238-6170$

Fax: $\quad 44-71-238-6215$

Chief Inspector

J. C. Sherlock

Function: Regulate, jointly with HMIP, management of waste prior to disposal.

MAFF Fisheries Laboratory

Pakefield Road

Lowestoft, Suffolk NR33 OHT

United Kingdom

Tel: $\quad 44-502-562244$

Director, Research

Fax:

D. J. Garrod

\section{NII}

Nuclear Installations Inspectorate

Baynards House

1 Chepstow Place

Westbourne Grove

London W2 4TF, U.K.

Tel: $\quad 44-71-243-6000$

Fax: $\quad 44-71-727-4116$

Chief Inspector/Nuc.Installations

Sam Harbison

Function: Licensing of nuclear facilities. 


\section{NIREX}

U.K. Nirex Ltd.

Curie Avenue, Harwell

Tel: $\quad 44-235-83-5153 /$

Didcot, Oxon OX11 ORH

United Kingdom

Fax: $\quad 44-235-83-1239$

Managing Director

Technical/Projects

P. Tom Mclnerney

H. Beale

Function: Commission/manage research and development to propose (to the government) a site suitable for a deep repository for LLW/ILW; construct and operate the repository; continue necessary $\mathrm{R} \& \mathrm{D}$ on long-term waste emplacement.

Owners: BNFL (42.5\%), Nuclear Electric plc (42.5\%), Scottish Nuclear Ltd. (7.5), and UKAEA (7.5\%) are partners in the "private limited" company. One special share, having absolute power of veto, is held by the Secretary of State for Energy.

\section{NRPB}

National Radiological

Protection Board

Chilton Didcot

Oxfordshire OX11 ORQ

Tel: $\quad 44-235-83-1600$

United Kingdom

Fax: 44-235-83-3891

Director

Dr. Roger H. Clarke

Secretary

G. A. M. Webb

Asst. Dir., Environ. Sci.

Asst. Dir., Physical Sci.

B. Holliday

Asst. Dir., Medical Sci.

Dr. J. A. Dennis

Dr. B. H. MacGibbon

Function: Independent board, established in 1970 as a result of the Radiological Protection Act, members appointed by the Health Ministry; advice to governmental/industrial organizations on radiological protection matters and standards; contract research to improve radiological protection; some technical services. 


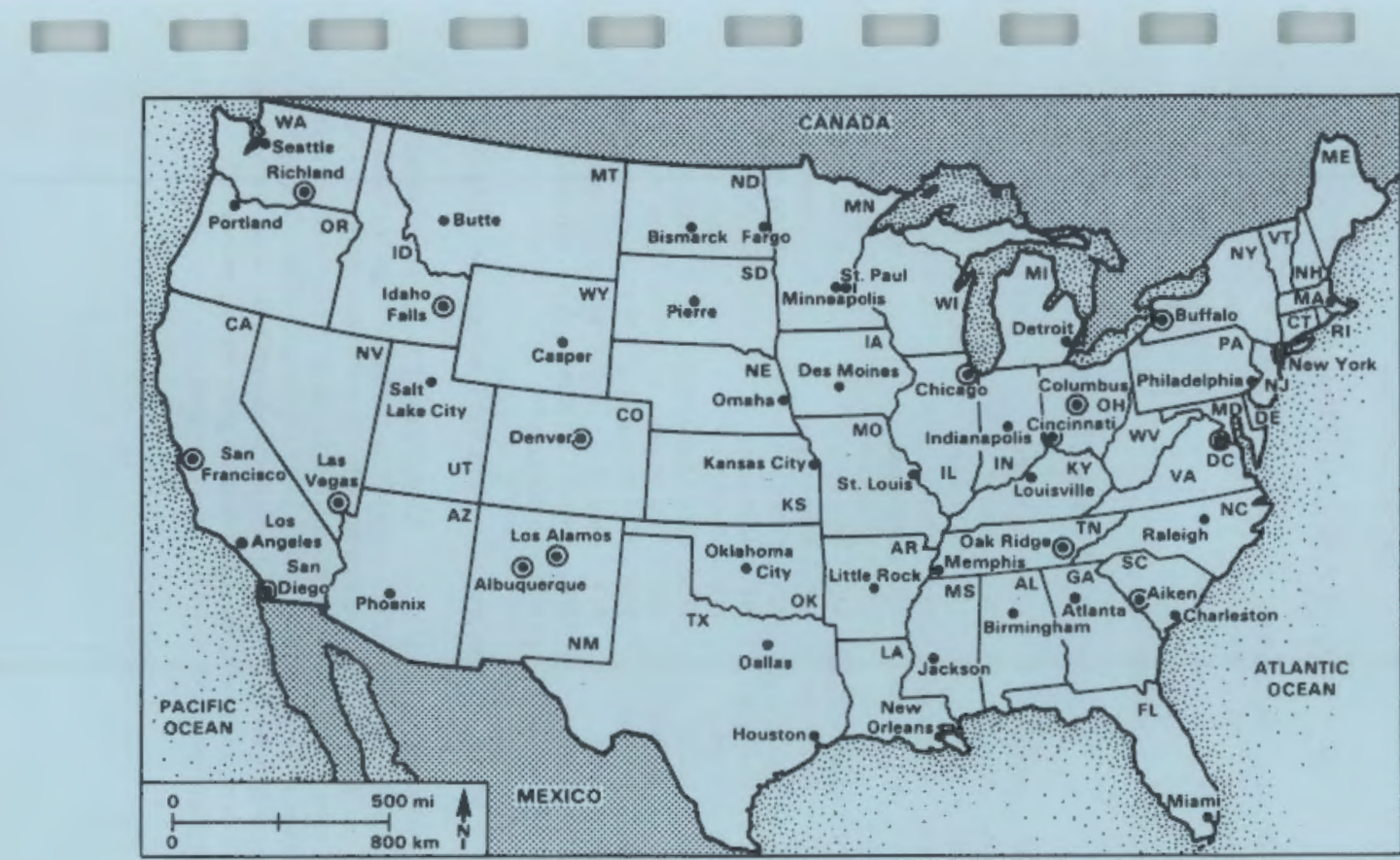

륵 


\section{UNITED STATES}

\section{MAJOR PUBLIC HOLIDAYS (1994)}

$\begin{array}{ll}\text { Jan. 1 } & \text { New Year } \\ \text { Jan. 17 } & \text { M. L. King Day } \\ \text { Feb. 21 } & \text { Presidents Day } \\ \text { May 30 } & \text { Memorial Day } \\ \text { July 4 } & \text { Independence }\end{array}$

$\begin{array}{ll}\text { Sep. } 5 & \text { Labor Day } \\ \text { Oct. 10 } & \text { Columbus Day } \\ \text { Nov. 11 } & \text { Veterans Day } \\ \text { Nov. 24 } & \text { Thanksgiving } \\ \text { Dec. 25 } & \text { Christmas }\end{array}$

Sep. 5 Labor Day

Oct. 10 Columbus Day

Nov. 24 Thanksgiving

Dec. 25 Christmas

STATE ABBREVIATIONS
AL - Alabama
LA - Louisiana
$\mathrm{OH}$ - Ohio
AK - Alaska
ME - Maine
OK - Oklahoma
AZ - Arizona
MD - Maryland
OR - Oregon
AR - Arkansas
MA - Massachusetts
PA - Pennsylvania
CA - California
MI - Michigan
RI - Rhode Island
CO - Colorado
MN - Minnesota
SC - South Carolina
CT - Connecticut
MS - Mississippi
SD - South Dakota
DE - Delaware
MO - Missouri
TN - Tennessee
FL - Florida
MT - Montana
TX - Texas
GA - Georgia
NE - Nebraska
UT - Utah
HI - Hawaii
NV - Nevada
VT - Vermont
ID - Idaho
NH - New Hampshire
VA - Virginia
IL - Illinois
NJ - New Jersey
WA - Washington
IN - Indiana
NM - New Mexico
WV - West Virginia
LA - Iowa
NY - New York
WI - Wisconsin
NC - North Carolina
WY - Wyoming
KY - Kentucky
ND - North Dakota

\section{FOREIGN NATIONAL VISITS TO U.S. DOE FACILITIES}

Foreign visitors to U.S. DOE facilities must complete and submit an IA-473 form (OMB 1910-2100) "Request for Foreign National Unclassified Visit or Assignment" to the laboratory, contractor, or site to be visited at least 30 days before the proposed visit. In certain cases, in lieu of form IA-473, a DOE Operations Office (site specific) form may be used but must be submitted within the same time frame. The request to visit must be based on prior arrangements with appropriate DOE or DOE contractor staff. 
Population

1990

248 million

\section{ENERGY}

$\begin{array}{lcrl}\text { Electric Power Capacity } & 1992 & 740 & \text { GWe } \\ & & 13 \% & \text { nuclear } \\ & 1995 & 755 & \text { GWe } \\ & & 13 \% & \text { nuclear } \\ & 2000 & 789 & \text { GWe } \\ & 13 \% & \text { nuclear } \\ \text { Electric Power Production } & 1992 & 3271 & \text { TWh } \\ & & 53 \% & \text { coal } \\ & & 20 \% & \text { nuclear } \\ & & 13 \% & \text { gas } \\ & & 9 \% & \text { hydro/geotherm } \\ & & 3 \% & \text { oil } \\ & & 19 \% & \text { nuclear } \\ & & 18 \% & \text { nuclear }\end{array}$

\section{NUCLEAR POWER}

Policy: Construction and operation of nuclear power stations is by private and public utilities under close regulatory control by the Nuclear Regulatory Commission (NRC) and state Pnblic Review Commissions; R\&D emphasizes increased inherent LWR safety and small, modular reactor concepts.

$\begin{array}{lcrl}\text { Nuclear Power Capacity } & 1992 & 99 & \text { GWe } \\ & 1995 & 101 & \text { GWe } \\ & 2000 & 104 & \text { GWe } \\ & & & \\ \text { Reactor Mix } & 1993 & \text { PWR } & 71(1970-90) \\ & & & 2(1994) \\ & & \text { BWR } & 3 ?(1969-90)\end{array}$




\section{NUCLEAR FUEL CYCLE}

Policy: Current U.S. commercial nuclear fuel cycle activities include all phases: uranium mining, milling, and enrichment; fuel fabrication; interim spent fuel and waste storage; transportation, conditioning, and disposal of radioactive waste; spent fuel reprocessing is determined by the nuclear industry, which has elected not to reprocess because of economic considerations; disposal of spent fuel and HLW will be implemented in the future. Mining, milling, fabrication of $\mathrm{UO}_{2}$ fuel, and LLW disposal are done predominantly by private firms; enrichment and $\mathrm{HLW} / \mathrm{spent}$ fuel disposal are the responsibilities of the federal government; a private enrichment enterprise is being staried.

Waste Management Strategy: Disposal of U.S. commercial spent fuel in a geologic repository is planned, after interim storage at reactor sites and possibly after interim storage in a monitored retrievable storage (MRS) facility; small amounts of existing commercial HLW and all defense HLW will be vitrified and disposed of in the SF repository; the Nuclear Waste Policy Act (NWPA) of 1982 and its 1987 amendments (NWPAA) mandate start of spent fuel acceptance in 1998 by the government for eventual disposal; short-lived LLW is disposed of in regional near-surface disposal facilities; states and regional compacts of states are developing new commercial LLW disposal facilities; demonstration of defense transuranic (TRU) waste disposal is planned in a geologic repository in a salt formation.

$\begin{array}{lll}\text { Cumulative Spent Fuel Arisings } & 1993 & 27,800 \text { tIHM } \\ & 1995 & 31,400 \text { tIHM } \\ & 2000 & 40,400 \text { tIHM }\end{array}$




\section{Major Milestones}

- Demonstration start of disposal of defense TRU waste at Waste Isolation Pilot Plant (WIPP)

- Candidate site identified for MRS facility

- States/compacts must have civilian LLW disposal capability or manage their own LLW

- States not having LLW disposal capability must take title to all LLW produced within their state

- Startup of MRS facility with limited SF acceptance

- Start construction of geologic repository for commercial SF/HLW

- Startup of repository for spent fuel and HLW

- Start of study on need for second repasitory for SF/HLW

- Environmental cleanup of DOE sites complete

\section{INTERNATIONAL RELATIONSHIPS}

Member of OECD/NEA and IAEA. Bilateral agreements for cooperation (extension of several agreements in process) Canada, CEC, China, Germany, France, Japan, Spain, Sweden, Switzerland, Russia and the U.K; a brief outline of DOE agreements, primarily related to waste management, is provided in the appropriate section of other countries in this report. International cooperation and exchange of waste management technology is encouraged. 


\section{ORGANIZATION}

- DOE (Department of Energy) - Responsible for planning and implementing programs for the safe handling of raclioactive wastes generated by its federal activities and for disposal of all HLW, SF, TRU waste, and greater-than-class-C LLW; responsible for ensuring availability of adequate technology for safe and efficient management of nuclear wastes from both civilian and federal activities.

- HQ (Headquarters) - Provides policy, guidance, and funding for nuclear waste management, including environmental restoration and fuel cycle programs. Specific responsibilities are divided among the following offices:

- EM (Office of Environmental Management) - Environmental cleanup, compliance, technology development, transportation, and waste management activities for DOE sites identified in the Environmental Restoration \& Waste Management FiveYear Plan.

- RW (Office of Civilian Radioactive Waste Management) After-reactor interim storage, transportation, and disposal of spent nuclear fuel and HLW; development of an MRS facility.

- PO (Office of International Research and Development Policy, Assistant Secretary for Policy, Planning and Program Evaluation) - Coordination of DOE's international activities.

- OP (Operations Office) - Implementation of HQ policy and directives at DOE sites, issuing orders to specific sites; directing efforts of DOE contractors.

- Contractors - Management and operations of DOE facilities in accordance with $\mathrm{HQ}$ and $\mathrm{OP}$ guidance and orders; national R\&D laboratories.

(contd next page) 


\section{ORGANIZATIONS (contd)}

- DOI (Department of the Interior)

- USGS (U.S. Geological Survey) - Laboratory and field geologic investigations.

- DOT (Department of Transportation) - Development, issuance, and enforcement of safety standards governing aspects of hazardous materials transport, including radioactive materials.

- EPA (Environmental Protection Agency) - Eslablishment and enforcement of general standards for protection of the environment.

- NRC (Nuclear Regulatory Commission) - Issuance of regulations, licenses, and enforcement for commercial nuclear activities and disposal of spent fuel and HLW, in compliance with general environmental standards issued by the EPA; through agreements with states that so desire, delegation of licensing selected types of nuclear facilities. 
DOE (DEPARTMENT OF ENERGY) PARTLAL ORGANIZATION

\section{Secretary \\ Deputy Secretary \\ Under Secretary}

EM - Office of Environmental Management

RW - Omce of Civilian Radioactive Waste Management - YMPO

PO - Office of International Research and Development Policy, Assistant Secretary for Policy, Planning and Program Evaluation

Other Offices

Operations OfTices

- AL - Albuquerque

- LANL - MOUND - RFP - SNL - WIPP

- doo - Chícago

-ANL - BNL - BCO

- ID - Idaho

- INEL - WINCO - WVNS

- NV - Nevada

- dR - Oak Ridge

-ORNL

- dAK - Oalinand Operatlons Oflice

- ETEC - LLNL

- RL - Rlchland

- PNL - WHC

- SR - Savannah River

|WSRC 
PNL-9450-1

UNITED STATES

NRC (NUCLEAR REGULATORY COMMISSION) PARTIAL ORGANIZATION

Chairman

Commlssioners

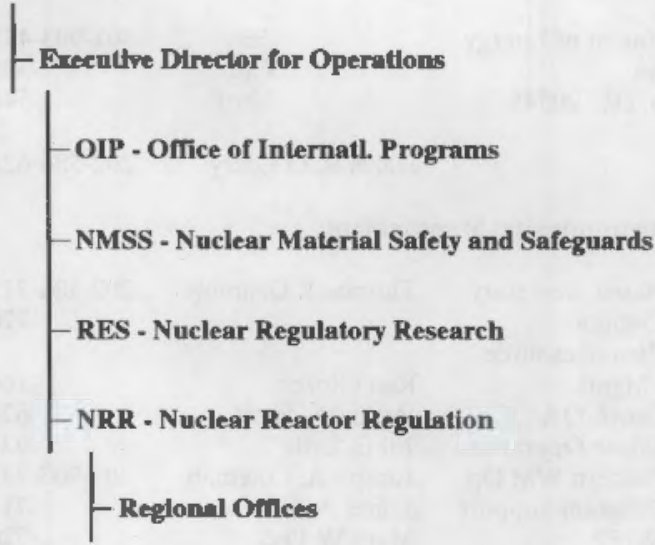

- Region I (Philadelphia)

- Region II (Atlanta)

- Region III (Chicago)

- Region IV (Dallas) 


\section{DOE-Headquarters}

U.S. Department of Energy

Forrestal Building

Washington, DC 20585

U.S. Department of Energy

Germantown

Washington, DC 20545
Tel:

Fax:

Verif:

Tel:

Fax:

Verif:

Hazel R. O'Leary

202-586-6210

Office of Environmental Management

EM-1 Assist. Secretary Thomas P. Grumbly Deputy

202-586-7710

$-7709$

EM-10 Plan./Resource Mgmt.

Ken Glozer

EM-20 Envir. QA/QC

Randal S. Scott

$-1665$

EM-30 Waste Operations

Jill E. Lytle

$-8754$

$-0370$

EM-32 Eastern WM Op.

Joseph A. Coleman

EM-33

Program Support

James A. Turi

Mark W. Frei

EM-34

WIPP

Lawrence H. Harmon

EM-36

Western WM Op.

James Antizzo (A)

EM-40

Hanford WM Op.

John Bavblitz (A)

James J. Fiore

EM-42

Envir. Restoration

Eastern Area

William Wisenbaker

EM-43 Program Support

Sally M. Robison

EM-45

Northwestem Area

Ralph G. Lightner

EM-50

Southwestern Area

Clyde W. Frank

EM-52 Institutional/

Tech. Integration

EM-523

Intnl. Tech. Exch.

Susan M. Prestwich

301-903-7410

$-7147$

$-7201$

$-7105$

$-7170$

202-586-6331

301-903-8141

$-8105$

$-8161$

$-8180$

202-586-6382

$-7924$

$-7640$

EM-54 Research/

Development

Dave W. Geiser

$-7911$

Steven C.T. Lien

$-8490$

EM-55 Dem. Test./Eval. William C. Schutte

EM-60

Facility Transition

Hills W. Bixby

202-586-5151

(contd next page) 


\section{DOE-HQ (contd)}

Office of Civllian Radioactive Waste Management

\begin{tabular}{|c|c|c|c|}
\hline RW-1 & Director & Daniel H. Dreyfus & $202-586-6842$ \\
\hline RW-2 & Dep. Director & Lake Barrett & -6850 \\
\hline RW-3 & Quality Assurance & Donald G. Horton & -8858 \\
\hline RW-4 & Strategic Plng/Intnl. & Jerome D. Saltzman (A & -1252 \\
\hline RW-5 & External Relations & Jerome D. Saltzman & -2277 \\
\hline RW-10 & Prog/Resource Mgmt & Samuel Rousso & -9116 \\
\hline \multirow[t]{2}{*}{ RW-20 } & Geol. Disposal & & \\
\hline & YMPO & $\begin{array}{l}\text { Robert M. Nelson } \\
\text { (As noted below) }\end{array}$ & $702-794-7920$ \\
\hline RW-30 & Systems/Compliance & Dwight E. Shelor & $202-586-9896$ \\
\hline \multirow[t]{11}{*}{ RW-40 } & Storage/Transport & Ronald A. Milner & -9694 \\
\hline & \multicolumn{3}{|c|}{ Yucca Mountain Site Characterization } \\
\hline & Project Omice (YMPC & o) $\quad$ Tel: & $702-794-7900$ \\
\hline & Phase 2, Suite 200 & Fax: & -7907 \\
\hline & $\begin{array}{l}101 \text { Convention Ctr. D } \\
\text { Las Vegas, NV } 89109\end{array}$ & Verif: & -7919 \\
\hline & Manager & Robert M. Nelson (A) & $702-794-5170$ \\
\hline & Dep. Proj. Mgr. & J. Russell Dyer & -798 \\
\hline & Scientific Programs & Susan B. Jones & -7613 \\
\hline & Eng./Field Operations & William S. Simecka & -7933 \\
\hline & Env., Health/Safety & Wendy R. Dixon & -7947 \\
\hline & Suitability/Licensing & Steve J. Brocoum & $202-586-5355$ \\
\hline
\end{tabular}

Omce of Policy, Planning and Program Evaluation

PO-1 Assistant Secretary Susan F. Tierney 202-586-4640

PO-7 D.A.S. Intl. Affairs Richard H. Williamson -5493

PO-70 Intnl. R\&D Policy Harold Jaffe $\quad \mathbf{6 7 7 0}$ 


\section{DOE OPERATIONS OFFICES}

\section{ALBUQUERQUE OPERATIONS OFFICE (AL)}

U.S. Department of Energy

Albuquerque Operations Office

P.O. Box 5400

Albuquerque, NM 87115

Manager

Environment/Spec. Proj.

Uranium Mill Tailings
Tel:

Fax:

Verif:

Bruce G. Twining

Ronald D. Hanson

Albert Chemoff
$505-845-4154$ $-6058$

$-6319$

DOE Rocky Flats Office (Denver Site)

Rocky Flats Plant

P.O. Box 464

Golden, CO 80402-0464

Tel:

Fax:

Verif:

845-6049

$-6210$

$-6134$

Manager

Dep. Manager

Dir., Env. Restoration

Robert M. Nelson, Jr. $\quad$ 966-2025

David P. Simonson $\quad-2025$

Richard Schassburger (A) $\quad-4888$

\section{WIPP}

Waste Isolation Pilot Plant

P.O. Bax 2078

Carlsbad, NM 88221-3090

Tel:

Fax:

Verif:

303-966-7000

$-4092$

$-2719$
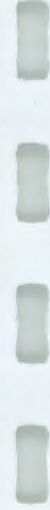

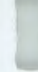

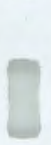




\section{CHICAGO OPERATIONS OFFICE (COO)}

U.S. Department of Energy

Chicago Operations Office

9800 South Cass Avenue

Argonne, IL 60439

Manager

WM/Tech. Devel.

Envir. Restoration

Technical Support

Tel:

Fax:

708-252-2001

Verif:

$-2343$

$-2209$

Cherri J. Langenfeld

252-2110

Joel C. Haugen

$-2093$

Jay Hunze

$-2428$

Antanas Bindokas

$-2692$

\section{IDAHO OPERATIONS OFFICE (ID)}

U.S. Department of Energy

Idaho Operations Office

850 Energy Drive

Idaho Falls, ID 83401-1563

Manager

Deputy Manager

Assistant Manager

Program Execution

SF Program

R\&D

Environmental Restoration

Waste Management

W. Valley Proj. (NY Site)

Process Technology
Tel:

Fax:

Verif:

John M. Wilcynski

Ed L. Wilmot

Thomas F. Burns, Jr.

Robert M. Stallman

Michael J. Bonkoski

Neil S. Burrell

Lisa A. Green

Joel T. Case

Thomas J. Rowland

W. Stephen Ketola
208-526-0111

$-1184$

(recipient)

526-1322

$-1324$

$-7653$

$-1995$

$-1412$

$-1984$

$-0417$

$-6795$

716-942-4313

$716-942-4314$

\section{NEVADA OPERATIONS OFFICE (NV)}

U.S. Department of Energy

Nevada Operations Office

P.O. Box 98518

Las Vegas, NV 89193-8518

Tel:

Fax:

702-295-1212

Verif:

$-1371$

$-1369$

Manager

Nick C. Aquilina

295-3211

Environmental Protection

Don Elle 


\section{OAK RIDGE OPERATIONS OFFICE (OR)}

U.S. Department of Energy

Oak Ridge Operations Office

P.O. Box 2001

Oak Ridge, TN 37831

Manager

Dir., Energy Programs

Dep. AM Energy R\&D

Laboratory Operations

Fusion/Nuclear Tech.

Waste Oper. Branch

Dir., WM/Tech. Dev.

Tel:

Fax:

Verif:

Joe La Grone

Thomas Jelinek

Ronald Hultgren

Connor Matthews

Martha J. Kass

Doug Underwood

Larrv Radcliffe
$615-576-5454$

$-1063$

$-1058$

$576-4444$

$-0710$

$-4523$

$-1373$

$-0717$

$-5973$

$-9212$

\section{OAKLAND OPERATION OFFICE (OAK)}

U.S. Department of Energy Oakland Operations Office 1301 Clay Street

Oakland, CA 94612

Manager, WM

Envir. Safety/Support .

Environmental Radiation

Rad. Safety
Tel:

Fax:

Verif:

Donald Pearman

Daniel Nakahara

Edward Ballard

Ralph Kopenhaver
$510-637-1640$

$-2001$

$-1585$

637-1800

$-1840$

$-1594$

$-1597$ 


\section{RICHLAND (HANFORD) OPERATIONS OFFICE (RL)}

U.S. Department of Energy

Richland Operations Office

825 Jadwin Avenue

P.O. Fox 550

Richland, WA 99352

Tel:

509-376-7411

Fax:

$-6540$

?

Manager

Deputy Manager

Verif:

$-7317$

Performance Assessment

John D. Wagoner

509-376-7395

Ron A. Izatt

$-4216$

R. Pierre Saget

$-2611$

Env. Assurance/Permits/Policy Steve H. Wisness (A)

$-6798$

AM, Technical Support

Planning/Integration

John J. Keating

$-7387$

Quality/Safety/Health

William A. Rutherford

$2-2500$

Laboratory Management

Technology Development

AM, Envir. Restoration

Richard A. Holten

$-7461$

Joseph J. Sutey

$2-4005$

TBD

Environmental Restoration

James D. Bauer

$-6628$

Roger D. Freeberg

$-7277$

Environmental Remediation

Project Management

AM, Waste Management

Nuclear Materials

Transition

Julie K Erickson

$-3603$

Larry C. Williams

$-4131$

John R. Hunter

James L. Daily (A)

$-7434$

$-7721$

$-7471$

Waste Management

James E. Mecca

$-1366$

June M. Hennig

$-5494$

Solid Waste/Transportation

Rudolph F. Guercia

Thomas R. Sheridan (A)

$-7591$

\section{SAVANNAH RIVER OPERATIONS OFFICE (SR)}

U.S. Department of Energy

Savannah River Operations Office

P.O. Box A

Tel:

Fax:

803-725-6211

Aiken, SC 29801

$-2033$

$-1259$

Manager

Mario Fiori

AM, EM/Solid Waste

Tom F. Heenan

$725-2277$

$-8074$

Deputy Assistant Manager

Dir., Solid Waste

Dir., EM

Jessie M. Roberson

$-8571$

Michael G. O'Rear

$-5541$

TBD

$-3966$

Director, D\&D

Richard D. Lynch

$-8924$

PM/TPO, OTD

James A. Wright

$557-0558$ 


\section{DOE CONTRACTORS}

ANL

Argonne National Laboratory 9700 South Cass Avenue Argonne, IL 60439

Director

Waste Mgmt.

Separations Science/Tech.

Pyroprocessing

Special Projects

ANL-West (ID)
Tel:

Fax:

708-252-2000

Verif:

Alan Schriesheim

James E. Helt

George Vandegrift

James J. Laidler

Charles E. Klotz

Charles E. Till
$-2343$

$-2206$

$-2209$

252-3872

$-7335$

$-4513$

$-4479$

$-6385$

208-533-7000

Fuel Cycle and Waste Management Activities: Remedial action for formerly used AEC sites (FUSRAP) and for surplus facilities management program (SFMP); D\&D of ANL-East (Argonne, IL) contaminated facilities; mixed waste treatment and disposal, groundwater treatment; LLW/TRU waste technology; TRUEX process development; pyrometallurgical and pyrochemical fuel reprocessing, electrorefining; Environmental Restoration and Waste Management; applied R\&D program support for EM; SARP review; Civilian Radioactive Waste, socioeconomic impact assessment, transportation planning, spent fuel and waste glass performance, interaction of waste package with repository environment, instrumentation development.

Major Facilities:

ANL-East (Argonne, IL): High-Level Hot-Cell Facilities; Large Gamma Radiation Facility; Alpha-Gamma Hot-Cell Facility (AGHCF).

ANL-West (Idaho Falls, ID): Experimental Breeder Reactor No. 2 (EBR-II); Zero Power Plutonium Reactor (ZPPR); Transient Reactor Test Facility (TREAT); Hot Fuel Examination Facility (HFEF); Radioactive Scrap and Waste Facility; Sodium Process Demonstration (SPD) Facility; Radioactive Liquid Waste Treatment Facility (RLWTF); Fuel Cycle Facility (FCF). 


\section{$\underline{\text { BCO }}$}

Battelle - Columbus Operations

Tel:

614-424-6424

$505 \mathrm{King}$ Avenue

Columbus, $\mathrm{OH} 43201$

Fax:

$-5601$

Verif: numbers below

Environmental Systems and Technology Division

VP/General Manager

W.J. Madia

424-7359

Technical Operations

G.J. Kovacs

$-7937$

Nuclear Safety

Environmental Restoration

R.S. Denning

$-7412$

Systems Management

J. Means

C.P. Nunn

Process Safety/Risk Mgmt. D\&D

W.W. Simmons

K.C. Brog

Fuel Cycle and Waste Management Activities: Site survey/ characterization; waste packaging; disposal technology; trans. portation; performance assessment; safety analysis reports; environmental/socioeconomic assessments; decontamination and decommissioning; systems integration; quality assurance; licensing; nuclear engineering/technology; policy support; institutional interactions; communications and outreach; safety and training; regulatory compliance.

Hazardous Chemical and Mixed Wasle Activities: Transportation; risk assessment; modeling; regulation; waste management; policy support; regulatory compliance. 


\section{BNL}

Brookhaven National Laboratory

Associated Universities, Inc.

Uplon, NY 11973
Tel:

Fax:

Verif:
$516-282-2123$

$-3000$

$-2547$

282-2772

$-3045$
N. P. Samios

Peter Colombo
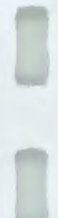

Fuel Cycle and Waste Management Activities: Waste stabilization/ solidification processes; mixed waste form performance criteria and testing protocol; subterranean waste confinement barriers; performance and risk assessment; geochemistry of contaminated soils and sediments; materials characterization and evaluation.

Major Facilities: Hot and Cold Development Laboratories: CO-60 Gamma Irradiation Facility; National Synchrotron Light Source; Alternating Gradient Synchrotron; High Flux Beam Reactor; Scanning Transmission Electron Microscope.

\section{ETEC}

Energy Technology Engineering Center

Rockwell International

Tel:

818-586-5326

P.O. Box 7930

Fax:

$-5118$

Canoga Park, CA 91309-7930

Verif:

$-5326$

General Manager

D. Clark Gibbs

$586-5326$

Bruce B. Gillies

$-5301$

G. Subbaraman

Tech. PM

Fuel Cycle and Waste Management Activities: Decontamination and decommissioning (D\&D) of structures (test reactors and hot cells) and open sites; D\&D technology development; liquid metal (sodium and $\mathrm{NaK}$ ) waste destruction; characterization and final remediation surveys; pathway analysis models; statistical treatment of survey data for regulatory compliance; statistical/computational code for estimating and displaying spatial contaminant distribution.

Major Facilities: Radioactive Materials Disposal Facility (RMDF). 


\section{INEL}

Idaho National Engineering Laboratory

EG\&G Idaho, Inc.

Tel:

208-526-0111

P.O. Box 1625

Fax: $-8339$

Idaho Falls, ID 83415

Verif:

(recipient)

General Manager

Earl Fray

$526-9671$

Environmental

Restoration/WM

Power Reactor Program

Waste Technology

Development

Jim McAnally

$-1014$

Jim Okeson

Ken Merrill

$-0797$

Fuel Cycle and Waste Management Activities: National LLW technology; D\&D operation of SWEPP for TRU waste; LLW disposal operation; cask systems development; SF cask transport and testing; treatment and disposal of hazardous and mixed wastes; pollution prevention; WIPP support activities; remediation and treatment of buried TRU waste.

Major Facilities: Radioactive Waste Management Complex (RWMC); Waste Experimental Reduction Facility (WERF); Stored Waste Examination Pilot Plant (SWEPP) for TRU wastes; Test Area North Spent Fuel Storage Area (TAN); Advanced Test Reactor (ATR); Transuranic Storage Area. 
LANL

Los Alamos National Laboratory

University of California

P.O. Box 1663

Tel:

Fax:

505-667-5061

Los Alamos, NM 87545

Verif:

Director

Siegfried S. Hecker

667-5101

Director, Envir. Mgmt.

Thomas Baca

$-2211$

PM, EM

Jong Jansen

665-6295

PM, WM

Group Leader, WM

TBD

PM, Nuc. Tech./

Anthony Drypoleer

667-7391

Reconfiguration

Paul Cunningham

$-9807$

Fuel Cycle and Waste Management Activities: Fundamental studies of waste materials (BES); migration from LLW (BES); D\&D of various site facilities; HLW disposal site characterization (RW).

Major Facllities: Waste Disposal Field Experimental Facility; Controlled Air Incinerator Facility; Size Reduction Facility; TRU Waste Assay Systems; Advanced Testing Line for Actinide Separations (ATLAS). 


\section{LLNL}

Lawrence Livermore National

Laboratory

University of California

P.O. Box 808

Livermore, CA 94550

Tel:

$510-422-1100$

Fax:

423-1997

Verif:

423-0672

Director

Dir., YMSCP

C. Bruce Taner (A)

422-4169

Energy Director

Wiliis L. Clarke

423-4571

David E. Baldwin

$-1415$

Fuel Cycle and Waste Management Activities: SNM disposition; waste form characterization; near-field environment characterization (geochemistry, geohydrology, geomechanies); engineered barrier system (EBS) concept development; scientific bases for waste package design; EBS materials selection and characterization; EBS performance analysis; international programs (spent fuel, introduced materials, natural analogs).

Major Facility: Large Block Test at Fran Ridge-Yucca Mountain, Nevada.

\section{MOUND}

EG\&G Mound Applied Technologies P.O. Box 3000

Miamisburg, $\mathrm{OH} 45343-3000$

Tel:

Fax:

Verif:

513-865-4020

$-3742$

$-3575$

General Manager

Jack L. Clark

865-3576

Tritium Technology

Eric T. Kirk

$-4842$

D\&D

Ralph R. Jaeger

$-3275$

Waste Management

James F. Griffin

$-3097$

(contd next page) 


\section{MOUND (contd)}

Fuel Cyele and Waste Management Activities: Mixed waste treatment with glass melter, TRU waste technology/record systems; tritium recovery from scrap; D\&D of ${ }^{228} \mathrm{Pu}$ facilities.

Major Facilities: Glass Melter, Liquid Radioactive Waste Treatment Facility; Combined Electrolysis Catalytic Exchange System (CECE); Thitium Effluent Recovery System (ERS); Hydrogen Isotope (Cryogenic Distillation) Separation System (HISS); Tritium Aqueous Waste Recovery System (TAWRS).

\section{ORNL}

Oak Ridge National Laboratory

Martin Marietta Energy

Systems, Inc.

Tel:

615-576-5454

P.O. Box 2008

Oak Ridge, TN 37831

Fax:

Verif:

6-2900

4-7456

$-7065$

$-6461$

6-1092

Director

Alvin Trivelpiece

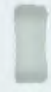

Dir., Robotics/Process Systems

Dir., OCRWM Programs

Ronald Pope

Tony Malinauskas

Waste Management Activities: Operate waste management facilities, including disposal; develop LLW \& TRU waste treatment technology, including assay and package certification; hazardous waste remedial actions; waste operations control center; UMTRA radiological survey; environmental restoration and facilities upgrade; waste management R\&D.

Major Facillties: LLW disposal/storage facilities; Waste Examination Assay Facility (WEAF); Tower Shielding Facility (fuel/waste cask drop tests); TRU storage/certification facilities; liquid LLW processing/storage; waste processing/disposal; Tumulus LLW Disposal Facility; Non-Radiological Wastewater Treatment Plant; hazardous waste storage and packaging facility.

(contd next page) 


\section{ORNL (contd)}

Fuel Cycle and Reprocessing Activities: Develop reprocessing, remote systems, safeguards technologies, and facilities design optimizations.

Major Facilities: Integrated Equipment Test Facility including Fuel Element Disassembly and Shearing Systems, Continuous Rotary Dissolver, Chemical Rack Systems, Advanced Integrated Maintenance System, and Environmental Test Chamber.

\section{PNL}

Pacific Northwest Laboratory Battelle, Pacific Northwest

Laboratories

902 Battelle Boulevard

P.O. Box 999

Tel:

Fax:

Verif:

509-375-2121

Richland, WA 99352

Director

Engineering Technology

Waste Treatment

Technology

Waste Process

Engineering

Waste Systems

Intnl. Program Support

Matls./Chem. Sciences

Earth/Envir. Science

Industrial Programs

EM Programs

Environmental Operations

Envir. Tech. R\&D

William R. Wiley

376-3876

(recipient)

Bill D. Shipp

5-2201

5-2921

Richard A. Brouns

2-2219

Charles R. Allen

Gary W. McNair

6-1712

Sue J. Mitchell

5-6537

Walter W. Laity

5-6479

5-2780

Michael J. Graham

6-8314

J. Adrian Roberts

5-2614

Joseph D. Spencer

$5-4331$

Mark S. Hanson

5-6812

Steve C. Slate

5-3903

Tank Waste Remediation

Waiter J. Apley

2-4664

(contd next page) 


\section{$\underline{\text { PNL (contd) }}$}

Fuel Cycle and Waste Management Activities: Waste storage and transportation; LLW, HLW, mixed, and TRU waste characterization and treatment; waste tank remediation; international program support in waste management/environmental remediation; radioisotope separation and use; damaged spent fuel stabilization; in-situ treatment and barriers for contaminated sites; disposal performance assessment; reactor safety and advanced design; integration of geologic disposal systems; management of surplus plutonium; D\&D planning and technologies; assessment of past radioactive releases; environmental information systems; public involvement processes; risk management tools and health effects; analysis of environmental regulations; and integrated environmental planning and management approaches.

Major Facillties: Hot and cold analysis laboratories and development laboratories. Hot cells for pilot scale programs. Facilities for large-scale demonstrations and major basic science research.

\section{RFP}

Rocky Flats Plant

EG\&G Rocky Flats, Inc.

Tel:

303-966-7000

P.O. Box 464

Fax:

Golden, CO 80402-0464

Verif:

General Manager

H. P. Mann

966-4361

Waste Operations

R. V. Morgan

$-6012$

Waste Minimization

A. L. Schubert

$-6595$

Technology Development

T. L. Rising

$-4725$

Fuel Cycle and Waste Management Activities: Defense TRU waste technology; LLW technology development; waste treatment facilities operations, TRU/LLW minimization technology.

Major Facilities: Solid Waste Reduction Facility; TRU Waste Supercompaction; TRU Waste Assay; Liquid Waste Treatment and Fixation Facilities; Microwave Melting of Liquid Waste Treatment Sludges. 
SNL

Sandia National Laboratory P.O. Box 5800

Albuquerque, NM 87185-5800

Tel:

Fax:

Verif:

505-844-5678

$-1068$

$-8917$

President

Al Narath

4-7261

VP, Energy \& Environment

Dan Hartley

$5-9488$

Energy \& Environ.

Envir. \& Transportation

Nuclear Waste Mgmt.

Nuclear Energy Technology

Reactor Engineering

Thomas O. Hunter

4-3763

Joan Woodard

5-9917

Dori Ellis

$848-0780$

Nestor Ortiz

$4-0577$

Jim Rice

5-7301

Fuel Cycle and Waste Management Activities: ER/WM technology development, radioactive waste management (Yucca Mountain, WIPP, Greater Confinement Disposal, LLW), waste management strategic planning, reactor safety, new production reactor, reactor engineering technology.

Major Facilities: Research reactors and numerous test facilities. 


\section{SRS}

Westinghouse Savannah River Company (WSRC)

Savannah River, TE

Tel:

803-725-6211

P.O. Box 616

Aiken, SC 29802

Fax: $-1660$

Verif:

VP/General Manager

Norm F. Boyler

$-5331$

Solid Waste/Environ. Rest.

Deputy General Manager

Solid Waste

Environmental Restoration

Transition, D\&D

Program Management

VP/Gen. Manager

HLW Tech. Director, HLW

HL Liquid Waste

DWPF

Gale K. Hovey

952-7193

Hugh F. Daugherty

2-7189

A.M. (Sam) Schwartzman

$557-6305$

Dennis A. Stevenson

$644-6841$

Clay B. Jones

$-5021$

Austin B. Scott

952-7917

Harry D. Harmon

$725-2585$

G. Todd Wright

725-3887

David B. Amerine

$557-1527$

$557-1050$

Fuel Cycle and Waste Management Activities: Operate fuel reprocessing facilities and associated spent fuel storage, HLLW tank storage, and treatment facilities for defense waste; operate LLW shallowland burial grounds; start up and operate DWPF; store mixed waste; site remediation.

Major Facilities (existing and planned): Defense Reprocessing Plants; Canyon Mockup Shop; LLW Incinerator; HLW Tank Farm; Defense Waste Processing Facility (DWPF); Hazardous Waste/ Mixed Waste Processing Facility; Consolidated Incinerator Facility (Hazardous, LLW, and Mixed Waste); Transuranic Waste Facility; LLW Preparation Facility.

(contd next page) 
SRS (contd)

Savannah River Technology Center

Westinghouse Savannah River

Company (WSRC)

Savannah River, TE

Building 773A

Fax:

803-725-1660

Aiken, SC 29808

Verif:

$-2304$

Vice President/Director

Crispin W. Thiessen (A) 725-3422

WM/Environment

Lucien M. Padouchado

TPM, OTD

John L. Steele

Inter. Prog. Coord.

Bruce G. Kitchen

Fuel Cycle and Waste Management Activities: Fuel reprocessing R\&D; HLW storage and solidification R\&D; HLW form development and characterization; HLW packaging R\&D; TRU technology development; LLW technology development; defense HLW technology development; mixed/hazardous waste technology development; groundwater remediation technology development.

Major Facillties: HLW Vitrification Pilot Plant; HLW Tank Mockup; HLW Caves for Process Development; Groundwater Remediation Demonstration; Bioremediation Demonstration; MLLW Vitrification. 
$\underline{\text { TRW }}$

TRW Environmental Safety

Systems Inc.

2650 Park Tower Drive

Tel:

703-204-8500

Vienna, VA 22180

Fax:

$-8580$

President/General Mgr.

Asst. Gen. Mgr. Operations

Roland L. Robertson

Ray W. Godman

204-8600

Asst. Gen. Mgr. Systems

Arthur S. Kubo

$-8605$

Asst. Gen. Mgr. NV

L. Dale Foust

$-8680$

Fax:

Function: Management and Operating Contractor $(\mathrm{M \& O})$ for the DOE Office of Civilian Radioactive Waste Management (OCRWM); supports OCRWM through systems engineering, design, development, and technical direction of the Civilian Radioactive Waste Management System. The OCRWM mission is permanent disposal of the nation's spent nuclear fuel and HLW in a manner that protects the health and safety of the public and the quality of the environment. 
WHC

Westinghouse Hanford Company

P.O. Box 1970

Tel:

Fax:

Richland, WA 99352

Verif:

509-376-7411

$-4668$

$-5777$

President

A. LaMar Trego

376-7803

Dir., President's Office

Ronald E. Lerch

$-5107$

Exec. VP, TWRS

William T. Alumkal

373-3563

VP, Transition Projects

Ronald J. Bliss

$376-6427$

VP, Solid Waste

Dir., Spent Fuel

Henry E. McGuire

376-1400

John C. Fulton

373-5823

Fuel Cycle and Wasle Management Activities: Hanford Site operating contractor; HLW tank storage; $\mathrm{Cs} / \mathrm{Sr}$ recovery and encapsulation; HLW concentration and solidification; liquid LLW treatment and fixation; TRU waste assay; Hanford waste disposal; breeder fuel development and fabrication; spent fuel storage; solid waste disposal operations.

Major Facillties: Plutonium Finishing Plant; Fast Flux Test Facility (shut down); Fuel Development Laboratories; PUREX Plant (shut down 1992); UO3 Plant; B Plant; Tank Farms. 
Westinghouse Idaho Nuclear

Company, Inc.

P.O. Box 4000

Idaho Falls, ID 83415-3201

Tel:

208-526-0111

Fax:

$-7146$

Verif:

(recipient)

President

VP/Director, Applied

Technology

VP/Manager Operations

William C. Moffitt

$526-0998$

Len F. Ermold

$-4628$

Steve M. Halupa

$-9003$

Fuel Cycle and Waste Management Activities: Receive and store spent nuclear fuel from Navy and other DOE sources, including characterization and technology development for final disposal; manage and process liquid and solid LLW and HLW (including necessary technology development) for final disposal.

Major Facllities: Fluorinel and Storage Facility (FAST), New Waste Calcining Facility (NWCF), Liquid Effluent Treatment and Disposal (LET\&D) facility, Remote Analytical Laboratory (RAL), Idaho Research Center (IRC), and Fuel Processing Facility (FPF) - not yet complete.

\section{WVNS}

West Valley Nuclear Services Co., Inc.

P.O. Box 191

Tel:

$716-942-3235$

10282 Rock Springs Road

West Valley, NY 14171-0191

Fax: $\quad-4246$

Verif: $\quad-4750$

President

William G. Poulson

VP/Deputy Project Mgr.

James L. Little

Fuel Cycle and Waste Management Activities: Demonstration of HLW vitrification; supernatant treatment by ion exchange; LLW treatment using cement solidification.

Major Facllities: HLW Vitrification Facility; Integrated Radioactive Treatment System (supernatant processing, evaporation, remote cementation facility, product storage). 


\section{OTHER U.S. ORGANIZATIONS}

\section{EPA}

Environmental Protection Agency $401 \mathrm{M}$ Street S.W.

Washington, DC 20460

International Activities

Assistant Administrator

Multilat. Staff Dir.

Radiation Programs

Director

Criteria Stnds.

Waste Management
Tel:

Fax:

Verif:

202-260-2090

$-7883$

$-2078$

Dr. Alan D. Hecht

Pat Koshel

260-4870

$-0797$

Margo T. Oge

J. William Gunter

Lawrence Weinstock

233-9320

$-9290$

$-9310$

\section{Solld Waste}

Director

Michael H. Shapiro

Dep. Dir., State Programs

Matthew Hale

Function: Establish and enforce standards for protection related to radioactive and hazardous waste.

\section{$\underline{\text { EPRI }}$}

Electric Power Research Institute

3412 Hillview Avenue

P.O. Box 10412

Palo Aito, CA 94303

Tel:

Fax:

Verif:

415-855-2000

President

Richard Balzhiser

VP/Director, Nuc. Power

John J. Taylor

$-2800$

$-2674$

Fuel Performance Stor.Disp.

Low-Level Waste

Rosa Yang

855-2141

Christopher J. Wood

$-2030$

$-2481$

$-2379$

Fuel Cycle

Robert Williams

$-2064$

(contd next page) 


\section{EPRI (contd)}

Fuel Cycle and Waste Management Activities: Spent fuel rod consolidation study; cooperative on-site demonstration of spent fuel storage in metal casks/concrete silos; conceptual designs for LLW disposal sites; demonstration of transportable spent fuel metal storage casks; spent fuel storage and transportation studies; fuel failures, fuel cladding corrosion, high-temperature operation and extended burnup; fuel performance computer models; HLW repository performance assessment.

\section{$\underline{\text { NRC }}$}

U.S. Nuclear Regulatory Commission Washington, DC 20555

Tel:

Fax:

Verif:

301-492-7000

$504-2260$

$-2262$

Chairman

Ivan Selin

Commissioner

Kenneth C. Rogers

504-1759

$-1855$

Commissioner

Forrest J. Remick

$-1820$

Commissioner

E. Gail de Planque

$-1800$

Commissioner

Vacant

Ofice of International Programs (OIP)

Director

Bilat, Cooperation/Asst.

Non-Prolif./Exp./Multilat. Rel. Ronald D. Hauber

Omice of Nuclear Material Safety and Safeguards (NMSS)

Director

Robert M. Bernero

Fuel Cycle Safety/Safeguards

Indust./Medical Nuc. Safety

Waste Management

Robert F. Burnett

Carl J. Paperiello

Malcolm R. Knapp
Carlton R. Stoiber James R. Shea

$504-1780$

$-2336$

$-2344$

$415-7800$

$-7212$

$-7264$

$-7437$

(contd next page) 
$\underline{\text { NRC (contd) }}$

Omce of Nuclear Reactor Regulation (NRR)

Director

Reactor Projects I/II

Reactor Projects III/IV/V

Adv. Reactors/

License Renewal

Operating Reactor Support

Systems Safety/Analysis

Reactor Insp./Licensee

Perform.

Reactor Controls/Human

Factors

Radiation Safety/Safeguards

William Russell

Steven A Varga

301-504-1270

$-1403$

Jack W. Roe

$-1354$

Dennis M. Crutchfield

$-1199$

Brian K Grimes

$-1163$

Vacant

$-2884$

Charles E. Rossi

$-2903$

Bruce A. Boger

$-1004$

Frank J. Congel

$-1088$

Office of Nuclear Regulatory Research (RES)

Director

Eric S. Beckjord

Engineering

Safety Issues Resolution

Lawrence C. Shao

492-3700

Warren Minners

$-3800$

Brian Sheron

$-3900$

Systems Research

Regulatory Applications

Bill M. Morris

Function: Issue regulations and licenses and enforce thern for commercial nuclear activities and disposal of spent fuel and HLW, in compliance with general environmental standards issued by the EPA; carry out $\mathrm{R} \& \mathrm{D}$ to support regulatory function.

\section{Regional Orices}

$\begin{array}{ll}\text { Philadelphia } & - \text { Region I } \\ \text { Atlanta } & \text { - Region II } \\ \text { Chicago } & - \text { Region III } \\ \text { Dallas } & \text { - Region IV }\end{array}$

Thomas T. Martin

215-337-5299

Stewart D. Ebneter

404-331-5500

John B. Martin

708-829-9657

Robert D. Martin

$817-860-8225$ 


\section{NWTRB}

U.S. Nuclear Waste Technical

Review Board

1100 Wilson Boulevard, Suite 910

Arlington, VA 22209

Tel:

703-235-4473

Fax:

$-4495$

Verif:

$-4473$

Chairman

Executive Director

Dir., External Affairs

John E. Cantlon

William D. Barnard

Paula N. Alford

Function: Established by Congress in the Nuclear Waste Policy Amendments Act of 1987 to provide independent review of DOE's technical and scientific program for the disposal of commercial spent nuclear fuel and defense HLW. At full complement, eleven members serve on the Board; all are appointed by the President.

\section{ONWN}

Office of the Nuclear

Waste Negotiator

1823 Jefferson Place, N.W.

Washington, DC 20036

$\begin{array}{lr}\text { Tel: } & 202-634-6244 \\ \text { Fax: } & -6251 \\ \text { Verif: } & -6244\end{array}$

Office of the Nuclear Waste Negotiator

3050 North Lakeharbor Lane

Suite 100

Boise, Idaho 83703

Tel:

Fax:

Verif:

208-334-9876

$-9880$

$-9876$

Negotiator

Deputy Negotiator

Science Advisor
Richard H. Stallings

Robert Mussler

Dr. Robert Limatainen

Function: Established by the Nuclear Waste Policy Amendments Act of 1987 as an independent executive agency of the federal government to identify a state or Indian tribe willing to host an MRS facility or permanent repository for HLW. Authorized to negotiate with interested potential hosts to determine the terns and conditions nnder which they would agree to serve as host to put such agreement in the form of legislation and submit it to Congress for enactment into law. 


\section{USGS}

U.S. Geological Survey 410 National Center 12201 Sunrise Valley Drive Reston, VA 22092

Tel:

703-648-4000

Fax:

$-5295$

Verif:

$-5235$

Director

Gordon P. Eaton

648-7411

Asst. Dir./Eng. Geology

Nuclear Waste Hydrology

HLW

LLW

James F. Devine

$-4423$

Newell J. Trask

$-5719$

Peter R. Stevens

$-5721$

Toxic Waste

Gail Mallard

$-6872$

YMP TPO (Denver Office) Larry R. Hayes

303-776-0516

Fuel Cycle and Waste Management Activities: Basic/applied research on hydrogeologic processes relevant to radioactive and toxic waste disposal; geologic/hydrologic investigations to determine suitability of potential HLW repository site at Yucca Mountain; site investigations/research at DOE and DOD installations and EPA Superfund sites; consultant for EPA, DOE, DOD, Dept. of Agriculture (DOA), Bureaus of Land Management (BLM), Mines (BOM), and Reclamation (BOR), and state agencies. 


\section{INTERNATIONAL AGENCIES}




\section{CEC}

Commission of the European

Communities

200 Rue de la Loi

1049 Brussels, Belgium

Tel: $\quad 32-2-299-1111$

Fax: $\quad 32-2-295-0138$ or $-0139,-0140$

Commissioner for Science,

Research and Development, Joint Research Centres

Director-General, Science/R\&D

Director-General, JRCs, Deputy

Director-General, Science/R\&D

Director, Energy R\&TD

Division, Fuel Cycle \& Safety

R\&D Program RWM

Safety Studies

Waste Form R\&D

Disposal Studies

URLs

R\&D Program D\&D Nuc. Installations

Antonio Ruberti

Paolo Fasella

Hendrik Tent

Herber J. Allgeier

32-2-295-4055

Werner Balz (A)

Nicolo Cadelili

32-2-295-4055

Nicolo Cadelli

Michel Hugon

Henning von Maravic

Bertus Haijtink

Rainer Simou

32-2-295-6623

Division, Radiological Protection Jaak Sinnaeve

Director-General, Environment, Nuclear

Safety \& Civil Protection

Director, Nuclear Safety, Industry/

Environment, Civil Protection

TBD

Edward Bennett

Division, Radiation Protection

Hans Eriskat

32-2-295-2251

Division, Envir. Monitoring

Director, Euratom Safeguards

Dir. Gen., Euratom Supply Agency

George Fraser

Wilhelm Gmelin

32-72-32-211

Michael Goppel

32-2-295-7894

(contd next page) 
CEC (contd)

MEMBER STATES - EUROPEAN COMMUNITY (EC)

$\begin{array}{lll}\text { Belgium } & \text { Greece } & \text { Netherlands } \\ \text { Denmark } & \text { Italy } & \text { Portugal } \\ \text { France } & \text { Ireland } & \text { Spain } \\ \text { Germany } & \text { Luxembourg } & \text { United Kingdom }\end{array}$

\section{FUNCTION}

Executive body for the European Communities (combined Euratom, Coal and Steel, Common Market).

\section{FUEL CYCLE PROGRAM ADMINISTRATION}

\section{R\&D Programs}

- Direct action--fully funded by CEC (through tax on Member States); conducted by Joint Research Centre establishments at Ispra (Italy) and Karlsruhe (Germany).

- Shared-cost action--coordinated and partly (50\%) funded by CEC HQ under cost-sharing contracts; conducted by research centers, universities, and industries in the Member States:

- Decommissioning of Nuclear Installations

- Radioactive Waste Management and Disposal

- Remote Handling in Hazardons Nuclear Environment Robotics

\section{Cooperation Programs}

Participation/support in joint project with various nations and/or other international organizations.

DOE/CEC Agreement for Waste Management Technology Exchange

Term: $\quad 10-6-82$ to $10-6-92$

Scope: Characterization of waste forms; disposal in geologic formations; emphasis on R\&D. 


\section{CEC-JRC: ISPRA}

CEC Joint Research Center Ispra Establishment

21020 Ispra (Varese)

Italy

Tel: $\quad 39-332-78-9111$

Fax: $\quad 39-332-78-9045$

Location: Northern Italy; may be reached by air travel to Milan, ground transport to Ispra, about $50 \mathrm{~km}$.
Safety Technology
H. Holtbecker
Nuclear Fuel Cycle R\&D
H. Dworschak

Waste Management R\&D: R\&D in treatment and storage of radioactive wastes; volume reduction and conditioning TRU wastes; actinide separation; nnclide assay in wastes.

\section{CEC-JRC: KARLSRUHE}

Karlsruhe Joint Research Centre

(European Institute for

Transuranium Elements)

Postfach 2266

76125 Karlsruhe

Federal Republic of Germany

Tel; $\quad$ 49-72A7-841

Fax: $\quad 49-7247-95-1590$

Director

Dr. Jacques van Geel

Location: On the site of the German Nuclear Research Center KfK in Linkenheim, near Karlsruhe.

Function: Basic research in the transuranium elements, especially plutonium; resctor fuels development; R\&D on actinide partitioning and transmutation.

Fuel Cycle R\&D: Plutonium conversion and plutonium fuels.

Waste Management R\&D: Characterization of vitreous HLW forms and $\mathrm{SF}$ when considered as waste.

Safeguards R\&D: Fissile material solution analyses. 


\section{IAEA}

International Atomic Energy

Agency

Wagramer Strasse 5

Tel: $\quad 43-1-2360-00$

P.O. Box 100

1400 Vienna, Austria

Tel: $\quad 43-1-2360$-ext.

Fax: 43-1-2345-64

Director-General

Dep. Dir.-Gen. Nuc. Energy/Safety

Dir. Nuc. Fuel Cycle/Waste Mgmt.

Head, Waste Management

Waste Mgmt./U.S. Staff

RADWASS Program

Head, Nuc. Mtls./Fuel Cycle Tech.

Dep. Dir.-Gen. Safeguards

Dep. Dir.-Gen. Tech. Cooperation

Dep. Dir.-Gen. Research/Isotopes

Dep. Dir.-Gen. Administration

Hans Blix

$-1111$

Boris Semenov $\quad-2600$

Robert Baschwitz $\quad-2650$

Donald E. Saire $\quad-2674$

Candace Y. Chan $\quad-2607$

John R. Wiley $\quad-6097$

Emst Warnecke $\quad-2676$

Norubu Oi $\quad-2766$

Bruno Pellaud $\quad-1800$

Jijui Qian $\quad-2300$

Sueo Machi $\quad-1600$

David B. Waller $\quad-\mathbf{1 0 2 0}$

\section{MEMBER STATES}

120 nations (U.N. members, including the U.S.).

\section{FUNCTION}

Autonomous intergovernmental organization established in 1957 in accordance with a decision of the General Assembly of the United Nations; authorized to foster research and development in the peaceful uses of nuclear energy and exchange of scientific and technical information; establish and administer safeguards against the diversion to military purposes of nuclear materials intended for use in civil nuclear programs; and to establish or administer health and safety standards. 


\section{IAEA (contd)}

\section{WASTE MANAGEMENT ACTIVITIES}

- Collect, prepare, review, and disseminate technical and scientific information on

- planning of waste management systems and programs

- handling, treatment, storage, and conditioning of waste, including uranium mill tailings

- disposal of waste

- assessment of the radiological and environmental consequences of waste management

- decontamination and decommissioning of nuclear facilities

- environmental restoration

- Develop and promote international consensus documents (Safety Fundamentals, Standards, Guidelines, and Practices) in all areas of radioactive waste management - implemented through the Radioactive Waste Safety Standards Programs: RADWASS

- Provide direct assistance to Member States through the

- Waste Management Advisory Program - WAMAP - focused on developing nuclear programs

- Waste Management Assessment and Technical Review Program WATRP - an international peer review service for developed programs

- Serve as scientific and technical body for international conventions (e.g., London Convention) and multinational projects

(i.e., International Arctic Seas Assessment Program - LASAP

- Assist in and facilitate international/multinational projects (e.g., UNDP and UNEP)

(contd next page) 


\section{IAEA (contd)}

- Promote and sponsor research work and development of data and technologies through Technical Assistance Projects and Coordinaled Research Projects.

U.S. Mission to IAEA (UNVIE)

Obersteinergasse 11

1190 Vienna, Austria

Tel: $\quad 43-222-36-3152$

Fax: 43-1-364-1585

Nuclear Policy

Michael J. Lawrence

Nuclear Technology

Bruce Cooper

Safeguards

Marvin Peterson

Science Attaché

Lisa Hilliard 


\section{PARTIAL IAEA ORGANIZATION}

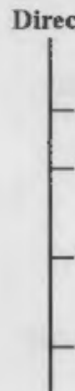

- Hans Blix

- Bruno Pellaud

- Jihui Qian

- Sueo Machi

- Boris Semenov Energy/Safety

- Dir., Nuclear Fuel Cycle/WM \begin{tabular}{|l} 
Waste Management \\
\hline - Predisposal \\
- Disposal \\
- D\&D \\
- Rad./Env. Effects \\
- RADWASS/WATRP \\
Nuc. Mtls./FC Tech. \\
- Raw Materials \\
- Fuel Tech/Perform. \\
- SF Management
\end{tabular}

- Dir., Nuclear Safety

- Dir., Nuclear Power

- Dir., Sci/Tech. Information
- Robert Baschwitz

- Donald E. Saire

- Vladimir Tsyplenkov

- Arnold Bonne

- Mike Laraia

- Gordon Linsley

- Ernst Warnecke

- Noboru Oi

- Mohamad Tachid

- Georgi Sukhanov

- Feree Taknts

- Morrís Rosen

- Poong-Eil Juhn

- Joyce Amenta 


\section{$\underline{\text { ICRP }}$}

International Commission on Radiological Protection

Clifton Avenue

Sutton, Surrey SM2 5PU

United Kingdom

Tel: $\quad 44-81-642-4680$

Fax:

Chairman, Main Commission

Scientific Secretary

Prof. Roger Clarke

Dr. Hyton Sinith

\section{FUNCTION}

Provide principles of radiation protection as a basis for each country to use in establishing technical codes of practice.

\section{OECD}

Organisation for Economic

Co-Operation and Development

2, Rue Andre-Pascal

F-75775 Paris Cedex 16, France

Secretary General

Dep. Secretary General

Dep. Secretary General

Dep. Secretary General

Tel: $\quad 33-1-45-24-82-00$

Fax: $\quad 33-1-45-24-85-00$

U.S. OECD Mission

19 rue Franqueville

75016 Paris, France

DOE Representative

Jean Claude Paye

Robert A. Cornell

Pierre Vinde

M. Taniguchi

Tel:

Fax: $\quad 33-1-45-24-74-80$

Peter Paul Jodoin

33-1-45-24-74-24 


\section{OECD/NEA}

OECD Nuclear Energy Agency

le Seine Saint Germain

12 , boulevard des Isles

Tel: $\quad 33-1-45-24-11-12$

92130 Issy-les-Moulineaux, France

Fax: $\quad 33-1-45-24-11-10$

Director General

Kunihiko Uematsu

Deputy Director General

33-1-45-24-10-00

Samuel Thompson

33-1-45-24-10-02

Deputy Dir., Safety/Regulation

33-1-45-24-10-04

Radiation Protection/Waste Mgmt. Jean-Pierre Olivier

$33-1-45-24-10-40$

Nuclear Safety

Giani Frescura

$33-1-45-24-10-50$

Deputy Dir., Science/Technology

Philippe Savelli

33-1-45-24-10-06

NEA Data Bank

Nuclear Development

Nigel Tubbs

Geoffrey Stevens

33-1-45-24-10-60

\section{MEMBER STATES}

$\begin{array}{llll}\text { Australia } & \text { Austria } & \text { Belgium } & \text { Canada } \\ \text { Denmark } & \text { Finland } & \text { France } & \text { Germany } \\ \text { Greece } & \text { Iceland } & \text { Ireland } & \text { Italy } \\ \text { Japan } & \text { Luxembourg } & \text { Netherlands } & \text { Norway } \\ \text { Portugal } & \text { S. Korea } & \text { Spain } & \text { Sweden } \\ \text { Switzerland } & \text { Turkey } & \text { U.K. } & \text { U.S. }\end{array}$

(contd next page) 


\section{OECD/NEA (contd)}

\section{FUNCTION}

Promote orderly development of peaceful uses of nuclear energy through cooperation among Member States. Initiate, encourage, and coordinate cooperative work in reactor and nuclear fuel cycle studies, radiation protection and waste management, nuclear safety, regulatory matters, and nuclear data collection.

\section{ACTIVITIES}

- Workshops, technical meetings, symposia, and publications

- Joint R\&D programs

- Data Bank

\section{U.S. PARTICIPATION IN WASTE MANAGEMENT ACTIVITIES}

\section{- Radioactive Waste Management Committee (RWMC) -}

Established in 1975; composed of senior experts and government representatives from Member Countries; responsible for national policy, regulation, and program development/implementation; information exchange and discussion forum on waste management policy, regulatory, technical and scientific issues; participation of $\mathrm{CEC}$ and IAEA.

- Performance Assessment Advisory Group (PAAG) - Initiated in 1985 to provide a broad forum for discussion of performance assessment and to advise the RWMC on technical aspects of system performance assessments.

- Coordinating Group on Sile Evaluation and Design of Experiments for Radioactive Waste Disposal (SEDE) Established in 1990, forum for discussions of site characterization issues and promotion of specific studies in this area.

(contd next page) 


\section{OECD/NEA (contd)}

- Probabilistic System Assessment Group (PSAG) - Initiated in 1985; provides a broad forum for discussion and development of probabilistic safety assessment codes; sponsors code intercomparison exercises; reports to the RWMC on the technical aspects of such codes.

\section{- Liaison Commitiee for Cooperative Program on}

Decommissioning

Participants: Belgium, Canada, France, Germany, Italy, Japan, Spain, Sweden, U.K., U.S.

Term: 1990-1995 (Phase 2).

Scope: Exchange of scientific and technical information concerning nuclear installation decommissioning projects.

- Committec on Radiation Protection and Public Health (CRPPH)

- Coordinated Research and Environmentel Survellance Programme (CRESP) - Related to sea disposal of radioactive waste.

Participants: Belgium, Canada, Denmark, France, FRG, Italy, Japan, Netherlands, Portugal, Spain, Sweden, Switzerland, U.K., U.S., IAEA; IMO is an associate member.

Term: 1981-1995.

Scope: Investigate oceanographic and biological characteristics of the northeast Atlantic disposal site and perform related scientific work; as of 1987 , extended to cover land-based discharges.

- Commitlee for Tech./Econ. Studies on Nuclear Enerry Development and Fuel Cycle (NDC)

- Assess, review, and evaluate technical and economic implications related to the nuclear fuel cycle.

Participants: Open to NEA members, IEA, LAEA, CEC. Term: 10-26-77- unspecified.

Scope: Provide governments and scientific communities with competent and reliable information, based on a very wide field of expertise and matured in international debate, to assist in policy discussions. 


\section{PARTIAL NEA ORGANIZATION}

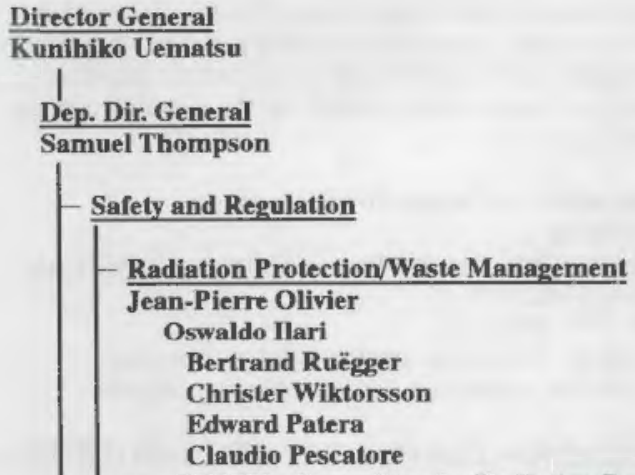

- CRPPH - Committee for Radiation Protection/ Public Health

- RWMC - Radioactive Waste Management Committee

- Nuclear Safety

Giani Frescura Jacques Royen

- CSNI - Committee for Safety of Nuclear Installations

- CNRA - Committee for Nuclear Regulatory Activities

\section{Nuclear Development}

Geoffrey Stevens

James Joosten

Pierre Girouard

- NDC - Committee for Technical/Economic Studies on Nuclear Energy Development/Fuel Cycle

\section{Science and Information Processing}

Philippe Savelli

Nigel Tubbs

- NSC - Nuclear Science Committee 


\section{NUCLEAR SOCIETIES}

\section{AUSTRALIA}

Australian Nuclear Association (ANA) P.O. Bax 445

Sutherland, N.S.W. 2232

Tel: $\quad 61-2-528-8529$

Australia

Fax: $\quad 61-2-543-9263$

\section{BELGIUM}

Forum Nucléaire Belge (ASBL)

Avenue Lloyd George 7

1050 Brussels, Belgium

Tel: $\quad 32-2-647-22-92$

Fax: $\quad 32-2-647-04-54$

Belgian Nuclear Society (BNS)

Ravensteinstreet 3

Tel: $\quad 32-2-774-0511$

1000 Brussels, Belgium

Tel: $\quad 32-2-774-0547$

CANADA

Canadian Nuclear Association (CNA)

144 Front St. West, Suile 725

Toronto, Ontario M5J 2L 7

Tel: $\quad$ 416-977-6152

Canada

Fax: $\quad 416-979-8356$

Canadian Nuclear Society (CNS)

144 Front St. West, Suite 725

Torouto, Ontario M5J 2L7

Tel: $\quad 416-977-7620$

Cauada

Fax: $\quad 416-979-8356$

\section{CHINA}

Chinese Nuclear Society (CNS)

P.O. Box 2125

Beijing 100822, China/PR

Tel: $\quad 86-1-801-2211$

Fax: 86-1-867-188 


\section{CIS}

Nuclear Society International

Kurchatov Square

123182 Moscow

Russian Federation

Tel: $\quad 7-95-196-2073$

Fax: $\quad$ 7.95-943-0074

World Association of Nuclear

Operators (WANO)

c/o State Institute for Nuclear

Power Plant Operation

Fergankaya 25

109507 Moscow

Russian Federation

Tel: $\quad$ 7-95-377-0104

Fax: $\quad 7-95-376-0897$

EUROPE

European Nuclear Society (ENS)

P.O. $\mathrm{Bax} 5032$

Tel: $\quad 41-31-320-6111$

3001 Bern, Switzerland

Fax: $\quad 41-31-382-4466$

Forum Atomique Europeén (FORATOM)

22 Buckingham Gate

London SW1E 6LB

Tel: $\quad 44-71-828-0116$

United Kingdom

Fax: $\quad 44-71-931-0646$

FINLAND

Finnish Nuclear Society (ATS)

Loennrotinkatu 37

Tel: $\quad 358-0-648-931$

00180 Helsinki 18, Finland

Fax: $\quad 358-0-603-626$ 


\section{FRANCE}

Forum Atomique Français 48, rue de la Procession 75015 Paris, France

Tel: $\quad 33-1-45-67-07-70$

Fax: $\quad 33-1-40-65-92-29$

Section Française de l'ANS (SFANS)

c/o Framatome

Tour Fiat, Cedex 16

92084 Paris la Défense

Tel: $\quad 33-1-47-96-14-14$

France

Fax: $\quad 33-1-47-96-30-31$

Societé Française d'Energie

Nucléaire (SFEN)

48, rue de la Procession

75015 Paris, France

Tel: $\quad 33-1-44-49-60-00$

Fax: $\quad 33-1-44-49-60-11$

World Association of Nuclear

Operators (WANO)

39, Avenue de Friedland

Tel: $\quad 33-1-40-42-30-78$

75008 Paris, France

Fax: $\quad 33-1-40-42-92-77$

\section{GERMANY}

Deutsches Atomforum e.V. (DAtF)

Heussallee 10

53113 Bonn 1, Germany

Tel: $\quad 49-228-507-0$

Fax: $\quad 49-228-507-219$

Kerntechnische Gesellschaft e.V.

(KTG)

Heussallex 10

53113 Bonn 1, Germany

Tel: $\quad 49-228-50-7259$

Fax: $\quad 49-228-50-7219$ 
ANS Sezione Locale Italiana c/o Ansaldo S.p.A.

C.so Perrone 25

Tel: $\quad 39-10-655-8505$

16161 Genoa, Italy

Fax: $\quad 39-10-655-8816$

Forum Italiano dell'Energia

Nucleare (FIEN)

Palazzo Taverna

Via di Monte Giordano, 36

00186 Rome, Italy

Tel: $\quad 39-6-689-3091$

Fax: $\quad 39-6-8528-2591$

Società Nucleare Italiana (SNI)

Facolta di Ingegneria

Viale Risorgimento 2

Tel: $\quad 39-51-644-3400$

40136 Bologna, Italy

Fax: $\quad 39-51-644-3411$

\section{JAPAN}

Atomic Energy Society of Japan (AESI)

1-1-13, Shimbashi

Minato-ku, Tokyo 105, Japan

Tel: $\quad 81-3-508-1261$

Fax: $\quad 81-3-581-6128$

Japan Atomic Industrial

Formm (JAIF)

6th Floor, Toshin Bldg.

1-13, Shimbashi

Tel: $\quad$ 81-3-508-2411

Minato-ku, Tokyo 105, Japan

Fax: $\quad 81-3-508-2094$

World Association of Nuclear

Operators (WANO)

c/o Komae Institute, CRIEPI

2-11-1 Iwato-Kita

Komae-shi, Tokyo, Japan

Tel: $\quad 81-3-480-4809$

Fax: $\quad 81-3-480-5379$ 


\section{KOREA}

Korea Atomic Industrial

Forum, Inc. (KAIF)

Yeoeuido P.O. Box 1021

Tel: $\quad 82-2-785-2570$

Seoul 150-610, Korea

Fax: $\quad 82-2-785-3975$

Korean Nuclear Society (KNS)

No. 21, Yeoeuido-dong

Youngdungpo-Ku Box 109

Tel: $\quad 82-2-786-5975$

Seoul 150-610, Korea

Fax: $\quad$ 82-2-786-5975

\section{NETHERLANDS}

Nederlands Atoomforum

P.O. $\operatorname{Box} 1$

1775 ZG Petten

Nethertands

Tel: $\quad 31-2246-4082$

Fax: $\quad 31-2246-3490$

Netherlands Nuclear Society

c/o N.V. Kema

Utrechtsweg 310

6812 AR Arnhem

Tel: $\quad 31-85-56-2491$

Netherlands

Fax: $\quad 31-85-45-8279$

SPAIN

Forum Atómico Español

Boix y Morer, 6

Tel: $\quad 34-12-553-63-03$

28003 Madrid, Spain

Fax: $\quad 34-12-535-08-82$

Sociedad Nuclear Española (SNE)

Campoamor 17

Tel: $\quad 34-1-308-6318$

28004 Madrid, Spain

Fax: 34-1-308-6344 
SWEDEN

Swedish Atomic Forum (SAFO)

Box 1704

11187 Stockholm, Sweden

Tel: $\quad 46-8-790-04-95$

Fax: $\quad 46 \cdot 8-10-78-28$

Föreningen Kärnteknik (FK)

Box 1419

Tel: $\quad 46-8-613-81-46$

11184 Stockholm, Sweden

Fax: $\quad 46-8-796-71-02$

\section{SWITZERLAND}

Schweizerische Vereinigung für

Atomenergie (SVA)

Postfach 5032

3001 Bern, Switzerland

Tel: $\quad 4-31-22-58-82$

Fax: 4-31-22-92-03

Schweizerische Gesellschaft der

Kernfachleute (SGK)

c/o Paul Scherrer Institute

5232 Villigen-PSI

Tel: $\quad 41-56-99-2692$

Switzerland

Fax: $\quad 41-56-98-2327$

\section{UNITED KINGDOM}

British Nuclear Energy Society

(BNES)

1-7 Great George Street

London SW1P 3AA

Tel: $\quad 44-71-222-7722$

United Kingdom

Fax: $\quad 44-71-222-7500$

British Nuclear Forum (BNF)

22 Buckingham Gate

London SW1E 6LB

Tel: $\quad$ 44-71-828-0116

United Kingdom

Fax: $\quad 44-71-828-0110$

(contd next page) 


\section{UNITED KINGDOM (contd)}

Institution of Nuclear Engineers

(INucE)

Allan House

1 Penerley Road, Catford

London SE6 2LQ

Tel: $\quad 44-81-698-1500$

United Kingdom

Fax: $\quad 44-81-695-6409$

World Association of Nuclear

Operators (WANO)

Chelsea Chambers

262a Fulham Rd.

London SW10 9EL

Tel: $\quad 44-71-352-3617$

United Kingdom

Fax: $\quad 44-71-351-9678$

\section{UNITED STATES}

American Nuclear Society (ANS)

555 North Kensington Avenue

Tel: $\quad 312-708-6611$

La Grange Park, IL 60525

Fax: $\quad 312-708-0499$

Nuclear Energy Institute

Suite 400, 1776 I Street NW

Washington, DC 20006-2495

Tel: $\quad 202-739-8000$

Fax: $\quad 202-785-4019$

World Association of Nuclear

Operators (WANO)

Suite 1500

1100 Circle 75 Parkway

Atlanta, GA 30339-3064

Tel: $\quad 404-953-7602$

Fax: 404-953-7549

\section{YUGOSI_AVIA}

Professional Section of ETAN

for Nuclear Technique and

Technology (ETAN-NDE)

c/o Institut Jozef Stefan

Jamova 39

61000 Ljubljana, Yugoslavia

Tel: $\quad 38-61-371-321$

Fax: 38-61-219-365 


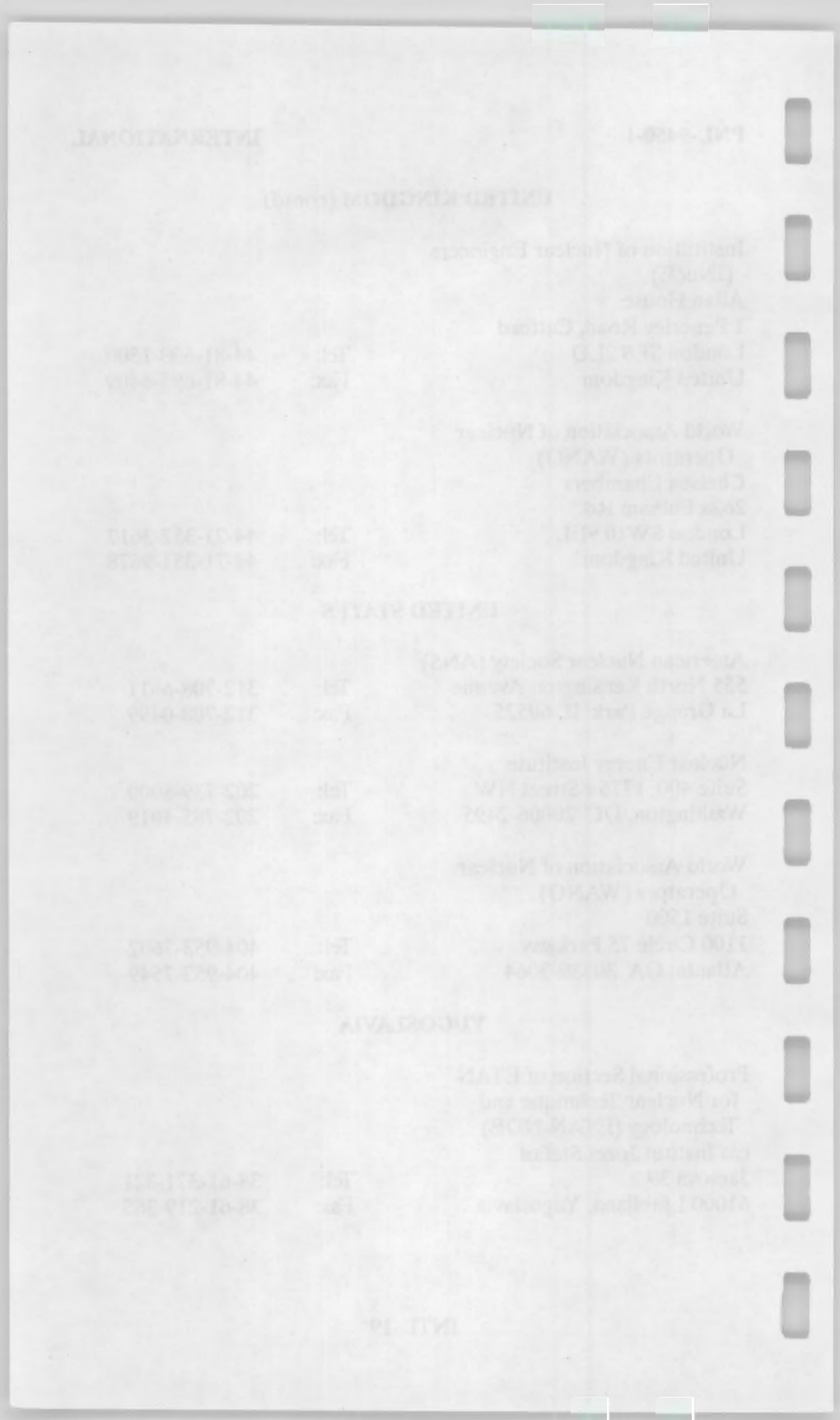




\title{
GLOSSARY
}

\author{
ORGANIZATIONS, FACILITIES \\ TECHNICAL AND OTHER TERMS
}




\section{ORGANIZATIONS AND FACILITIES}

\section{Page}

A

ABÜS

Waste treatment plant

GE-21

ADA

Acid digestion plant

SZ-5

AEA

AEB

$\mathrm{AEC}$

Atomic Energy Authority .......... UK-4

Atomic Energy Bureau...$\ldots \ldots \ldots \ldots$ JA

Atomic Energy Commission $\ldots \ldots \ldots$ IN-6

JA-4

KS-3

SF-2

AEC

Atomic Energy Commission

TW-2

AEC

AEC

AECB

AECL

AEO

AERB

AESJ

AGIP

ANA

ANDRA

ANL

ANPA

ANRE

ANS

ANSTO

ANU

APM

Äspö

ASBL

Asse

(now DOE)

US-14

Atomic Energy Corporation ........ SF-2

Atomic Energy Council ............ TW-2

Atomic Energy Control Board ....... CA-3

Aromic Energy of Canada Limited ... CA-2

Atomic Energy Office ............ KS-3

Atomic Energy Regulation Board .... IN-6

Atomic Energy Society of Japan ...... INTL-16

Nuclear fuel company . .......... IT-4

Australien Nuclear Association ...... INTL-13

Agence Nationale pour la Gestion

des Déchets Radioactifs ......... . CA-2

........ FR-4

Argonne National Laboratory ....... US-6

National Agency for Environmental

Protection ................. IT-2

Agency of Natural Resources \&

Energy ..................... JA-15

American Nuclear Society .......... INTL-15

Australian Nuclear Science and

Technology Organization ......... AS-2

Australian National University . ...... . AS-4

Demonstration Reprocessing plant ... F FR-6

Hard rock laboratory ............. SW-7

Forum Nucléaire Belge . ........... INTL-13

Salt dome repository..$\ldots \ldots \ldots \ldots \ldots$ GE-3 
ATLAS

ATS

AVH

AVM

AWE

B

B205

BAM

BARC

$\mathrm{BCO}$

BEATE

Bel Nipi Energoprom

Reprocessing facility $\ldots \ldots \ldots \ldots \ldots$.

Bundesanstalt für Materialforschung und -prüfung $\ldots \ldots \ldots \ldots \ldots \ldots$.

Bhabha Atomic Research Centre ....

Battelle - Columbus Operations .......

Reprocessing facility

National Research/Design Institute ...

Basic Energy studies ................

BES

BEW

BfS

BGR

BGS

BITF

BINE

BLM

BMFT

BMU

BMWi

BNES

BNF

BNFL

BNL

BNS

BOM

BOR

BP
Bundesamt für Energiewirtschaft ....SZ-3

Bundesamt für Strahlenschutz ...... GE-5

Bundesanstalt für Geowissenschaften und Rohstoffe ................ GE-4

British Geological Survey ........... UK-4

Borehole Instrumentation Test Facility CA-8

Beijing Institute of Nuclear Engineering

$\mathrm{CH}-2$

Bureau of Land Management ........ US-33

Bundesministerium für Forschung und Technologie .............. GE-3

Bundesministerium für Umwelt,

Naturschutz Reaktorsicherheit .... GE-5

Ministry for Economic Affairs ....... GE-8

British Nuclear Energy Society ....... INTL-18

British Nuclear Forum .............. INTL-18

British Nuclear Fuels plc ........... UK-14

Brookhaven National Laboratory .... US

Belgian Nuclear Society ............ INTL-13

Bureau of Mines ................. US-33

Bureau of Reclamation ............. US-33

Belgoprocess ................... BE-4 
BRE

BRGM

(1)

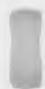

CANMET

Casaccia

CDTN

CEA

CEC

CECE

Cédra

CEN-CA

CEN-FaR

CEN-G

CEN-S

CEN/SCK

CEN-VRH

CHALMERS

CIAE

CIEMAT

Cisra

CIPE

CIS

CLAB
Building Research Establishment .... Bureau de Recherches Géologiques et Minières ..........................

UK-5

FR-8

CA-4

IT-2

ENEA nuclear research center .......

Centro de Desenvolvimento de

Tecnologia Nuclear de Nuclebras ..

Commissariat à l'Énergie Atomique ..

Commission of the European

Communities ................. INTL-1

Combined Electrolysis Catalytic

Exchange System

US-20

Société cooperative nationale pour l'entreposage de déchets radioactifs $\ldots \ldots \ldots \ldots \ldots \ldots \ldots, \quad S Z-4$

Centre d'Études Nucléaires de Cadarache

BR-3

FR-3

Centre d'Études Nucléaires de Fontenay-aux-Roses ...........

Centre d'Études Nucléaires de Grenoble

FR-10

FR-11

Centre d'Études Nucléaires de Saclay . FR-11

Studiecentrum voor Kernenergie/

Centre d'Études de l'Énergie

Nucléaire

BE-7

Centre d'Études Nucléaires

de la Vallée du Rhône

FR-11

SW-4

Technical University ...............

China Institute of Atomic Energy ....

Centro de Investigaciones Energéticas, Medio Ambientales y Tecnologicas .

$\mathrm{CH}-2$

SP-3

Società cooperativa nazionale per

l'immagazzinamento di scorie

radioattive

SZ-4

Interministerial Committee for

Economic Planning ..............

Commonwealth of Independent States INTL-14

Central storage for spent fuel ....... SW-7 
CNA

CNEA

CNEIC

CNEN

CNNC

CNRA

CNS

CNS

CNS

CNS

COGEMA

COMMOX

COMURHEX

COVRA

CPF

CRESP

CRIEPI

CRL

CRPPH

CSN

CSNI

CTC

D

DAE

DAM

DAtF
Canadian Nuclear Association . . . . . . . INTL-13

Comision Nacional de Energia

Atomica .................... AR-3

Chinese Nuclear Energy Industry

Corporation ................. CH-3

Comissão Nacional de Energia

Nnclear $\ldots \ldots \ldots \ldots \ldots \ldots \ldots \ldots$ BR-3

China National Nuclear

Corporation................. $\mathrm{CH}-2$

Committee for Nuclear Regulatory

Activities .................... INTL-12

Canadian Nuclear Society ........... INTL-13

Chinese Nuclear Society ........... INTL-13

Conncil for Nnclear Safety ......... SF-2

Center for Nuclear Studies .......... PK-4

Compagnie Generale des

Matières Nucléaires ............ FR-3

COGEMA subsidiary .............. FR-4

Uranium conversion company ....... FR-4

Centrale Organisatie Voor

Radioactief Afval .............. NL-2

Chemical Processing Facility ........ JA-21

Coordinated Research and Environmental Surveillance Programme (NEA) .................... INTL-11

Central Research Institute of

Electric Power Industry .......... JA

Chalk River Laboratories ........... CA-5

Committee on Radiation Protection and Pnblic Health (NEA) ......... INTL-11

Consejo de Seguridad Nuclear ....... SP-3

Committee for Safety of Nnclear

Installations ................... INTL-12

Computer Training Center ......... PK-4

Department of Atomic Energy ....... IN-6

Direction des Applications

Militaires ..................... FR-5

Deutsches Atomforum ........... INTL-15 
DBE

DD\&PM

DEN

DOA

DOE

DoE

DOI

DOT

Drigg

DWK.

DWPF

E

EARP

EBR-II

EC

ECN

EdF

EdF

EM

ENEA

ENEL

ENI

ENRESA

ENS

ENSM

ENUSA

EP-1, 2

EPA

EPB

EPRI

ERAM
Deutsche Geselischaft zum Bau und Betrieb von Endlagem für Abfallstoffe mbH ............ GE-5

Design Department/Pilot Manufacture BO-3

Department of Energy . . . . . . . . . . UK

Department of Agriculture ......... US-33

Department of Energy . .......... US-2

Department of the Environment .... UK-3

Department of Interior . . . . . . . . US -5

Department of Transportation . . . . . . U US-5

Waste disposal facility $\ldots \ldots \ldots \ldots \ldots$ UK-6

Deutsche Gesellschaft für Wiederaufarbeitung von Kembrennstoffen ... GE-6

Defense Waste Processing Facility .... US-24

Enhanced Actinide Removal Plant ... UK-14

Experimental Breeder Reactor No. 2 . US $=14$

European Community ............ INTL-2

Stichting Energieonderzoek

Centrum Nederland ............ NL-2

Electricité de France ........... FR-A

Engineering Demonstration Facility .. JA-21

DOE Environmental Management ... US US

Ente per le Nuove Technologie,

l'Energia l'Ambiente .......... IT-2

Ente Nazionale per l'Energia Elettrica IT-2

Ente Nazionale Idrocarburi ........ . IT-2

Empresa Nacional de Residuos

Radioactivos .............. SP-4

European Nuclear Society . . . . . . . . . . INTL-14

Ecole Nationale Supérieure des

Mines de Paris ............... FR-16

Empresa Nacional del Uranio ...... . SP-4

Waste treatment facilities .......... . UK-14

Environmental Protection Agency , ... U. US-5

Electric Power Bureau ............. KS-3

Electric Power Research Institute . . . . US-29

Endlager für Radioaktive

Abfälle Morsleben ............. GE-5 
ERS

ESKOM

ETAN-NDE

ETF

EUREX

Eurobitum

EURODIF

Eurowatt

Ezeiza

F

FBFC

FIEN

FK

FN

FORATOM

FRAGEMA

FRG

FURNAS

FUSRAP

G

GHZ GIRIO

GNS

Gorleben

Gosatomnadzor

Goscomchemobyl

Goscomecologija
Effluent Recovery System .......... US-20

South African utility .............. SF-2

Professional Section of ETAN for Nuclear

Technique and Technology ........ INTL-19

Engineering Test Facility ............ JA-21

Fuel reprocessing pilot plant ........ IT-3

Bituminization plant $\ldots \ldots \ldots \ldots \ldots \ldots$ BE-4

Commercial enrichment company .... FR-2

Solvent treatment hot pilot plant ..... B BE-4

Argentine atomic center .......... AR-2

Société Franco-Belge de Fabrication de Combustibles (Belgium and .... BE -5 France) $\ldots \ldots \ldots \ldots \ldots \ldots \ldots \ldots, \quad F R-4$ Forum Italiano dell'Energia

Nucleare .................... INTL-16

Föreningen Kärnteknik ............. INTL-18

Fabricazioni Nucleari $. . . \ldots \ldots \ldots . . \quad$ IT-2

Forum Atomique Européen . ........ INTL-14

COGEMA subsidiary...$\ldots \ldots \ldots \ldots$ FR-4

Federal Republic of Germany ....... GE-3

Subsidiary of Electrobrás .......... BR-3

Renedial action program $\ldots \ldots \ldots \ldots$ US-14

Hot cell facility $\ldots \ldots \ldots \ldots \ldots \ldots \ldots$ GE-16

Government Industrial

Research Institute ............ . JA-8

Gesellschaft für Nuklear-Service ..... GE-6

Repository site ................ GE-2

Russian Federal Authority for

Nuclear Radiation/Safety ........ RS-3

State Chernobyl Committee ........ BO-2

State Committee on Environment

Protection ................... BO-2

G-6 


\section{PNL-9450-1}

Gospromatomnadzor

State Committee on Supervision of Industrial and Nuclear Safety ...... BO B 2

GRS

GSC

GSF/IT

Gesellschaft für Reaktorsicherheit ... GE-13

Geological Survey of Canada ....... CA-4

Forschungszentrum für Umwelt und Gesundheit $\ldots \ldots \ldots \ldots \ldots \ldots$ GE-4

GSP POLESJE State Specialized Enterprise/

State Chernobyl Committee ....... BO-2

H

HADES

HFEF

HISS

HITACHI

HMIP

$\mathrm{HQ}$

HSE

HSK

Underground Research Laboratory ..

BE-8

Hot Fuel Examination Facility ....... US-14

Hydrogen Isotope Separation

System .................. US -20

Hitachi, Ltd . . . . . . . . . . . . IA-8

H.M. Inspectorate of Pollution . . . . . . UK-4

DOE-Headquarters ............. US-4

Health and Safety Executive ....... UK-5

Nuclear Safety Inspectorate ....... SZ-3

I

IAEA International Atomic Energy

ICRP

Agency $\ldots \ldots \ldots \ldots \ldots \ldots \ldots \ldots$ INTL -4

International Commission on

Radiological Protection ......... INTL-8

ICT

IEN

IFTF

IGCAR

IHI

Institute of Chemical Technology ..... GE-16

Instituto de Engenharia Nuclear ..... BR-3

Immobilized Fuel Test Facility ...... . CA-9

Indira Ghandi Centre for Atomic

Research ............... IN-7

Ishikawajima-Harima Heavy

Industries $\ldots \ldots \ldots \ldots \ldots \ldots \ldots \ldots$ JA-9

IMO International Maritime Organization . INTL-11

INB Industrias Nncleares do Brasil ...... BR-3

INE Institute for Nnclear Waste

Technology ............... GE-17

INEL Idaho National Engineering

Laboratory ................ US-6 
INER

INET

INucE

IOS

IPEN

IPEP

IPSN

IRD

IREP

IRUS

IRW

ISF

IVO

$J$

IAERI

JAIF

JET

JGC

JNFL

JPDR

JRC

K

KAERI

KAIF

KAIST

KALPAKKAM KANUPP
Institute of Nuclear Energy

Research .................. TW-2

Institute of Nuclear Energy

Technology .................. $\mathrm{CH}-2$

Institntion of Nnclear Engineers ..... INTL-19

Institnte of Oceanographic Sciences ... UK-4

Institnto de Pesquisas Energeticas

e Nucleares .................. BR-3

Institute of Power Engineering Problems BO-1

CEA-Institut de Protection et de Sureté

Nucléaire ................. FR-5

Instituto de Radioproteçâo e

Dosimetria .................. BR-3

Institute of Radio-Ecological Problems $\quad \mathrm{BO}-3$

Intrusion Resistant Underground

Structnre ................. CA-7

Institnte of Reactor Materials ....... GE-16

Interim Storage Facility ............ IN-8

Imatran Voima $\mathrm{Oy} . . . \ldots \ldots \ldots \ldots$ FI-1

Japan Atomic Energy Research

Institnte .................. IA-4

Japan Atomic Industrial Forum ..... . . INTL-16

Joint Enropean Torus ............. UK-9

JGC Corporation ................ JA-12

Japan Nuclear Fnel Ltd. ........... JA-4

Japan Power Demonstration

Reactor $\ldots \ldots \ldots \ldots \ldots \ldots \ldots \ldots$ JA-6

Joint Research Center (CEC) . ...... INTL-3

Korea Atomic Energy Research

Institute $\ldots \ldots \ldots \ldots \ldots \ldots \ldots \ldots, \quad$ KS -3

Korea Atomic Industrial Forum ...... INTL-17

Korea Advanced Institute of

Science/Technology ............. KS-3

Fuel reprocessing laboratory ......... IN-2

Karachi Nuclear Power Plant ....... PK-3 


KEMA
KEMAKTA
KEPCO
KEWA
KFA
KFK
Khlopin
KIER

KINS

KNFC

KNS

KOBE

KOLAR

Konrad

KOPEC

KPA-STORE

KRF

KS-KT-100

KTG

KTH

Kurchatov

$L$

LAube

La Manche

LANL

LBRMF

LLNL
LA HAGUE

N.V. Tot Keuring van Electro-

technische Materialen Arnhem .... NL-5

Kemakta Konsult ............... SW-4

Korea Electric Power Corporation ... KS-3

Kernbrennstoff Wiederaufarbeitungstechnik $\mathrm{GmbH} \ldots \ldots \ldots \ldots$ GE-6

Kernforschungszentrum Jülich ....... GE-4

Kernforschungszentrum Karlsruhe ... GE-4

Khlopin Radium Institute .......... RS-3

Korea Institute of Energy and Resources .................. KS-3

Korea Institute of Nuclear Safety Technology .................. KS-3

Korea Nuclear Fuel Co. .......... . KS-3

Korean Nuclear Society ............ INTL-17

Kobe Steel, Ltd. ................. JA-14

Waste disposal research station ...... IN-7

(Iron mine) repository site ......... GE-2

Korea Power Engineering Co. ...... KS-3

Spent nuclear fuel storage facility .... FI $\quad$ FI-6

Krypton recovery pilot plant ......... JA-21

Cold pilot vitrification plant ........ RS-10

Kerntechnische Gesellschaft ........ INTL-15

Royal Institute of Technology ........ SW-4

Russian Kurchatov Institute ......... RS-3

Disposal Facility ................ FR-7

COGEMA, Centre de la Hague ...... FR-2

Disposal Facility ................ FR-7

Los A Amos National Laboratory ..... . US-6

Large' Block Radionuclide Migration

Facility ................... CA 10

Lawrence Livermore National

Laboratory .................. US-6

M

$\mathrm{M} \& \mathrm{O}$

MAFF

Management and Operating

Contractor ................... US-26

Ministry of Agriculture, Fisheries

and Food

UK-4 
MAPI

MAPS

Mayak

MET

MINATOM

MITI

MMC

MOD

MOFA

MONJU

MOST

MRS

$N$

Nagra

NCS

NDC

NEA

NEC

NERC

NERSA

NFC

NFF

NII

NIREX

NIRAS

NIRS

NMSS

NMU

NNSA

NPO
Ministry of Atomic Power and Industry RS-2

Madras Atomic Power Station ....... IN-8

Production Association ............ R. RS-7

Mineral/Energy Technology ......... CA-4

Ministry for Atomic Energy of the Russian Federation ........... RS-3

Ministry of International Trade \& Industry .................. JA-4

Mitsubishi Metal Corporation ....... JA-15

Ministry of Defense ............. UK-5

Ministry of Foreign Affairs ......... JA-16

Japanese LMFBR .............. JA-3

Ministry of Science and Technology ... KS-2

Monitored Retrievable Storage ...... US-2

Nationale Genossenschaft für die

Lagerung Radioaktiver Abfälle .... SZ SZ-2

Nuclear Cargo Service ............. GE-6

NEA Technical/Economic Studies .... INTL-11

Nuclear Energy Agency (OECD) .... INTL-9

National Energy Council ........... SF-2

National Environment Research Council .................. UK-5

Groupement Centrale Nncléaire

Européene à Neutrons Rapides .... F FR-4

Nnclear Fnel Complex ............. IN-8

CH-4

Nuclear Fuel Fabrication ............ CH-5

Nuclear Installations Inspectorate .... UK-4

UK Nirex Ltd. . .................. UK-4

Nationale Instelling voor Radioactief Afval en Splijtstoffen ............ BE-3

National Institute of Radiological

Sciences ...................... JA-4

Nuclear Material Safety and

Safeguards .................. US-7

Niedersächsisches Umweltministerinm GE-5

National Nuclear Safety

Administration .............. $\mathrm{CH}-2$

Waste Management Organization .... KS-3 
NRC

NRPB

NRR

NSB

NSC

NUCLECO

NUKEM

NUMATEC

NWCF

NWPA

NWPAA

NWTRB

0

OCRWM

OECD

OH

OIP

ONDRAF

ONWN

OPLA

ORNL

P

PAAG

PAEC

PAMELA

PARR-1, 2

Pelindaba

PEV
Nuclear Regulatory Commission .....

US-1

National Radiological Protection

Board .................... UK-4

NRC Office of Nuclear Reactor

Regulation ............... US-7

National Safety Bureau ........... JA-4

Nuclear Safety Commission ....... JA-4

Waste Management Services company IT-2

Nuclear fuel services company ...... GE G

COGEMA Inc. subsidiary .......... FR-13

New Waste Calcining Facility ....... US-8

Nuclear Waste Policy Act $\ldots \ldots \ldots \ldots$ US-2

Nuclear Waste Policy Amendments

Act $\ldots \ldots \ldots \ldots \ldots \ldots \ldots \ldots \ldots$ US -2

Nuclear Waste Technical

Review Board ................

US-32

US-20

Waste Management ...........

Organisation for Economic Coopera-

tion and Development . . . . . . . INTL-8

Ontario Hydro ..............

NRC Office of International Programs US-7

Organisme National de Déchets

Radioactifs et des Matières

Fissiles ................. BE-2

Office of the U.S. Nuclear Waste

Negotiator ................ US -32

National research program ......... NL-2

Oak Ridge National Laboratory ...... U US-6

Performance Assessment Advisory

Group .................. INTL-10

Pakistan Atomic Energy Commission . PK-2

Vitrification pilot plant ......... GE-18

Research/Training reactors ......... PK-4

National Nuclear Research Center ... SF-2

Prototype vitrification facility ....... FR-12 
PFR

Phenix

PINSTECH

PIVER

PIVER II

PKA

PKS

PNC

PNL

PREFRE

PSAG

PSI

PWSF

PWTF

R

RADWASS

RES

RF

RFP

RIVM

RLWTF

RMDF

RPA

RSK

RW

RWMAC

RWMC
Reprocessing plant

UK-2

French FBR $\ldots \ldots \ldots \ldots \ldots \ldots \ldots$ FR-4

Pakistan Institute of Science/

Technology ................. PK-2

Hot pilot plant - vitrification ......... FR-12

HLW vitrification facility ........... FR-12

Pilot fuel conditioning plant ......... GE-6

Quality assurance project $\ldots \ldots \ldots \ldots$ GE-16

Power Reactor and Nuclear Fuel

Development Corporation ........ JA-3

Pacific Northwest Laboratory ........ U. US-6

Fuel reprocessing plant ............ IN-9

Probabilistic System Assessment

Group (NEA) . . . . . . . . . . INTL-11

Paul Scherrer Institute $. . . \ldots \ldots \ldots . \quad$ SZ-3

Pu-contaminated Waste Storage

Facility .................... JA-22

Pu-contaminated Waste Trealment

Facility...$\ldots \ldots \ldots \ldots \ldots \ldots$ JA-22

Radioactive Waste Safety Standards .. INTL-4

NRC Office of Nuclear Regulatory

Research .................... US-7

Russian Federation ............... RS-3

Rocky Flats Plant .............. US 6

Rijksinstituut voor Volksgezondheid

en Milieuhygiene ............... NL-6

Radioactive Liquid Waste Treatment

Facility ..................... US 14

Radioactive Materials Disposal

Facility ..................... US-16

Research Production Association .... RS-3

Reaktor Sicherheitskommission ...... GE $\quad$ GE-5

DOE-Office of Civilian Radioactive

Waste Management ............. US-4

Rad. Waste Management Advisory

Committee ................. UK-5

Radioactive Waste Management

Center ..................... 
PNL-9450-1

RWMC

RWMC

RWOS

$S$

SAE

SAFO

Saluggia

SBH

SCK/CEN

SEDE

SFANS

SFEN

SFMP

SFR

SGAB

SGK

SGN

SICN

SKB

SKI

SNE

SNI

SNI

SNL

SPD

SRS

SSI

SSK

SSSF

STA
Rad. Waste Management

Committee (NEA) ............ INTL-10

Radioactive Waste Management

Complex ................. US-17

Radioactive Waste Operations Site ... CA-12

Strategic Business Secretariat ........ BR-3

Swedish Atomic Forum . ........... INTL-18

ENEA nuclear research center ....... IT-2

Siemens Brennelementewerk Hanau .. GE-6

Studiecentrum voor Kernenergie/

Centre d'Études de l'Énergie

Nucléaire ................. BE-7

Site Evaluation and Design of Experi-

ments for Radioactive Waste

Disposal (NEA) ............... INTL-10

Section Française d'Energie Nucléaire INTL-15

Societé Française d'Energie Nucléaire INTL-15

Surplus Facilities Management

Program ...................... US-14

Swedish Final Repository .......... SW-7

Sveriges Geologiska .............. SW-5

Schweizerische Gesellschaft der Kernfachleute .............. INTL-18

Société Générale pour les Techniques

Nouvelles .................. FR-4

COGEMA subsidiary ............. FR-4

Svensk Kärnbränslehantering ....... SW-3

Statens Kärnkraftinspektion ......... SW-3

Sociedad Nuclear Española . ......... INTL-17

Belgian utility $\ldots \ldots \ldots \ldots \ldots \ldots \ldots$ BE-8

Società Nucleare Italiana . . . . . . . . . . INTL-16

Sandia National Laboratories ........ US

Sodium Process Demonstration

Facility .................... US 14

Savannah River Site ............. US-24

Statens Straalskyddsinstitut .......... SW-3

Strahlenschutzkommission .......... GE-5

Solid Storage Surveillance Facility .... IN IN-10

Science and Technology Agency ...... JA 


\section{STE3 \\ STEM \\ STMI \\ STUDSVIK STUK}

SuperPhenix SVA

SWA

SWEPP

SYNATOM

$T$

TAIPOWER

TAN

TAPS

TAWRS

TECHNI-

CATOME

THORP

TN

TREAT

Trisaia

Trombay

TRUEX

TRW

TUM

TVF

TVO

TWRS

$U$

UKAEA

UNVIE

UP1
Liquid waste treatment facility ...... FR-15

Simulation Test Facility for Environmental Radionuclide Migration ... JA-11

Nuclear services company .......... F FR-4

Studsvik Energiteknik ............ SW-8

Finnish Center for Radiation and

Nuclear Safety ................. FI-2

European FBR ................ FR-1

Schweizerische Vereinigung für Atomenergie ................ INTL-18

Waste water treatment plant ......... GE-21

Stored Waste Examination Pilot

Plant ...................... US-17

Belgian company .............. BE-3

Taiwan Power Company $\ldots \ldots \ldots \ldots \ldots$.
Test Area North $\ldots \ldots \ldots \ldots \ldots \ldots \ldots$

Tarapur Atomic Power Station ....... IN-9

Tritium Aqueous Waste Recovery

System

US-20

Nuclear fuel cycle services company .. $\quad$ FR-4

Thermal Oxide Reprocessing Plant ... U UK-2

Transnucléaire .................... FR-18

Transient Reactor Test Facility ....... U. US-14

ENEA nuclear fuel services company .................. IT-2

Fuel reprocessing plant ............. IN-2

TRU waste technology .............. US-14

TRW Environmental Safety Systems .. . US-27

Technische Universität München ..... GE-6

Tokai Vitrification Facility .......... JA-22

Teollisuuden Voima Oy ............ F1-1

Tank Waste Remediation System ..... US-27

UK Atomic Energy Authority ....... UK-3 U.S. Mission to IAEA . . . . . . . . . . INTL-6 Fuel reprocessing plant $\ldots \ldots \ldots \ldots \ldots$ FR-14 
UP2

UP2-800

UP3

URENCO

URL

USGS

USSI

V

Vaalputs

Valindaba

VKTA

VLJ

VNIPIET

VTT

Fuel reprocessing plant $\ldots \ldots \ldots \ldots \ldots$.

FR-3

Fuel reprocessing plant $\ldots \ldots \ldots \ldots \ldots$ FR-3

Fuel reprocessing plant $\ldots \ldots \ldots \ldots \ldots$ FR-3

Uranium enrichment consortium ..... NL-1

Underground Research Laboratory .. $\quad$ CA-8

U.S. Geological Survey ............ US-5

COGEMA subsidiary ..........., FR-4

LLW disposal facility ............ SF-2

U enrichment and conversion plants .. $\quad$ SF-2

Verein für Kernferfahrenstechnik and

Analytik Rossendorf ............ GE-21

LLW/ILW repository ............. FI-6

All Russian Design/Research Association RS-3 Technical Research Centre of

Finland $\ldots \ldots \ldots \ldots \ldots \ldots \ldots \ldots, \quad$ FI-2

\section{W}

WAK

WAMAP

WANO

WASTEF

WATRP

WDF

WEAF

WEC

WERF

WHC

WINCO

WIP

WIPP

WL

WSRC

WTC

Wiederaufarbeitungsanlage

Karlsruhe ................... GE-6

Waste Management Advisory

Program .................... INTL-5

World Association of Nuclear

Operators ................... INTL-14

Glove box and hot cell facilities ...... JA-11

International peer review program .... INTL-5

Waste Dismantling Facility ......... JA-19

Waste Examination Assay Facility .... US-20

Westinghouse Electric Company ..... US-10

Waste Environmental Reduction

Facility ..................... US 17

Westinghouse Hanford Company .... US-6

Westinghouse Idaho National

Company .................. US-6

Waste Immobilization Plant ........ IN-5

Waste Isolation Pilot Plant ......... US-3

Whilshell Laboratories ............ CA-5

Westinghouse Savannah River Co. ... U US-6

Waste Treatment Center ............ CA-7 
PNL-9450-1

WTC

WVNS

WVRF
Waste Treatment Complex ......... UK-14

West Valley Nuclear Services ........ US-6

Waste Volume Reduction Facility .... CA-12

Y

YMP

Yucca Mountain Project

US-33

YMPO

YMSCP

Yucca Mountain Project Office ...... US-6

Yucca Mountain Site Characterization

Project .................... US 19

$Z$

ZFK-DE

ZPPR

Waste treatment project ........... GE-16

ZWILAG

Zero Power Plutonium Reactor ....... US-14

Zwischenlager Würenlingen AG ..... SZ S 
TECHNICAL AND OTHER TERMS

(A)

/a

Acad

AS

AFR

AGR

AR

ATR

BWR

CAD

CAM

CANDU

CEO

CIP

COB

$\mathrm{COO}$

CTC

$/ \mathrm{d}$

DAM

DAS

D\&D

DOG

FBR

FBTR

FRP

GCHWR

GCR

GSP

GWd

GWe

h

HAO

HAWC
Acting

per annum

Academian

Assistant Secretary

Away-from-reactor

Advanced gas-cooled reactor

At-reactor

Advanced thermal reactor

Boiling water reactor

Computer aided design

Computer aided manufacturing

Canadian deuterium uranium reactor

Chief Executive Officer

Cold isostatic pressing

Chairman of the Board

Chief Operating Officer

Computer training center

per day

Deputy Assistant Manager

Deputy Assistant Secretary

Decontamination and decommissioning

Dissolver off-gas

Fast breeder reactor

Fast breeder test reactor

Fuel reprocessing plant

Gas-cooled, heavy water moderated reactor

Gas-cooled, graphite moderated reactor

Gel-supported precipitation

Gigawatt day

$10^{9}$ watts of electricity ( $\left.1000 \mathrm{MWe}\right)$

per hour

Head-end oxide

High acid waste content 
HEPA

HLLW

HLW

HIP

HTGR

HTR

HWLWR

HWR

ILW

$\mathrm{kg} / \mathrm{h}$

kgHM

$\mathrm{kgU}$

kPa

$\mathrm{kW}$

1h

LEU

LGR

LHGW

LLLW

LLW

LTR

LMFBR

LWCHW

LWR

m

MEV

MLW

MOX

MTR

MTIHM

MTU

MW

MWd/t

MWe

MWt
High-efficiency particulate absolute

High-level liquid waste

High-level waste

Hot isostatic pressing

High-temperature, gas-cooled reactor

High-temperature reactor

Heavy water moderated, light water cooled

reactor (same as LWCHW)

Heavy-water reactor

Intermediate-level waste

kilograms per hour

kilograms heavy metal

kilograms uranium

kilopascal

kilowatt

liters per hour

Low enriched uranium

Light-water cooled, graphite moderated reactor

Low heat generating waste

Low-level liquid waste

Low-level waste

Low-temperature district heating reactor

Liquid metal fast breeder reactor

Light-water-cooled heavy-water-moderated

reactor (same as HWLWR)

Light water reactor

meter

Million electron volts

Medium-level waste (same as intermediate-level)

Mixed (plutonium/uranium) oxide

Materials test reactor

Metric tons initial heavy metal

Mega tons uranium

Megawatts

Megawatt days per ton

Megawatts electric

Megawatts thermal 


\section{PNL-9450-1}

NPT

OTD

PFR

PHWR

PLWR

PM

Pu

PUREX

PWR

QUAD

$R \& D$

SBR

SF

SS

SWU

SYNROC

1

TD

Th/U

tHM

THTR

TPO

TRU

tU

TWh

$\mathrm{U}$

$\mathrm{UF}_{6}$

$\mathrm{UO}_{2}$

VOG
Non-Proliferation Treaty

Office of Technical Development

Prototype fast reactor

Pressurized heavy water reactor

Pressurized light water reactor

Program manager

Plutonium

$\mathrm{Pu} / \mathrm{U}$ redox extraction process

Pressurized water reactor

$10^{15} \mathrm{Btu}$

Research and development

Fast breeder reactor (European acronym)

Spent fuel

Stainless steel

Separative work (U enrichment)

Synthetic rock (for waste immobilization)

Metric ton

Technical development

Thorium/uranium

Metric tons heavy metal

Thorium high-temperature reactor

Technical program officer

Transuranic

Metric tons uranium

Terawatt hour (million megawatt hours)

Uranjum

Uranium hexaflouride

Uranium dioxide

Vessel off-gas 


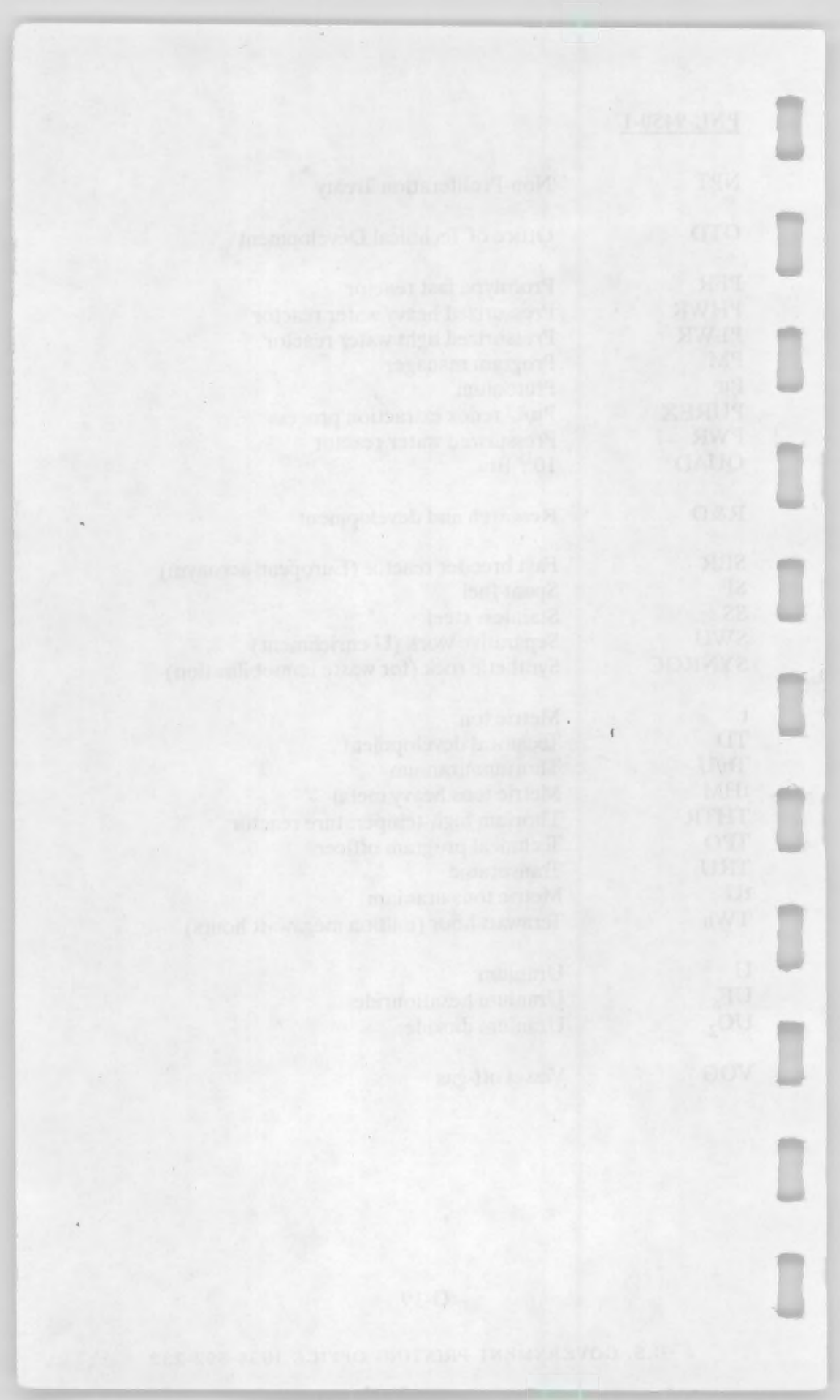

